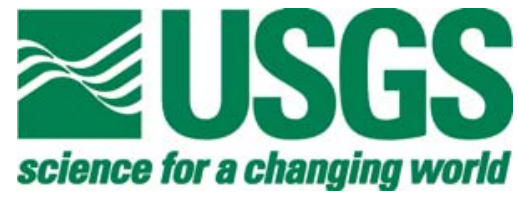

Prepared in cooperation with the North Carolina Geological Survey and the University of North Carolina, Wilmington

\title{
Preliminary Physical Stratigraphy and Geophysical Data of the USGS Hope Plantation Core (BE-110), Bertie County, North Carolina
}

By Robert E. Weems, Ellen L. Seefelt, Beth M. Wrege, Jean M. Self-Trail, David C. Prowell, Colleen Durand, Eugene F. Cobbs III, and Kevin C. McKinney

Any use of trade, product, or firm names is for descriptive purposes only and does not imply endorsement by the U.S. Government.

Open-File Report 2007-1251

U.S. Department of the Interior

U.S. Geological Survey 


\section{DIRK KEMPTHORNE, Secretary \\ U.S. Geological Survey \\ Mark D. Myers, Director}

U.S. Geological Survey, Reston, Virginia 2007

Revised and reprinted: 2007

For product and ordering information:

World Wide Web: http://www.usgs.gov/pubprod

Telephone: 1-888-ASK-USGS

For more information on the USGS - the Federal source for science about the Earth, its natural and living resources, natural hazards, and the environment:

World Wide Web: http://www.usgs.gov

Telephone: 1-888-ASK-USGS

Suggested citation:

Weems, R.E., Seefelt, E.L., Wrege, B.M., Self-Trail, J.M., Prowell, D.C., Durand, C., Cobbs III, E.F., and McKinney, K.C., 2007, Preliminary physical stratigraphy and geophysical data of the USGS Hope Plantation core (BE-110), Bertie County, North Carolina: U.S. Geological Survey Open-File Report 2007-1251.

Any use of trade, product, or firm names is for descriptive purposes only and does not imply endorsement by the U.S. Government.

Although this report is in the public domain, permission must be secured from the individual copyright owners to reproduce any copyrighted material contained within this report. 


\section{CONTENTS}

Introduction

Physical Stratigraphy and Lithology

Hydrogeology and Geophysical Logging

Acknowledgments

References

FIGURES

1. Map of eastern North Carolina showing the location of Hope Plantation in Bertie County.

2. Topographic map and areal photograph showing the location of corehole BE-110-2004.

3. Monitoring well construction report.

4. Plot showing the correlation between lithology and geophysical data in BE-110-2004.

\section{PHOTOGRAPHS}

\begin{tabular}{|c|c|c|c|c|}
\hline Box 1 & Box 21 & Box 41 & Box 61 & Box 81 \\
\hline Box 2 & Box 22 & Box 42 & Box 62 & Box 82 \\
\hline Box 3 & Box 23 & Box 43 & Box 63 & Box 83 \\
\hline Box 4 & Box 24 & Box 44 & Box 64 & Box 84 \\
\hline Box 5 & Box 25 & Box 45 & Box 65 & Box 85 \\
\hline Box 6 & Box 26 & Box 46 & Box 66 & Box 86 \\
\hline Box 7 & Box 27 & Box 47 & Box 67 & Box 87 \\
\hline Box 8 & Box 28 & Box 48 & Box 68 & Box 88 \\
\hline Box 9 & Box 29 & Box 49 & Box 69 & Box 89 \\
\hline Box 10 & Box 30 & Box 50 & Box 70 & Box 90 \\
\hline Box 11 & Box 31 & Box 51 & Box 71 & Box 91 \\
\hline Box 12 & Box 32 & Box 52 & Box 72 & Box 92 \\
\hline Box 13 & Box 33 & Box 53 & Box 73 & Box 93 \\
\hline Box 14 & Box 34 & Box 54 & Box 74 & Box 94 \\
\hline Box 15 & Box 35 & Box 55 & Box 75 & Box 95 \\
\hline Box 16 & Box 36 & Box 56 & Box 76 & Box 96 \\
\hline Box 17 & Box 37 & Box 57 & Box 77 & Box 97 \\
\hline Box 18 & Box 38 & Box 58 & Box 78 & Box 98 \\
\hline Box 19 & Box 39 & Box 59 & Box 79 & Box 99 \\
\hline Box 20 & Box 40 & Box 60 & Box 80 & \\
\hline
\end{tabular}




\section{TABLES}

Table 1. Nannofossil occurrence chart.

\section{APPENDICES}

Appendix 1. Hope Plantation lithologic log Appendix 2. Hope Plantation run log

Appendix 3. Hope Plantation sampling log 


\section{Introduction}

In March and April, 2004, the U.S. Geological Survey (USGS), in cooperation with the North Carolina Geological Survey (NCGS) and the Raleigh Water Resources Discipline (WRD), drilled a stratigraphic test hole and well in Bertie County, North Carolina (fig. 1). The Hope Plantation test hole (BE-110-2004) was cored on the property of Hope Plantation near Windsor, North Carolina. The drill site is located on the Republican 7.5 minute quadradrangle at lat $36^{\circ} 01^{\prime} 58^{\prime \prime}$ N., long $78^{\circ} 01^{\prime} 09^{\prime \prime} \mathrm{W}$. (decimal degrees 36.0329 and 77.0192) (fig. 2). The altitude of the site is $48 \mathrm{ft}$ above mean sea level as determined by Paulin Precise altimeter. This test hole was continuously cored by Eugene F. Cobbs, III and Kevin C. McKinney (USGS) to a total depth of $1094.5 \mathrm{ft}$. Later, a ground water observation well was installed with a screened interval between 315-329 feet below land surface (fig. 3). Upper Triassic, Lower Cretaceous, Upper Cretaceous, Tertiary, and Quaternary sediments were recovered from the site. The core is stored at the NCGS Coastal Plain core storage facility in Raleigh, North Carolina.

In this report, we provide the initial lithostratigraphic summary recorded at the drill site along with site core photographs, data from the geophysical logger, calcareous nannofossil biostratigraphic correlations (Table 1) and initial hydrogeologic interpretations. The lithostratigraphy from this core can be compared to previous investigations of the Elizabethtown corehole, near Elizabethtown, North Carolina in Bladen County (Self-Trail, Wrege, and others, 2004), the Kure Beach corehole, near Wilmington, North Carolina in New Hanover County (Self-Trail, Prowell, and Christopher, 2004), the Esso \#1, Esso \#2, Mobil \#1 and Mobil \#2 cores in the Albermarle and Pamlico Sounds (Zarra, 1989), and the Cape Fear River outcrops in Bladen County (Farrell, 1998; Farrell and others, 2001). This core is the third in a series of planned benchmark coreholes that will be used to elucidate the physical stratigraphy, facies, thickness, and hydrogeology of the Tertiary and Cretaceous Coastal Plain sediments of North Carolina.

\section{Physical stratigraphy and lithology}

General lithologic descriptions were made at the drill site. These descriptions are summarized briefly here and in detail within Appendix 1. Sediment colors are based on The Geological Society of America Rock Color Chart (Goddard and others, 1995) and all colors are taken from wet samples. Stratigraphic nomenclature follows Gohn (1992), as modified by Prowell and others (2003) Self-Trail, Prowell, and Christopher (2004), and Sohl and Owens (1991). In the past, Prowell and Self-Trail among others generally have described as "silty clay" the lithology here designated as "very clayey silt." In this report, however, we do not claim the presence of such an extremely fine lithology because we do not yet have definitive grain-size analyses to confirm the presence of true clay beds in this core.

Corehole BE-110-2004 penetrated 1094.5 feet of Triassic, Cretaceous, Tertiary, and Quaternary sediments (fig. 4). The Triassic rocks, which have been slightly metamorphosed thermally, are assigned to the Carnian-Norian Chatham Group of the Newark Supergroup (Weems and Olsen, 1997). The Lower Cretaceous section is provisionally assigned to the Aptian Patuxent Formation of the Potomac Group on the basis of its lithology and position at the base of the Coastal Plain section. The lower portion of the Upper Cretaceous section (previously referred in North Carolina to the "Eaglefordian" by Murray, 1961) is provisionally assigned to the Clubhouse Formation (Cenomanian), and an unnamed formation (Turonian) that has been incorrectly called the "Cape Fear Formation" in the deep subsurface of South Carolina (Gohn and others, 1977; Hazel and others, 1977; Hattner and Wise, 1980; Christopher, 1982; Fallaw and others, 1990; Gohn and Campbell, 1991; Frederickson and others, 1991; Gohn, 1992; Martin and Simones, 1992; Falls and others, 1993; Gellici and Logan, 
1993; Radosevich and Klein, 1993; Snipes and others, 1993; Martin and Simones, 1994; Martin and others, 1994; Poppe and others, 1994; Fallaw and Price, 1995; Howe and others, 1996; Falls and others, 1997a,b; Temples and Engelhardt, 1997; Watson and others, 1997; Christopher and others, 1999; Christopher and others, 2000; Falls and Prowell, 2001; Frederiksen and others, 2001; Gellici and others, 2001). This unnamed formation is overlain by the upper Coniacianlower Santonian Cape Fear Formation interbedded with tongues of the laterally equivalent Pleasant Creek Formation. Above this is the lower portion of the middle Santonianupper Campanian Tar Heel Formation of the Black Creek Group, and an unnamed upper Campanian formation that lies stratigraphically above the Donoho Creek Formation of the Black Creek Group and below the Peedee Formation. Field identification of lag deposits and unconformable surfaces suggests that the Tar Heel Formation of the Black Creek Group can be subdivided into units that correlate with the Shepherd Grove and Caddin Formations of South Carolina. The Upper Cretaceous sec-tion is overlain unconformably by the Lower Tertiary (Paleocene, Danian) Beaufort Group (Harris and Laws, 1994), which in turn is overlain unconformably by the Upper Tertiary (upper Pliocene) Yorktown and Chowan River Formations. The youngest unit recovered at this site was the Quaternary Charles City Formation.

Core BE-110 was sampled for microfossil abundance, strontium analysis, and water analysis (fig. 4, Appendix 3). Typically, units thought to represent marginal marine to fully marine settings were sampled for calcareous nannofossils and dinoflagellates, while pollen samples were extracted from marginal marine to nonmarine sediments. Assignment here of lithologic units to particular formations may require revision in light of results from forthcoming paleontological analyses of the samples taken.

\section{Hydrogeology and geophysical logging}

Hydrologic data collected from the Hope Plantation stratigraphic core hole included hydrology-oriented lithologic descriptions, borehole geophysical well logs, and water level. The entire Hope Plantation core was examined on site immediately upon recovery for physical characteristics that affect porosity and permeability. Hydrology oriented lithologic descriptions were derived from core samples following the standards of Wentworth (1922). Borehole geophysical well logs were collected, using standard induction and gamma protocols as described by Keys (1984), to determine the thickness of formations, make aquifer determinations, and to provide information for the design of the observation well. The 1095-ft Hope Plantation core hole was drilled during two separate sessions and geophysically logged after each session. The first round of drilling ran from March 8 to March 27, 2004; the second round ran from April 1 to April 18, 2004. Borehole geophysical surveys were run through the drill rod or in the open borehole after each round of drilling. Geophysical logs were collected on March 12 in the open hole (after the borehole had passed a depth of $40 \mathrm{ft}$ prior to installing surface casing), on March 27 (after the first round of drilling), and on April 18 (after the final round of drilling when the drill had reached basement). The compilation of this suite of logs provides a complete profile of the borehole. A natural gamma log was collected from within the inner barrel using a Century Geophysical Corporation model 8043A resistance tool. The second complete set was collected in an open borehole using a Century model 9511A induction tool. Each hole was logged twice: once in the up and once in the down direction. Copies of the logs are available in a raw LAS format on the USGS website http://nc.water.usgs.gov/ccp/2004Hope/data.html

. Water level was used to determine the head pressure differential of the screened interval. This was done with a piezometer that was installed in a discrete water-bearing zone and used to directly measure water level (Heath, 1980). 
Hydrogeologic units in the North Carolina Coastal Plain are defined as sequences of mappable hydrologic packages of sediment that have similar physical characteristics. These units come in two basic types: aquifers and confining units. In this region, aquifers are composed of saturated or partially saturated sediments or limestones. Because of their physical characteristics (such as porosity, permeability and transmissivity) aquifers yield significant quantities of water to wells. Aquifers may be composed of a geologic formation, a group of geologic formations, or a portion of a formation or formations. An aquifer is not necessarily limited to individual geologic units, which means that it may be composed of interconnected saturated materials of different geologic ages. Except for the surficial aquifer, aquifers by definition occur in strata between adjacent confining units that separate aquifers. In the North Carolina Coastal Plain, a confining unit is primarily composed of material that has low permeability (clay, silt, and clayey sand). The general regional geology and permeability distribution that occurs below the Hope Plantation site has been defined by Brown and others (1972). The lithology and extent of various hydrologic formations that make up the North Carolina Coastal Plain aquifers are described by Winner and Coble (1989) in a RASA (Regional Aquifer Systems Analysis) report of the hydrologic framework of the Carolina Coastal Plain. This hydrogeologic framework was used to define the regional aquifers, describe their anticipated order, and provide initial model parameter estimates for porosity, permeability and transmissivity. The upper four aquifers and three confining units are saturated or partly saturated with freshwater. For purposes of this report, saline water is defined as water with chloride concentrations equal to or greater than $250 \mathrm{mg} / \mathrm{L}$. (U.S. Environmental Protection Agency, 1991).

The Hope Plantation stratigraphic core hole encountered six aquifers: the Surficial, Yorktown, Black Creek, Cape Fear and two deeper aquifers. The Yorktown Aquifer is comprised of undifferentiated Yorktown and
Beaufort aquifers, because in this area there is no confining unit between them. The Upper Cape Fear Aquifer contains four discrete zones of high porosity and permeability, separated by partialconfining units. The deepest interval of this aquifer (315 to 329 feet depth) has potential as another source of potable water for the area. Because of this, an observation well was installed in this lowest zone of the Upper Cape Fear Aquifer. There is no head data, but we surmise that the Lower Cape Fear Aquifer may be separated from the Upper Cape Fear Aquifer at this location. The lowest two aquifers in this well are located within the Patuxent Formation of the Potomac group. In Bertie County, where the Hope Plantation stratigraphic core hole is located, water used for agriculture is generally derived from the surficial and Yorktown aquifers, and residential water supplies usually are extracted from the Black Creek Aquifer. The majority of the municipal and industrial watersupply wells in the surrounding area tap the Cape Fear Aquifer (Brown and others, 1972).

Aquifers deeper than these often contain brackish water and may yield water with fluoride in excess of recommended drinking water standards (Brown, 1959). These deeper formations are generally not used for drinking water. The hydrostratigraphy summarized as follows:

$$
\begin{array}{cc}
\text { Depth (feet) } & \\
0.0-1.0 & \text { top soil } \\
1.0-28.0 & \text { Surficial Aquifer } \\
28.0-91.0 & \text { Yorktown Confining Unit } \\
91.0-95.0 & \text { Yorktown Aquifer } \\
95.0-121.0 & \text { Beaufort Aquifer } \\
121.0-160.0 & \text { Black Creek Confining Unit } \\
160.0-188.4 & \text { Black Creek Aquifer } \\
188.4-208.0 & \text { Upper Cape Fear } \\
208.0-329.0 & \text { Confining Unit } \\
& \text { Apper Cape Fear Aquifer } \\
& \text { B. 2408.0 - 224.0 }-250.0
\end{array}
$$$$
\text { Black Creek Confining Unit }
$$ 

C. $261.0-276.0$
D. $315.0-329.0$

$\begin{array}{cc}329.0-443.0 & \begin{array}{c}\text { Lower Cape Fear } \\ \text { Confining Unit }\end{array} \\ 443.0-472.0 & \text { Lower Cape Fear Aquifer } \\ 472.0-759.0 & \text { Confining Unit } \\ 759.0-847.5 & \text { Aquifer } \\ 847.5-866.0 & \text { Confining Unit } \\ 866.0-891.0 & \text { Aquifer } \\ 891.0-1026.0 & \begin{array}{l}\text { Potomac Group } \\ \text { silts and clays }\end{array} \\ 1026.0-1094.5 & \text { Triassic basin rock }\end{array}$

Johnson Well Drilling was contracted to install a groundwater observation well in the partially sealed borehole drilled by the USGS.

This well was installed in the open 329 -ft surface section. A sandpack was installed in the annular space from $304-324 \mathrm{ft}$ above the natural pack. The well was constructed of solid 2-in schedule40 PVC with a screen interval from $314-324 \mathrm{ft}$ below land surface. A $2 \times 2$-ft concrete pad was installed for well-head protection at the surface, and the top of the casing was capped by a steel well-head cover. The top of casing extends above the pad to a height of $3.5 \mathrm{ft}$ above land surface. Static water level was $52 \mathrm{ft}$ below the top of casing. The well was developed using an airlift method. The well subsequently yielded 45 gallons per minute (gpm), but this was not the maximum potential rate because flow rate was limited by pump size. This information is summarized in Figure 3.

\section{Acknowledgments}

The Hope Plantation corehole was drilled by USGS drillers Eugene F. Cobbs, III and Kevin C. McKinney, using a hydraulic-rotary, wirelinecoring rig. We thank Kathleen Farrell and the North Carolina Geological Survey for providing core-storage facilities. We also thank LuAnn W. Joyner and the rest of the staff and management of Hope Plantation for allowing us access to their property and facilities, and for allowing temporary storage of core and equipment. Reviews by Lucy Edwards (USGS) and W. Burleigh Harris (University of North Carolina at Wilmington) are gratefully acknowledged. 


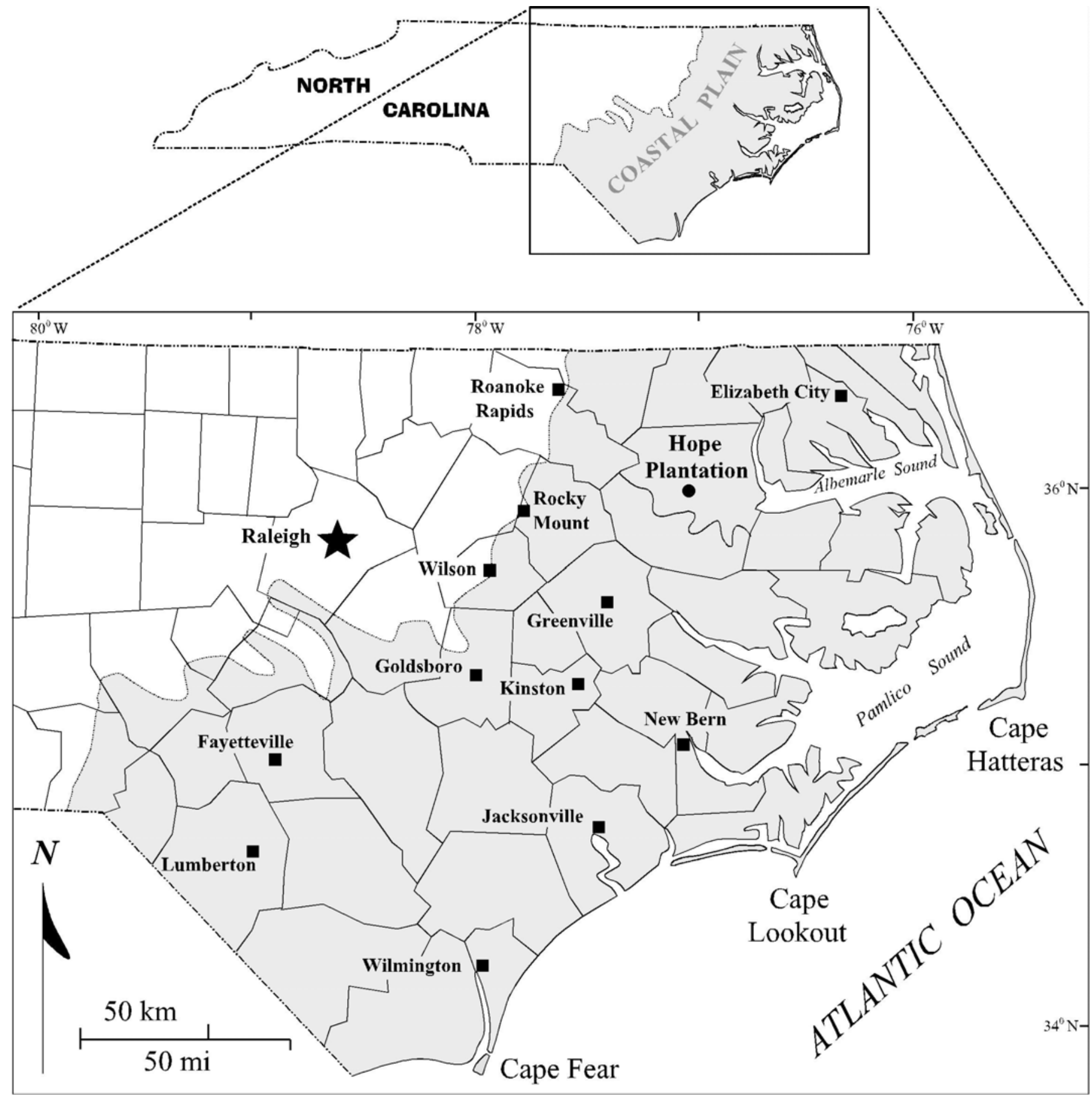

Figure 1 -- Map of eastern North Carolina showing the location of Hope Plantation in Bertie County. 


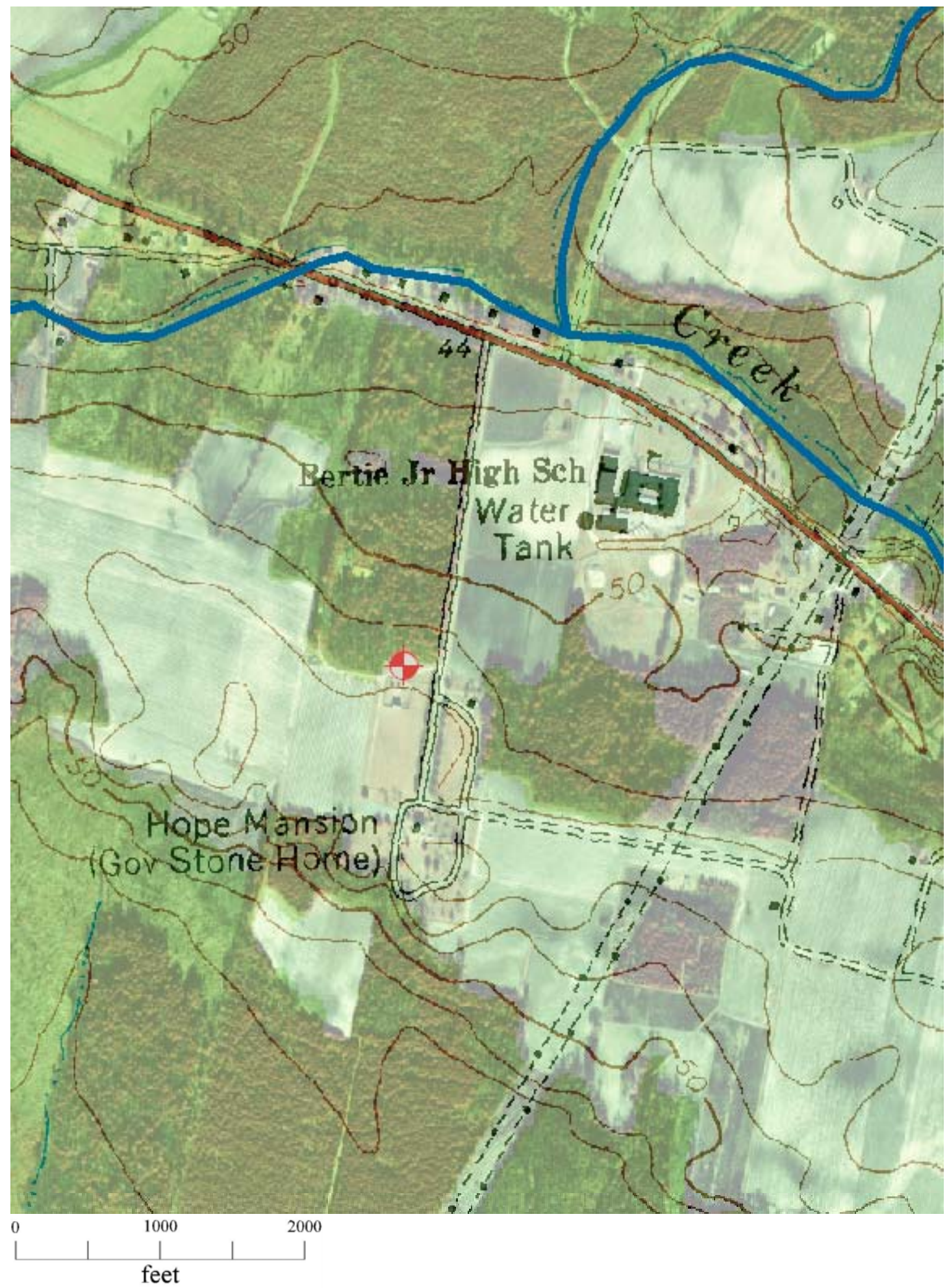

Figure 2. USGS topographic map and areal photograph showing the location of corehole BE-110-2004 $\left(36.0329^{0} \mathrm{~N}, 77.0192^{\circ} \mathrm{W}\right)$ in the Republican 7.5 minute quadrangle, Bertie County, N.C. 


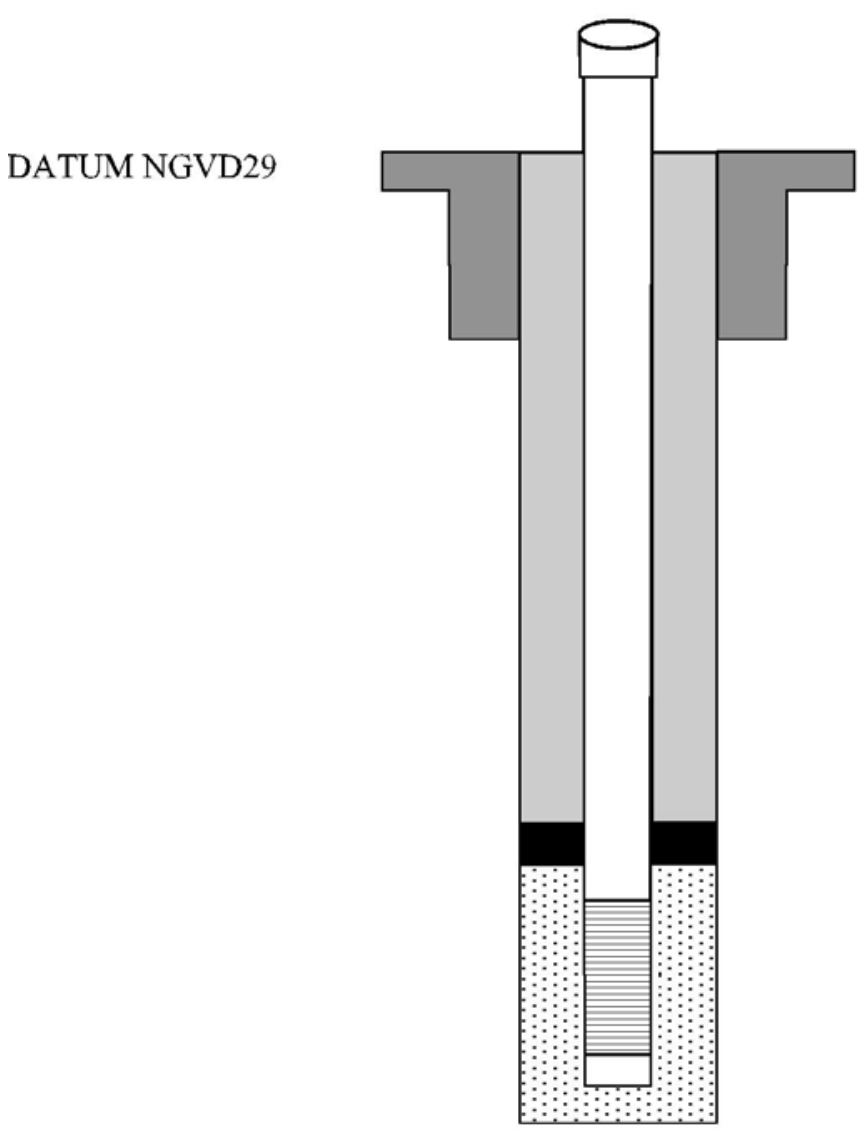

Land Surface Altitude: 48 feet

Altitude of measuring point: 51.5 feet

Height of measuring point: 3.5 feet above land surface

Borehole Diameter: 8 inches

Casing Diameter / Type: 6 inches

Surface Casing Depth: 20 feet

Type of Grout: Type I Portland Cement

Depth of Grout: 0 - 25 feet

Top of Filter Pack Depth: 304 feet

Type of Filter PackSand: \#10 DSI

Screen Slot Number: \#2

Screen Diameter: 2 inch

Screen Interval Top: 314 feet

Screen Interval Bottom: 324 feet

Well Depth: 329 feet

Hole Depth: 1095 feet

Figure 3 - Monitoring Well Construction Report 


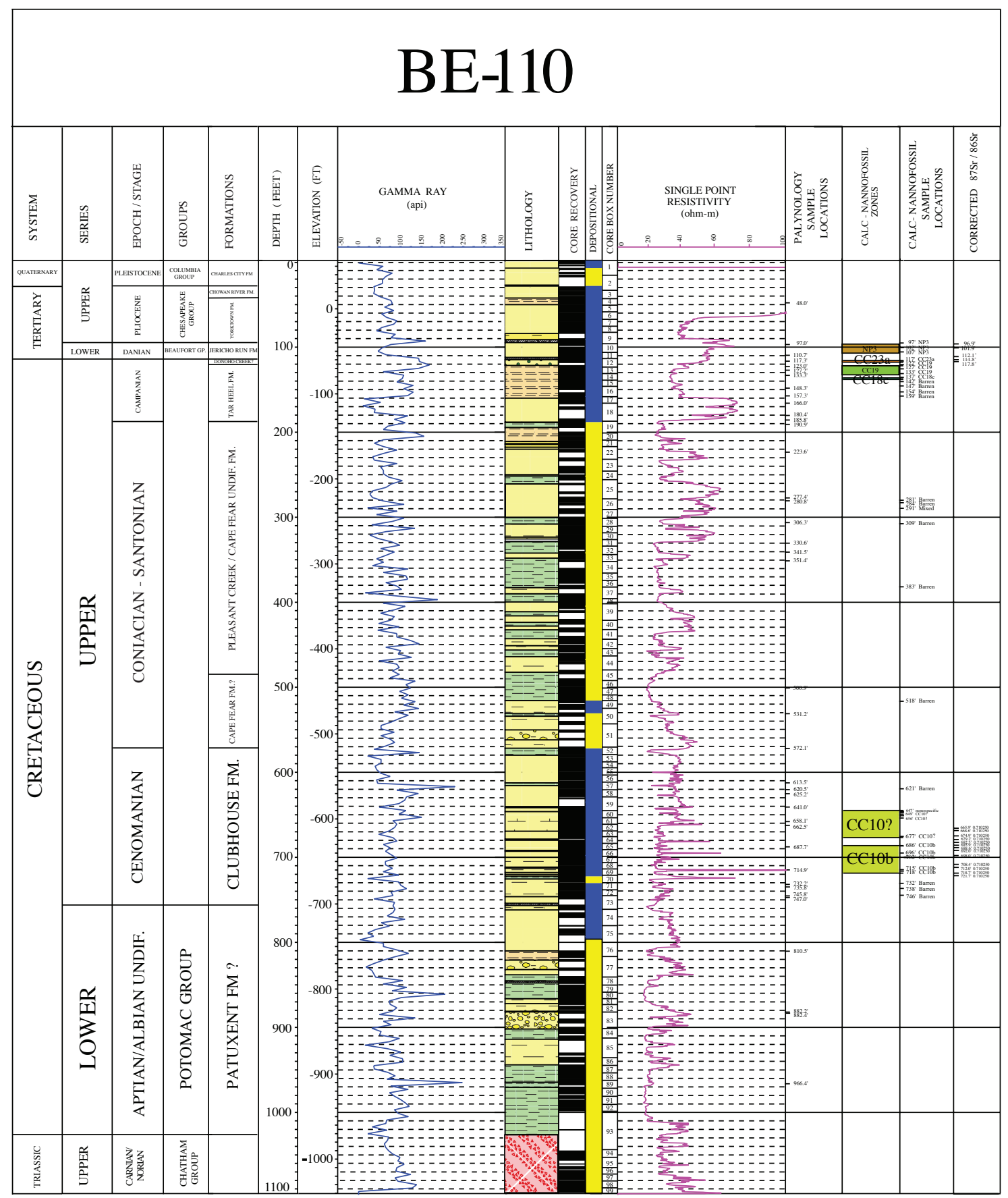

\section{Explanation}

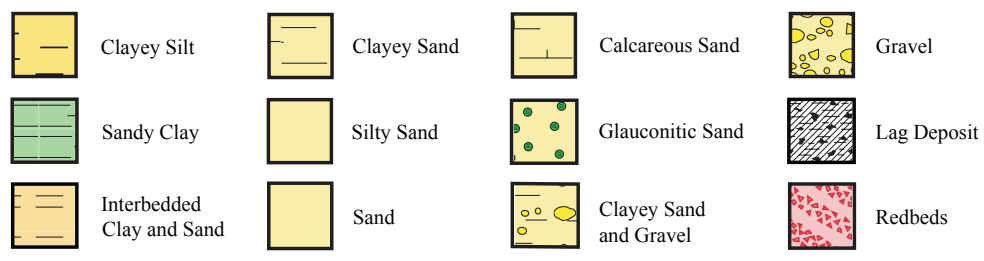

Figure 4. Plot showing the correlation between lithology and geophysical data in BE-110-2004. 


\section{References}

Brown, P.M., 1959, Geology and ground-water resources in the Greenville area, North Carolina: North Carolina Department of Conservation and Development Bulletin 73, 87 p.

Brown, P.M. Miller, J.A., and Swain, F.M., 1972, Structural and stratigraphic framework, and spatial distribution of permeability of the Atlantic Coastal Plain, North Carolina to New York: U.S. Geological Survey Professional Paper 796, 79 p.

Christopher, R.A., 1982, Palynostratigraphy of the basal Cretaceous units of the eastern Gulf and southern Atlantic coastal plains: Georgia Geologic Survey, Information Circular 53, p. 10-23.

Christopher, R.A., Self-Trail, J.M., Prowell, D.C., and Gohn, G.S., 1999, The stratigraphic importance of the Late Cretaceous pollen genus Sohlipollis gen. nov. in the Coastal Plain province: South Carolina Geology, v. 41, p. 27-44.

Christopher, R.A., Prowell, D.C., Self-Trail, J.M., Gohn, G.S., and Waters, K.E., 2000, Problems and inconsistencies in correlating the Middendorf Formation (Upper Cretaceous) throughout the Coastal Plain of South Carolina: Geological Society of America, Abstracts with Programs, v. 32, n. 2, p. 11.

Fallaw, W. C., and Price, Van, 1995, Stratigraphy of the Savannah River Site and vicinity: Southeastern Geology, v. 35, n. 1, p. 21-58.

Fallaw, W. C., Thayer, P.A., and Price, Van, 1990, Basal coastal plain deposits in southwestern South Carolina: Geological Society of America, Abstracts with Programs, v. 22, n. 4, p. 13.

Falls, W., and Prowell, D.C., 2001, Stratigraphy and depositional environments of sediments from five cores from Screven and Burke counties, Georgia: U. S. Geological Survey Professional Paper 1603, p. A1-A20.

Falls, W.F., Baum, J.S., and Prowell, D.C., 1997, Physical stratigraphy and hydrostratigraphy of Upper Cretaceous and Paleocene sediments, Burke and Screven counties, Georgia: Southeastern Geology, v. 36, n. 4, p. 153-176.

Falls, W.F., Baum, J.S., Harrelson, L.G., Brown, L.H., and Jerden, J.L., Jr., 1997, Geology and hydrogeology of Cretaceous and Tertiary strata, and confinement in the vicinity of the U.S. Department of Energy Savannah River Site, South Carolina and Georgia: U. S. Geological Survey, Water-Resources Investigation WRI 97-4245, 125 p.

Falls, W. F., Prowell, D.C., Edwards, L.E., Frederiksen, N.O., Gibson, T.G., Bybell, L.M., and Gohn, G.S., 1993, Preliminary correlation of geologic units in three coreholes along the Savannah River in Burke and Screven counties, Georgia: Geological Society of America, Abstracts with Programs, v. 25, n. 4, p. 14.

Farrell, K.M., 1998, The Cretaceous Cape Fear and Black Creek Formations of Southeastern North Carolina: Cape Fear River traverse-milepost 110 to Milepost 60, in Field Trip Guidebook, 
Carolina Geological Survey Annual Meeting: Durham, Duke University Press, in association with the North Carolina Geological Survey.

Farrell, K.M., Ward, L.W., and Heron, S.D., Jr., 2001, Cape Fear River transect: The Upper Cretaceous Cape Fear, Black Creek, and Peedee Formations of Southeastern North Carolina and the overlying Cenozoic section (Milepost 111 to Milepost 25), Hoffman, C.W., ed., in Field Trip Guidebook, $50^{\text {th }}$ Annual Meeting, Southeastern Section, Geological Society of America, Raleigh, North Carolina, p. 93-118.

Frederiksen, N.O., Edwards, L.E., and Litwin, R.J., 2001, Palynomorph biostratigraphy and paleoecology of Upper Cretaceous sediments from four cores from Screven and Burke counties, Georgia: U. S. Geological Survey Professional Paper 1603, p. C1-C32.

Fredrickson, J.K., Balkwill, D.L., Zachara, J.M., Li, S.W., and Brockman, F.J., 1991, Physiological diversity and distributions of heterotrophic bacteria in deep Cretaceous sediments of the Atlantic Coastal Plain: Applied and Environmental Microbiology, v. 57, n. 1, p. 402-411.

Gellici, J.A., and Logan, W.R., 1993, Preliminary stratigraphic and hydrostratigraphic correlation of four deep coreholes east of the Savannah River Site in Allendale, Barnwell and Aiken counties, South Carolina: Geological Society of America, Abstracts with Programs, v. 25, n. 4, p. 17.

Gellici, J.A., Waters, K.E., and Prowell, D.C., 2001, Hydrostratigraphic units delineated from a deep corehole in Orangeburg, South Carolina: Geological Society of America, Abstracts with Programs, v. 33, n. 2, p. 26.

Goddard, E.N., Trask, P.D., DeFord, R.K., Rove, O.N., Singewald, J.T., Jr., and Overbeck, RM., 1995, The Geological Society of America, Rock Color Chart: Boulder, Colorado.

Gohn, G.S., 1992, Revised nomenclature, definitions, and correlations for the Cretaceous formations in USGS-Clubhouse Crossroads \#1, Dorchester County, South Carolina: U.S. Geological Survey Professional Paper 1518, 39 p.

Gohn, G.S., and Campbell, B.G., 1991, Recent revisions to the stratigraphy of subsurface Cretaceous sediments in the Charleston, South Carolina, area: South Carolina Geology, v. 34, n. 1-2, p. 25-38.

Gohn, G. S., Higgins, B. B., Smith, C. C., and Owens, J. P., 1977, Lithostratigraphy of the deep corehole (Clubhouse Crossroads corehole 1) near Charleston, South Carolina: Studies related to the Charleston, South Carolina, earthquake of 1886; a preliminary report, U. S. Geological Survey Professional Paper 1028, p. 59-70.

Harris, W.B., and Laws, R.A., 1994, Paleogene sediments on the axis of the Cape Fear Arch, Long Bay, North Carolina: Southeastern Geology, v. 34, n. 4, p. 185-199.

Hattner, J.G., and Wise, S.W., Jr, 1980, Upper Cretaceous calcareous nannofossil biostratigraphy of South Carolina: South Carolina Geology, v. 24, n. 2, p. 41-117. 
Hazel, J. E., Bybell, L. M., Christopher, R. A., Frederiksen, N. O., May, F. E., McLean, D. M., Poore, R. Z., Smith, C. C., Sohl, N. F., Valentine, P. C., and Witmer, R. J., 1977, Biostratigraphy of the deep corehole (Clubhouse Crossroads corehole 1) near Charleston, South Carolina: Studies related to the Charleston, South Carolina, earthquake of 1886; a preliminary report, U. S. Geological Survey Professional Paper, p. 71-89.

Heath, R. C., 1980, Basic elements of ground-water hydrology with reference to conditions in North Carolina: United States Geological Survey Water Resources Investigations Open-File Report 8044.

Howe, K. A., Karimjee, A. H., Thibault, J. J., Snipes, D. S., Hodges, R. A., Sherrill, J., and Aadland, R., 1996, Application of Terrastation software to the correlation of Late Cretaceous stratigraphic units at the Savannah River Site, South Carolina: Geological Society of America, Abstracts with Programs, v. 28, n. 2, p. 16.

Keys, W. S., 1984. A synthesis of borehole geophysical data at the underground research laboratory, Manitoba, Canada: Technical Report 15, Battelle Project Management Division Office of Crystalline Repository Development, Columbus, Ohio.

Martin, A. J., and Simones, G. C. , 1992, Applications of ichnology to hydrogeology, with examples from the Cape Fear Formation (Cretaceous), South Carolina: Geological Society of America, Abstracts with Programs, v. 24, n. 7, p. 253-254.

Martin, A. J., and Simones, G. C., 1994, Late Cretaceous ichnofacies, Savannah River Site, Atlantic Coastal Plain, South Carolina; relation to depositional environments and hydrostratigraphy: Geological Society of America, Abstracts with Programs, v. 26, n. 7, p. 411.

Martin, A. J., Close, J. C., and Simones, G. C., 1994, Theoretical and practical considerations of integrating subsurface ichnologic and geophysical data in Late Cretaceous strata, southeastern Atlantic Coastal Plain, USA: International Sedimentological Congress, v. 14, p. S5.2-S5.3.

Murray, G. E., 1961, Geology of the Atlantic and Gulf Coastal Plain province of North America: New York, Harper and Brothers Geoscience Series, 692 p.

Poppe, L. J., Popenoe, P., Poag, C. W., and Swift, B. A. , 1994, Stratigraphy and paleoenvironments of the Southeast Georgia Embayment derived from exploratory wells: Geological Society of America, Abstracts with Programs, v. 26, n. 7, p. 248.

Prowell, D. C., Christopher, R. A., Waters, K. E., and Nix, S. K., 2003, The chrono- and lithostratigraphic significance of the type section of the Middendorf Formation, Chesterfield County, South Carolina: Southeastern Geology, v. 42, n. 1, p. 47-66.

Radosevich, M., and Klein, D. A., 1993, Bacterial enumeration and mercury volatilization in deep subsurface sediment samples: Bulletin of Environmental Contamination and Toxicology, v. 51, n. 2, p. 226-233. 
Self-Trail, J. M., Prowell, D. C., and Christopher, R. A., 2004, The Collins Creek and Pleasant Creek Formations: two new subsurface units in the Atlantic Coastal Plain: Southeastern Geology, v. 42, n. 4., p. 237-252.

Self-Trail, J. M., Wrege, B. M., Prowell, D. C., Seefelt, E. L., and Weems, R. E., 2004, Preliminary Physical Stratigraphy and Geophysical Data of the USGS Elizabethtown Core (BL-244/BL-C-12003), Bladen County, North Carolina: U.S. Geological Survey Open-File Report 2004-1301, 26 p.

Snipes, D. S., Warner, R. D., Price, Van, Jr, and Thayer, P. A., 1993, Metamorphism of Triassic sediments from the Dunbarton Basin, South Carolina: Geological Society of America, Abstracts with Programs, v. 25, n. 4, p. 70-71.

Sohl, N. F., and Owens, J. P., 1991, Cretaceous stratigraphy of the Carolina coastal plain, in Horton, J.W., Jr., and Zullo, V.A., eds., The geology of the Carolinas: Carolina Geological Society, 50th Anniversary Volume, p. 191-220.

Temples, T.J., and Engelhardt, D.W., 1997, Stratigraphy, depositional environments and sequence stratigraphy of the Upper Cretaceous in the Hilton Head Island Test Well Beaufort County, South Carolina: Southeastern Geology, v. 36, n. 4, p. 177-186.

U.S. Environmental Protection Agency, 1991, Secondary maximum contaminant levels (section 143.3 of part 143, National secondary drinking water regulations): U.S. Code of Federal Regulations, Title 40, Parts 100 to 149, revised through July 1, 1991.

Watson, S. P., Sharp, B., Snipes, D. S., and Aadland, R. K., 1997, The Appleton confining system; an unique aquitard in the central Savannah River area, South Carolina: Geological Society of America, Abstracts with Programs, v. 29, n. 3, p. 77.

Weems, R. E., and Olsen, P. E., 1997, Synthesis and revision of groups within the Newark Supergroup, Eastern North America: Bulletin of the Geological Society of America, v. 109, n. 2, p. 195-209.

Wentworth, C. R., 1922, A scale of grade and class terms for clastic sediments: Journal of Geology, v. 30, p. 377-392.

Winner, M. D., and Coble, R. W. 1989, Hydrogeologic framework of the North Carolina Coastal Plain aquifer system: U.S. Geological Survey Open-File Report 87-690, 155p.

Zarra, L., 1989, Sequence stratigraphy and foraminiferal biostratigraphy for selected wells in the Albemarle Embayment, North Carolina: North Carolina Geological Survey Open File Report 89-5, $48 \mathrm{p}$. 
APPENDIX 1. Hope Plantation lithologic log. Common lithologic abbreviations include: vf (very fine), $\mathrm{f}$ (fine), $\mathrm{m}$ (medium), c (coarse), and vc (very coarse) for sand sizes; $\mathrm{tr}$ (trace $=<1 \%$ ); $\mathrm{HCl}$ (hydrochloric acid). Land surface elevation is 48 feet above mean sea level.

\section{0 - 3.0 ft: Run 1}

\section{Disturbed Earth}

$0.0-0.4 \mathrm{ft}: \quad$ VARIED, sand and clay, swirled, varicolored. Box 1.

\section{Charles City Formation}

$0.4-1.3 \mathrm{ft}: \quad$ SAND, quartz, silty, f-m, subangular; organic-rich, black (N1). Box 1.

$1.3-1.9 \mathrm{ft}$ : SAND, quartz, f-m, subangular; yellow (10YR 7/6) and light brownish gray (10YR 6/2). Box 1.

$1.9-3.0 \mathrm{ft}$ : No recovery.

\section{0 - 7.0 ft: Run 2}

$3.0-3.9 \mathrm{ft}: \quad$ SAND, quartz, f-m grading down to $\mathrm{m}-\mathrm{c}$ then back to $\mathrm{f}-\mathrm{m}$, subround; light olive gray (5Y 6/1) grading down to light bluish gray (5B 7/1). Box 1 .

3.9 - 4.2 ft.: $\quad$ SAND, quartz, vf-f, silty and clayey; light bluish gray (5B 7/1). Box 1 .

$4.2-4.5 \mathrm{ft}$ : $\quad$ SAND, quartz, f, with scattered grains up to c, subangular, silty; dark yellowish orange (10YR 6/6). Box 1.

$4.5-7.0 \mathrm{ft}: \quad$ No recovery.

\section{0 - 12.0 ft: Run 3}

$7.0-7.5 \mathrm{ft}: \quad$ SAND, quartz, m-c, thixotropic; estuarine?; greenish gray $(5 G Y 6 / 1)$ grading down to dark yellowish orange (10YR 6/6). Box 1 .

$7.5-7.7 \mathrm{ft}$ : SAND, quartz, vf-f, silty and clayey; faintly banded, paleosol?; olive gray (5Y 3/2). Box 1.

$7.7-9.0 \mathrm{ft}$ : $\quad$ SAND, quartz, m-vc, very poorly sorted, silty; massive, fluvial?; medium brownish gray $(10 Y R 5 / 1)$ grading near base to medium gray $(N 5)$; very coarse quartz grains bluish. Box 1.

$9.0-12.0 \mathrm{ft}$ : No recovery.

\section{0 - 15.0 ft: Run 4}

$12.0-12.9 \mathrm{ft}: \quad$ SAND, quartz, silty and clayey, f-c, very poorly sorted, subangular; moderate yellowish brown (10YR 5/4) grading down to brownish gray (5YR 4/1). Box 1.

$12.9-13.6 \mathrm{ft}$ : SAND, quartz, f-c, very poorly sorted, subround, clean, thrixotropic; yellowish gray $(5 Y 7 / 2)$ with rare blue quartz grains. Box 1.

$13.6-15.0 \mathrm{ft}$ : No recovery. 


\section{0 - 20.0 ft: Run 5}

$15.0-17.7 \mathrm{ft}: \quad$ SAND, quartz, feldspathic, f-vc, very poorly sorted, subangular; gravelly with quartz clasts up to $1 \mathrm{~cm}$ at top but only up to $0.5 \mathrm{~cm}$ at base, clasts dominantly subangular to subround; light olive gray (5Y 6/1). Box 1 and Box 2.

$17.7-20.0 \mathrm{ft}$ : No recovery.

\section{0 - 24.0 ft: Run 6}

$20.0-23.0 \mathrm{ft}$ : No core recovery. Washings are SAND, quartz, feldspathic, $\mathrm{m}-\mathrm{vc}$, subangular to subround; light greenish gray (5GY 8/1).

23.0 - $24.0 \mathrm{ft}$ : No recovery. Geophysical log indicates gravel in this interval.

\section{0 - 29.0 ft: Run 7}

24.0 - $28.0 \mathrm{ft}$ : No recovery, except for three rounded quartz pebbles $1-2.5 \mathrm{~cm}$ in diameter. Geophysical log indicates gravel in this interval.

\section{Chowan River Formation}

28.0 - $29.0 \mathrm{ft}: \quad$ No recovery, top boundary picked from geophysical log.

\section{0 - 31.0 ft: Run 8}

29.0 - $30.4 \mathrm{ft}: \quad$ SAND, quartz, vf-f, silty; sparse chlorite (?) and phosphate sand; contains $20 \%$ or more shells and shell fragments, including a pectenid (not Chesapecten); dark greenish gray $(5 G 4 / 1)$. Box 2 .

$30.4-30.5 \mathrm{ft}: \quad$ No recovery.

\section{0 - 34.0 ft: Run 9}

30.5 - $33.7 \mathrm{ft}: \quad$ SAND, quartz, vf-f, silty; sparse chlorite (?) and phosphate sand; contains $20 \%$ or more shells and shell fragments; dark greenish gray $(5 G 4 / 1)$. Box 2.

$33.7-34.0 \mathrm{ft}$ : No recovery.

\section{0 - 40.0 ft: Run 10}

34.0 - $39.2 \mathrm{ft}: \quad$ SAND, quartz, vf-f, silty, with sparse chlorite(?) and phosphate; contains $20 \%$ or more shells and shell fragments; dark greenish gray (5G 4/1). Box 2 and Box 3.

$39.2-40.0 \mathrm{ft}$ : No recovery.

\section{0 - 50.0 ft: Run 11}

$40.0-42.3 \mathrm{ft}: \quad$ COQUINA, composed mostly of pelecypod and gastropod shells and shell fragments, contains less than $40 \%$ vf-f clean quartz sand, worn coral fragments present near base; sharp basal contact; greenish gray $(5 G 6 / 1)$. Box 3 . 


\section{Yorktown Formation}

$42.3-49.8 \mathrm{ft}: \quad$ SILT, clayey and well laminated, sparsely shelly, interbedded with SAND, quartz, vf, moderately shelly; silt layers thicken downward; dark greenish gray (6G $Y$ 4/1). Box 3 and Box 4.

$49.8-50.0 \mathrm{ft}: \quad$ No recovery.

\section{0 - 60.0 ft: Run 12}

$50.0-59.5 \mathrm{ft}: \quad$ SAND, quartz, vf, silty, finely micaceous, interbedded with SILT, clayey, vf sandy; shells sparse in sandy beds and absent in silt beds; foraminifera present in sand beds; clayey silts thinly bedded and bedding slightly wavy; dark greenish gray $(5 G Y 4 / 1)$. Box 4, Box 5, and Box 6.

$59.5-60.0 \mathrm{ft}: \quad$ No recovery.

\section{0 - 70.0 ft: Run 13}

$60.0-69.7 \mathrm{ft}: \quad$ SAND, quartz, vf, silty, finely micaceous, interbedded with SILT, clayey, vf sandy; sparse Mulinia shells in sandy beds and absent in silt beds; foraminifera present in sand beds; clayey silts thinly bedded and bedding slightly wavy; dark greenish gray $(5 G Y 4 / 1)$. Box 6 and Box 7.

$69.7-70.0 \mathrm{ft}$ : No recovery.

\section{0 - 80.0 ft: Run 14}

$70.0-80.0 \mathrm{ft}: \quad$ SAND, quartz, vf, silty, finely micaceous, interbedded with SILT, clayey, vf sandy; sparse Mulinia shells in sandy beds and absent in silt beds; foraminifera present in sand beds; clayey silts thinly bedded and bedding slightly wavy; more burrowed than beds above and burrows filled with vf quartz and glauconite sand; dark greenish gray $(5 G 4 / 1)$ with a few (organic rich?) interbeds of greenish black (5GY 2/1). Box 7 and Box 8 .

\section{0 - 90.0 ft: Run 15}

$80.0-83.8 \mathrm{ft}: \quad$ SAND, quartz, vf, silty, finely micaceous, interbedded with SILT, clayey, vf sandy; sparse Mulinia shells in sandy beds and absent in silt beds, foraminifera present in sand beds; clayey silts thinly bedded and bedding slightly wavy; burrows filled with vf quartz and glauconite sand; dark greenish gray $(5 G 4 / 1)$. Box 8 and Box 9.

83.8 - $83.9 \mathrm{ft}: \quad$ SAND, quartz (97\%), f-m, glauconite and phosphate?, f; moderately shelly; probable slight unconformity at base; dark greenish gray $(5 G 4 / 1)$. Box 9 .

$83.9-84.0 \mathrm{ft}: \quad$ SILT, clayey, micaceous, vf sandy; cheesy texture; pale olive $(10 Y 6 / 2)$. Box 9 .

$84.0-90.0 \mathrm{ft}: \quad$ No recovery. 


\section{0 - 95.0 ft: Run 16}

90.0 - $91.3 \mathrm{ft}: \quad$ SILT, clayey, micaceous, vf quartz sandy; cheese-like texture; pale olive (10 Y 6/2). Box 9.

91.3 - $92.4 \mathrm{ft}: \quad$ SAND, quartz, f-vc, very poorly sorted, subround, silty; contains scattered rounded quartz pebbles up to $1 \mathrm{~cm}$ in diameter and scattered rounded and polished phosphate granules up to $0.4 \mathrm{~cm}$ in diameter; sparsely shelly; dark greenish gray $(5 G 4 / 1)$. Box 9.

92.4 - $94.6 \mathrm{ft}: \quad$ SAND, quartz, vf-vc (modally $\mathrm{m}$ ), very poorly sorted, subangular to subround, silty, garnetiferous; very pebbly, subangular to subround quartz pebbles and subround to round phosphate pebbles up to $1 \mathrm{~cm}$ in diameter; about $30 \%$ shells and shell fragments; sharp basal contact; matrix greenish black (5G 2/1). Box 9.

\section{Beaufort Group}

94.6 - $94.9 \mathrm{ft}: \quad$ SAND, quartz, vf-m, subangular, silty, calcareous; greenish black (5GY2/1). Box 9. $94.9-95.0 \mathrm{ft}$ : No recovery.

\section{0 - 100.0 ft: Run 17}

95.0 - $95.4 \mathrm{ft}: \quad$ SANDSTONE, quartz, f-c (modally $\mathrm{m}$ ), calcite-cemented, semi-indurated; olive gray (5 Y 4/1). Box 9.

95.4 - $98.3 \mathrm{ft}: \quad$ SAND, quartz, f-c (modally $\mathrm{m}$ ), angular to subangular, includes $7-10 \%$ subround to round phosphate sand, with $<5 \%$ silt matrix, slightly calcareous; blue quartz grains (tr) and rare shell fragments; massive but with rare clay-silt stringers; high porosity, medium to low transmissivity; olive gray (5Y 4/1). Box 9 and Box 10.

$98.3-100.0 \mathrm{ft}: \quad$ No recovery

\section{0 - 110.0 ft: Run 18}

100.0 - $100.9 \mathrm{ft}:$ SANDSTONE, quartz, mostly f-m, some c, subangular, calcite-cemented, includes about $10 \%$ glauconite sand and about $10 \%$ silt matrix; faintly bedded; medium bluish gray $(5 B 5 / 1)$. Box 10 .

100.9 - $110.0 \mathrm{ft}:$ SAND, quartz, f-m, some c, subangular, includes about $5 \%$ glauconite sand decreasing downward to about $2-3 \%$ and about $10 \%$ silt matrix, slightly calcareous; massive texture; sparsely shelly; some small scattered clayballs in basal foot; rare blue quartz grains; 101.7 to 108.9 has more porosity and transmissivity than rest of this interval; olive gray (5Y 4/1). Box 10 and Box 11.

\section{0 - 120.0 ft: Run 19}

110.0 - $114.1 \mathrm{ft}:$ SAND, 97\% of sand fraction quartz and 3\% glauconite, f-m with sparse c, subround, with 15-20\% silt matrix; large shell fragment at $112.0 \mathrm{ft}$; subround to round quartz pebbles up to $1 \mathrm{~cm}$ in diameter increase in abundance downward; low porosity, very low transmissivity; olive gray (5Y 4/1). Box 11 and Box 12. 


\section{Unnamed formation (CC23 but lower than Peedee Fm. and higher than Donoho Creek Fm.)}

$114.1-118.3 \mathrm{ft}$ : SAND, $70 \%$ of sand fraction quartz and $30 \%$ glauconite, mostly $\mathrm{f}$ but some $\mathrm{m}$, with 5-10\% silt matrix; silvery mica (tr); massive to faintly bedded; some shell ghosts present; amber bleb about $2 \mathrm{~cm}$ long at $116.8 \mathrm{ft}$; low porosity and very low transmissivity; basal 0.3 feet contains abundant rounded phosphate, quartz, and shell fragment granules; dark greenish gray $(5 G 4 / 1)$. Box 12.

$118.3-120.0 \mathrm{ft}:$ No recovery.

120.0 - $121.5 \mathrm{ft}:$ Run 20

120.0 - $121.0 \mathrm{ft}$ : No recovery except for pseudo-discoidal pyrite nodule 24 x 22 x $8 \mathrm{~mm}$. Resistivity and gamma logs indicate break at $121.0 \mathrm{ft}$.

\section{Tar Heel Formation (Caddin Formation equivalent)}

$121.0-121.5 \mathrm{ft}:$ No recovery

\section{5 - 130.0 ft: Run 21}

121.1 - 123.9 ft: SILT, clayey, vf-f and rare m quartz sandy, slightly calcareous; $1-2 \%$ silvery mica; scattered subround quartz granules and pebbles up to $1 \mathrm{~cm}$ present, as well as wood chips up to $1 \mathrm{~cm}$ long and scattered pyrite nodules; rare shell fragments; burrow mottled, but some wispy bedding remains; olive black (5Y 2/1). Box 12.

123.9 - 124.0 ft: GRANULES, quartz, subround; small teleost vertebra present; olive black (5Y 2/1). Box 13.

124.0 - 130.0 ft: SILT, clayey, vf-f and rare m quartz sandy, slightly calcareous; $1-2 \%$ silvery mica; scattered subround quartz granules and pebbles up to $1 \mathrm{~cm}$ present, as well as wood chips up to $1 \mathrm{~cm}$ long and scattered pyrite nodules; rare shell fragments; burrow mottled but some wispy bedding remains; olive black (5Y 2/1). Box 13.

\section{0 - 140.0 ft: Run 22}

130.0 - $140.0 \mathrm{ft}$ : SILT, clayey, becoming more clayey downward, vf-f and rare m sandy, slightly calcareous; benthic foraminifera present; $3-4 \%$ silvery mica; scattered subround quartz granules and pebbles up to $1 \mathrm{~cm}$ present, as well as wood chips up to $1 \mathrm{~cm}$ long and scattered pyrite nodules; rare shell fragments; burrow mottled but some wispy bedding remains; olive black (5Y2/1). Box 13, Box 14 and Box 15.

\section{0 - 150.0 ft: Run 23}

140.0 - $145.2 \mathrm{ft}:$ SILT, clayey, vf-f and rare m sandy; more sandy downward, slightly calcareous; benthic foraminifera present; $3-4 \%$ silvery mica; scattered subround quartz granules and pebbles up to $1 \mathrm{~cm}$; wood chips up to $1 \mathrm{~cm}$ long and scattered pyrite nodules present; rare shell fragments; burrow mottled but some wispy bedding remains, 
burrows filled with f-m subround quartz (90\%) and glauconite (10\%) sand; olive black (5Y2/1). Box 15.

$145.2-150.0 \mathrm{ft}$ : SAND, 60-70\% of sand fraction quartz and 20-30\% glauconite and/or phosphate, $\mathrm{f}-$ $\mathrm{m}$ with rare c, subround, silty and clayey, slightly calcareous; rare pyrite nodules present; no obvious wood fragments; burrow mottling prominent; medium to low transmissivity; greenish black (5GY2/1). Box 15 and Box 16.

\section{0 - 160.0 ft: Run 24}

150.0 - $153.6 \mathrm{ft}$ : No recovery (due to fluctuating water pressure)

153.6 - $160.0 \mathrm{ft}$ : SAND, sand fraction $85-90 \%$ quartz and 10-15\% glauconite, vf-f, subround, silty, to SILT, vf-f sandy, about $15 \%$ clay, noncalcareous; $2-3 \%$ silvery mica; faint wispy wavy discontinuous bedding; some burrows present; sparse pyrite nodules to $0.5 \mathrm{~cm}$ in diameter; lower transmissivity than above; greenish black (5GY2/1). Box 16 and Box 17.

\section{Tar Heel Formation (Shepherd Grove Formation equivalent)}

\section{0 - 162.0 ft: Run 25}

160.0 - $161.8 \mathrm{ft}:$ SAND, quartz, f-m grading down to $\mathrm{m}-\mathrm{c}$ with rare vc, with about $5 \%$ silt matrix, noncalcareous; silvery mica (tr) and blue quartz grains (tr); massive; olive gray ( $5 Y$ 4/1) grading down to light olive gray (5Y 6/1). Box 17.

161.8 - $162.0 \mathrm{ft}$ : No recovery.

\section{0 - $170.0 \mathrm{ft}:$ Run 26}

162.0 - $167.0 \mathrm{ft}:$ SAND, quartz, f-vc, very poorly sorted, subround to round, with less than $2 \%$ silt matrix; mica (tr), garnet (tr), and blue quartz (tr); massive; light olive gray (5Y 6/1). Box 17 and Box 18.

167.0 - $170.0 \mathrm{ft}$ : No recovery. Geophysical logs suggest transmissive gravelly sand.

\section{0 - 180.0 ft: Run 27}

170.0 - $170.7 \mathrm{ft}:$ SAND, quartz, f-vc, subangular to subround, with 3-5\% silt and clay matrix; contains subangular quartz pebbles and wood chips up to $0.5 \mathrm{~cm}$ in diameter; light olive gray $(5 Y 6 / 1)$. Box 18.

170.7 - $172.0 \mathrm{ft}$ : SAND, quartz, vf-f, angular to subangular, with 5-7\% silt and clay matrix; silvery mica (tr) and rutilated quartz (tr); thin stringers of clay-silt define faint bedding; light olive gray (5Y 6/1). Box 18.

172.0 - $172.1 \mathrm{ft}:$ SILT, clayey, noncalcareous; olive gray $(5 Y 4 / 1)$. Box 18.

172.1 - $173.3 \mathrm{ft}$ : SAND, quartz, vf-m, angular to subangular, with 3-5\% silt and clay matrix; contains rounded lignite clasts up to $1 \mathrm{~cm}$ in diameter; silvery mica (tr) and rutilated quartz (tr); light olive gray (5Y 6/1). Box 18.

173.3 - $180.0 \mathrm{ft}$ : No recovery. Geophysical logs suggest transmissive gravelly sands in this interval. 


\section{0 - 190.0 ft: Run 28}

180.0 - $184.2 \mathrm{ft}$ : No recovery. Geophysical logs suggest transmissive gravelly sand in this interval.

184.2 - $184.8 \mathrm{ft}$ : SILT, clayey, vf-f quartz sandy, subround; $1 \%$ silvery mica, $1 \%$ fine lignite; poorly bedded; olive gray (5Y 4/1). Box 18.

184.8 - $185.5 \mathrm{ft}$ : SAND, quartz, vf and some f; 3-5\% silvery mica; lignite and clay concentrated in discontinuous stringers defining wavy planar bedding; light olive gray $(5 Y 6 / 1)$. Box 18.

185.5 - $185.8 \mathrm{ft}$ : SILT, contains less than $10 \%$ vf quartz sand, noncalcareous; thin flaser bedding; olive gray (5Y 4/1) and light olive gray (5Y 6/1) interlaminated. Box 18.

185.8 - $188.3 \mathrm{ft}$ : SAND, quartz, f-m, subround, grading down to f-vc and very poorly sorted, subangular, with 5\% silt and clay matrix; lignite (tr), silvery mica (tr), and blue quartz (tr); subround quartz pebbles up to $1.5 \mathrm{~cm}$ in diameter in basal $0.3 \mathrm{foot}$; sharp basal contact; olive gray (5Y 4/1). Box 18 and Box 19.

\section{Cape Fear Formation (subaerial facies)}

188.3 - $190.0 \mathrm{ft}$ : SILT, very clayey; contains $1 \%$ vf round quartz sand, chloritic(?); faintly bedded and locally blocky, dry, blocky intervals appear to be ped structures; olive black (5Y 2/1) mottled brownish gray (5YR 4/1) and olive gray (5Y 4/1). Box 19.

\section{0 - 200.0 ft: Run 29}

190.0 - $194.5 \mathrm{ft}:$ SILT, clayey to very clayey; some intervals poorly bedded and others blocky; scattered pyrite blebs present from 192.3 to $193.2 \mathrm{ft}$; local blebs of fine sand abundant, possibly root ghosts; no transmissivity; olive gray $(5 Y 4 / 1)$ grading about $192.6 \mathrm{ft}$ through light olive gray (5Y 6/1) mottled light greenish gray $(5 G 6 / 1)$, then grading about 194.2 to light greenish gray $(5 G 6 / 1)$ streaked and blotched moderate brown (5YR 4/4). Box 19.

$194.5-200.0 \mathrm{ft}$ : No recovery.

\section{0 - 205.0 ft: Run 30}

200.0 - $204.2 \mathrm{ft}$ : SILT, very clayey, contains 3\% vf quartz sand; silvery mica (tr); ped structures and poorly developed layering locally present; intensely color-mottled, mostly olive gray (5Y 4/1), grayish red (10R 4/2), light olive gray $(5 Y 6 / 1)$, moderate yellowish brown (10YR 5/4), and dark reddish brown (10R 3/4). Box 19 and Box 20.

204.2 - $205.0 \mathrm{ft}$ : No recovery.

\section{0 - 210.0 ft: Run 31}

205.0 - $205.3 \mathrm{ft}$ : SILT, very clayey, contains $~ 3 \%$ vf angular quartz sand; silvery mica (tr); ped structures and poorly developed layering locally present; intensely color-mottled, mostly olive gray $(5 Y 4 / 1)$, grayish red $(10 R 4 / 2)$, light olive gray $(5 Y 6 / 1)$, moderate yellowish brown (10YR 5/4), and dark reddish brown (10R 3/4). Box 20.

205.3 - $207.9 \mathrm{ft}$ : SILT, very clayey, contains $\sim 5 \%$ vf-f angular quartz sand; massive, very dense, conchoidal fracture; soil fabric includes root ghosts; irregular contact with bed 
below; dominantly dark greenish gray (5GY 4/1) mottled dark reddish brown (10R 3/4) and moderate yellowish brown (10 Y R5/4). Box 20.

207.9 - $209.6 \mathrm{ft}$ : SILT, contains $\sim 25 \%$ vf-m subangular to angular quartz sand; $\sim 1-2 \%$ silvery mica, $\sim 1 \%$ vf-f dark organic fragments; massive, subangular quartz granules concentrated at base; light olive gray (5Y 6/1) mottled moderate yellowish brown (10YR 5/4). Box 20 and Box 21.

209.6 - $209.9 \mathrm{ft}$ : SILT, very clayey, contains 5\% vf-f angular quartz sand; massive, very dense, conchoidal fracture, soil fabric includes root ghosts; dominantly dark greenish gray (5GY 4/1) mottled dark reddish brown (10R 3/4) and moderate yellowish brown (10YR 5/4). Box 21.

209.9 - $210.0 \mathrm{ft}$ : No recovery.

\section{0 - 220.0 ft: Run 32}

210.0 - $211.0 \mathrm{ft}: \quad$ SILT, very clayey, contains $\sim 5-7 \%$ f-m subangular quartz sand; $~ 1-2 \%$ silvery mica; very dense; faintly bedded, root structures visible; dominantly dark greenish gray (5GY 4/1) mottled moderate yellowish brown (10YR 5/4). Box 21.

211.0 - $214.3 \mathrm{ft}$ : SILT, clayey, contains 15\% f-m subangular quartz sand; planar bedded; light olive gray (5Y 6/1) mottled olive gray (5Y 4/1); grading down to SAND, quartz, f-c, subangular, silty; some subangular quartz granules near base; 2-3\% silvery mica; cross-bedded, dark heavy minerals or graphite concentrated on some bedding planes; greenish gray (5GY 6/1) mottled moderate yellowish brown (10YR 5/4). Box 21.

214.3 - $216.6 \mathrm{ft}$ : SILT, clayey, contains $\sim 5-7 \% \mathrm{f}-\mathrm{m}$ subangular quartz sand; grades into bed below; moderate yellowish brown (10YR 5/4) mottled light olive gray $(5 Y 6 / 1)$, dark reddish brown (10R 3/4), and moderate brown (5YR 4/4). Box 21.

216.6 - $217.6 \mathrm{ft}$ : SAND, quartz, f, subangular, with clay and silt matrix; grades into bed below; greenish gray $(5 G Y 6 / 1)$ mottled dark reddish brown (10R 3/4) and moderate brown (5YR 4/4). Box 21 and Box 22.

217.6 - $218.9 \mathrm{ft}:$ SAND, quartz, feldspathic?, f-vc, subangular, with clay and silt matrix, subangular to subround, quartz granular; granules increase in abundance downward to about $3 \%$ at base, mostly quartz, smoky and rutilated; silvery mica (tr) near base; greenish gray $(5 G Y$ 6/1) mottled dark reddish brown (10R 3/4) and moderate brown (5YR 4/4). Box 22.

218.9 - $220.0 \mathrm{ft}:$ No recovery.

\section{0 - 230.0 ft: Run 33}

220. 0 - $223.6 \mathrm{ft}:$ SILT, clayey, containing vf-m quartz sand, grading to SAND, quartz, f-vc, with subangular to subround granules of quartz and feldspar present; up to $4 \%$ silvery mica present locally; poorly bedded, some bedding contorted; $1 \mathrm{~cm}$-thick flattened lignitic branch or log cored at base; varicolored but mostly greenish gray $(5 G Y 6 / 1)$, dark reddish brown (10R 3/4) moderate brown (5YR 4/4), and very dusky red (10R $2 / 2$ ); thin layer $1.5 \mathrm{~cm}$-thick immediately above log or branch at base is silt, $\mathrm{f}-\mathrm{c}$ subangular quartz sandy, dark gray (N 3). Box 22.

$223.6-230.0 \mathrm{ft}$ : No recovery. Geophysical logs suggest this interval is moderately transmissive sand. 


\section{0 - 240.0 ft: Run 34}

230.0 - $230.8 \mathrm{ft}$ : SAND, quartz, f-c, subangular to subround; lignitic branch or log cored at 230.1 to 230.2; below lignite, sand becomes quartz granular and contains clayballs; faint cross-bedding; dark greenish gray ( $5 G Y 4 / 1)$ grading down to light olive gray (5Y 6/1). Box 22.

230.8 - $233.9 \mathrm{ft}$ : SILT, very clayey, contains 3\% f quartz sand; silvery mica (tr); mostly massive, with some ped structures and root ghosts present; greenish gray $(5 G 6 / 1)$ streaked medium dark gray $(N 4)$ and greenish gray $(5 G Y 6 / 1)$, grading at about $232.2 \mathrm{ft}$ to varicolored blotches of dark reddish brown (10R 3/4), moderate reddish brown (10R 4/6), pale red (5R 6/2), and moderate brown (5YR 4/4). Box 22 and Box 23.

233.9 - $240.0 \mathrm{ft}$ : No recovery.

\section{0 - 250.0 ft: Run 35}

240.0 - $245.0 \mathrm{ft}:$ SAND, quartz, vf-f grading down to f-m, subangular, silt matrix; 1\% silvery mica; faintly bedded; grades into bed below; greenish gray $(5 G Y 6 / 1)$ blotched and streaked moderate reddish brown (10R 4/6), moderate yellowish brown (10YR 5/4), pale red (10R 6/2), and dusky yellow (5Y 6/4). Box 23.

245.0 - $248.2 \mathrm{ft}:$ SAND, quartz, f-vc grading down to f-c, subangular, very poorly sorted, with subround quartz granules and $\sim 7 \%$ silt and clay matrix; faintly cross-bedded; greenish gray $(5 G Y 6 / 1)$ blotched and streaked moderate reddish brown $(10 R 4 / 6)$, moderate yellowish brown (10YR 5/4), pale red (10R 6/2), and dusky yellow (5Y 6/4). Box 23 and Box 24.

$248.2-250.0 \mathrm{ft}:$ No recovery.

\section{0 - 260.0 ft: Run 36}

250.0 - 251. $7 \mathrm{ft}$ : SAND, quartz, vf-f, subround, silty matrix; occasional quartz granules and coarse grains present; silvery mica (tr); faintly bedded; moderate brown $(5 Y R 4 / 4)$ mottled moderate yellowish brown (10YR 5/4), moderate olive brown ((5Y 4/4), and grayish olive $(10 Y 4 / 2)$, grading down to dusky yellowish green $(5 G Y 5 / 2)$ mottled pale red $(5 R 6 / 2)$. Box 24.

\section{Cape Fear Formation (marine facies) ( = Pleasant Creek Formation?)}

251.7 - $256.3 \mathrm{ft}$ : SILT, very clayey, contains $\sim 3-5 \%$ vf angular quartz sand; faintly banded with blocky ped structures; no transmissivity; dark greenish gray $(5 G Y 4 / 1)$ and greenish gray $(5 G Y 6 / 1)$ mottled brownish gray $(5 Y \mathrm{R} 4 / 1)$ and moderate yellowish brown (10YR 4/2). Box 24 and Box 25.

$256.3-260.0 \mathrm{ft}$ : No recovery.

\section{0 - 270.0 ft: Run 37}

260.0 - $260.9 \mathrm{ft}$ : SILT, clayey, contains vf subangular quartz sand, grading rapidly to SAND, quartz, slightly feldspathic f-vc, subround; subround quartz and lignite granules moderately abundant near base; bright lemon yellow sand-size grain observed; olive gray ( $5 \mathrm{Y}$ 4/1). Box 25. 
260.9 - 262.2 ft: SAND, quartz, vf-f, subround, with 3-5\% silt matrix; 2\% silvery mica; faintly bedded, lignite grains define bedding planes; light-olive-gray (5Y6/1). Box 25.

$262.2-270.0 \mathrm{ft}$ : No recovery. Geophysical logs suggest this interval is transmissive sand.

\section{0 - 276.0 ft: Run 38}

$270.0-272.6 \mathrm{ft}$ : SAND, quartz, f-c but modally f-m, subangular, with $<5 \%$ silt and clay matrix; $1-2 \%$ silvery mica; massive to locally faintly bedded; thin lignite layers present; medium to low porosity, moderate transmissivity; light olive gray (5Y 5/2). Box 25.

272.6 - $276.0 \mathrm{ft}$ : No recovery. Geophysical logs suggest this interval is transmissive sand.

\section{0 - 283.0 ft: Run 39}

276.0 - $277.2 \mathrm{ft}$ : SAND, quartz, f-c grading down to $\mathrm{f}-\mathrm{vc}$, subangular, with $<5 \%$ silt and clay matrix; $1-2 \%$ silvery mica; massive; thin lignite layers present, more abundant near base; subangular quartz granules and pyrite nodules up to $1 \mathrm{~cm}$ in diameter present near base; medium to low porosity, moderate transmissivity; light olive gray ( $5 Y 5 / 2)$. Box 25.

277.2 - $278.6 \mathrm{ft}$ : SAND, quartz, f-vc, angular to subangular, contains subangular quartz granules and $\sim 10 \%$ silt and clay matrix; silvery mica (tr) and rose quartz (tr); lignite pieces and stringers scattered throughout; sand-filled burrows present at top; abundant subangular to round clay clasts ranging from granule size up to $2 \mathrm{~cm}$ in diameter; matrix dark gray $(N 3)$, clay clasts greenish gray (5GY 6/1). Box 25 and Box 26.

$278.6-282.4 \mathrm{ft}$ : SAND, quartz, f, contains either $\sim 7 \%$ or $\sim 50 \%$ bimodal silt and clay matrix concentration; clean and clayey sand layers interbedded, some beds planar and others cross-bedded; thin lignite layers define bedding planes; locally up to $7 \%$ silvery mica and up to $10 \%$ lignite; submarine delta?; clayey sands medium dark gray $(N 4)$, clean sands light olive gray $(5 Y 6 / 1)$. Box 26.

$282.4-283.0 \mathrm{ft}$ : No recovery.

\section{0 - 290.0 ft: Run 40}

283.0 - $283.4 \mathrm{ft}:$ SAND, quartz, vf-f, subround, with clayey matrix; silvery mica locally up to $10-$ $12 \%$; cross-bedded, bedding defined by lignite laminae that thicken downward; medium dark gray $(N 4)$. Box 26.

$283.4-283.8 \mathrm{ft}$ : SAND, quartz, vf-f, subangular; 3\% silvery mica, scattered grains of lignite; wispy bedding; light olive gray (5Y 6/1). Box 26.

283.8 - $284.8 \mathrm{ft}:$ SAND, quartz, vf-f, subround, with clayey matrix; silvery mica locally up to $10-$ $12 \%$; cross-bedded, bedding defined by lignite laminae; grades into bed below; medium dark gray $(N 4)$. Box 26.

284.8 - $285.9 \mathrm{ft}:$ SAND, quartz, vf-f, subangular; 3\% silvery mica, scattered grains of lignite; wispy bedding; lignite lense at 285.6 to $285.7 \mathrm{ft}$; light olive gray (5Y 6/1). Box 26.

$285.9-290.0 \mathrm{ft}$ : No recovery. 


\section{0 - 300.0 ft: Run 41}

290.0 - $291.4 \mathrm{ft}:$ SAND, quartz, f-m, subangular, with $<5 \%$ silt and clay matrix; silvery mica locally up to $5 \%$; sparse disseminated lignite chips define faint bedding; light olive gray ( $5 Y$ 6/1). Box 26 and Box 27.

291.4 - $291.5 \mathrm{ft}$ : SAND, quartz, f, subangular, with clay and silt matrix; medium gray (N 5). Box 27.

291.5 - $292.5 \mathrm{ft}$ : SAND, quartz, f-m, subangular, with <5\% silt and clay matrix; silvery mica locally up to 5\%; sparse disseminated lignite chips define faint bedding; light olive gray (5Y 6/1). Box 27.

292.5 - $292.9 \mathrm{ft}:$ SILT, very clayey, contains $~ 10 \%$ vf-f subangular quartz sand; 5\% silvery mica; well laminated, lamination defined by thin stringers of quartz sand; medium dark gray $(N 4)$. Box 27.

292.9 - $295.9 \mathrm{ft}$ : SAND, quartz, f, subangular, with <5\% silt and clay matrix; 1\% silvery mica; sparse disseminated lignite chips define faint bedding, some inclined; light olive gray $(5 Y 6 / 1)$. Box 27.

295.9 - $300.0 \mathrm{ft}$ : No recovery.

\section{0 - 310.0 ft: Run 42}

300.0 - 300.1 ft: SAND, quartz, f, subangular, with <5\% silt and clay matrix; 1\% silvery mica; sparse disseminated lignite chips define faint bedding, some inclined; light olive gray (5Y 6/1). Box 27.

300.1 - $302.3 \mathrm{ft}$ : SILT, clayey, contains $\sim 40 \%$ vf subangular quartz sand; $\sim 1 \%$ silvery mica; $~ 2 \%$ flattened lignite chips up to $5 \mathrm{~cm}$ in length disseminated throughout; poorly bedded to burrow mottled; light-olive-gray $(5 Y$ 6/1) in more sandy areas and brownish gray $(5 Y R 4 / 1)$ in more clay-rich areas. Box 27.

302.3 - $308.1 \mathrm{ft}:$ SILT, very clayey, contains $\sim 1 \%$ lignite sand fraction; massive; basal 0.3 foot has visible green-filled burrows and about $3 \%$ lignite sand; basal contact churned, possibly due to dewatering; brownish gray (5YR 4/1). Box 27 and Box 28.

308.1 - $308.3 \mathrm{ft}$ : SAND, quartz, f-m, subround, with glauconite (tr); burrow mottled; dusky yellowish green $(5 G Y 5 / 2)$. Box 28.

308.3 - 308.5 ft: SILT, very clayey, noncalcareous; massive; greenish gray (5G 6/1). Box 28 .

308.5 - $309.8 \mathrm{ft}$ : SAND, quartz, f-c, poorly sorted, subround, contains < $1 \%$ subround quartz granules, noncalcareous; up to $5 \%$ chlorite flakes and glauconite pellets in burrows; silvery mica (tr); grayish green $(10 G 4 / 2)$ that faded rapidly to greenish gray $(5 G Y 6 / 1)$. Box 28.

309.8 - $310.0 \mathrm{ft}$ : No recovery.

\section{0 - 315.0 ft: Run 43}

310.0 - 313.1 ft: SAND, quartz, f-m, subround, grading down to vf-f, subangular to subround, some intervals have abundant clay and silt matrix; silvery mica (tr) at top increasing in abundance to $3 \%$ near base; chloritic; faintly bedded to burrow mottled, possible faint dewatering structures present; dusky yellowish green $(5 G Y 5 / 2)$ and grayish yellow green $(5 G Y 6 / 2)$ grading down to dusky yellowish green $(5 G Y 5 / 2)$ and brownish gray (5YR 4/1), all mottled olive gray (5Y 4/1). Box 28 and Box 29. 
313.1 - 315.0 ft: SAND, quartz, vf-f, subangular to subround, interbedded with SILT, very clayey; silt zones predominate; clays swell; silty zones brownish gray $(5 Y R 4 / 1)$ and sandy zones yellowish gray $(5 Y 7 / 2)$ to light olive gray $(5 Y 7 / 1)$. Box 29.

\section{0 - 320.0 ft: Run 44}

315.0 - $319.8 \mathrm{ft}:$ SAND, quartz, f-m, subangular, with 5\% silt and clay matrix; 1\% silvery mica; light olive gray $(5 Y 6 / 1)$; massive except for thin dark sand lenses up to $0.5 \mathrm{~cm}$-thick, containing $\sim 7 \% \mathrm{~m}-\mathrm{c}$, subround phosphate and phlogopite (tr), garnet (tr), rutilated quartz (tr), and pyrite; medium dark gray (N 4). Box 29 and Box 30.

319.8 - $320.0 \mathrm{ft}$ : No recovery.

\section{0 - 330.0 ft: Run 45}

320.0 - $321.5 \mathrm{ft}:$ SAND, quartz, f-c, angular to subangular, with 5\% silt and clay matrix; silvery mica (tr); well bedded at top fading down to massive by base; modally coarser at bottom with sparse subround quartz granules; light olive gray (5Y 6/1). Box 30 .

321.5 - $321.7 \mathrm{ft}$ : SILT, very clayey, no sand; massive, sticky; olive gray (5Y 4/1). Box 30 .

321.7 - $323.3 \mathrm{ft}$ : SAND, quartz, f-c, angular to subangular grading down to f-vc, subangular, with $\sim 5 \%$ silt and clay matrix; silvery mica (tr); well bedded at top fading down to massive by base; modally coarser at bottom with sparse subround quartz granules; grades rapidly downward into next bed; light olive gray (5Y 6/1). Box 30 .

323.3 - $325.5 \mathrm{ft}:$ SAND, quartz, f-vc, with $\sim 5 \%$ angular to subround quartz granules and pebbles and $<5 \%$ silt and clay matrix; silvery mica (tr), blue quartz (tr), and rose quartz (tr); thin clay stringers up to $2 \mathrm{~cm}$-thick present from 324.2 to $325.5 \mathrm{ft}$; sand light olive gray $(5 Y 6 / 1)$, clay stringers dark greenish gray $(5 G Y 4 / 1)$. Box 30 .

325.5 - $328.6 \mathrm{ft}$ : SAND, quartz, f-m coarsening down to $\mathrm{m}-\mathrm{vc}$; similar to cycle from 321.7 to $325.5 \mathrm{ft}$, but with the addition of zones with abundant lignite clasts up to $0.5 \mathrm{~cm}$ in diameter; light olive gray (5Y 6/1). Box 30 and Box 31.

328.6 - $328.8 \mathrm{ft}$ : GRAVEL, quartz clasts up to $1.5 \mathrm{~cm}$ in diameter, angular to subangular, with very coarse to granule-size quartz sand matrix; light olive gray $(5 Y 6 / 1)$. Box 31 .

\section{Cape Fear Formation (subaerial facies)}

328.8 - $330.0 \mathrm{ft}$ : SILT, clayey, contains vf-f subround quartz sand in pockets and blebs; relict root structures present, no obvious bedding; olive gray (5Y 4/1). Box 31.

\section{0 - 340.0 ft: Run 46}

330.0 - $332.8 \mathrm{ft}:$ SILT, clayey, contains 1-2\% vf-f subround quartz sand; silvery mica (tr); banded to blocky ped structures and root structures present; olive gray $(5 Y 4 / 1)$ grading down to olive gray mottled greenish gray $(5 G Y 6 / 1)$. Box 31 .

332.8 - $333.8 \mathrm{ft}$ : DISTURBED CORE, due to re-drilling this run (core slipped out of barrel and had to be retrieved). Box 31.

333.8 - $335.7 \mathrm{ft}$ : SILT, clayey, contains 1-2\% vf-f subround quartz sand; silvery mica (tr); banded to blocky ped structures and root structures present; greenish gray $(5 G Y 6 / 1)$ mottled dark reddish brown (10R 3/4) and moderate yellowish brown (10YR 5/4). Box 31 and Box 32. 
335.7 - $335.8 \mathrm{ft}:$ DISTURBED CORE, due to re-drilling this run. Box 32.

335.8 - $336.5 \mathrm{ft}$ : SILT, clayey, contains $1-2 \%$ vf-f subround quartz sand; silvery mica (tr); banded to blocky ped structures and root structures present; greenish gray $(5 G Y 6 / 1)$ mottled dark reddish brown (10R 3/4) and moderate yellowish brown (10YR 5/4). Box 32 .

336.5 - $337.6 \mathrm{ft}$ : DISTURBED CORE, due to re-drilling this run. Box 32.

337.6 - $337.8 \mathrm{ft}$ : SILT, clayey, contains $1-2 \%$ vf-f subround quartz sand; silvery mica (tr); banded to blocky ped structures and root structures present; greenish gray (5GY 6/1) mottled dark reddish brown (10R 3/4) and moderate yellowish brown (10YR 5/4). Box 32.

$337.8-340.0 \mathrm{ft}$ : No recovery.

\section{0 - 350.0 ft: Run 47}

340.0 - $342.1 \mathrm{ft}:$ SILT, clayey, contains $\sim 10 \%$ vf-f subround quartz sand; $~ 3 \%$ silvery mica; wavy laminated; light olive gray $(5 Y 6 / 1)$. Box 32 .

342.1 - 342.5 ft: SAND, quartz, vf-f, subround; 5-7\% silvery mica; prominently cross-bedded; light olive gray $(5 Y 6 / 1)$. Box 32 .

342.5 - $343.2 \mathrm{ft}$ : SILT, clayey, contains $\sim 45 \%$ vf-f subround quartz sand; $~ 3 \%$ silvery mica; wavy laminated; light olive gray $(5 Y 6 / 1)$. Box 32 .

$343.2-348.0 \mathrm{ft}$ : SAND, f-c grading down to $\mathrm{m}$-vc, subangular to subround, poorly sorted, with $\sim 5 \%$ silt and clay matrix; interlaminated faint cross-bedding and wavy bedding; lignite grains define bedding planes, often discontinuous; interval includes four fining upward sequences (343.2 - 344.1, 344.1 - 345.5, 345.5 - 346.3, and 346.3 - 348.0); greenish gray $(5 G Y 6 / 1)$. Box 32 and Box 33 .

348.0 - $349.5 \mathrm{ft}$ : SILT, clayey, contains $\sim 10 \%$ vf subangular quartz sand in blebs; $\sim 2 \%$ silvery mica; blocky ped and root structures visible; no transmissivity; medium dark gray $(N 4)$. Box 33.

$349.5-350.0 \mathrm{ft}:$ No recovery.

\section{0 - 360.0 ft: Run 48}

$350.0-352.1 \mathrm{ft}$ : SILT, clayey, contains $\sim 10 \%$ vf subangular quartz sand in blebs; $\sim 2 \%$ silvery mica; blocky ped and root structures visible; gradational with bed below; no transmissivity; olive gray (5Y 4/1) mottled dusky red (5R 3/4) and moderate brown $(5 Y R 4 / 4)$. Box 33.

352.1 - $353.3 \mathrm{ft}:$ SILT, clayey, contains $~ 10 \%$ vf subangular quartz sand in blebs; $2 \%$ silvery mica; blocky ped and root structures visible; no transmissivity; greenish gray $(5 G Y 6 / 1)$ mottled moderate yellowish brown (10YR 5/4) and dark reddish brown (10R 3/4). Box 33.

$353.3-360.0 \mathrm{ft}$ : No recovery.

\section{0 - 363.0 ft: Run 49}

$360.0-363.0 \mathrm{ft}:$ SILT, clayey, contains $\sim 5 \%$ quartz sand in blebs and stringers; $\sim 1-2 \%$ silvery mica; paleosol with root structures, lower part brecciated (paleoliquefaction shattering?); tight and dry; varicolored including greenish gray $(5 G Y 6 / 1)$, dark reddish brown $(10 R 3 / 4)$, pale red $(5 R 6 / 2)$, dusky red ( $5 R 3 / 4)$, olive gray (5Y 4/1), dark gray $(N 3)$, and moderate brown $(5 Y R 4 / 4)$. Box 34 . 


\section{0 - 370.0 ft: Run 50}

363.0 - $370.0 \mathrm{ft}:$ SILT, clayey, contains $\sim 10 \%$ quartz sand in blebs and stringers; $\sim 1-2 \%$ silvery mica; paleosol with root structures; tight and dry; varicolored including greenish gray ( $5 G Y$ $6 / 1)$, dark reddish brown (10R 3/4), pale red (5R 6/2), dusky red $(5 R 3 / 4)$, olive gray (5Y 4/1), dark gray (N 3), and moderate brown (5YR 4/4). Box 34 and Box 35.

\section{0 - 380.0 ft: Run 51}

370.0 - $375.2 \mathrm{ft}:$ SILT, clayey, contains $~ 10 \%$ quartz sand in blebs and stringers; silvery mica locally up to $\sim 5 \%$; paleosol with root structures; tight and dry; dark reddish brown (10R 3/4) grading down to moderate brown $(5 Y R$ 4/4) mottled and streaked grayish green $(5 G$ $5 / 2$ ) and pale green (10G 6/2). Box 35 and Box 36.

375.2 - $375.5 \mathrm{ft}:$ SAND, quartz, modally $\mathrm{m}$ but vf-c, subround, with $~ 7 \%$ silt and clay matrix; dark greenish gray $(5 G Y 4 / 1)$. Box 36 .

375.5 - $376.2 \mathrm{ft}$ : SILT, clayey, contains $\sim 10 \%$ quartz sand in blebs and stringers; silvery mica locally up to $~ 5 \%$; paleosol with root structures; tight and dry; moderate brown (5YR 4/4) mottled and streaked grayish green $(5 G 5 / 2)$ and pale green $(10 G 6 / 2)$. Box 36 .

376.2 - $376.8 \mathrm{ft}$ : SAND, quartz, modally c but f-vc, subround, with <5\% silt matrix; greenish gray $(5 G Y 6 / 1)$. Box 36.

376.8 - $380.0 \mathrm{ft}$ : SILT, contains 5-10\% quartz sand; massive, color patterns may reflect root ghosts; medium gray $(N 5)$ mottled moderate reddish brown $(10 R 4 / 6)$, sparse mottles of blackish red (5R 2/2) and dark reddish brown (10R 3/4) present below $377.7 \mathrm{ft}$. Box 36.

\section{0 - 390.0 ft: Run 52}

380.0 - $382.7 \mathrm{ft}:$ SILT, contains 5-10\% quartz sand; massive, color patterns may reflect root ghosts; medium gray $(N 5)$ mottled moderate reddish brown $(10 R 4 / 6)$ blackish red $(5 R 2 / 2)$ and dark reddish brown (10R 3/4). Box 36 and Box 37.

382.7 - 384.4 ft: SAND, quartz, vf-f, subangular, with 7-10\% silt and clay matrix; $~ 1 \%$ silvery mica, $\sim 1 \%$ finely disseminated lignite, $\sim 1 \%$ glauconite or darker than normal chlorite; faintly bedded and mottled; dusky yellowish green $(5 G Y 5 / 2)$ mottled light olive gray (5Y 6/1). Box 37.

384.4 - $390.0 \mathrm{ft}$ : No recovery. Short-normal log suggests this interval is sand.

\section{0 - 400.0 ft: Run 53}

390.0 - $398.9 \mathrm{ft}:$ SILT, clayey, contains 5\% vf-f subangular quartz sand; silvery mica (tr); blocky ped texture with root ghosts; grades into bed below; olive gray $(5 Y 4 / 1)$ mottled and blotched light olive gray (5Y 6/1), grayish red (10R 4/2), very dusky red (10R $2 / 2)$, and moderate yellowish brown (10YR 5/4). Box 37 and Box 38.

398.9 - $400.0 \mathrm{ft}$ : SILT, clayey, contains $~ 15-20 \% \mathrm{f}-\mathrm{vc}$ subangular to subround quartz sand; silvery mica (tr); blocky ped texture with root ghosts; olive gray $(5 Y 4 / 1)$ mottled and blotched light olive gray $(5 Y 6 / 1)$, grayish red (10R 4/2), very dusky red $(10 R 2 / 2)$, and moderate yellowish brown (10YR 5/4). Box 38. 


\section{0 - 410.0 ft: Run 54}

400.0 - $406.5 \mathrm{ft}:$ SILT, contains 20\% f-vc subround quartz sand; 3-5\% scattered quartz granules, garnet (tr); massive, blocky, root structures visible above $401.5 \mathrm{ft}$; sand fraction coarsens downward; very tight matrix, some porosity but no transmissivity; moderate yellowish brown (10YR 5/4) mottled and blotched dark yellowish orange (10YR 6/6), dark reddish brown (10R 3/4), and light olive gray (5Y 6/1). Box 38 and Box 39.

406.5 - $407.7 \mathrm{ft}$ : SAND, quartz, f-vc, subround, contains 3-5\% subangular to subround quartz granules; one subangular quartz pebble $1 \mathrm{~cm}$ in diameter; massive; moderate yellowish brown 10YR 5/4) mottled and blotched dark yellowish orange (10YR 6/6), dark reddish brown (10R 3/4), and light olive gray (5Y 6/1). Box 39.

$407.7-410.0 \mathrm{ft}$ : No recovery.

\section{0 - 420.0 ft: Run 55}

410.0 - $411.3 \mathrm{ft}:$ SAND, quartz, f-m grading down to f-c, subround, with $~ 10 \%$ silt and clay matrix; silvery mica (tr); no granules or pebbles; sharp basal contact; dark reddish brown (10R 3/4) mottled moderate brown (5YR 4/4) and dusky red (5R 3/4). Box 39.

411.3 - $412.2 \mathrm{ft}$ : SILT, very clayey, contains $\sim 7 \%$ vf-f subround quartz sand; ped structures and root ghosts present; semi-indurated iron-oxide nodule at base; clay greenish gray $(5 G 6 / 1)$ mottled dark reddish brown (10R 3/4), nodule dark reddish brown. Box 39.

412.2 - $420.0 \mathrm{ft}$ : No recovery. Iron nodule probably blocked drill bit.

\section{0 - 430.0 ft: Run 56}

420.0 - $423.8 \mathrm{ft}$ : SAND, quartz, f-m, subangular to subround, grading down to f-c, subangular, very poorly sorted, contains scattered quartz granules and $\sim 10 \%$ silt and clay matrix; quartz pebbles up to $1 \mathrm{~cm}$ in diameter at base; light olive gray $(5 Y 6 / 1)$ mottled dark reddish brown (10R 3/4) grading down to dark yellowish orange at $423.5 \mathrm{ft}$. Box 39 and Box 40.

423.8 - $426.0 \mathrm{ft}$ : SILT, very clayey, grading by $423.9 \mathrm{ft}$ to SILT, clayey, contains $~ 20 \% \mathrm{f}-\mathrm{m}$ subangular quartz sand, and then to SAND, quartz, f-c, subangular, poorly sorted, with $\sim 10 \%$ silt and kaolinite clay matrix; root structures visible at top of bed; between yellowish gray and light olive gray (5Y 7/1) mottled dark yellowish orange (10YR 6/6) and moderate reddish orange (10R 6/6). Box 40.

426.0 - $430.0 \mathrm{ft}$ : No recovery; mislabeled on picture as " 316.0 - 320.0”.

\section{0 - 440.0 ft: Run 57}

430.0 - $432.5 \mathrm{ft}:$ SAND, quartz, m-vc, subangular to subround, with 40-50\% silt and kaolinite clay matrix; basal $0.3 \mathrm{ft}$ contains abundant subangular to subround quartz granules and pebbles up to $1 \mathrm{~cm}$ in diameter; no transmissivity; light-greenish-gray (5G 8/1) mottled dark yellowish orange (10YR 6/6). Box 40.

432.5 - $435.2 \mathrm{ft}$ : SILT, very clayey, grading down to SILT, clayey, contains 2-3\% vf quartz sand; silvery mica (tr); blocky ped and root structures; greenish gray $(5 G 6 / 1)$ mottled and streaked moderate reddish brown $(10 R 4 / 6)$, dark reddish brown $(10 R 3 / 4)$, and moderate red (5R 5/4). Box 40 and Box 41. 
$435.2-440.0 \mathrm{ft}:$ No recovery.

\section{0 - 450.0 ft: Run 58}

440.0 - 442.9 ft: SILT, clayey, contains 2-3\% vf quartz sandy; silvery mica (tr); blocky ped and root structures; greenish gray $(5 G 6 / 1)$ mottled and streaked moderate reddish brown $(10 R$ $4 / 6)$, dark reddish brown ( $10 R 3 / 4)$, and moderate red (5R 5/4). Box 41.

442.9 - $448.7 \mathrm{ft}$ : SAND, quartz, vf-f grading down to vf-m, subangular to subround, with 10-15\% silt and clay matrix; $2 \%$ silvery mica, 1-2\% sand-size black lignite, and scattered grains of fine round black phosphate disseminated throughout; banded to blotched color texture, greenish gray $(5 G 6 / 1)$ mottled and streaked dark reddish brown $(10 R 3 / 4)$ and moderate yellowish brown (10YR 5/4). Box 41 and Box 42.

$448.7-450.0 \mathrm{ft}$ : No recovery.

\section{0 - 460.0 ft: Run 59}

450.0 - $456.2 \mathrm{ft}$ : SAND, quartz, vf-m, subround grading down to vf-c, subangular, very poorly sorted, with $10-15 \%$ silt and clay matrix; $2-3 \%$ disseminated lignite, silvery mica (tr); granules present below $451.3 \mathrm{ft}$ and pebbles present below $453.0 \mathrm{ft}$, mostly quartz but some feldspar, subangular to subround; silt and clay content increases in basal $0.3 \mathrm{ft}$; texture massive; greenish gray (5G 6/1) mottled and streaked grayish red $(10 R 4 / 2)$, dusky red (5R 3/4), and dark yellowish orange (10YR 6/6). Box 42 and Box 43.

$456.2-458.6 \mathrm{ft}$ : SILT, very clayey, grading down by $457.9 \mathrm{ft}$ to SILT, clayey, contains $\sim 10 \% \mathrm{vf}-\mathrm{f}$ subround quartz sand; $\sim 1 \%$ silvery mica; greenish gray $(5 G 6 / 1)$ streaked grayish red $(10 R 4 / 2)$, dark yellowish orange (10YR 6/6), and light olive (10Y 5/4). Box 43.

458.6 - $460.0 \mathrm{ft}$ : No recovery.

\section{0 - 470.0 ft: Run 60}

460.0 - 464.2 ft: SILT, clayey, contains $~ 10 \%$ vf-f subround quartz sand; $~ 1 \%$ silvery mica; greenish gray $(5 G 6 / 1)$ streaked grayish red (10R $4 / 2)$, dark yellowish orange (10YR 6/6), and light olive (10Y 5/4). Box 43 and Box 44.

464.2 - $464.5 \mathrm{ft}$ : Sand, quartz, vf-f and sparse $\mathrm{m}$, subround, with 5-10\% silt and clay matrix; 1\% finely disseminated fine lignite, silvery mica (tr); texture massive; between yellowishgray and light olive gray (5Y 7/1). Box 44.

464.5 - 464.7 ft: SILT, clayey, contains $\sim 10 \%$ vf-f subround quartz sand; $~ 1 \%$ silvery mica; greenish gray $(5 G 6 / 1)$ streaked grayish red (10R 4/2), dark yellowish orange (10YR 6/6), and light olive (10Y 5/4). Box 44.

464.7 - $464.8 \mathrm{ft}$ : Sand, quartz, vf-f and sparse m, subround, with 5-10\% silt and clay matrix; 1\% finely disseminated fine lignite, silvery mica (tr); texture massive; between yellowish gray and light olive gray (5Y 7/1). Box 44.

\section{Cape Fear Formation (marine facies) ( = Pleasant Creek Formation?)}

$464.8-469.1 \mathrm{ft}:$ SAND, quartz, mostly f-m but locally f-c, subangular, with $\sim 30 \%$ silt and clay matrix; $\sim 1 \%$ fine pyrite balls and fine lignite, silvery mica (tr); greenish gray (6GY 6/1) to dusky yellow green $(5 G Y 5 / 2)$. Box 44.

$469.1-470.0 \mathrm{ft}$ : No recovery. 


\section{0 - 480.0 ft: Run 61}

470.0 - $472.8 \mathrm{ft}$ : SAND, quartz, vf-f, subangular to subround, ranging up to $\mathrm{m}$-vc, subangular, with $\sim 10 \%$ (coarser fraction) to $20 \%$ (finer fraction) silt and clay matrix; silvery mica (tr); local lenses have subangular quartz granules and pebbles to $0.5 \mathrm{~cm}$ and mudballs 3 to $5 \mathrm{~cm}$ in diameter; semilithified; greenish gray $(5 G 6 / 1)$ with streaks of light olive brown (5Y 5/6) grading downward to light olive gray $(5 Y 6 / 1)$. Box 44.

$472.8-480.0 \mathrm{ft}$ : No recovery.

\section{0 - 490.0 ft: Run 62}

480.0 - $482.1 \mathrm{ft}:$ SAND, quartz, f-vc, subangular, with 40-50\% silt and clay matrix; scattered subangular quartz granules present; clayball at top; texture massive; light olive gray $(5 Y 6 / 1)$ grading at about 481.4 to greenish gray $5 G Y 6 / 1)$. Box 45.

482.1 - $482.2 \mathrm{ft}$ : SAND, quartz, m-vc, angular; angular quartz granules abundant; basal contact sharp; pale reddish brown (10R 5/4) and moderate yellowish brown (10YR 5/4). Box 45.

\section{Unnamed Formation ( = "Cape Fear Formation" of South Carolina usage)}

482.2 - $484.6 \mathrm{ft}$ : SILT, very clayey, contains 2-3\% vf quartz sand; blocky ped structures and root ghosts present throughout; varicolored blotches and streaks of dark reddish brown (10R 3/4), moderate brown (5YR 4/4), light olive gray $(5 Y 6 / 1)$, light greenish gray $(5 G Y 8 / 1)$, and dark yellowish orange (10YR 6/6). Box 45.

484.6 - $490.0 \mathrm{ft}:$ No recovery.

\section{0 - 500.0 ft: Run 63}

490.0 - $499.3 \mathrm{ft}$ : SILT, very clayey, contains 2-3\% vf quartz sand; blocky ped structures and root ghosts present throughout; slickensides scattered throughout interval; varicolored blotches and streaks of dark reddish brown (10R 3/4), moderate brown (5YR 4/4), light olive gray (5Y 6/1), light-greenish-gray (5GY 8/1), and dark-yellowish-orange (10YR 6/6), grading down below $497.6 \mathrm{ft}$ to olive gray (5Y 4/1). Box 45 and Box 46.

499.3 - $500.0 \mathrm{ft}:$ No recovery.

\section{0 - 510.0 ft: Run 64}

500.0 - 508.9 ft: SILT, very clayey, contains 2-3\% vf quartz sand; blocky ped structures and root ghosts present throughout; slickensides abundant throughout interval; olive gray ( $5 Y$ 4/1) sparsely mottled dusky red (10R 2/2) and moderate yellowish brown (10YR 5/4). Box 46 and Box 47.

508.9 - $509.1 \mathrm{ft}$ : BRECCIA, angular, clasts composed of clayey silt; matrix olive gray (5Y 4/1), clasts greenish gray $(5 G Y 6 / 1)$. Box 47 and Box 48.

$509.1-510.0 \mathrm{ft}$ : SILT, very clayey, contains 2-3\% vf quartz sand; ped structures and root casts abundant, texture anastomosing; iron nodules at base; greenish gray $(5 G Y 6 / 1)$ mottled moderate brown (5YR 4/4) and dark reddish brown (10R 3/4). Box 48. 


\section{0 - 520.0 ft: Run 65}

510.0 - $515.6 \mathrm{ft}:$ SILT, very clayey, contains sparse grains of quartz sand below $513.0 \mathrm{ft} ; \sim 1 \%$ grading down to $\sim 3 \%$ silvery mica; root structures abundant down to 515.0, then bedded below that; greenish gray $(5 G Y 6 / 1)$ mottled moderate brown $(5 Y R 4 / 4)$ and dark reddish brown $(10 R 3 / 4)$. Box 48.

515.6 - $518.5 \mathrm{ft}$ : SAND, quartz, vf-f coarsening downward to vf-m, subangular to subround, with $\sim 30 \%$ silt and clay matrix; up to $5 \%$ silvery mica, black lignite grains $\sim 1-2 \%$ at top but increase downward in abundance to $20-25 \%$, phlogopite (tr); basal $0.3 \mathrm{ft}$ has quartz, phosphate, and pyrite granules, and a topaz(?) clast; greenish gray $(5 G Y 6 / 1)$ grading down to dark greenish gray $(5 G Y 4 / 1)$ mottled and streaked moderate yellowish brown (10YR 4/2) and moderate brown (5YR 4/4). Box 48 and Box 49.

$518.5-520.0 \mathrm{ft}$ : No recovery. High values on gamma $\log$ in this interval suggest interval is phosphate-rich.

\section{0 - 530.0 ft: Run 66}

520.0 - $523.6 \mathrm{ft}:$ SAND, quartz, vf-f, subround, with 10-15\% silt and clay matrix; $10 \%$ chlorite, grains up to medium-size; clayballs present below $522.5 \mathrm{ft}$; lense of angular ilmenite(?) sand at $522.7 \mathrm{ft}$; gradational with bed below; grayish green (10GY 5/2) mottled moderate olive brown ( $5 Y 4 / 4)$ and moderate brown (5YR 3/4), clayballs dark greenish gray $(5 G Y 4 / 1)$. Box 49.

523.6 - $526.2 \mathrm{ft}$ : SAND, quartz, f-vc, with scattered subangular granules and $\sim 10 \%$ silt and clay matrix; $1 \%$ ilmenite; dusky yellow green (5GY 5/2) mottled moderate brown (5YR $4 / 4)$ and light olive (10Y 5/4), clayballs dark greenish gray (5GY 4/1). Box 49 and Box 50.

$526.2-530.0 \mathrm{ft}$ : No recovery. High values on gamma log in this interval suggest interval is phosphate-rich.

\section{0 - 540.0 ft: Run 67}

530.0 - $530.7 \mathrm{ft}$ : SAND, quartz, f-vc, angular to subangular, subangular to subround quartz granules and pebbles up to $1.5 \mathrm{~cm}$ in diameter abundant; texture massive; sharp basal contact; greenish gray $(5 G Y 6 / 1)$. Box 50 .

530.7 - $531.2 \mathrm{ft}$ : SILT, very clayey; gradational with bed below; grayish black $(N 2)$. Box 50 .

531.2 - $534.2 \mathrm{ft}$ : SILT, clayey, contains $\sim 10 \%$ vf-f subround grading down to $20-30 \%$ f-m subround quartz sand; faint root structures visible; light olive gray $(5 Y 6 / 1)$. Box 50.

534.2 - $540.0 \mathrm{ft}$ : No recovery, drilled like sand.

\section{0 - 550.0 ft: Run 68}

540.0 - $543.9 \mathrm{ft}:$ SAND, quartz, f-m, subangular, with $~ 10 \%$ silt and clay matrix, interbedded with SAND, f-vc, subangular, with 7-10\% silt and clay matrix, coarser fraction contains subangular to subround quartz granules and rare subangular quartz pebbles up to $1 \mathrm{~cm}$ in diameter; up to $2 \%$ silvery mica locally; faintly bedded, bedding planes defined by silvery mica flakes; greenish gray $(5 G 6 / 1)$ mottled grayish green $(10 G Y 5 / 2)$, light 
olive gray (5Y 6/1), moderate reddish brown (10R 4/6), and grayish red (10R 4/2). Box 50 and Box 51.

543.9 - $550.0 \mathrm{ft}$ : No recovery.

\section{0 - 560.0 ft: Run 69}

550.0 - 553.1 ft: SAND, quartz, f-c, subangular, with 10-15\% silt and clay matrix; $1 \%$ silvery mica and chlorite, some mica grains up to vc-size; interbedded with GRANULES, quartz, with $\mathrm{f}-\mathrm{vc}$ subangular to subround quartz sand and $\sim 7 \%$ silt and clay matrix; $\sim 2 \%$ silvery mica; contains subangular quartz pebbles up to $2 \mathrm{~cm}$ in diameter, flattened clayballs present locally; granule beds $\sim 0.1 \mathrm{ft}$ thick; faintly bedded; low porosity, low transmissivity, matrix moderately tight; grayish green $(10 G Y 5 / 2)$ mottled dusky yellow green $(5 G Y 5 / 2)$. Box 51 .

$553.1-560.0 \mathrm{ft}$ : No recovery.

\section{0 - 570.0 ft: Run 70}

560.0 - $562.0 \mathrm{ft}:$ SAND, quartz, vf-vc, subangular, contains scattered subangular granules and $<5 \%$ silt and clay matrix; $2 \%$ silvery mica; scattered small clayballs present; massive texture; sharp basal contact; moderate porosity, medium transmissivity; pale yellowish brown (10YR 6/2), color possibly influenced by drilling mud. Box 51.

562.0 - $562.4 \mathrm{ft}$ : SAND, quartz, vf-m, subround, with 10-15\% silt and clay matrix; $2 \%$ silvery mica; yellowish gray (5Y7/2). Box 51 .

$562.4-570.0 \mathrm{ft}$ : No recovery.

\section{0 - 580.0 ft: Run 71}

$570.0-571.8 \mathrm{ft}:$ SAND, quartz, f-m, subround, with $\sim 10 \%$ silt and clay matrix, locally $3-5 \%$ silvery mica, up to vc-size; chlorite (tr), $\sim 1 \%$ organics, ilmenite lenses near base; faintly bedded; sharp basal contact; greenish gray (5GY 6/1). Box 51 and Box 52.

\section{Clubhouse Formation}

571.8 - $572.5 \mathrm{ft}$ : SILT, very clayey, contains $\sim 1 \%$ vf-f rounded quartz sand; thinly laminated; small burrows present, possibly crayfish; grayish black $(N 2)$ grading down to dark gray $(N$ 3 ) and then back to grayish black. Box 52.

572.5 - $572.7 \mathrm{ft}$ : SILT, clayey, contains 3-4\% vf-f subangular to subround quartz sand; silvery mica (tr); disturbed bedding and small clayballs or filled burrows up to $0.5 \mathrm{~cm}$ in diameter present; sharp but irregular basal contact; olive black (5Y 2/1), clayballs grayish black (N 2). Box 52.

572.7 - $574.0 \mathrm{ft}$ : SILT, clayey, contains $\sim 5 \%$ vf-f subround quartz sand; silvery mica (tr), mediumgrained detrital pyrite (tr); massive except for root structures and occasional burrows; rapidly gradational with bed below; olive gray ( $5 Y 4 / 1)$ mottled dusky green $(5 G 3 / 2)$, grayish black $(N 2)$ (root structures), and (near base only) grayish olive green (5GY 3/2). Box 52. 
574.0 - $576.8 \mathrm{ft}:$ SILT, slightly clayey, contains $\sim 30 \%$ vf-f subround to round quartz sand; $\sim 1 \%$ ilmenite and $\sim 1 \%$ silvery mica; texture massive; gradational with bed below; olive gray $(5 Y 4 / 1)$. Box 52.

$576.8-579.8 \mathrm{ft}$ : SAND, quartz, vf-m, subround to round, with 15-20\% silt and clay matrix decreasing downward to $\sim 5 \%$ silt and clay; $\sim 3 \%$ fine ilmenite, $\sim 3 \%$ silvery mica (up to vc-size) increasing downward to $10-15 \%$ silvery mica locally; clay-lined crayfish(?) burrows present; faintly bedded and locally cross-bedded; dark greenish gray (5GY 4/1), burrows olive gray (5Y 4/1). Box 52 and Box 53.

$579.8-580.0 \mathrm{ft}:$ No recovery.

\section{0 - 583.0 ft: Run 72}

580.0 - 580.3 ft: SAND, quartz, vf-m, subround to round, with $<5 \%$ silt and clay matrix; $~ 3 \%$ fine ilmenite, $10-15 \%$ silvery mica up to vc-size; clay-lined crayfish(?) burrows present; faintly bedded and locally cross-bedded; dark greenish gray $(5 G Y 4 / 1)$, burrows olive gray $(5 Y 4 / 1)$. Box 53.

580.3 - $582.5 \mathrm{ft}$ : SAND, quartz, f grading down to f-m, subround, with 10-15\% silt and clay matrix; 5$10 \%$ silvery mica, $\sim 3 \%$ dark heavy minerals, $\sim 1 \%$ apatite, phlogopite (tr); massive texture; soft; dark greenish gray $(5 G Y 4 / 1)$. Box 53.

$582.5-583.0 \mathrm{ft}$ : No recovery.

\section{0 - 590.0 ft: Run 73}

583.0 - 590.0 ft: SAND, quartz, vf-f, subround to round, with 3\% or less silt and clay matrix; $\sim 5 \%$ silvery mica and chlorite grading down to $\sim 20 \%$ near base and up to vc-size; $\sim 10 \%$ lignite chips but locally more; apatite? (tr), phlogopite (tr), a few stringers of dark heavy minerals (illite?, magnetite?); faintly bedded; dark greenish gray (5GY 4/1). Box 53 and Box 54.

\section{0 - 600.0 ft: Run 74}

590.0 - $593.1 \mathrm{ft}$ : SAND, quartz, vf-f, subround to round, grading down to f-c, subangular to subround, with $3 \%$ or less silt and clay matrix; $\sim 20 \%$ silvery mica up to vc-size; $\sim 10 \%$ lignite chips but locally more; apatite? (tr), phlogopite (tr), a few stringers of dark heavy minerals (illite?, magnetite?); faintly bedded; dark greenish gray (5GY4/1). Box 54 .

593.1 - $597.7 \mathrm{ft}$ : SAND, quartz, vf-f, subangular to subround, with 3-5\% silt and clay matrix; $\sim 3 \% \mathrm{f}-\mathrm{m}$ silvery mica and chlorite, grading down to $\sim 20 \%$ by base with mica flakes ranging up to granule-size; $\sim 3 \%$ dark organics and dark heavy minerals; $\sim 1 \%$ phlogopite; massive texture, soft; high porosity, medium to high transmissivity; dark greenish gray $(5 G Y 4 / 1)$. Box 54 and Box 55.

597.7 - $597.8 \mathrm{ft}$ : GNEISS, biotite and silvery mica; strongly foliated; greenish black (5G 2/1). Box 55.

597.8 - $597.9 \mathrm{ft}$ : QUARTZITE, f-c, subangular, silica cemented; massive; this and interval before very slow drilling, probable erratic cobble; olive gray $(5 Y 4 / 1)$. Box 55.

597.9 - $599.2 \mathrm{ft}$ : SAND, quartz, vf-f, subangular to subround, with 3-5\% silt and clay matrix; $20 \% \mathrm{f}-$ $\mathrm{m}$ silvery mica and chlorite with mica flakes ranging up to granule-size; $\sim 3 \%$ dark organics and dark heavy minerals; $\sim 1 \%$ phlogopite; massive texture; soft; high porosity, medium to high transmissivity; dark greenish gray $(5 G Y 4 / 1)$. Box 55 .

$599.2-600.0 \mathrm{ft}$ : No recovery. 


\section{0 - 610.0 ft: Run 75}

600.0 - 600.5 ft: SAND, quartz, vf-f, subangular to subround, with 3-5\% silt and clay matrix; 20\% f$\mathrm{m}$ silvery mica and chlorite with mica flakes ranging up to granule-size; $\sim 3 \%$ dark organics and dark heavy minerals; $1 \%$ phlogopite; faintly bedded, soft; high porosity, medium to high transmissivity; dark greenish gray $(5 G Y 4 / 1)$. Box 55.

$600.5-600.6 \mathrm{ft}$ : LIGNITE, locally up to $20 \%$ silvery mica and chlorite; possibly a flattened log or large branch; black (N 1). Box 55.

$600.6-603.2 \mathrm{ft}$ : SAND, quartz, vf-m, subround, with $3 \%$ or less silt and clay matrix; $1 \%$ disseminated dark organics, silvery mica (tr); clay stringers and clayballs up to $4 \mathrm{~cm}$ long scattered about; rare chunks of lignite up to $2 \mathrm{~cm}$ present; faintly bedded; dark greenish gray $(5 G Y 4 / 1)$, clayballs olive black (5Y2/1). Box 55.

$603.2-604.9 \mathrm{ft}$ : SAND, quartz, vf-f, subround, with $3 \%$ or less silt and clay matrix; $\sim 2 \%$ disseminated dark organics, $\sim 1 \%$ silvery mica; very faintly bedded, dark greenish gray $(5 G Y 4 / 1)$. Box 55 and Box 56.

$604.9-609.0 \mathrm{ft}$ : SAND, quartz, vf-f, subround, with 3\% or less silt and clay matrix; $2 \%$ disseminated dark organics, $\sim 1 \%$ silvery mica; very faintly bedded, abundant mudballs scattered about; porosity low, transmissivity moderate; dark greenish gray $(5 G Y 4 / 1)$, mudballs greenish black (5GY 2/1). Box 56.

$609.0-610.0 \mathrm{ft}$ : No recovery.

\section{0 - 620.0 ft: Run 76}

$610.0-610.1 \mathrm{ft}:$ SAND, quartz, vf-f, subround, with 3\% or less silt and clay matrix; $2 \%$ disseminated dark organics, $\sim 1 \%$ silvery mica; very faintly bedded; dark greenish gray $(5 G Y 4 / 1)$. Box 56.

610.1 - 610.4 ft: SILT, clayey, $2 \%$ f quartz sand; massive texture; olive black (5Y2/1). Box 56.

$610.4-612.4 \mathrm{ft}$ : SAND, quartz, vf, with 3\% or less silt and clay matrix; $2 \%$ disseminated fine lignite, $\sim 5 \%$ chlorite up to medium-size, $20 \%$ or less silvery mica up to coarse-size; massive; dark greenish gray $(5 G 4 / 1)$. Box 56 .

$612.4-615.8 \mathrm{ft}$ : SILT, clayey, contains $10-15 \%$ vf quartz sand; $15 \%$ or less silvery mica up to medium-size; dark greenish gray $(5 G 4 / 1)$; interbedded with SILT, very clayey, sticky and gummy; grayish black $(N 2)$ grading down to greenish black $(5 G Y 2 / 1)$ at base; thinly bedded near base, lignite-sand defines bedding planes; slight porosity, no transmissivity. Box 56 and Box 57.

615.8 - 619.5 ft: SAND, quartz, vf-f, subround, with $\sim 7 \%$ silt and clay matrix; $\sim 10 \%$ disseminated lignite sand, $\sim 2 \%$ silvery mica, $\sim 1 \%$ chlorite; massive texture except for a few clayrich stringers; dark greenish gray $(5 G 4 / 1)$, clay-rich stringers medium dark gray $(N$ 4). Box 57.

$619.5-620.0 \mathrm{ft}:$ No recovery. 
620.0 - 621.0 ft: SAND, quartz, vf, with 3-5\% silt and clay matrix; 5\% silvery mica, $2 \%$ chlorite; sparsely shelly, subround quartz granules at base; dark greenish gray $(5 G Y 4 / 1)$. Box 57 and Box 58.

$621.0-624.8 \mathrm{ft}:$ SAND, quartz, vf, with 5-7\% silt and clay matrix; $10 \%$ increasing downward to $\sim 20 \%$ silvery mica, $5-7 \%$ grading down to $\sim 15 \%$ chlorite, phlogopite (tr); dark greenish gray $(5 G Y 4 / 1)$. Box 58 .

$624.8-625.2 \mathrm{ft}$ : SILT, clayey, contains $\sim 3 \%$ vf quartz sand; massive texture; dry; dark-gray $(N 3)$. Box 58.

$625.2-625.6 \mathrm{ft}$ : SILT, slightly clayey, contains $10-15 \%$ vf quartz sand; $~ 15 \% \mathrm{f}-\mathrm{m}$ silvery mica and chlorite; massive texture; dark greenish gray $(5 G Y 4 / 1)$. Box 58 .

$625.6-629.0 \mathrm{ft}$ : SAND, quartz, vf, with $\sim 15 \%$ silt and clay matrix; $8-10 \%$ chlorite, $\sim 5 \%$ silvery mica; banded in top $0.2 \mathrm{ft}$ then massive texture below that; dark greenish gray (5GY 4/1). Box 58 and Box 59 .

$629.0-630.0 \mathrm{ft}$ : No recovery.

\section{0 - 640.0 ft: Run 78}

$630.0-630.5 \mathrm{ft}:$ SAND, quartz, vf, with $\sim 15 \%$ silt and clay matrix; $8-10 \%$ chlorite, $\sim 5 \%$ silvery mica; massive texture; dark greenish gray (5GY 4/1). Box 59 .

$630.5-631.4 \mathrm{ft}$ : SAND, quartz, vf, with $\sim 10 \%$ silt and clay matrix; $\sim 1 \%$ silvery mica, $\sim 1 \%$ chlorite; massive texture; greenish gray $(5 G Y 6 / 1)$. Box 59 .

$631.4-640.0 \mathrm{ft}$ : No recovery.

\section{0 - 646.5 ft: Run 79}

640.0 - 640.4 ft: SILT, very clayey, contains sparse quartz sand grains; faintly layered; greenish black (5GY2/1). Box 59.

$640.4-641.7 \mathrm{ft}$ : SILT, clayey, contains $\sim 30 \%$ vf quartz sand; $\sim 2 \%$ silvery mica; faintly bedded; dark greenish gray (5GY 4/1); interbedded with SILT, very clayey; slightly calcareous; greenish black (5GY 2/1); individual layers up to $0.2 \mathrm{ft}$ thick. Box 59 .

$641.7-645.5 \mathrm{ft}$ : SAND, quartz, vf, with $\sim 15 \%$ silt and clay matrix; $\sim 1 \%$ silvery mica, chlorite (tr); massive texture; high transmissivity; dark greenish gray (5GY 4/1). Box 59 .

$645.5-646.2 \mathrm{ft}$ : SILT, clayey, contains $\sim 30 \%$ vf quartz sand; $\sim 2 \%$ silvery mica; faintly bedded; dark greenish gray $(5 G Y 4 / 1)$; interbedded with SILT, very clayey; slightly calcareous; greenish black (5GY 2/1); individual layers up to $0.2 \mathrm{ft}$ thick. Box 59 and Box 60 .

$646.2-646.5 \mathrm{ft}$ : LIMESTONE, micrite; $\sim 5 \%$ silvery mica, $\sim 5 \%$ chlorite; small wood chips present; contains molds and casts of pelecypod shells; thinly bedded; calcite-filled fractures present that narrow upward; medium dark gray $(N 4)$. Box 60.

\section{5 - 650.0 ft: Run 80}

646.5 - 646.6 ft: LIMESTONE, micrite; 5\% silvery mica, 5\% chlorite; small wood chips present; contains molds and casts of pelecypod shells; thinly bedded; medium dark gray $(N 4)$. Box 60.

$646.6-649.8 \mathrm{ft}:$ SAND, quartz, vf, with $\sim 15 \%$ silt and clay matrix; $~ 1 \%$ silvery mica, chlorite (tr), calcareous; thin bedded grading down to thicker bedded, bedding defined by percent lime content; dark greenish gray (5GY 4/1). Box 60. 
$649.8-650.0 \mathrm{ft}:$ No recovery.

\section{0 - 654.3 ft: Run 81}

$650.0-654.2 \mathrm{ft}:$ SAND, vf, with $\sim 15 \%$ silt and clay matrix; $\sim 5 \%$ dark heavy minerals, $\sim 3 \%$ chlorite, $\sim 1 \%$ silvery mica, faintly calcareous; molds and casts of pelecypods locally present and some rotten shells; massive texture; drilling mud injection parallel to bedding at centimeter or less intervals; grayish green (5G 5/2). Box 60 and Box 61.

654.2 - $654.3 \mathrm{ft}$ : SANDSTONE, vf, with $\sim 15 \%$ silt and clay matrix; dolomite-cemented; $\sim 5 \%$ dark heavy minerals, $\sim 3 \%$ chlorite, $\sim 1 \%$ silvery mica; massive texture; grayish green $(5 G$ 5/2). Box 61.

\section{3 - 660 ft: Run 82}

654.3 - $654.9 \mathrm{ft}$ : SANDSTONE, vf, with $\sim 15 \%$ silt and clay matrix; dolomite-cemented; $\sim 5 \%$ dark heavy minerals, $\sim 3 \%$ chlorite, $\sim 1 \%$ silvery mica; massive texture; grayish green $(5 G$ 5/2). Box 61.

654.9 - $657.7 \mathrm{ft}:$ SAND, quartz, vf, with $\sim 15 \%$ silt and clay matrix; $\sim 5 \%$ chlorite, $\sim 3 \%$ glauconite(?) and dark heavy minerals, $\sim 1 \%$ silvery mica, calcareous; very thin interbeds of more clay-rich sand and sparse shells present; coarsens downward and very shelly near base; dark greenish gray $(5 G Y 4 / 1)$. Box 61.

$657.7-658.2 \mathrm{ft}$ : SILT, very clayey, contains <5\% vf quartz sand; sticky, noncalcareous; thinly laminated; olive black (5Y 2/1). Box 61.

658.2 - $659.3 \mathrm{ft}$ : SAND, quartz, vf, with $\sim 15 \%$ silt and clay matrix; 7-8\% silvery mica, $\sim 5 \%$ chlorite, $\sim 3 \%$ glauconite(?) and dark heavy minerals, calcareous; abundant shell fragments; massive texture; coarsens downward and very shelly near base; dark greenish gray (5GY 4/1). Box 61.

659.3 - $660.0 \mathrm{ft}$ : COQUINA, indurated, with quartz-calcite sand matrix, greenish gray (5GY 6/1). Box 61.

\section{0 - 670.0 ft: Run 83}

$660.0-669.3 \mathrm{ft}:$ SAND, quartz, vf-f, with $\sim 5 \%$ or less silt and clay matrix, slightly calcareous; $5-10 \%$ silvery mica; shelly, mostly pelecypods, some with nacreous luster; faintly bedded, bedding defined by clayey intervals; scattered burrows, not clay-lined; dark greenish gray $(5 G$ 4/1). Box 61, Box 62 and Box 63.

$669.3-670.0 \mathrm{ft}:$ SAND, quartz, vf-f, with $\sim 5 \%$ or less silt and clay matrix, slightly calcareous; $5-10 \%$ silvery mica; dark greenish gray $(5 G 4 / 1)$. Box 63.

\section{0 - 678.5 ft: Run 84}

670.0 - 672.9 ft: SAND, quartz, vf, well sorted, with 5\% silt and clay matrix; 5\% silvery mica; sparsely fossiliferous, mostly pelecypods; faint bedding; dark greenish gray ( $5 G 4 / 1)$. Box 63. 
672.9 - 674.4 ft: SILT, clayey, contains vf quartz sand; moderately abundant pelecypod shells; fragments of lignite and pyritized lignite up to $10 \mathrm{~mm}$ in length; faint bedding; dark greenish gray $(5 G 4 / 1)$. Box 63.

$674.4-675.9 \mathrm{ft}$ : SAND, quartz, vf, well sorted, with $\sim 5 \%$ silt and clay matrix; $~ 5 \%$ silvery mica; sparsely fossiliferous, mostly pelecypods; faint bedding; dark greenish gray $(5 G 4 / 1)$. Box 63 and Box 64.

675.9 - 676.1 ft: SAND, quartz, vf, with clay matrix; 10\% silvery mica; abundant pelecypod shells, abundant fragments of lignite and pyritized lignite up to $10 \mathrm{~mm}$ in length; dark greenish gray $(5 G 4 / 1)$. Box 64 .

$676.1-678.3 \mathrm{ft}$ : SAND, quartz, vf, well sorted, with $\sim 5 \%$ silt and clay matrix; $~ 5 \%$ silvery mica; sparsely fossiliferous, mostly pelecypods; faint bedding, horizontal to slightly inclined ( $5^{\circ}$ or less); dark greenish gray $(5 G 4 / 1)$. Box 64 .

$678.3-678.5 \mathrm{ft}$ : No recovery.

\section{5 - 679.7 ft: Run 85}

678.5 - 679.4 ft: COQUINA, with vf-f quartz-calcite sand matrix, calcite-cemented; large pelecypod shells abundant, abundant lignite clasts up to $14 \mathrm{~mm}$ in diameter; dark greenish gray $(5 G 4 / 1)$. Box 64 .

$679.4-679.7 \mathrm{ft}:$ No recovery.

\section{7 - 690.0 ft: Run 86}

679.7 - $679.8 \mathrm{ft}$ : COQUINA, with vf-f quartz-calcite sand matrix, calcite-cemented; large pelecypod shells abundant, abundant lignite clasts up to $14 \mathrm{~mm}$ in diameter; dark greenish gray $(5 G 4 / 1)$. Box 64 .

679.8 - $680.8 \mathrm{ft}$ : SAND, quartz, vf, well sorted, with $5 \%$ silt and clay matrix; $5 \%$ silvery mica; sparsely fossiliferous, mostly pelecypods; faint bedding; dark greenish gray $(5 G 4 / 1)$. Box 64 .

$680.8-684.7 \mathrm{ft}$ : SAND, quartz, vf-f, well sorted, with $\sim 10 \%$ silt and clay matrix; $~ 5 \%$ silvery mica, chlorite/ilmenite (tr); 10\% shell material, mostly pelecypod; bedding horizontal; greenish black $(5 G 2 / 1)$. Box 64 and Box 65 .

$684.7-687.0 \mathrm{ft}$ : SAND, quartz, vf-f, well sorted, with $\sim 5 \%$ silt and clay matrix; $10 \%$ silvery mica, 1 $2 \%$ fine sand-size lignite, chlorite-ilmenite (tr); $\sim 10 \%$ shell material, mostly pelecypod; bedding horizontal; dark greenish gray $(5 G 4 / 1)$. Box 65 .

687.0 - 688.7 ft: SAND, quartz, vf-f, well sorted, with $\sim 10 \%$ silt and clay matrix; $\sim 5 \%$ silvery mica, chlorite/ilmenite (tr); $10 \%$ shell material, mostly pelecypod; bedding horizontal; greenish black $(5 G 2 / 1)$. Box 65 .

$688.7-689.5 \mathrm{ft}$ : SAND, quartz, vf-f, well sorted, with $\sim 5 \%$ silt and clay matrix; $~ 10 \%$ silvery mica, 1$2 \%$ fine sand-size lignite, chlorite-ilmenite (tr); $\sim 10 \%$ shell material, mostly pelecypod; bedding horizontal; dark greenish gray $(5 G 4 / 1)$. Box 65 .

$689.5-690.0 \mathrm{ft}$ : SAND, quartz, vf-f, well sorted, with $\sim 10 \%$ silt and clay matrix; $~ 5 \%$ silvery mica, chlorite/ilmenite (tr); 10\% shell material, mostly pelecypod; bedding horizontal; greenish black $(5 G 2 / 1)$. Box 65 . 


\section{0 - 700.0 ft: Run 87}

690.0 - $691.9 \mathrm{ft}:$ SAND, quartz, vf-f, well sorted, with $\sim 10 \%$ silt and clay matrix; $~ 5 \%$ silvery mica, chlorite/ilmenite (tr); 10\% shell material, mostly pelecypod; thin lignite layer at $691.4 \mathrm{ft}$; bedding horizontal; greenish black (5G 2/1). Box 65 and Box 66.

691.9 - $692.9 \mathrm{ft}$ : COQUINA, mostly pelecypods, with fine quartz-calcite matrix, calcite-cemented; two $4 \mathrm{~mm}$ phosphate pebbles present at $692.5 \mathrm{ft}$; dark greenish gray $(5 G 4 / 1)$. Box 66 .

692.9 - $696.5 \mathrm{ft}$ : SAND, quartz, vf-f, well sorted, with $\sim 10 \%$ silt and clay matrix; $\sim 5 \%$ silvery mica, chlorite/ilmenite (tr); $10 \%$ shell material, mostly pelecypod; bedding mostly horizontal but wavy or distorted around $695.0 \mathrm{ft}$; greenish black $(5 G 2 / 1)$. Box 66.

696.5 - 698.1 ft: SAND, quartz, vf-f, well sorted, with $\sim 5 \%$ silt and clay matrix; $10 \%$ silvery mica, 1 $2 \%$ fine sand-size lignite, chlorite-ilmenite (tr), orange-pink heavy minerals (tr); $\sim 10 \%$ shell material, mostly pelecypod; bedding horizontal; dark greenish gray ( $5 \mathrm{G}$ 4/1). Box 66.

698.1 - $700.0 \mathrm{ft}:$ SAND, quartz, vf-f, well sorted, with $\sim 10 \%$ silt and clay matrix; $~ 5 \%$ silvery mica, chlorite/ilmenite (tr); 10\% shell material, mostly pelecypod; bedding horizontal; greenish black (5G 2/1). Box 66 and Box 67.

\section{0 - 710.0 ft: Run 88}

700.0 - $709.6 \mathrm{ft}:$ SAND, quartz, vf-f, with 5-15\% silt and clay matrix; 5-8\% silvery mica, chlorite/ilmenite (tr), glauconite (tr), colored heavy minerals (tr); sparse shells, mostly pelecypods, one ammonite at $708.4 \mathrm{ft}$; horizontal bedding, beds 0.5 to $1.5 \mathrm{ft}$ thick; dark greenish gray $(5 G$ 4/1). Box 67 and Box 68 .

709.6 - $710.0 \mathrm{ft}$ : No recovery.

\section{0 - 712.0 ft: Run 89}

710.0 - $711.5 \mathrm{ft}:$ SAND, quartz, vf-f, with 5-15\% silt and clay matrix; 5-8\% silvery mica, chlorite/ilmenite (tr), glauconite (tr), colored heavy minerals (tr); sparse shells, mostly pelecypods; horizontal bedding, beds 0.5 to $1.5 \mathrm{ft}$ thick; dark greenish gray $(5 G 4 / 1)$. Box 68.

711.5 - $712.0 \mathrm{ft}$ : COQUINA, mostly pelecypods, with $\sim 20 \%$ carbonate matrix; dark greenish gray $(5 G$ 4/1). Box 68.

\section{0 - 720.0 ft: Run 90}

712.0 - $712.7 \mathrm{ft}$ : COQUINA, mostly pelecypods, with $\sim 20 \%$ carbonate matrix; dark greenish gray $(5 G$ 4/1). Box 68.

712.7 - $717.8 \mathrm{ft}$ : SAND, quartz, vf-f, well sorted, with 10-15\% silt and clay matrix, calcareous; 5-10\% silvery mica, chlorite (tr), glauconite (tr), colored heavy minerals (tr); abundant fossils, mostly pelecypods, some with nacreous luster; faint thin to wavy bedding; dark greenish gray (5G 4/1). Box 68 and Box 69.

$717.8-720.0 \mathrm{ft}:$ SAND, quartz, vf-f, well sorted, with $\sim 5 \%$ silt and clay matrix, slightly calcareous; 2 $3 \%$ glauconite, $2 \%$ silvery mica, colored heavies (tr), lignite (tr); fossils common, mostly pelecypods; texture massive; dark greenish gray $(5 G 4 / 1)$. Box 69. 


\section{0 - 730.0 ft: Run 91}

720.0 - $721.0 \mathrm{ft}$ : SAND, quartz, vf-f, well sorted, with 10-15\% silt and clay matrix, slightly calcareous; $5-10 \%$ silvery mica, chlorite (tr), glauconite (tr), colored heavy minerals (tr); abundant fossils, mostly pelecypods, some with nacreous luster; faint thin to wavy bedding; dark greenish gray $(5 G 4 / 1)$. Box 69.

721.0 - 721.6 ft: COQUINA, mostly pelecypods, with carbonate matrix; dark greenish gray $(5 G 4 / 1)$. Box 69.

721.6 - $722.4 \mathrm{ft}:$ SAND, quartz, vf-f, with $\sim 10 \%$ silt and clay matrix, slightly calcareous; 5\% silvery mica, glauconite, and chlorite; shells common, mostly pelecypods; sharp basal contact; dark greenish gray $(5 G 4 / 1)$. Box 69 and Box 70.

722.4 - $725.5 \mathrm{ft}$ : SILT, very clayey, contains $\sim 15 \% \mathrm{f}-\mathrm{c}$ quartz sand; $2-3 \%$ silvery mica, heavy minerals (tr); no obvious bedding, but root structures with degraded carbon and replacement pyrite present from 724.5 to $725.0 \mathrm{ft}$; gradational contact with bed below; greenish gray $(5 G Y 6 / 1)$. Box 70 .

725.5 - $729.3 \mathrm{ft}$ : SAND, quartz, f-m, with 20-25\% silt and clay matrix; $2-3 \%$ silvery mica, garnet (tr), opaque heavy minerals (tr), lignite fragments (tr); massive texture; greenish gray (5GY 6/1) mottled moderate yellowish brown (10YR 5/4). Box 70.

$729.3-730.0 \mathrm{ft}$ : No recovery.

\section{0 - 740.0 ft: Run 92}

730.0 - $732.0 \mathrm{ft}$ : SAND, quartz, vf-f, angular to subangular, with silt and clay matrix; massive texture except for crude thin bedding near base; gradational boundary with bed below; greenish gray (5GY 6/1) mottled moderate yellowish brown (10YR 5/4). Box 71 .

732.0 - $736.3 \mathrm{ft}:$ SAND, quartz, vf-f, angular to subangular, with silt and clay matrix; 5\% chlorite, silvery mica (tr), heavy minerals (tr); cross-bedded, herringbone pattern with layers $0.1 \mathrm{ft}$ thick; grayish green $(5 G \mathrm{Y} 6 / 1)$. Box 71.

736.3 - 738.6 ft: SILT, clayey, contains vf-f quartz sand, carbonaceous; laminated; dark gray (N 3). Box 71 and Box 72.

738.6 - $739.5 \mathrm{ft}$ : SAND, quartz, vf-f, angular to subangular, with silt and clay matrix; 5\% chlorite, silvery mica (tr), heavy minerals (tr); cross-bedded, herringbone pattern with layers $0.1 \mathrm{ft}$ thick; grayish green $(5 G Y 6 / 1)$. Box 72 .

739.5 - $740.0 \mathrm{ft}:$ No recovery.

\section{0 - 750.0 ft: Run 93}

740.0 - $742.9 \mathrm{ft}:$ SAND, quartz, vf-f, angular, with silt and clay matrix; $~ 5 \%$ silvery mica, 1-2\% lignite fragments up to $14 \mathrm{~mm}$ long, chlorite or glauconite (tr); interlayered with SILT, very clayey, micaceous; layers 2-10 mm thick, horizontal to slightly inclined, probable low-angle cross-beds in sand laminae; olive gray (5Y 4/1). Box 72.

742.9 - 746.2 ft: SAND, quartz, vf-f, angular, well sorted, contains $\sim 15 \%$ silt and clay matrix; 5-10\% silvery mica, chlorite/glauconite (tr), lignite clasts up to $4 \mathrm{~mm}$ in diameter (tr); thin bedding, some cross-bedded; sharp, inclined contact with bed below; dark greenish gray $(5 G 4 / 1)$. Box 72 . 
746.2 - 749.1 ft: SAND, quartz, vf-f, angular, well sorted; 3-5\% silvery mica, $1 \%$ glauconite, $\sim 1 \%$ opaque heavy minerals; massive texture with local burrows, $14 \mathrm{~mm}$ in diameter and filled with f-m quartz sand; dark greenish gray (5GY 4/1). Box 72 and Box 73.

$749.1-750.0 \mathrm{ft}$ : No recovery.

\section{0 - 759.0 ft: Run 94}

750.0 - $752.4 \mathrm{ft}$ : SAND, quartz, vf-f grading down to f-vc, subround, well sorted, contains 20-30\% silt and clay matrix; $5 \%$ chlorite, $2-3 \%$ silvery mica, $2 \%$ grading down to $10-15 \%$ glauconite, $1-2 \%$ phosphate near base; sand concentrated in burrows, some burrows partially cemented and slightly calcareous; dark greenish gray $(5 G Y 4 / 1)$. Box 73.

$752.4-757.0 \mathrm{ft}$ : No recovery. Sparse phosphate pebbles in core rind immediately above probably come from this interval. Geophysical log indicates fining down to $757.0 \mathrm{ft}$ and then abrupt change below that.

\section{Potomac Group, Patuxent Formation}

$757.0-759.0 \mathrm{ft}$ : No recovery. Geophysical log indicates this interval is quite different from interval immediately above.

\section{0 - 765.0 ft: Run 95}

759.0 - $762.0 \mathrm{ft}$ : SAND, quartz, vf-f, subround, well sorted, grading down to f-c, subround, poorly sorted, contains $10-20 \%$ silt and clay matrix; $10-12 \%$ chlorite, $2-3 \%$ silvery mica, $\sim 2 \%$ dark fine heavy minerals; massive texture with root structures present up to $5 \mathrm{~cm}$ in length; fining upward sequence; color comprised of mottles of yellowish brown (10YR 5/4), pale red (10R 6/2), and greenish gray (5GY 6/1). Box 73 and Box 74 .

762.0 - $765.0 \mathrm{ft}:$ No recovery.

\section{0 - 770.0 ft: Run 96}

765.0 - $770.0 \mathrm{ft}$ : SAND, quartz, f-m, subangular to subround, moderately sorted, with 5-7\% silt and clay matrix; $5-7 \%$ chlorite (m-vc size), $1-2 \%$ dark heavy minerals; cross-bedded with chlorite forming thin bands on drapes; sand coarsens slightly down section; greenish gray $(5 G Y 6 / 1)$ mottled moderate yellowish brown (10YR 5/4). Box 74 .

\section{0 - 780.0 ft: Run 97}

770.0 - $771.0 \mathrm{ft}$ : SAND, quartz, vf-m, subangular to subround, poorly sorted, with $<5 \%$ silt and clay matrix; 3-5\% chlorite, $~ 2 \%$ finely disseminated lignite; faintly cross-bedded in upper part; high porosity, no transmissivity, very dry; dark greenish gray (5GY 4/1). Box 74.

$771.0-780.0 \mathrm{ft}$ : No recovery. 


\section{0 - 784.0 ft: Run 98}

780.0 - $781.1 \mathrm{ft}:$ SAND, quartz, f-m, subround, well sorted, grading down to f-vc, subangular to subround, poorly sorted, with $<5 \%$ silt and clay matrix; 2-3\% dark heavy minerals, yellow quartz (tr), blue quartz (tr), garnet (tr), silvery mica (tr); massive texture; dark greenish gray $(5 G Y 4 / 1)$. Box 74 and Box 75.

$781.1-784.0 \mathrm{ft}:$ No recovery.

\section{0 - 790.0 ft: Run 99}

784.0 - $789.2 \mathrm{ft}$ : SAND, quartz, f-vc, angular to subangular, poorly sorted, with $<5 \%$ silt and clay matrtix; 5-7\% chlorite, $\sim 2 \%$ silvery mica, yellow quartz (tr), garnet (tr), 1-3\% lignite fragments up to $1 \mathrm{~cm}$ in length; modal grain size varies through section; massive texture; porosity variable, transmissivity low to moderate; dark greenish gray ( $5 G Y$ 4/1). Box 75.

789.2 - $790.0 \mathrm{ft}$ : No recovery.

\section{0 - 800.0 ft: Run 100}

790.0 - $791.7 \mathrm{ft}:$ SAND, quartz, f-vc, subangular, poorly sorted, with <5\% silt and clay matrix; rose quartz (tr), silvery mica (tr), garnet (tr), 1-2\% disseminated lignite and rare lignite pieces up to $1 \mathrm{~cm}$ in length; quartz granules and pebbles present near base; massive texture; olive gray $(5 Y$ 4/1). Box 75.

791.7 - $800.0 \mathrm{ft}$ : No recovery.

\section{0 - 810.0 ft: Run 101}

$800.0-810.0 \mathrm{ft}:$ No recovery.

\section{0 - 820.0 ft: Run 102}

810.0 - 812.4 ft: SILT, very clayey, contains thin blebs and stringers of vf-f, subangular, well sorted quartz sand; up to $10 \%$ lignite as chunks up t $2 \mathrm{~cm}$ in length and in thin layers up to $0.1 \mathrm{ft}$ thick; pyrite commonly replaces lignite and occurs in nodules up to $1.5 \mathrm{~cm}$ in diameter; clay most abundant in thin, discontinuous laminae; gradational contact with bed below; grayish black (N2). Box 76.

$812.4-814.7 \mathrm{ft}$ : SAND, quartz vf-vc, angular to subangular, poorly sorted, with $10-15 \%$ silt and clay matrix; lignite and pyrite nodules common; liquefaction-induced wavy bedding (could be original or drilling-induced); point-bar sequence; olive gray (5Y 4/1). Box 76.

814.7 - $817.0 \mathrm{ft}$ : SILT, contains 2-3\% vf-f subround, well sorted quartz and lignite sand; faint root and ped structures visible; thin layers of f-vc angular to subangular poorly sorted quartz sand present, with rutilated and yellow quartz pebbles up to $2 \mathrm{~cm}$ in diameter; tight and dry; olive gray $(5 Y$ 4/1). Box 76.

817.0 - $818.2 \mathrm{ft}$ : SILT, very clayey, contains 7-10\% vf-f rounded and well sorted quartz sand; disseminated lignite (tr); root casts and ped structures visible; tight and dry; top of a point-bar sequence; medium gray (N 5). Box 76 and Box 77. 
$818.2-820.0 \mathrm{ft}$ : No recovery.

\section{0 - 830.0 ft: Run 103}

820.0 - $820.2 \mathrm{ft}:$ SILT, very clayey, contains 7-10\% vf-f round, well sorted quartz sand; root casts and ped structures visible; disseminated lignite (tr); tight and dry; top of a point-bar sequence; medium gray ( $N 5)$. Box 77.

820.2 - $820.5 \mathrm{ft}$ : SAND, quartz, vf-f, round, well sorted, with silt and clay matrix; medium-gray ( $N$ 5). Box 77.

820.5 - $821.0 \mathrm{ft}:$ SILT, very clayey, contains 7-10\% vf-f round, well sorted quartz sand; root casts and ped structures visible; disseminated lignite (tr); tight and dry; top of a point-bar sequence; medium gray ( $N$ 5). Box 77.

821.0 - $822.3 \mathrm{ft}$ : SAND, quartz, f-vc, angular to subangular, poorly sorted, contains $<5 \%$ silt and clay matrix; $1-2 \%$ yellow and blue quartz; quartz granules and pebbles up to $1.5 \mathrm{~cm}$ in diameter common throughout; massive texture; light olive gray (5Y6/1). Box 77 .

$822.3-830.0 \mathrm{ft}$ : No recovery.

\section{0 - 840.0 ft: Run 104}

$8300-831.9 \mathrm{ft}$ : SAND, quartz, f-vc, angular to subangular, poorly sorted, with $<2 \%$ silt and clay matrix; yellow rutilated quartz and blue quartz grains common, garnets (tr); angular to subround quartz pebbles up to $4 \mathrm{~cm}$ in diameter abundant at base; high porosity and high transmissivity; yellowish gray (5Y7/2). Box 77.

831.9 - $832.5 \mathrm{ft}$ : SAND, quartz, f-c, subangular to subround, poorly sorted, with abundant clay and silt matrix, sticky; massive texture; contact gradational with bed below; greenish gray (5GY 6/1). Box 77.

832.5 - 833.2 ft: SAND, quartz, f-vc, angular to subangular, poorly sorted, with $<2 \%$ silt and clay matrix; yellow rutilated quartz and blue quartz grains common, garnets (tr); high porosity and high transmissivity; greenish gray (5GY 6/2). Box 77.

833.2 - $838.0 \mathrm{ft}$ : No recovery, geophysical log suggests sand as above.

838.0 - $840.0 \mathrm{ft}$ : No recovery, geophysical log suggests clayey silt as below.

\section{0 - 850.0 ft: Run 105}

$840.0-845.5 \mathrm{ft}:$ SILT, very clayey, contains 2-3\% vf quartz sand; root ghosts and very thin organic layers occur throughout; sharp contact with bed below; tight and dry, crumbles when cut; color ranges from dark-greenish gray $(5 G Y 4 / 1)$ to light olive gray $(5 Y 6 / 1)$. Box 77 and Box 78.

845.5 - 847.2 ft: SAND, quartz, f-vc, subangular to subround, poorly-sorted, with $<5 \%$ silt and clay matrix; $1-2 \%$ quartz granules, $1-2 \%$ blue quartz, rutilated quartz, and yellow quartz; massive texture; color comprised of mottles of dark greenish gray (6GY 4/1) and moderate yellowish brown (10YR 5/4). Box 78.

847.2 - $847.8 \mathrm{ft}$ : GRAVEL, quartz pebbles and granules, large silt rip-up clast present; sharp contact with bed below; dark greenish gray $(5 G Y$ 4/1). Box 78 .

847.8 - $848.5 \mathrm{ft}$ : SILT, very clayey, contains $2-3 \%$ vf quartz sand; paleosol, root ghosts and very thin organic layers occur throughout; tight and dry, crumbles when cut; color ranges from dark greenish gray $(5 G Y 4 / 1)$ to light olive gray $(5 Y 6 / 1)$. Box 78 .

848.5 - $850.0 \mathrm{ft}:$ No recovery. 


\section{0 - 860.0 ft: Run 106}

850.0 - $860.0 \mathrm{ft}$ : SILT, very clayey, contains <3\% vf quartz sand; ped structures and root casts present; slickensides present; sticky and crumbly; color comprised of mottles of greenish gray (5GY 6/1), moderate yellowish brown (10YR 5/4), and moderate reddish brown (10R 4/6). Box 78, Box 79, and Box 80.

\section{0 - 870.0 ft: Run 107}

860.0 - $866.2 \mathrm{ft}$ : SILT, very clayey, contains <3\% vf quartz sand; ped structures and root casts present; slickensides more abundant than above; sticky and crumbly; gradational contact with bed below; color comprised of mottles of greenish gray $(5 G Y 6 / 1)$, moderate yellowish brown (10YR 5/4), and moderate reddish brown (10R 4/6). Box 80 and Box 81.

866.2 - $870.0 \mathrm{ft}:$ SAND, quartz, vf-m, subround, moderately sorted, with $\sim 10 \%$ silt and clay matrix; $<2 \%$ disseminated pyrite, $\sim 1 \%$ dark heavy minerals, rutilated quartz (tr); massive texture; color comprised of mottles of greenish gray $(5 G 6 / 1)$ and moderate brown (5YR 3/4). Box 81.

\section{0 - 875.0 ft: Run 108}

870.0 - $871.0 \mathrm{ft}:$ SAND, quartz, vf-m, subround, moderately sorted, with $\sim 10 \%$ silt and clay matrix; $<2 \%$ disseminated pyrite, $\sim 1 \%$ dark heavy minerals, rutilated quartz (tr); massive texture; color comprised of mottles of greenish gray $(5 G 6 / 1)$ and moderate brown (5YR 3/4). Box 81.

871.0 - $873.4 \mathrm{ft}$ : SAND, quartz, vf-m, subangular to subround, poorly sorted, with $7-10 \%$ silt and clay matrix, grading down to vf-vc, subround, poorly sorted, with rare subround quartz granules, $\sim 5 \%$ silt and clay; $2-3 \%$ dark heavy minerals, $1 \%$ chlorite, color comprised of mottles of greenish gray (5GY 6/1) and moderate yellowish brown (10Y R5/4). Box 81 and Box 82.

873.4 - $875.0 \mathrm{ft}$ : No recovery.

\section{0 - 880.0 ft: Run 109}

875.0 - 879.2 ft: SAND, quartz, f-vc, angular to subangular, poorly sorted, with <3\% silt and clay matrix; quartz granules present at top, becoming more abundant downward; quartz pebbles up to $4 \mathrm{~cm}$ in length common in lower part, foliated gneiss pebbles up to 3 $\mathrm{cm}$ in length scattered throughout, gravelly in basal $0.5 \mathrm{ft}$; dusky yellow $(5 Y$ 6/4) from 875.0 to 880.0 , grading rapidly to greenish gray $(5 G Y 6 / 1)$. Box 82 .

$879.2-880.0 \mathrm{ft}$ : No recovery. 


\section{0 - 890.0 ft: Run 110}

880.0 - 881.2 ft: SAND, quartz, f-vc, angular to subangular, poorly sorted, with 3-5\% silt and clay matrix; granules common and pebbles up to $1.5 \mathrm{~cm}$ scattered throughout; light olive gray $(5 Y 6 / 1)$. Box 82.

881.2 - $881.7 \mathrm{ft}$ : GRAVEL, mostly quartz, subangular to subround, with f-vc angular to subangular poorly sorted quartz sand matrix; clasts up to $4 \mathrm{~cm}$ in length; sharp contact with bed below; light olive gray (5Y 6/1). Box 82.

881.7 - $882.4 \mathrm{ft}$ : SILT, very clayey, contains pockets of $\mathrm{f}-\mathrm{vc}$ angular to subangular poorly sorted quartz sand; feldspar fragments common and granules scattered throughout; fragmented and burrowed texture; crumbly and dry; dark gray (N 3). Box 82.

$882.4-883.5 \mathrm{ft}$ : SAND, quartz, f-m, subangular to subround, moderately sorted, with 5-7\% silt and clay matrix; cobbles at base; greenish gray (5GY 6/1). Box 82 and Box 83 .

$883.5-890.0 \mathrm{ft}$ : No recovery.

\section{0 - 900.0 ft: Run 111}

890.0 - $890.6 \mathrm{ft}:$ SAND, quartz, f-m, subangular to subround, moderately sorted, with 5-7\% silt and clay matrix; subangular to subround pebbles and cobbles moderately abundant, large cobble at top $0.2 \mathrm{ft}$ in length; greenish gray $(5 G Y$ 6/1). Box 83 .

890.6 - 892.4 ft: SAND, quartz, vf-f, round, well sorted, with 30-40\% silt and clay matrix; 1-2\% dark heavy minerals, silvery mica (tr); massive texture; greenish gray (5GY 6/1). Box 83.

892.4 - $892.8 \mathrm{ft}$ : GRAVEL, quartz, subangular to angular, contains vf-f well sorted round quartz sand matrix; clasts up to $2 \mathrm{~cm}$ in length; greenish gray (5GY 6/1). Box 83 .

892.8 - $893.5 \mathrm{ft}$ : SILT, very clayey, contains vf quartz sand in small blebs; tight and dry; sharp contact with bed below, marked thin iron-cemented zone; color comprised of mottles of dark yellowish brown $(10 Y R 4 / 2)$, dusky yellow green $(5 G Y 5 / 2)$, and moderate yellowish brown (10YR 5/4). Box 83.

893.5 - $893.9 \mathrm{ft}$ : SAND, quartz, vf-m, subround, moderately sorted, with 5-7\% silt and clay matrix; 1$2 \%$ dark heavy minerals, lignite (tr); greenish gray $(5 G Y 6 / 1)$. Box 83 .

893.9 - $894.3 \mathrm{ft}:$ SILT, very clayey; dark greenish gray $(5 G Y 4 / 1)$. Box 83 .

894.3 - $895.0 \mathrm{ft}$ : SAND, quartz, vf-m, subround, moderately sorted, with 5-7\% silt and clay matrix; 1 $2 \%$ dark heavy minerals, lignite (tr); greenish gray $(5 G Y 6 / 1)$. Box 83 .

895.0 - $900.0 \mathrm{ft}:$ No recovery.

\section{0 - 910.0 ft: Run 112}

900.0 - 900.6 ft: SAND, quartz, vf-m, subround, moderately sorted, with 5-7\% silt and clay matrix; 1$2 \%$ dark heavy minerals, lignite (tr); greenish gray (5GY 6/1). Box 83.

900.6 - $900.8 \mathrm{ft}$ : GRAVEL, quartz and chloritic quartz, subangular to subround, with vf-m subround quartz sand matrix; clasts up to $3 \mathrm{~cm}$ in diameter. Box 83.

900.8 - $902.0 \mathrm{ft}$ : SAND, quartz, f-vc, subangular to subround, poorly sorted, with $20-25 \%$ silt and clay matrix; quartz granules and small pebbles common; massive texture; sharp contact with bed below; greenish gray (5GY 6/1). Box 83 and Box 84 .

902.0 - $907.4 \mathrm{ft}$ : SILT, very clayey, contains 2-3\% f-m subround quartz sand; ped structures and limonite-filled root casts present; massive texture; dry and crumbly; color comprised of mottles of greenish gray (5GY 6/1) and dark reddish brown (10R 3/4). Box 84. 
$907.4-910.0 \mathrm{ft}:$ No recovery.

\section{0 - 919.5 ft: Run 113}

910.0 - $914.2 \mathrm{ft}$ : No recovery. Geophysical log indicates loss was in silt at top; on-site marker in photo assumed loss in sand at bottom.

914.2 - $918.2 \mathrm{ft}$ : SILT, very clayey, contains 2-3\% f-m subround quartz sand; ped structures and limonite-filled root casts present; massive texture; dry and crumbly; color comprised of mottles of greenish gray (5GY 6/1) and dark reddish brown (10R 3/4). Box 85.

918.2 - $919.5 \mathrm{ft}$ : SAND, quartz, f-vc, subangular, poorly sorted, with <5\% silt and clay matrix; quartz granules common, $1-2 \%$ dark heavy minerals, $\sim 1 \%$ feldspar, blue and rutilated quartz (tr), garnet (tr); massive texture; greenish gray (5GY 6/1). Box 85.

\section{5 - 921.5 ft: Run 114}

919.5 - $921.5 \mathrm{ft}:$ No recovery.

\section{5 - 930.0 ft: Run 115}

921.5 - $930.0 \mathrm{ft}$ : No recovery.

\section{0 - 935.0 ft: Run 116}

930.0 - $931.0 \mathrm{ft}$ : SILT, very clayey, contains <2\% vf quartz sand; ped structures and root casts present; contact gradational with bed below; very hard, crumbly, dry; reddish brown (10R 4/6) mottled greenish gray $(5 G Y 6 / 1)$. Box 85 .

931.0 - $932.6 \mathrm{ft}:$ SAND, quartz, f, subround, well sorted, with 5-7\% silt and clay matrix; dark heavy minerals (tr); massive texture; greenish gray (5GY 6/1). Box 85.

932.6 - $935.0 \mathrm{ft}$ : No recovery.

\section{0 - 944.0 ft: Run 117}

935.0 - $935.3 \mathrm{ft}: \mathrm{SAND}$, quartz, f, subround, well sorted, with 5-7\% silt and clay matrix; dark heavy minerals (tr); massive texture; greenish gray (5GY 6/1). Box 85 .

935.3 - $936.5 \mathrm{ft}$ : SILT, very clayey, contains <3\% vf quartz sand; thin chloritic quartz sand stringers scattered throughout; basal contact sharp, marked by a thin iron-cemented zone; hard, crumbly, dry; color comprised of mottles of dark greenish gray $((5 G Y 4 / 1)$, olive gray (5Y 4/1), and moderate reddish brown (10R 4/6). Box 85.

936.5 - $938.0 \mathrm{ft}$ : SAND, quartz, f-vc, subangular to subround, poorly sorted, with 5-7\% silt and clay matrix; mud balls and rip-up clasts abundant, up to $1.5 \mathrm{~cm}$ in length; color comprised of mottles of moderate yellowish brown (10YR 5/4), greenish gray $(5 G Y 6 / 1)$, moderate brown (5Y 4/4), and very light gray $(N 8)$. Box 86.

938.0 - $939.2 \mathrm{ft}:$ SAND, quartz, f-vc, subangular, with $<5 \%$ silt and clay matrix; $5-7 \%$ dark heavy minerals, yellow and rutilated quartz (tr); color comprised of mottles of moderate yellowish brown (10YR 5/4), greenish gray (5GY 6/1), moderate brown (5Y 4/4), and very light gray $(N 8)$. Box 86. 
939.2 - $941.2 \mathrm{ft}:$ SAND, quartz, m-vc, subangular, with $<5 \%$ silt and clay matrix, quartz granules common, rare pebbles up to $1 \mathrm{~cm}$; color comprised of mottles of moderate yellowish brown (10YR 5/4), greenish gray (5GY 6/1), moderate brown (5Y 4/4), and very light gray (N 8). Box 86.

941.2 - $944.0 \mathrm{ft}:$ No recovery.

\section{0 - 950.0 ft: Run 118}

944.0 - $944.8 \mathrm{ft}$ : SANDSTONE, quartz, m-vc, subangular, with <5\% silt and clay matrix, quartz granules and angular pebbles up to $2 \mathrm{~cm}$ in length, silica cemented; color comprised of mottles of moderate yellowish brown $(10 Y R 5 / 4)$, greenish gray $(5 G Y 6 / 1)$, moderate brown (5Y 4/4), and very light gray $(N 8)$. Box 86.

944.8 - $950.0 \mathrm{ft}$ : SILT, very clayey, contains <2\% vf quartz sand; root casts abundant; hard, crumbly; color comprised of mottles of dark yellowish orange (10R 6/6), medium light gray $(N$ 6), and reddish brown (10R 4/6). Box 86 and Box 87.

\section{0 - 955.5 ft: Run 119}

950.0 - 955.4 ft: SILT, very clayey, contains <2\% vf quartz sand; 1-2 mm-thick layers of dark, organic-rich clay present, root casts abundant; hard, crumbly; color comprised of mottles of dark yellowish orange (10YR 6/6), medium light gray $(N 6)$, and reddish brown (10R 4/6). Box 87 and Box 88.

$955.4-955.5 \mathrm{ft}:$ No recovery.

\section{5 - 960.0 ft: Run 120}

955.5 - $958.9 \mathrm{ft}$ : SILT, very clayey, contains <2\% vf quartz sand; 1-2 mm-thick layers of dark, organic-rich clay present in top of section; two thin ( $<02 \mathrm{~mm}$ thick) lignitic zones near base, magnetite octahedrals form a thin layer between these lignite layers; mostly massive texture; tight, dry; light greenish gray $(5 G Y 8 / 1)$ to greenish gray $(5 G Y 6 / 1)$ mottled pale reddish brown (10R 5/4). Box 88.

958.9 - $960.0 \mathrm{ft}:$ No recovery.

\section{0 - 968.0 ft: Run 121}

960.0 - $961.5 \mathrm{ft}:$ SILT, very clayey, contains <2\% vf quartz sand; massive texture; contact gradational with bed below; tight, dry; light greenish gray $(5 G Y 8 / 1)$ to greenish gray $(5 G Y 6 / 1)$ mottled pale reddish brown (10R 5/4). Box 88.

961.5 - $965.9 \mathrm{ft}$ : SAND, quartz, f-c, angular to subangular, poorly sorted, with 5-7\% silt and clay matrix; silvery mica (tr), dark heavy minerals (tr); chert nodule present at $964.1,3 \mathrm{~cm}$ in length; massive texture; contact gradational with bed below; color comprised of mottles of grayish green (10GY 5/2), greenish gray (5GY 6/1), light brown $(5 Y R 5 / 6)$, and light olive brown ((5Y 5/6). Box 88 and Box 89.

965.9 - $967.9 \mathrm{ft}$ : SILT, clayey, contains quartz sand, m-c, subangular, poorly sorted; small mudballs up to $1 \mathrm{~cm}$ in diameter abundant in basal foot; ped structures and limonite-filled fracture 
surfaces present; crumbly, dry; color comprised of mottles of light olive gray (5Y 5/2) and dark reddish brown (10R 3/4). Box 89.

967.9 - $968.0 \mathrm{ft}:$ No recovery.

\section{0 - 970.5 ft: Run 122}

968.0 - $969.6 \mathrm{ft}$ : No recovery. Geophysical log indicates loss was sand at top; on-site marker in photo assumed loss at bottom.

969.6 - $970.5 \mathrm{ft}$ : SILT, very clayey; ped structures and root casts present, slickensides common; color comprised of mottles of light olive gray (5Y 5/2) and dark reddish brown (10R 3/4).

Box 89.

\section{5 - 973.0 ft: Run 123}

970.5 - $973.0 \mathrm{ft}$ : SILT, very clayey; ped structures and root casts present, slickensides common; color comprised of mottles of light olive gray (5Y 5/2) and dark reddish brown (10R 3/4). Box 89 and Box 90.

\section{0 - 980.0 ft: Run 124}

973.0 - $978.3 \mathrm{ft}$ : SILT, very clayey; mostly massive but with a few horizons of packed sand-size clayballs; slickensided surfaces scattered throughout; dark areas probably degraded pyrite turned to iron-oxide nodules; light olive gray $(5 Y$ 6/1) mottled pale reddish brown $(10 R 5 / 4)$, dark reddish brown $(10 R 3 / 4)$, and moderate reddish brown $(10 R$ 4/6). Box 90.

978.3 - $980.0 \mathrm{ft}$ : No recovery.

\section{0 - 985.0 ft: Run 125}

980.0 - 983.6 ft: SILT, very clayey; mostly massive but with a few horizons of packed sand-size clayballs; slickensided surfaces scattered throughout; dark areas probably degraded pyrite turned to iron-oxide nodules; fractured, crumbly; light olive gray $(5 Y 6 / 1)$ mottled pale reddish brown (10R 5/4), dark reddish brown (10R 3/4), and moderate reddish brown (10R 4/6). Box 90 and Box 91.

983.6 - $985.0 \mathrm{ft}:$ No recovery.

\section{0 - 995.0 ft: Run 126}

985.0 - $987.8 \mathrm{ft}$ : SILT, very clayey; massive; slickensided surfaces scattered throughout; fractured, crumbly; light greenish gray (5GY 8/1) mottled moderate brown (5YR 4/4). Box 91.

987.8 - $989.0 \mathrm{ft}$ : SILT, very clayey; knobbly texture due to accumulation of clayballs or due to a spheroidal weathering pattern, average diameter of spheroids about $8 \mathrm{~mm}$; fractured, crumbly; quartz geode present at $988.7 \mathrm{ft}$, center filled with clear quartz crystals; dark reddish brown (10R 3/4). Box 91.

989.0 - $989.5 \mathrm{ft}$ : SILT, very clayey, plastic; faintly layered, layers 3-5 mm thick; light greenish gray (5GY 8/1) mottled moderate brown (5YR 4/4). Box 91. 
989.5 - 990.3 ft: SILT, very clayey; knobbly texture due to accumulation of clayballs or due to a spheroidal weathering pattern, average diameter of spheroids about $8 \mathrm{~mm}$; fractured, crumbly; dark reddish brown (10R 3/4). Box 91.

990.3 - $991.0 \mathrm{ft}$ : SILT, very clayey, plastic; faintly layered, layers 3-5 mm thick; light greenish gray (5GY 8/1) mottled moderate brown (5YR 4/4). Box 91 and Box 92.

991.0 - $992.0 \mathrm{ft}$ : SILT, very clayey; knobbly texture due to accumulation of clayballs or due to a spheroidal weathering pattern, average diameter of spheroids about $8 \mathrm{~mm}$; fractured, crumbly; dark reddish brown (10R 3/4). Box 92.

992.0 - $993.5 \mathrm{ft}$ : SILT, very clayey, plastic; faintly layered, layers 3-5 mm thick; pisolites or borrows at $991.0 \mathrm{ft}$; light greenish gray $(5 G Y 8 / 1)$ mottled moderate brown (5YR 4/4). Box 92.

993.5 - $994.7 \mathrm{ft}$ : SILT, very clayey; knobbly texture due to accumulation of clayballs or due to a spheroidal weathering pattern (primitive pisolites?), average diameter spheroids about $8 \mathrm{~mm}$; fractured, crumbly; clay-filled fractures run through some areas at a $45^{0}$ angle; dark reddish brown (10R 3/4). Box 92.

994.7 - $995.0 \mathrm{ft}:$ No recovery.

\section{0 - 1000.0 ft: Run 127}

995.0 - $995.5 \mathrm{ft}$ : SILT, very clayey; knobbly texture due to accumulation of clayballs or due to a spheroidal weathering pattern (primitive pisolites?), average diameter spheroids about $8 \mathrm{~mm}$; fractured, crumbly; clay-filled fractures run through some areas at a $45^{0}$ angle; dark reddish brown (10R 3/4). Box 92.

995.5 - $996.6 \mathrm{ft}$ : SILT, very clayey, plastic; faintly layered, layers 3-5 mm thick; light-greenish-gray (5GY 8/1) mottled moderate brown (5YR 4/4). Box 92.

996.6 - $996.7 \mathrm{ft}$ : SILT, very clayey; knobbly texture due to accumulation of clayballs or due to a spheroidal weathering pattern (primitive pisolites?), average diameter spheroids about $8 \mathrm{~mm}$; fractured, crumbly; dark reddish brown (10R 3/4). Box 92.

996.7 - $996.9 \mathrm{ft}$ : SILT, very clayey, plastic; faintly layered, layers 3-5 mm thick; light greenish gray (5GY 8/1) mottled moderate-brown (5YR 4/4). Box 92.

996.9 - $997.0 \mathrm{ft}$ : SILT, very clayey; knobbly texture due to accumulation of clayballs or due to a spheroidal weathering pattern (primitive pisolites?), average diameter spheroids about $8 \mathrm{~mm}$; fractured, crumbly; dark reddish brown (10R 3/4). Box 92.

997.0 - $997.2 \mathrm{ft}$ : SILT, very clayey, plastic; faintly layered, layers 3-5 mm thick; light greenish gray (5GY 8/1) mottled moderate brown (5YR 4/4). Box 92.

997.2 - $997.3 \mathrm{ft}$ : SILT, very clayey; knobbly texture due to accumulation of clayballs or due to a spheroidal weathering pattern (primitive pisolites?), average diameter spheroids about $8 \mathrm{~mm}$; fractured, crumbly; dark reddish brown (10R 3/4). Box 92.

997.3 - $998.4 \mathrm{ft}$ : SILT, very clayey, plastic; faintly layered, layers 3-5 mm thick; light greenish gray (5GY 8/1) mottled moderate brown (5YR 4/4). Box 92.

$998.4-1000.0 \mathrm{ft}$ : No recovery.

\section{0 - 1010.0 ft: Run 128}

1000.0 - $1000.2 \mathrm{ft}$ : Sparse recovery; isolated $0.2 \mathrm{ft}$ section of knobbly, very clayey silt caught in core barrel. Box 93.

1000.2 - $1010.0 \mathrm{ft}$ : No recovery. Driller reports this interval drilled like sand. 
1010.0 - 1015.0 ft: Run 129

1010.0 - $1012.0 \mathrm{ft}$ : No recovery. Driller reports this interval drilled like sand.

1012.0 - $1015.0 \mathrm{ft}$ : No recovery. Driller reports this interval drilled like sand, but geophysical SP log suggests this interval is gravel.

\section{0 - 1020.0 ft: Run 130}

1015.0 - 1015.3 ft: Sparse recovery. Driller reports this interval drilled like sand; slurry of subangular to subround coarse quartz sand and granules captured in core barrel; many quartz grains rutilated, others yellow, pink, clear, and a few blue (tr). Box 93.

1015.3 - $1020.0 \mathrm{ft}$ : No recovery.

1020.0 - 1021.0 ft: Run 131

1020.0 - $1021.0 \mathrm{ft}:$ SILT, very clayey; root mottled; greenish gray (5GY 6/1) blotched moderate reddish brown $(10 R 4 / 6)$; possibly recaptured from higher up? Box 93.

1021.0 - 1030.0 ft: Run 132

1021.0 - $1026.0 \mathrm{ft}$ : Sparse recovery. Residue in core barrel included subangular to subround quartz granules and pebbles up to $3 \mathrm{~cm}$ long; one quartz fragment (from chlorite schist) 6 mm long. Box 93.

\section{Newark Supergroup, Chatham Group undifferentiated}

1026.0 - $1030.0 \mathrm{ft}$ : No recovery. Drilling mud changed from tan to bright-reddish-orange in this interval; big jump in both gamma and resistivity logs at $1026.0 \mathrm{ft}$ indicates major change in lithology.

\section{0 - 1032.0 ft: Run 133}

1030.0 - $1032.0 \mathrm{ft}$ : No recovery. Inner barrel failed to lock in core barrel so $2.0 \mathrm{ft}$ of core stuck in end of core barrel. Core pushed out with small drill rod, sample caught below probably from this interval.

\section{0 - 1035.0 ft: Run 134}

$1032.0-1032.7 \mathrm{ft}$ : Sparse recovery. $0.6 \mathrm{ft}$ of core or residue collected behind subround milky quartz cobble ( 55 x $40 \times 35 \mathrm{~mm}$ ) badly scoured by carbide wafers; probable cause of previous core loss; $0.6 \mathrm{ft}$ interval of dark reddish brown $(10 R 3 / 4)$ very clayey silt containing small fragments of dark reddish brown $(10 R 3 / 4)$ ferruginous sandstone. Box 93.

$1032.7-1035.0 \mathrm{ft}$ : No recovery. 
1035.0 - 1040.0 ft: Run 135

1035.0 - $1040.0 \mathrm{ft}$ : Sparse recovery. Rounded but broken yellowish gray (5Y 7/2) sandstone or quartzite cobble ( 45 x 35 × $30 \mathrm{~mm}$ ) caught in end of core barrel. Residue from blockage is sandy mud ranging from moderate red $(5 R 4 / 6)$ to dark reddish brown (10R 3/4). Box 93.

\section{0 - 1045.0 ft: Run 136}

1040.0 - $1045.0 \mathrm{ft}$ : Sparse recovery. Pulled up one large block of foliated quartzite $(80$ x 60 x $50 \mathrm{~mm})$ followed by $1.7 \mathrm{ft}$ of finer residue from base of section, dark reddish brown (10R 3/4) with pebbles stained reddish brown (10R 4/3). Box 93.

\section{0 - 1046.0 ft: Run 137}

1045.0 - $1046.0 \mathrm{ft}$ : SILT, very clayey; abundant root mottling; faintly banded or bedded; pale reddish brown (10R 6/4) mottled moderate reddish brown (10R 4/6). Box 93 and Box 94.

\section{0 - 1052.0 ft: Run 138}

1046.0 - 1046.7 ft: SILT, very clayey; abundant root mottling; faintly banded or bedded; dark reddish brown (10R 3/4). Box 94.

1046.7 - $1050.0 \mathrm{ft}:$ SILT, very clayey; massive; semi-indurated; grayish red (5R 4/2) mottled dark reddish brown (10R 3/4). Box 94.

1050.0 - 1050.2 ft: CONGLOMERATE, angular pebbles up to $1 \mathrm{~cm}$ in length in silt-clay matrix; background color yellowish gray ( $5 Y 7 / 2$ ), but some individual clasts black, green, and slate gray. Box 94.

1050.2 - $1050.4 \mathrm{ft}:$ SILT, very clayey; massive; semi-indurated; grayish red (5R 4/2) mottled dark reddish brown (10R 3/4). Box 94.

1050.4 - $1050.7 \mathrm{ft}$ : CONGLOMERATE, angular pebbles up to $1 \mathrm{~cm}$ in length in silt-clay matrix; background color yellowish gray ( $5 Y 7 / 2)$, but some individual clasts black, green, and slate gray. Box 94.

1050.7 - $1051.5 \mathrm{ft}$ : SILT, very clayey; massive; moderate reddish brown (10R 4/6) mottled grayish red (5R 4/2). Box 94.

1051.5 - $1052.0 \mathrm{ft}$ : SILT, clayey; includes a lense of pebble conglomerate like above and scattered angular pebbles or rock chips up to $4 \mathrm{~cm}$ long; moderate- reddish brown $(10 R \mathrm{4} / 6)$. Box 94.

\section{0 - 1060.0 ft: Run 139}

1052.0 - $1054.2 \mathrm{ft}$ : CONGLOMERATE, angular to subround pebbles and cobbles up to $6 \mathrm{~cm}$ long, very poorly sorted, clast supported; about $75 \%$ ferruginous Triassic sandstone clasts and 25\% Carolina Slate Belt schist and vein quartz clasts; some metamorphic clasts saprolitized and a few granitic clasts partly saprolitized; with vf-vc silty and clayey quartz sand matrix; this interval much fresher and denser than intervals above; matrix dark reddish brown (10R 3/4) to dusky red (5R 3/4); 
clasts varicolored including dark reddish brown (10R 3/4) Triassic clasts and moderate olive brown (5Y 4/4) Carolina Slate Belt clasts. Box 94 and Box 95.

1054.2 - $1055.9 \mathrm{ft}$ : SANDSTONE, quartz, $\mathrm{f}-\mathrm{m}$ with scattered $\mathrm{c}$ grading down to $\mathrm{m}-\mathrm{c}$ and quartz granular, round, with $10-15 \%$ silt and clay matrix; massive texture; dark reddish brown (10R 3/4). Box 95.

1055.9 - $1056.7 \mathrm{ft}$ : CONGLOMERATE, angular to subround pebbles and cobbles up to $6 \mathrm{~cm}$ long, very poorly sorted, clast supported; about $75 \%$ ferruginous Triassic sandstone clasts and 25\% Carolina Slate Belt schist, vein quartz, and diabase (or greenstone or hornfels) clasts; some metamorphic clasts saprolitized and a few granitic clasts partly saprolitized; with vf-vc silty and clayey quartz sand matrix; this interval much fresher and denser than intervals above; matrix dark reddish brown (10R 3/4) to dusky red (5R 3/4), clasts varicolored including dark reddish brown (10R 3/4) (Triassic), moderate olive brown (5Y 4/4) (Carolina Slate Belt), and dark gray $(N$ 3) (diabase). Box 95.

1056.7 - $1060.0 \mathrm{ft}$ : No recovery.

\section{0 - 1063.0 ft: Run 140}

1060.0 - $1061.4 \mathrm{ft}$ : CONGLOMERATE, mostly angular to subround pebbles up to $3 \mathrm{~cm}$ long, very poorly sorted, clast supported; about $75 \%$ ferruginous Triassic sandstone clasts and $25 \%$ Carolina Slate Belt schist, vein quartz, and diabase (or greenstone or hornfels) clasts; some metamorphic clasts saprolitized and a few granitic clasts partly saprolitized; vf-vc silty and clayey quartz sand matrix; this interval much fresher and denser than intervals above; matrix dark reddish brown $(10 R 3 / 4)$ to dusky red $(5 R 3 / 4)$, clasts varicolored including dark reddish brown (10R 3/4) Triassic clasts, moderate olive brown (5Y 4/4) Carolina Slate Belt clasts, and dark gray (N 3) diabase clasts. Box 95.

$1061.4-1063.0 \mathrm{ft}$ : No recovery.

\section{0 - 1070.0 ft: Run 141}

1063.0 - $1064.5 \mathrm{ft}$ : SILTSTONE, clayey, contains sparse vf quartz sand; semi-indurated; small chips of Carolina Slate Belt rocks scattered throughout; abundant root ghosts with pyrite cores oxidized to iron-oxide; massive texture; dark reddish brown $(10 R 3 / 4)$ with pale greenish yellow (10Y 8/2) root ghosts and blotches of grayish red purple (5RP 4/2). Box 95.

1064.5 - $1065.2 \mathrm{ft}$ : SANDSTONE, quartz, f-vc, subround, granular, semi-indurated; small chips of Carolina Slate Belt rocks scattered throughout; massive texture; moderate reddish brown $(10 R$ 4/6) mottled pale greenish yellow (10Y 8/2). Box 95.

1065.2 - $1067.1 \mathrm{ft}$ : SILTSTONE, clayey, contains sparse vf quartz sand; semi-indurated; small chips of Carolina Slate Belt rocks scattered throughout; abundant root ghosts with pyrite cores oxidized to iron-oxide; massive texture; dark reddish brown $(10 R 3 / 4)$ with pale greenish yellow (10Y 8/2) root ghosts and blotches of grayish red purple (5RP 4/2). Box 95 and Box 96.

1067.1 - $1067.7 \mathrm{ft}$ : No recovery. 
1067.7 - 1070.0 ft: SILTSTONE, clayey, contains $\sim 10 \%$ vf-vc quartz sand and $~ 2 \%$ quartz granules; massive texture; dark reddish brown (10R 3/4). (Recovered on Run 142, break placed at point of core undercut.) Box 96.

\section{0 - 1078.0 ft: Run 142}

1070.0 - $1070.9 \mathrm{ft}: \quad$ SILTSTONE, clayey, contains $~ 10 \%$ vf-vc quartz sand and $~ 2 \%$ quartz granules; massive texture; dark reddish brown (10R 3/4). Box 96.

1070.9 - $1072.5 \mathrm{ft}$ : CONGLOMERATE, angular to well rounded pebbles up to $4.5 \mathrm{~cm}$ long, clast supported; $\sim 50 \%$ ferruginous sandstone clasts and 50\% chloritic clasts, rare dark (probably diabase) clasts; vf-vc silty and clayey quartz sand matrix; ferruginous clasts grayish red $(5 R 4 / 2)$, chloritic clasts grayish olive (10Y $4 / 2)$, and diabase clasts olive black (5Y2/1). Box 96.

1072.5 - 1073.0 ft: SILTSTONE, clayey, contains $\sim 10 \%$ vf-vc quartz sand and $\sim 2 \%$ quartz granules; massive texture; dark reddish brown (10R 3/4). Box 96.

1073.0 - $1074.9 \mathrm{ft}$ : CONGLOMERATE, angular to well rounded pebbles up to $4.5 \mathrm{~cm}$ long, clast supported; $~ 50 \%$ ferruginous sandstone clasts and 50\% chloritic clasts, rare dark (probably diabase) clasts; vf-vc silty and clayey quartz sand matrix; ferruginous clasts grayish red $(5 R 4 / 2)$, chloritic clasts grayish olive (10Y 4/2), and diabase clasts olive black (5Y 2/1). Box 96 and Box 97.

1074.9 - $1075.3 \mathrm{ft}$ : SILTSTONE, clayey, contains $\sim 10 \%$ vf-vc quartz sand and $\sim 2 \%$ quartz granules; massive texture; prominent slickensided fracture cuts at $45^{\circ}$ angle through this section; dark reddish brown (10R 3/4). Box 97.

1075.3 - $1078.0 \mathrm{ft}$ : CONGLOMERATE, angular to round pebbles up to $4.5 \mathrm{~cm}$ long, matrix supported; 75\% chloritic clasts and $\sim 25 \%$ ferruginous sandstone clasts; f-vc silty and clayey quartz sand matrix; chloritic clasts greenish gray (5GY 6/1), ferruginous clasts grayish red (5R 4/2), sand matrix dark reddish brown (10R 3/4). Box 97.

\section{0 - 1085.0 ft: Run 143}

1078.0 - $1079.8 \mathrm{ft}$ : SILTSTONE, very clayey; ferruginous sandstone clasts in basal $0.1 \mathrm{ft}$; massive texture; jointed and fractured, surfaces thinly coated with pale green mineral; dark reddish brown (10R 3/4) to dusky red (5R 3/4). Box 97.

$1079.8-1080.0 \mathrm{ft}$ : No recovery.

1080.0 - $1080.3 \mathrm{ft}$ : SILTSTONE, clayey, contains vf quartz sandy; massive texture; grayish-red (5R 4/2). Box 97.

1080.3 - $1082.9 \mathrm{ft}$ : CONGLOMERATE, subround to round pebbles up to $4 \mathrm{~cm}$ long, clast supported; $\sim 50 \%$ ferruginous sandstone clasts and $\sim 50 \%$ chloritic clasts; f-vc silty and clayey quartz sand matrix; sandstone clasts dusky red ( $5 R 3 / 4)$, chloritic clasts yellowish gray (5Y 7/2), sand matrix dark reddish brown (10R 3/4). Box 97 and Box 98.

1082.9 - $1085.0 \mathrm{ft}$ : SILTSTONE, clayey, vf quartz sandy; contains $\sim 5 \% \mathrm{c}$-vc quartz and rock fragment sand and granules in thin zones; grayish red (5R 4/2). Box 98.

\section{0 - 1090.0 ft: Run 144}

1085.0 - $1088.5 \mathrm{ft}$ : SILTSTONE, clayey, contains vf quartz sand; $\sim 5 \% \mathrm{c}-\mathrm{vc}$ quartz and rock fragment sand and granules in thin zones; root mottling from 1085.5 to $1088.0 \mathrm{ft}$; thin $(\sim 1$ 
$\mathrm{mm}$ wide) mineralized layers cross core at 1085.0 and $1086.3 \mathrm{ft}$; possibly zeolites; filled void at $185.7 \mathrm{ft}$; grayish red $(5 R 4 / 2)$. Box 98.

1088.5 - $1090.0 \mathrm{ft}$ : CONGLOMERATE, subround to round pebbles and cobbles up to $9 \mathrm{~cm}$ in length, clast supported; diverse clast types including foliated slate/schist flat pebbles, ferruginous sandstone clasts, and granitic clasts; f-vc silty and clayey quartz sand matrix; clast colors as above plus many more, sand matrix dark reddish brown (10R 3/4). Box 98 and Box 99.

\section{0 - 1094.5 ft: Run 145}

1090.0 - $1091.9 \mathrm{ft}$ : CONGLOMERATE, subround to round pebbles and cobbles up to $8.4 \mathrm{~cm}$ in length, clast supported; diverse clast types including foliated slate/schist flat pebbles, ferruginous sandstone clasts, and granitic clasts; f-vc silty and clayey quartz sand matrix; slickensides locally present; clast colors as above plus many more, sand matrix dark reddish brown (10R 3/4). Box 99.

1091.9 - $1092.7 \mathrm{ft}$ : SANDSTONE, f-vc, subangular to subround, poorly sorted, with silt and clay matrix; some vc grains are fragments of Carolina Slate Belt lithologies; knobbly texture; very dusky red purple (5RP 2/2). Box 99.

1092.7 - 1093.2 ft: SANDSTONE, f-vc, subangular to subround, poorly sorted, with silt and clay matrix; some vc grains are fragments of Carolina Slate Belt lithologies; massive texture; dark reddish brown (10R 3/4). Box 99.

1093.2 - $1094.5 \mathrm{ft}$ : No recovery.

\section{Bottom of Hole: Total Depth 1094.5 ft.}


APPENDIX 2. Hope Plantation run log, showing run numbers, time of extrusion from the inner core barrel, depth, recovery, and any other significant information pertaining to core loss.

\section{Hope Plantation - Run Log}

\begin{tabular}{|c|c|c|c|c|c|c|c|}
\hline Date & Run \# & Time & $\begin{array}{c}\text { Top } \\
\text { Depth (ft) }\end{array}$ & $\begin{array}{l}\text { Bottom } \\
\text { Depth (ft) }\end{array}$ & $\begin{array}{l}\text { Core } \\
\text { Drilled }\end{array}$ & $\begin{array}{c}\text { Core } \\
\text { Recovered }\end{array}$ & Notes \\
\hline 3/12/04 & 1 & 8:55am & 0.0 & 3.0 & 3.0 & 1.9 & 1.1 loss at bottom \\
\hline $3 / 12 / 04$ & 2 & 9:10am & 3.0 & 7.0 & 4.0 & 1.5 & 2.5 loss at bottom \\
\hline $3 / 12 / 04$ & 3 & 9:30am & 7.0 & 12.0 & 5.0 & 2.0 & 3.0 loss at bottom \\
\hline 3/12/04 & 4 & $10: 15 \mathrm{am}$ & 12.0 & 15.0 & 3.0 & 1.6 & 1.4 loss at bottom \\
\hline 3/12/04 & 5 & 10:40am & 15.0 & 20.0 & 5.0 & 2.7 & 2.3 loss at bottom-core compaction \\
\hline $3 / 12 / 04$ & 6 & 11:30am & 20.0 & 24.0 & 4.0 & 0.0 & 4.0 loss-very loose coarse sand \\
\hline $3 / 12 / 04$ & 7 & 11:50am & 24.0 & 29.0 & 5.0 & 0.0 & 5.0 loss - gravel in shoe \\
\hline $3 / 12 / 04$ & 8 & $12: 15 \mathrm{pm}$ & 29.0 & 30.5 & 1.5 & 1.4 & 0.1 loss at bottom \\
\hline $3 / 12 / 04$ & 9 & $12: 50 \mathrm{pm}$ & 30.5 & 34.0 & 3.5 & 3.2 & 0.2 loss at bottom \\
\hline $3 / 12 / 04$ & 10 & $2: 30 \mathrm{pm}$ & 34.0 & 40.0 & 6.0 & 5.2 & 0.8 loss at bottom \\
\hline $3 / 13 / 04$ & 11 & 10:30am & 40.0 & 50.0 & 10.0 & 9.8 & 0.2 loss at bottom \\
\hline $3 / 13 / 04$ & 12 & $11: 15 \mathrm{am}$ & 50.0 & 60.0 & 10.0 & 9.5 & 0.5 loss at bottom \\
\hline $3 / 13 / 04$ & 13 & $12: 15 \mathrm{pm}$ & 60.0 & 70.0 & 10.0 & 9.7 & 0.3 loss at bottom \\
\hline $3 / 13 / 04$ & 14 & $12: 45 \mathrm{pm}$ & 70.0 & 80.0 & 10.0 & 10.2 & 0.2 extra - core expansion \\
\hline $3 / 13 / 04$ & 15 & $1: 30 \mathrm{pm}$ & 80.0 & 90.0 & 10.0 & 4.0 & 6.0 loss at bottom \\
\hline $3 / 13 / 04$ & 16 & $2: 15 \mathrm{pm}$ & 90.0 & 95.0 & 5.0 & 4.9 & 0.1 loss at bottom \\
\hline $3 / 13 / 04$ & 17 & $2: 45 \mathrm{pm}$ & 95.0 & 100.0 & 5.0 & 3.3 & 1.7 loss at bottom \\
\hline $3 / 13 / 04$ & 18 & $4: 15 \mathrm{pm}$ & 100.0 & 110.0 & 10.0 & 10.7 & 0.7 - core expansion \\
\hline $3 / 13 / 04$ & 19 & $5: 05 \mathrm{pm}$ & 110.0 & 120.0 & 10.0 & 8.3 & 1.7 loss at bottom \\
\hline $3 / 14 / 04$ & 20 & $9: 15 \mathrm{am}$ & 120.0 & 121.5 & 1.5 & 0.0 & 1.5 loss - pyrite nodule \\
\hline $3 / 14 / 04$ & 21 & $9: 55 \mathrm{am}$ & 121.5 & 130.0 & 8.5 & 8.9 & 0.4 extra from previous run \\
\hline $3 / 14 / 04$ & 22 & $10.30 \mathrm{am}$ & 130.0 & 140.0 & 10.0 & 10.0 & \\
\hline $3 / 14 / 04$ & 23 & $10.55 \mathrm{am}$ & 140.0 & 150.0 & 10.0 & 10.6 & 0.6 extra - core expansion \\
\hline $3 / 14 / 04$ & 24 & $11: 45 \mathrm{am}$ & 150.0 & 160.0 & 10.0 & 6.4 & 3.6 loss at top \\
\hline $3 / 14 / 04$ & 25 & $12: 15 \mathrm{pm}$ & 160.0 & 162.0 & 2.0 & 1.8 & 0.2 loss at bottom \\
\hline $3 / 14 / 04$ & 26 & $2: 05 \mathrm{pm}$ & 162.0 & 170.0 & 8.0 & 5.0 & 3.0 loss at bottom \\
\hline $3 / 14 / 04$ & 27 & $2: 45 \mathrm{pm}$ & 170.0 & 180.0 & 10.0 & 3.3 & 6.7 loss at bottom \\
\hline $3 / 14 / 04$ & 28 & $3: 50 \mathrm{pm}$ & 180.0 & 190.0 & 10.0 & 5.8 & 4.2 loss at top \\
\hline $3 / 14 / 04$ & 29 & $5: 15 \mathrm{pm}$ & 190.0 & 200.0 & 10.0 & 4.5 & 5.5 loss at bottom \\
\hline 3/15/04 & 30 & $9: 26 \mathrm{am}$ & 200.0 & 205.0 & 5.0 & 4.2 & 0.8 loss at bottom \\
\hline 3/15/04 & 31 & 10:15am & 205.0 & 210.0 & 5.0 & 4.9 & 0.1 loss at bottom \\
\hline $3 / 15 / 04$ & 32 & $12: 05 \mathrm{pm}$ & 210.0 & 220.0 & 10.0 & 8.9 & 1.1 loss at bottom \\
\hline $3 / 15 / 04$ & 33 & $1: 05 \mathrm{pm}$ & 220.0 & 230.0 & 10.0 & 3.6 & 6.4 loss at bottom \\
\hline 3/15/04 & 34 & $2: 40 \mathrm{pm}$ & 230.0 & 240.0 & 10.0 & 3.9 & 6.1 loss at bottom \\
\hline 3/15/04 & 35 & $3: 25 \mathrm{pm}$ & 240.0 & 250.0 & 10.0 & 8.2 & 1.8 loss at bottom \\
\hline
\end{tabular}




\begin{tabular}{|c|c|c|c|c|c|c|c|}
\hline $3 / 15 / 04$ & 36 & $4: 25 \mathrm{pm}$ & 250.0 & 260.0 & 10.0 & 6.3 & 3.7 loss at bottom \\
\hline $3 / 15 / 04$ & 37 & $4: 55 \mathrm{pm}$ & 260.0 & 270.0 & 10.0 & 2.2 & 7.8 loss at bottom \\
\hline 3/16/04 & 38 & $9: 15 \mathrm{am}$ & 270.0 & 276.0 & 6.0 & 2.6 & 3.4 loss at bottom \\
\hline $3 / 16 / 04$ & 39 & $9: 45 \mathrm{am}$ & 276.0 & 283.0 & 7.0 & 6.4 & 0.6 loss at bottom \\
\hline $3 / 16 / 04$ & 40 & 10:45am & 283.0 & 290.0 & 7.0 & 2.9 & 4.1 loss at bottom \\
\hline $3 / 16 / 04$ & 41 & $12: 00 \mathrm{pm}$ & 290.0 & 300.0 & 10.0 & 5.9 & 4.1 loss at bottom \\
\hline 3/17/04 & 42 & $9: 15 \mathrm{am}$ & 300.0 & 310.0 & 10.0 & 9.8 & 0.2 loss at bottom \\
\hline $3 / 17 / 04$ & 43 & 10:05am & 310.0 & 315.0 & 5.0 & 5.1 & 0.1 extra - core expansion \\
\hline 3/17/04 & 44 & 10:50am & 315.0 & 320.0 & 5.0 & 4.8 & 0.2 loss at bottom \\
\hline $3 / 17 / 04$ & 45 & 11:40am & 320.0 & 330.0 & 10.0 & 10.3 & 0.3 extra - core expansion \\
\hline $3 / 17 / 04$ & 46 & $1: 00 \mathrm{pm}$ & 330.0 & 340.0 & 10.0 & 7.8 & 2.2 loss at bottom-core slid out, retrieved it \\
\hline $3 / 17 / 04$ & 47 & $2: 40 \mathrm{pm}$ & 340.0 & 350.0 & 10.0 & 9.5 & 0.5 loss at bottom \\
\hline $3 / 17 / 04$ & 48 & 4:00pm & 350.0 & 360.0 & 10.0 & 3.3 & 6.7 loss at bottom \\
\hline $3 / 17 / 04$ & 49 & $5: 10 \mathrm{pm}$ & 360.0 & 363.0 & 3.0 & 2.1 & 0.9 loss at bottom \\
\hline 3/18/04 & 50 & 10:40am & 363.0 & 370.0 & 7.0 & 8.0 & picked up yesterdays loss \\
\hline $3 / 18 / 04$ & 51 & $12: 10 \mathrm{pm}$ & 370.0 & 380.0 & 10.0 & 7.7 & 2.3 loss - recovered in run 52 \\
\hline $3 / 18 / 04$ & 52 & $1: 45 \mathrm{pm}$ & 380.0 & 390.0 & 10.0 & 4.4 & 5.6 loss at bottom \\
\hline $3 / 18 / 04$ & 53 & $2: 55 \mathrm{pm}$ & 390.0 & 400.0 & 10.0 & 10.0 & \\
\hline $3 / 18 / 04$ & 54 & $3: 45 \mathrm{pm}$ & 400.0 & 410.0 & 10.0 & 7.7 & 2.3 loss at bottom \\
\hline $3 / 18 / 04$ & 55 & 5:00pm & 410.0 & 420.0 & 10.0 & 2.2 & 7.8 loss at bottom \\
\hline 3/19/04 & 56 & 9:00am & 420.0 & 430.0 & 10.0 & 6.0 & 4.0 loss at bottom \\
\hline $3 / 19 / 04$ & 57 & $9: 45 \mathrm{am}$ & 430.0 & 440.0 & 10.0 & 5.2 & 4.8 loss at bottom \\
\hline $3 / 19 / 04$ & 58 & 10:55am & 440.0 & 450.0 & 10.0 & 8.7 & 1.3 loss at bottom \\
\hline $3 / 19 / 04$ & 59 & $12: 30 \mathrm{pm}$ & 450.0 & 460.0 & 10.0 & 8.6 & 1.4 loss at bottom \\
\hline $3 / 19 / 04$ & 60 & $2: 20 \mathrm{pm}$ & 460.0 & 470.0 & 10.0 & 9.1 & 0.9 loss at bottom \\
\hline $3 / 19 / 04$ & 61 & $3: 20 \mathrm{pm}$ & 470.0 & 480.0 & 10.0 & 2.8 & 7.2 loss at bottom \\
\hline $3 / 19 / 04$ & 62 & $5: 15 \mathrm{pm}$ & 480.0 & 490.0 & 10.0 & 4.6 & 5.4 loss at bottom \\
\hline $3 / 20 / 04$ & 63 & $9: 15 \mathrm{am}$ & 490.0 & 500.0 & 10.0 & 9.3 & 0.7 loss at bottom \\
\hline $3 / 20 / 04$ & 64 & $11: 15 \mathrm{am}$ & 500.0 & 510.0 & 10.0 & 10.4 & $0.4 \mathrm{ft}$. extra - crumbled core, excess space \\
\hline $3 / 20 / 04$ & 65 & $12: 45 \mathrm{pm}$ & 510.0 & 520.0 & 10.0 & 8.5 & 1.5 loss at bottom \\
\hline $3 / 20 / 04$ & 66 & $2: 30 \mathrm{pm}$ & 520.0 & 530.0 & 10.0 & 6.2 & 3.8 loss at bottom \\
\hline $3 / 20 / 04$ & 67 & $5: 30 \mathrm{pm}$ & 530.0 & 540.0 & 10.0 & 4.2 & 5.8 loss at bottom \\
\hline $3 / 21 / 04$ & 68 & 8:40am & 540.0 & 550.0 & 10.0 & 3.9 & 6.1 loss at bottom \\
\hline $3 / 21 / 04$ & 69 & 9:40am & 550.0 & 560.0 & 10.0 & 3.1 & 6.9 loss at bottom \\
\hline $3 / 21 / 04$ & 70 & $10.25 \mathrm{am}$ & 560.0 & 570.0 & 10.0 & 2.4 & 7.6 loss at bottom \\
\hline $3 / 21 / 04$ & 71 & $12: 00 \mathrm{pm}$ & 570.0 & 580.0 & 10.0 & 9.8 & 0.2 loss at bottom \\
\hline $3 / 21 / 04$ & 72 & $1: 35 \mathrm{pm}$ & 580.0 & 583.0 & 3.0 & 2.5 & 0.5 loss at bottom \\
\hline $3 / 21 / 04$ & 73 & $2: 55 \mathrm{pm}$ & 583.0 & 590.0 & 7.0 & 7.2 & 0.2 extra - core expansion \\
\hline $3 / 21 / 04$ & 74 & $4: 30 \mathrm{pm}$ & 590.0 & 600.0 & 10.0 & 9.2 & 0.8 loss at bottom \\
\hline $3 / 22 / 04$ & 75 & 9:30am & 600.0 & 610.0 & 10.0 & 9.0 & 1.0 loss at bottom \\
\hline $3 / 22 / 04$ & 76 & 10:50am & 610.0 & 620.0 & 10.0 & 9.5 & 0.5 loss at bottom \\
\hline $3 / 22 / 04$ & 77 & $1: 10 \mathrm{pm}$ & 620.0 & 630.0 & 10.0 & 9.0 & 1.0 loss at bottom \\
\hline $3 / 22 / 04$ & 78 & $3: 45 \mathrm{pm}$ & 630.0 & 640.0 & 10.0 & 1.4 & 8.6 loss at bottom \\
\hline $3 / 22 / 04$ & 79 & 5:00pm & 640.0 & 646.5 & 6.5 & 6.6 & 0.1 extra - core expansion \\
\hline $3 / 23 / 04$ & 80 & $12: 20 \mathrm{pm}$ & 646.5 & 650.0 & 3.5 & 3.3 & 0.2 loss at bottom \\
\hline
\end{tabular}




\begin{tabular}{|c|c|c|c|c|c|c|c|}
\hline $3 / 23 / 04$ & 81 & $2: 00 \mathrm{pm}$ & 650.0 & 654.3 & 4.3 & 4.3 & \\
\hline $3 / 23 / 04$ & 82 & $4: 15 \mathrm{pm}$ & 654.3 & 660.0 & 5.7 & 6.3 & 0.6 extra? \\
\hline $3 / 24 / 04$ & 83 & & 660.0 & 670.0 & 10.0 & 10.2 & 0.2 extra \\
\hline 4/4/04 & 84 & & 670.0 & 678.5 & 8.5 & 8.3 & 0.2 loss at bottom \\
\hline $4 / 404$ & 85 & & 678.5 & 679.7 & 1.2 & 0.9 & 0.3 loss at bottom \\
\hline $4 / 4 / 04$ & 86 & & 679.7 & 690.0 & 10.3 & 10.5 & 0.2 extra \\
\hline $4 / 4 / 04$ & 87 & & 690.0 & 700.0 & 10.0 & 10.1 & 0.1 extra \\
\hline $4 / 4 / 04$ & 88 & & 700.0 & 710.0 & 10.0 & 9.6 & 0.4 loss at bottom \\
\hline $4 / 4 / 04$ & 89 & 11:30am & 710.0 & 712.0 & 2.0 & 2.0 & \\
\hline $4 / 4 / 04$ & 90 & $12: 45 \mathrm{pm}$ & 712.0 & 720.0 & 8.0 & 8.1 & 0.1 extra - core expansion \\
\hline $4 / 4 / 04$ & 91 & $1: 50 \mathrm{pm}$ & 720.0 & 730.0 & 10.0 & 9.3 & 0.7 loss at bottom \\
\hline $4 / 4 / 04$ & 92 & $3: 05 \mathrm{pm}$ & 730.0 & 740.0 & 10.0 & 9.5 & 0.5 loss at bottom \\
\hline $4 / 4 / 04$ & 93 & $4: 10 \mathrm{pm}$ & 740.0 & 750.0 & 10.0 & 8.9 & 1.1 loss at bottom \\
\hline 4/5/04 & 94 & $9: 15 \mathrm{am}$ & 750.0 & 759.0 & 9.0 & 2.4 & 6.6 loss at bottom \\
\hline $4 / 5 / 04$ & 95 & 10:15am & 759.0 & 765.0 & 6.0 & 3.0 & 3.0 loss at bottom \\
\hline $4 / 5 / 04$ & 96 & 11:15am & 765.0 & 770.0 & 5.0 & 5.0 & \\
\hline $4 / 5 / 04$ & 97 & 1:00pm & 770.0 & 780.0 & 10.0 & 1.0 & 9.0 loss at bottom \\
\hline $4 / 5 / 04$ & 98 & $1: 45 \mathrm{pm}$ & 780.0 & 784.0 & 4.0 & 1.1 & 2.9 loss at bottom \\
\hline $4 / 5 / 04$ & 99 & $2: 40 \mathrm{pm}$ & 784.0 & 790.0 & 6.0 & 5.2 & 0.8 loss at bottom \\
\hline $4 / 5 / 04$ & 100 & $3: 40 \mathrm{pm}$ & 790.0 & 800.0 & 10.0 & 1.7 & 8.3 loss at bottom \\
\hline 4/6/04 & 101 & $4: 45 \mathrm{pm}$ & 800.0 & 810.0 & 10.0 & 0.0 & 10.0 lost -pulled rods, only one run \\
\hline 4/7/04 & 102 & $12: 30 \mathrm{pm}$ & 810.0 & 820.0 & 10.0 & 8.2 & 1.8 loss at bottom \\
\hline $4 / 7 / 04$ & 103 & $1: 35 \mathrm{pm}$ & 820.0 & 830.0 & 10.0 & 2.3 & 7.7 loss at bottom \\
\hline $4 / 7 / 04$ & 104 & $3: 25 \mathrm{pm}$ & 830.0 & 840.0 & 10.0 & 3.2 & 6.8 loss at bottom \\
\hline 4/8/04 & 105 & 11:30am & 840.0 & 850.0 & 10.0 & 8.5 & 1.5 loss at bottom \\
\hline $4 / 8 / 04$ & 106 & $2: 00 \mathrm{pm}$ & 850.0 & 860.0 & 10.0 & 10.3 & 0.3 extra - core expansion \\
\hline $4 / 8 / 04$ & 107 & $3: 40 \mathrm{pm}$ & 860.0 & 870.0 & 10.0 & 10.2 & 0.2 extra - core expansion \\
\hline $4 / 8 / 04$ & 108 & $4: 50 \mathrm{pm}$ & 870.0 & 875.0 & 5.0 & 3.4 & 1.6 loss at bottom \\
\hline 4/9/04 & 109 & 9:50am & 875.0 & 880.0 & 5.0 & 4.2 & 0.8 loss at bottom \\
\hline $4 / 9 / 04$ & 110 & 11:25am & 880.0 & 890.0 & 10.0 & 3.5 & 6.5 loss at bottom \\
\hline $4 / 9 / 04$ & 111 & $12: 50 \mathrm{pm}$ & 890.0 & 900.0 & 10.0 & 5.0 & 5.0 loss at bottom \\
\hline $4 / 9 / 04$ & 112 & $2: 30 \mathrm{pm}$ & 900.0 & 910.0 & 10.0 & 7.4 & 2.6 loss at bottom \\
\hline $4 / 9 / 04$ & 113 & $3: 35 \mathrm{pm}$ & 910.0 & 919.5 & 9.5 & 5.3 & 4.2 loss at bottom \\
\hline $4 / 9 / 04$ & 114 & $5: 10 \mathrm{pm}$ & 919.5 & 921.5 & 2.0 & 0.0 & \\
\hline 4/10/04 & 115 & 10:15am & 921.5 & 930.0 & 8.5 & 0.0 & \\
\hline $4 / 10 / 04$ & 116 & $12: 30 \mathrm{pm}$ & 930.0 & 935.0 & 5.0 & 2.6 & 2.4 loss at bottom \\
\hline 4/10/04 & 117 & $1: 45 \mathrm{pm}$ & 935.0 & 944.0 & 9.0 & 6.2 & 2.8 loss at bottom \\
\hline $4 / 10 / 04$ & 118 & $3: 30 \mathrm{pm}$ & 944.0 & 950.0 & 6.0 & 6.3 & 0.3 extra - core expansion \\
\hline $4 / 10 / 04$ & 119 & $5: 15 \mathrm{pm}$ & 950.0 & 955.5 & 5.5 & 5.4 & 0.1 loss at bottom \\
\hline 4/11/04 & 120 & 8:40am & 955.5 & 960.0 & 4.5 & 3.4 & 1.1 loss at bottom \\
\hline $4 / 11 / 04$ & 121 & 11:10am & 960.0 & 968.0 & 8.0 & 7.9 & 0.1 loss at bottom \\
\hline $4 / 11 / 04$ & 122 & $1: 05 \mathrm{pm}$ & 968.0 & 970.5 & 2.5 & 0.0 & \\
\hline 4/11/04 & 123 & $3: 15 \mathrm{pm}$ & 970.5 & 973.0 & 2.5 & 3.4 & 0.9 extra from previous run \\
\hline $4 / 12 / 04$ & 124 & $3: 01 \mathrm{pm}$ & 973.0 & 980.0 & 7.0 & 5.3 & 1 st run on new bit \\
\hline $4 / 12 / 04$ & 125 & $6: 10 \mathrm{pm}$ & 980.0 & 985.0 & 5.0 & 3.6 & 1.4 loss at bottom \\
\hline
\end{tabular}




\begin{tabular}{|c|c|c|c|c|c|c|c|}
\hline 4/13/04 & 126 & 10:20am & 985.0 & 995.0 & 10.0 & 9.7 & 0.3 loss at bottom \\
\hline $4 / 13 / 04$ & 127 & $12: 10 \mathrm{pm}$ & 995.0 & 1000.0 & 5.0 & 3.4 & 1.6 loss at bottom \\
\hline 4/13/04 & 128 & $2: 30 \mathrm{pm}$ & 1000.0 & 1010.0 & 10.0 & 0.0 & core barrel plugged by clay \\
\hline 4/13/04 & 129 & 4:20pm & 1010.0 & 1015.0 & 5.0 & 0.0 & gravel - fell out \\
\hline 4/13/04 & 130 & $5: 45 \mathrm{pm}$ & 1015.0 & 1020.0 & 5.0 & $0.3 ?$ & plug may be from higher up \\
\hline $4 / 13 / 04$ & 131 & 7:10pm & 1020.0 & 1021.0 & 1.0 & 1.0 & full recovery \\
\hline 4/14/04 & 132 & 9:55am & 1021.0 & 1030.0 & 9.0 & 0.0 & no recovery \\
\hline $4 / 14 / 04$ & 133 & 2:10pm & 1030.0 & 1032.0 & 2.0 & 0.0 & inner barrel didn't lock \\
\hline 4/15/04 & 134 & 9:10am & 1032.0 & 1035.0 & 3.0 & 0.7 & probably from last run * \\
\hline $4 / 15 / 04$ & 135 & $10: 45 \mathrm{am}$ & 1035.0 & 1040.0 & 5.0 & 0.0 & still picking up pebbles \\
\hline $4 / 15 / 04$ & 136 & $1: 30 \mathrm{pm}$ & 1040.0 & 1045.0 & 5.0 & 0.0 & $80 \mathrm{~mm}$ pebble was blocking bit \\
\hline 4/16/04 & 137 & 8:55am & 1045.0 & 1046.0 & 1.0 & 0.0 & post stayed behind \\
\hline $4 / 16 / 04$ & 138 & 11:15am & 1046.0 & 1052.0 & 6.0 & 7.3 & recovered last on this run \\
\hline 4/16/04 & 139 & $1: 55 \mathrm{pm}$ & 1052.0 & 1060.0 & 8.0 & 4.7 & \\
\hline $4 / 16 / 04$ & 140 & $3: 50 \mathrm{pm}$ & 1060.0 & 1063.0 & 3.0 & 1.4 & \\
\hline 4/17/04 & 141 & 9:50am & 1063.0 & 1070.0 & 7.0 & 4.1 & 2.3 recovered on next run \\
\hline $4 / 17 / 04$ & 142 & $12: 20 \mathrm{pm}$ & 1070.0 & 1078.0 & 8.0 & 7.4 & 5.1 from this run $\& 2.3$ extra on last \\
\hline $4 / 17 / 04$ & 143 & $3: 30 \mathrm{pm}$ & 1078.0 & 1085.0 & 7.0 & 4.7 & fracture at top matches fracture in run 142 \\
\hline $4 / 17 / 04$ & 144 & $5: 35 \mathrm{pm}$ & 1085.0 & 1090.0 & 5.0 & 10.0 & recovered $5 \mathrm{ft}$. from run 143 (confirmed) \\
\hline 4/18/04 & 145 & $10: 25 \mathrm{am}$ & 1090.0 & 1094.5 & 4.5 & 3.2 & loss $1.2 \mathrm{ft}$., end probably never detached \\
\hline
\end{tabular}

* $0.1 \mathrm{ft}$. quartz cobble on end of inner core barrel-probable cause of core loss on last two runs. 
APPENDIX 3. Hope Plantation sampling log, showing sample type, depth, individual who sampled the core, and medium for which it was sampled (i.e. microfossil, hydrogeology).

\section{Hope Plantation - Sampling Log}

\begin{tabular}{|c|c|c|c|c|c|c|}
\hline Date & Sample Type & Top Depth & $\begin{array}{c}\text { Bottom } \\
\text { Depth }\end{array}$ & Taken By & Taken For & $\begin{array}{l}\text { Purpose (nannos, } \\
\text { dinos, etc.) }\end{array}$ \\
\hline 3/13/04 & Half core & 47.7 & 48.0 & Durand & Edwards & Dinoflagellates \\
\hline $3 / 13 / 04$ & Half core & 96.7 & 97.0 & Durand & Edwards & Dinoflagellates \\
\hline $3 / 13 / 04$ & Half core & 117.0 & 117.3 & Durand & Edwards & Dinoflagellates \\
\hline $3 / 13 / 04$ & Spatula & 96.5 & . & Self-Trail & Self-Trail & Nannofossils \\
\hline $3 / 13 / 04$ & Spatula & 102.0 & . & Self-Trail & Self-Trail & Nannofossils \\
\hline $3 / 13 / 04$ & Spatula & 107.0 & . & Self-Trail & Self-Trail & Nannofossils \\
\hline $3 / 13 / 04$ & Spatula & 116.8 & . & Self-Trail & Self-Trail & Nannofossils \\
\hline $3 / 14 / 04$ & Half core & 133.0 & 133.3 & Durand & Edwards & Dinoflagellates \\
\hline $3 / 14 / 04$ & Spatula & 122.0 & . & Self-Trail & Self-Trail & Nannofossils \\
\hline $3 / 14 / 04$ & Spatula & 127.0 & . & Self-Trail & Self-Trail & Nannofossils \\
\hline $3 / 14 / 04$ & Spatula & 132.5 & . & Self-Trail & Self-Trail & Nannofossils \\
\hline $3 / 14 / 04$ & Spatula & 137.0 & . & Self-Trail & Self-Trail & Nannofossils \\
\hline $3 / 14 / 04$ & Spatula & 142.0 & . & Self-Trail & Self-Trail & Nannofossils \\
\hline $3 / 14 / 04$ & Whole core & 164.0 & 164.2 & Wrege & Wrege & Water \\
\hline $3 / 14 / 04$ & Half core & 165.6 & 166.0 & Durand & Edwards & Dinoflagellates \\
\hline $3 / 14 / 04$ & Half core & 185.6 & 185.8 & Durand & Edwards & Dinoflagellates \\
\hline $3 / 14 / 04$ & Spatula & 159.0 & . & Self-Trail & Self-Trail & Nannofossils \\
\hline $3 / 14 / 04$ & Spatula & 154.0 & . & Self-Trail & Self-Trail & Nannofossils \\
\hline $3 / 14 / 04$ & Spatula & 147.1 & . & Self-Trail & Self-Trail & Nannofossils \\
\hline $3 / 14 / 04$ & Half core & 190.7 & 190.9 & Durand & Edwards & Dinoflagellates \\
\hline $3 / 15 / 04$ & Aspirate $.05 \mathrm{ml}$ & 260.3 & 260.3 & Wrege & Bratton & Salinity \\
\hline $3 / 15 / 04$ & Half core & 261.2 & 261.4 & Wrege & Wrege & $\%$ Fines \\
\hline $3 / 16 / 04$ & Half core & 280.5 & 280.8 & Durand & Edwards/Christopher & Dinoflagellates/Pollen \\
\hline 3/17/04 & Spatula & 280.8 & . & Self-Trail & Self-Trail & Nannofossils \\
\hline $3 / 17 / 04$ & Spatula & 284.4 & . & Self-Trail & Self-Trail & Nannofossils \\
\hline $3 / 17 / 04$ & Spatula & 291.0 & . & Self-Trail & Self-Trail & Nannofossils \\
\hline $3 / 17 / 04$ & Half core & 306.0 & 306.3 & Durand & Edwards/Christopher & Dinoflagellates/Pollen \\
\hline 3/18/04 & Spatula & 309.1 & . & Self-Trail & Self-Trail & Nannofossils \\
\hline 3/19/04 & Spatula & 383.3 & . & Self-Trail & Self-Trail & Nannofossils \\
\hline $3 / 20 / 04$ & Half core & 500.6 & 500.9 & Self-Trail & Edwards/Christopher & Dinoflagellates/Pollen \\
\hline $3 / 20 / 04$ & Spatula & 518.1 & . & Seefelt & Self-Trail & Nannofossils \\
\hline $3 / 20 / 04$ & Half core & 157.0 & 157.3 & Self-Trail & Edwards & Dinoflagellates/Pollen \\
\hline $3 / 20 / 04$ & Half core & 148.0 & 148.3 & Self-Trail & Edwards & Dinoflagellates/Pollen \\
\hline $3 / 20 / 04$ & Half core & 110.5 & 110.7 & Self-Trail & Edwards & Dinoflagellates/Pollen \\
\hline $3 / 21 / 04$ & Half core & 531.0 & 531.2 & Self-Trail & Edwards & Dinoflagellates/Pollen \\
\hline $3 / 21 / 04$ & Half core & 571.9 & 572.1 & Self-Trail & Edwards/Christopher & Dinoflagellates/Pollen \\
\hline $3 / 22 / 04$ & Half core & 613.3 & 613.5 & Self-Trail & Christopher & Pollen \\
\hline
\end{tabular}




\begin{tabular}{|c|c|c|c|c|c|c|}
\hline $3 / 22 / 04$ & Half core & 620.3 & 620.5 & Self-Trail & Edwards/Christopher & Dinoflagellates/Pollen \\
\hline $3 / 22 / 04$ & Half core & 625.0 & 625.2 & Self-Trail & Edwards/Christopher & Dinoflagellates/Pollen \\
\hline $3 / 22 / 04$ & Spatula & 620.9 & . & Self-Trail & Self-Trail & Nannofossils \\
\hline $3 / 22 / 04$ & Half core & 640.8 & 641.0 & Self-Trail & Edwards/Christopher & Dinoflagellates/Pollen \\
\hline $3 / 23 / 04$ & Spatula & 646.4 & . & Self-Trail & Self-Trail & Nannofossils \\
\hline $3 / 23 / 04$ & Spatula & 646.5 & . & Self-Trail & Self-Trail & Nannofossils \\
\hline $3 / 23 / 04$ & Half core & 657.9 & 658.1 & Seefelt & Edwards/Christopher & Dinoflagellates/Pollen \\
\hline $3 / 23 / 04$ & Spatula & 646.6 & . & Seefelt & Self-Trail & Nannofossils \\
\hline $3 / 23 / 04$ & Spatula & 648.6 & . & Seefelt & Self-Trail & Nannofossils \\
\hline $3 / 23 / 04$ & Spatula & 653.4 & . & Seefelt & Self-Trail & Nannofossils \\
\hline $3 / 23 / 04$ & Spatula & 656.4 & . & Seefelt & Self-Trail & Nannofossils \\
\hline $3 / 23 / 04$ & Spatula & 651.3 & . & Seefelt & Self-Trail & Nannofossils \\
\hline $3 / 23 / 04$ & Spot core & 659.8 & 659.9 & Self-Trail & Self-Trail & Nannofossils etc? \\
\hline $4 / 4 / 04$ & Spatula & 677.0 & . & Self-Trail & Self-Trail & Nannofossils \\
\hline $4 / 4 / 04$ & Spatula & 686.3 & . & Self-Trail & Self-Trail & Nannofossils \\
\hline $4 / 4 / 04$ & Spatula & 696.0 & . & Self-Trail & Self-Trail & Nannofossils \\
\hline $4 / 4 / 04$ & Spatula & 702.0 & . & Self-Trail & Self-Trail & Nannofossils \\
\hline $4 / 5 / 04$ & Spatula & 714.7 & . & Self-Trail & Self-Trail & Nannofossils \\
\hline $4 / 5 / 04$ & Half core & 662.2 & 662.5 & Self-Trail & Edwards/Christopher & Dinoflagellates/Pollen \\
\hline $4 / 5 / 04$ & Half core & 687.4 & 687.7 & Self-Trail & Edwards/Christopher & Dinoflagellates/Pollen \\
\hline $4 / 5 / 04$ & Half core & 714.6 & 714.9 & Self-Trail & Edwards/Christopher & Dinoflagellates/Pollen \\
\hline $4 / 5 / 04$ & Half core & 736.5 & 736.8 & Self-Trail & Edwards/Christopher & Dinoflagellates/Pollen \\
\hline $4 / 5 / 04$ & Half core & 746.7 & 747.0 & Self-Trail & Edwards/Christopher & Dinoflagellates/Pollen \\
\hline $4 / 5 / 04$ & Spatula & 717.5 & - & Self-Trail & Self-Trail & Nannofossils \\
\hline $4 / 6 / 04$ & Half core & 122.7 & 123.0 & Prowell & Edwards/Christopher & Dinoflagellates/Pollen \\
\hline $4 / 6 / 04$ & Half core & 127.0 & 127.2 & Prowell & Edwards/Christopher & Dinoflagellates/Pollen \\
\hline $4 / 6 / 04$ & Half core & 180.2 & 180.4 & Prowell & Edwards/Christopher & Dinoflagellates/Pollen \\
\hline $4 / 6 / 04$ & Half core & 223.5 & 223.6 & Prowell & Edwards/Christopher & Dinoflagellates/Pollen \\
\hline $4 / 6 / 04$ & Half core & 277.2 & 277.4 & Prowell & Edwards/Christopher & Dinoflagellates/Pollen \\
\hline $4 / 6 / 04$ & Half core & 330.4 & 330.6 & Prowell & Edwards/Christopher & Dinoflagellates/Pollen \\
\hline $4 / 6 / 04$ & Half core & 341.3 & 341.5 & Prowell & Edwards/Christopher & Dinoflagellates/Pollen \\
\hline $4 / 6 / 04$ & Half core & 351.3 & 351.4 & Prowell & Edwards/Christopher & Dinoflagellates/Pollen \\
\hline 4/7/04 & Half core & 810.3 & 810.5 & Prowell & Christopher & Pollen \\
\hline 4/8/04 & Spatula & 665.9 & . & Harris & Harris & Strontium \\
\hline $4 / 8 / 04$ & Spatula & 668.6 & . & Harris & Harris & Strontium \\
\hline $4 / 8 / 04$ & Spatula & 674.9 & . & Harris & Harris & Strontium \\
\hline $4 / 8 / 04$ & Spatula & 679.2 & . & Harris & Harris & Strontium \\
\hline $4 / 8 / 04$ & Spatula & 682.5 & . & Harris & Harris & Strontium \\
\hline $4 / 8 / 04$ & Spatula & 685.9 & . & Harris & Harris & Strontium \\
\hline $4 / 8 / 04$ & Spatula & 688.6 & . & Harris & Harris & Strontium \\
\hline $4 / 8 / 04$ & Spatula & 692.0 & . & Harris & Harris & Strontium \\
\hline $4 / 8 / 04$ & Spatula & 698.0 & . & Harris & Harris & Strontium \\
\hline $4 / 8 / 04$ & Spatula & 708.4 & . & Harris & Harris & Strontium \\
\hline $4 / 8 / 04$ & Spatula & 712.0 & . & Harris & Harris & Strontium \\
\hline $4 / 8 / 04$ & Spatula & 718.7 & . & Harris & Harris & Strontium \\
\hline $4 / 8 / 04$ & Spatula & 721.7 & . & Harris & Harris & Strontium \\
\hline 4/11/04 & Half core & 882.0 & 882.2 & Self-Trail & Christopher & Pollen \\
\hline
\end{tabular}




$\begin{array}{lcccccc}\text { 4/11/04 } & \text { Half core } & 882.2 & 882.4 & \text { Prowell } & \text { Christopher } & \text { Pollen } \\ 4 / 11 / 04 & \text { Half core } & 966.2 & 966.4 & \text { Self-Trail } & \text { Christopher } & \text { Pollen } \\ 4 / 11 / 04 & \text { Spatula } & 733.0 & \cdot & \text { Self-Trail } & \text { Self-Trail } & \text { Nannofossils } \\ 4 / 11 / 04 & \text { Spatula } & 737.9 & \cdot & \text { Self-Trail } & \text { Self-Trail } & \text { Nannofossils } \\ \text { 4/11/04 } & \text { Spatula } & 746.0 & \cdot & \text { Self-Trail } & \text { Self-Trail } & \text { Nannofossils } \\ \mathbf{4 / 1 2 / 0 4} & \text { Half core } & 732.0 & 732.2 & \text { Self-Trail } & \text { Edwards/Christopher } & \text { Dinoflagellates/Pollen } \\ \text { 4/12/04 } & \text { Half core } & 745.6 & 745.8 & \text { Self-Trail } & \text { Edwards/Christopher } & \text { Dinoflagellates/Pollen }\end{array}$




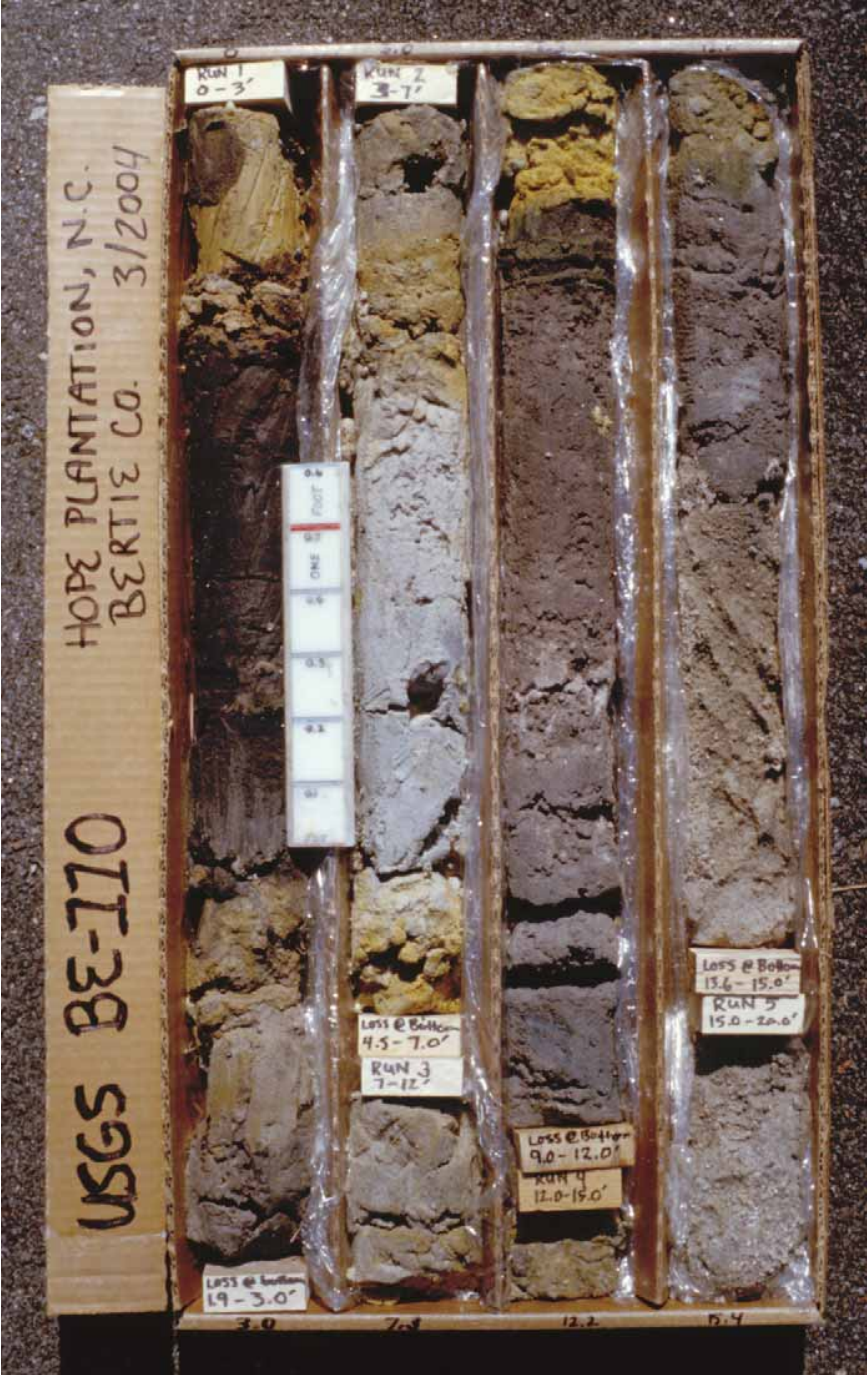




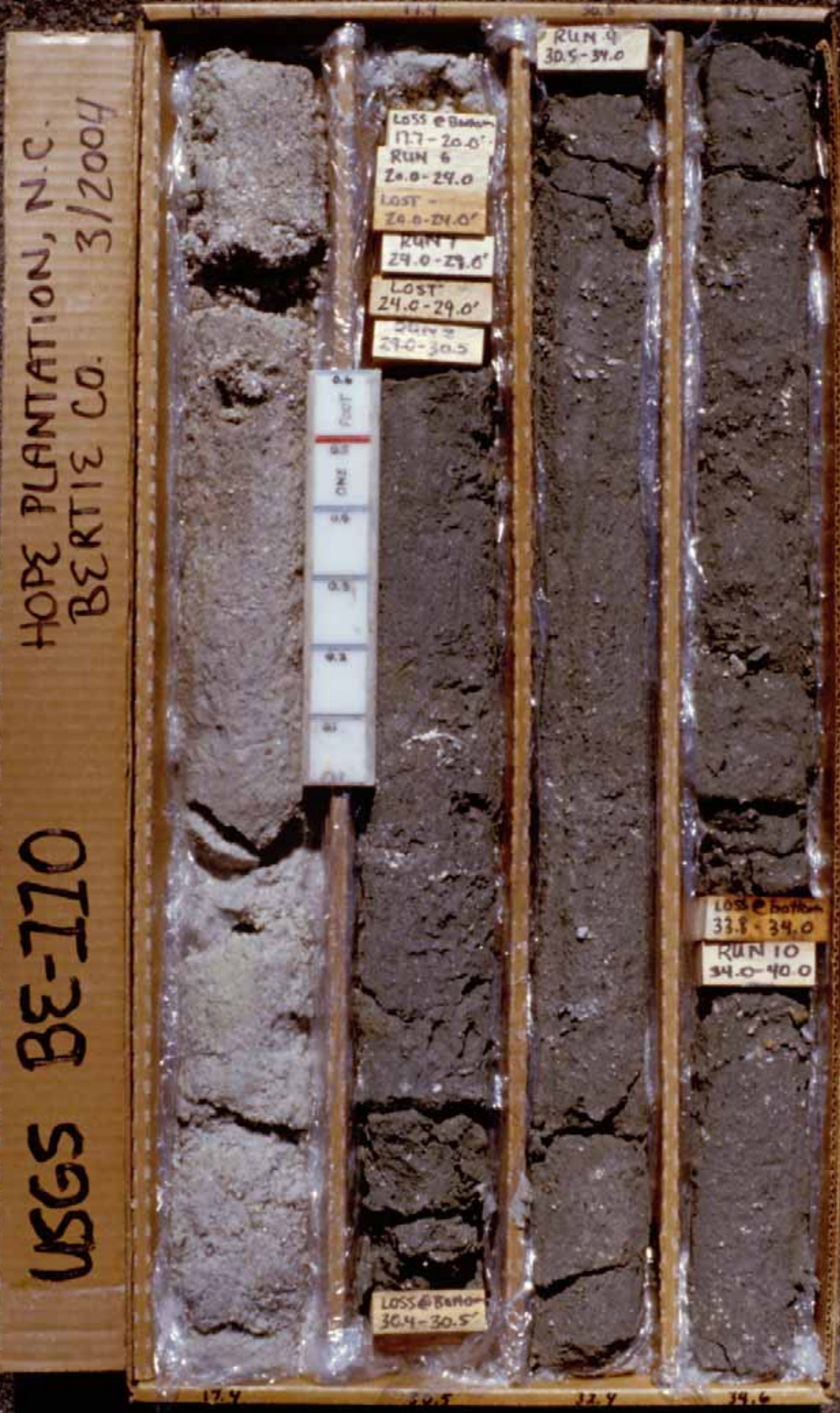




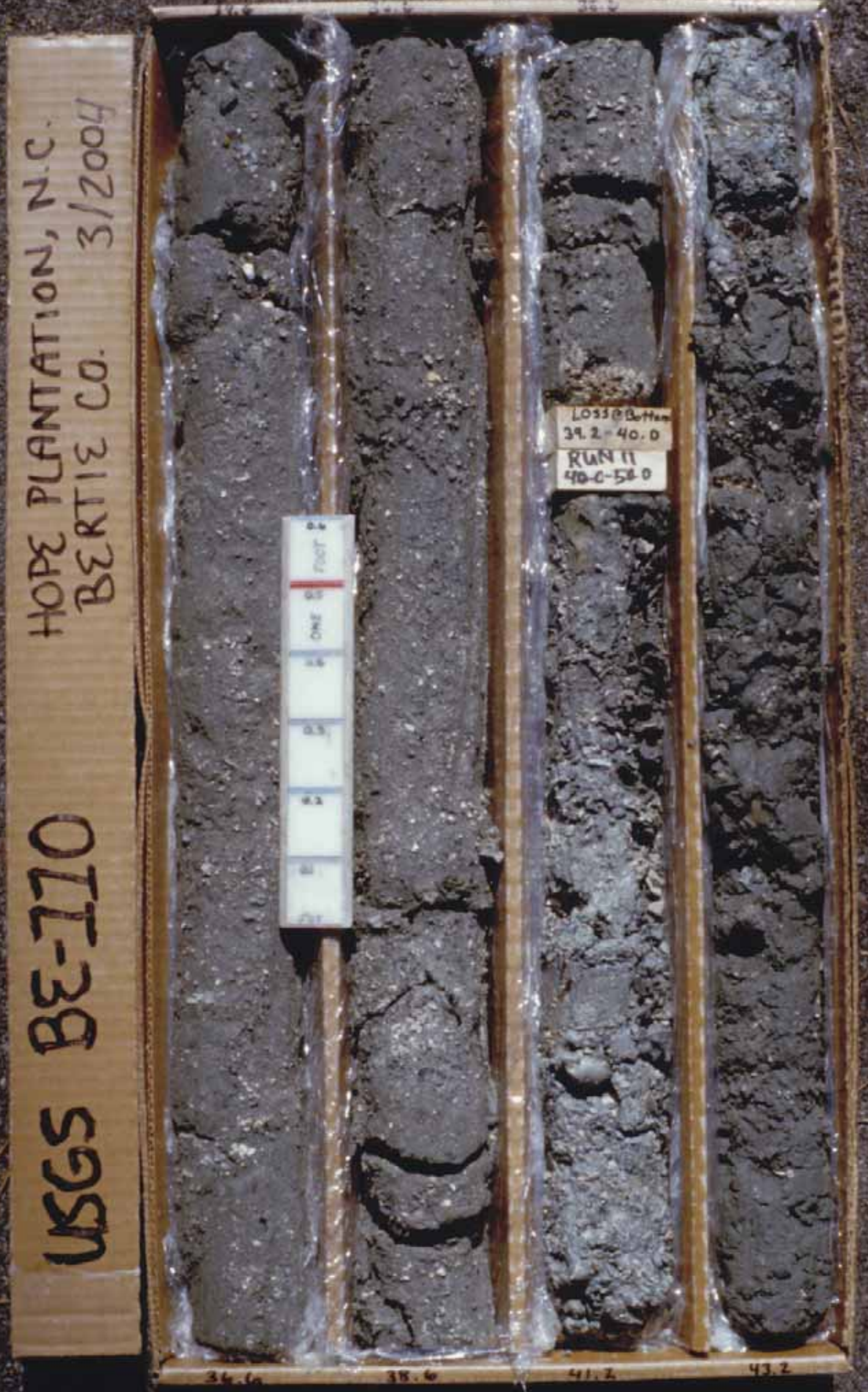




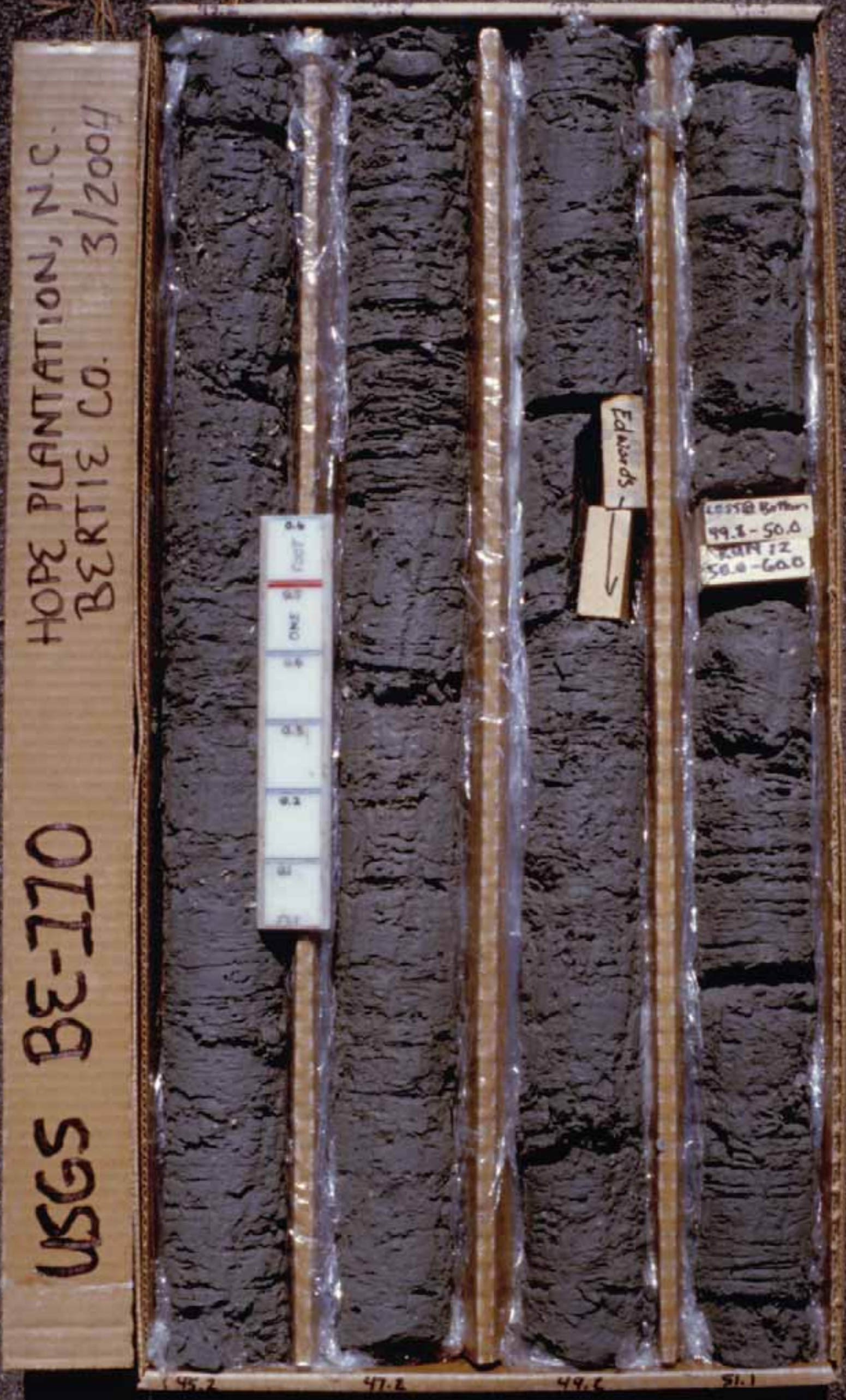




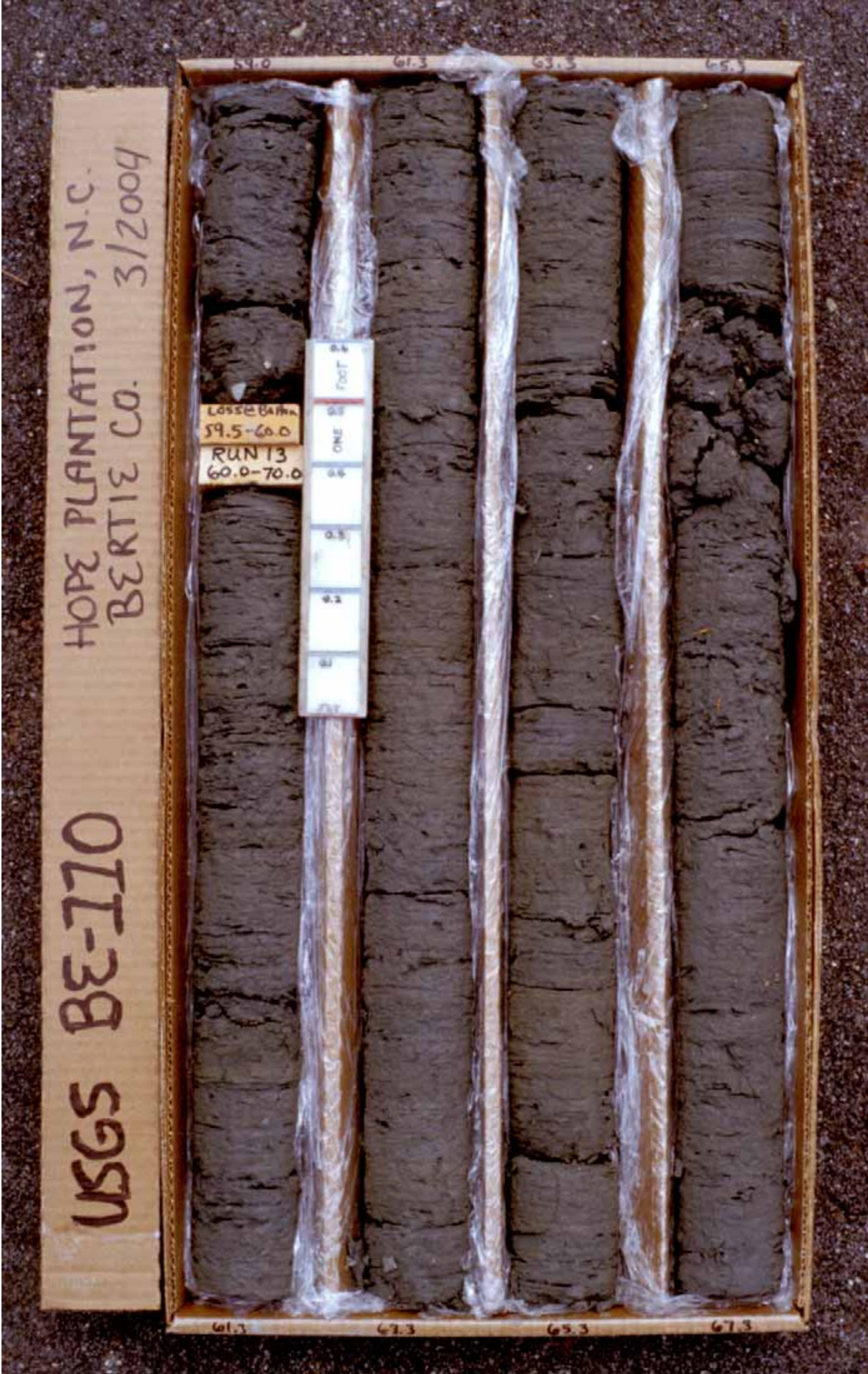




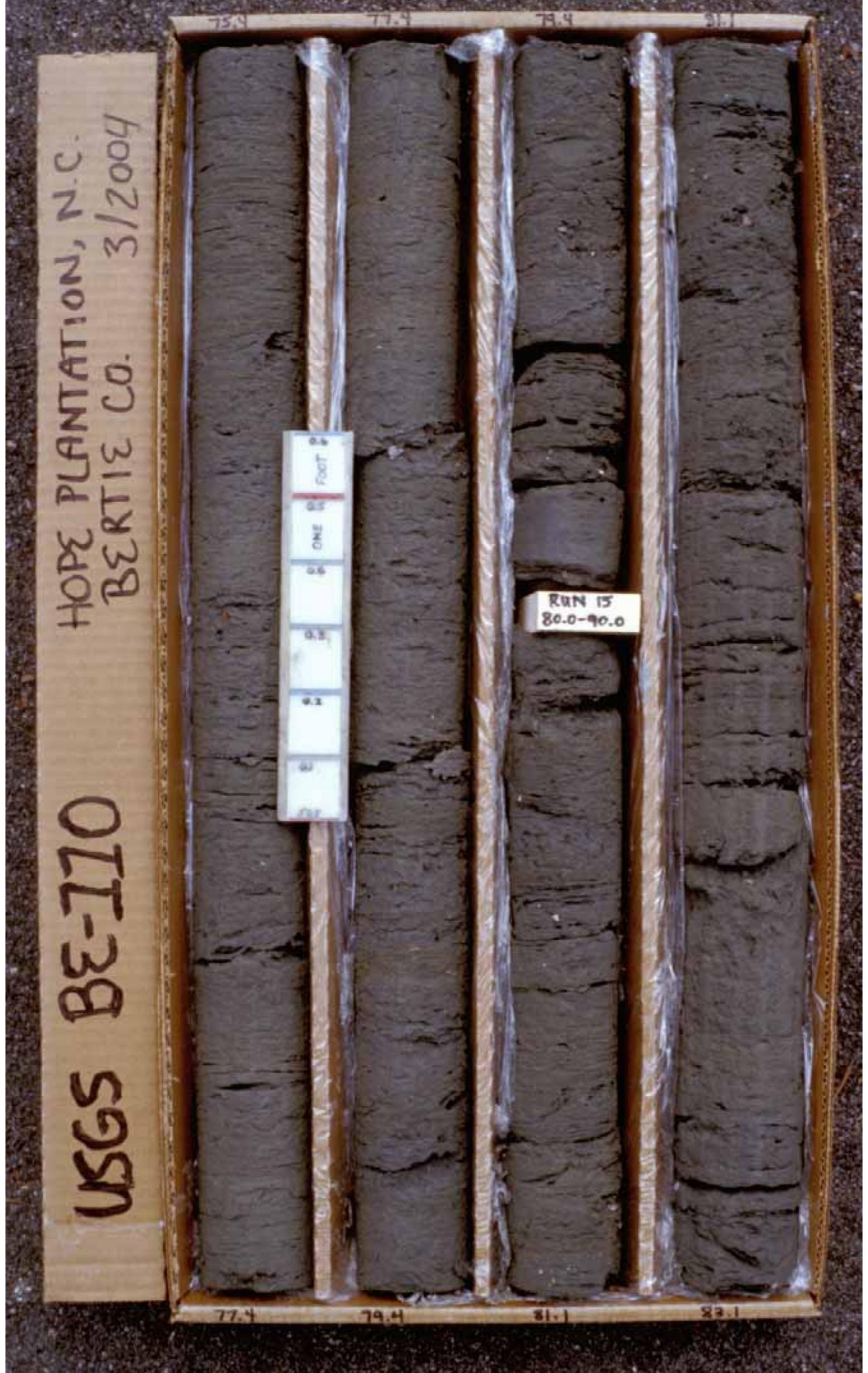




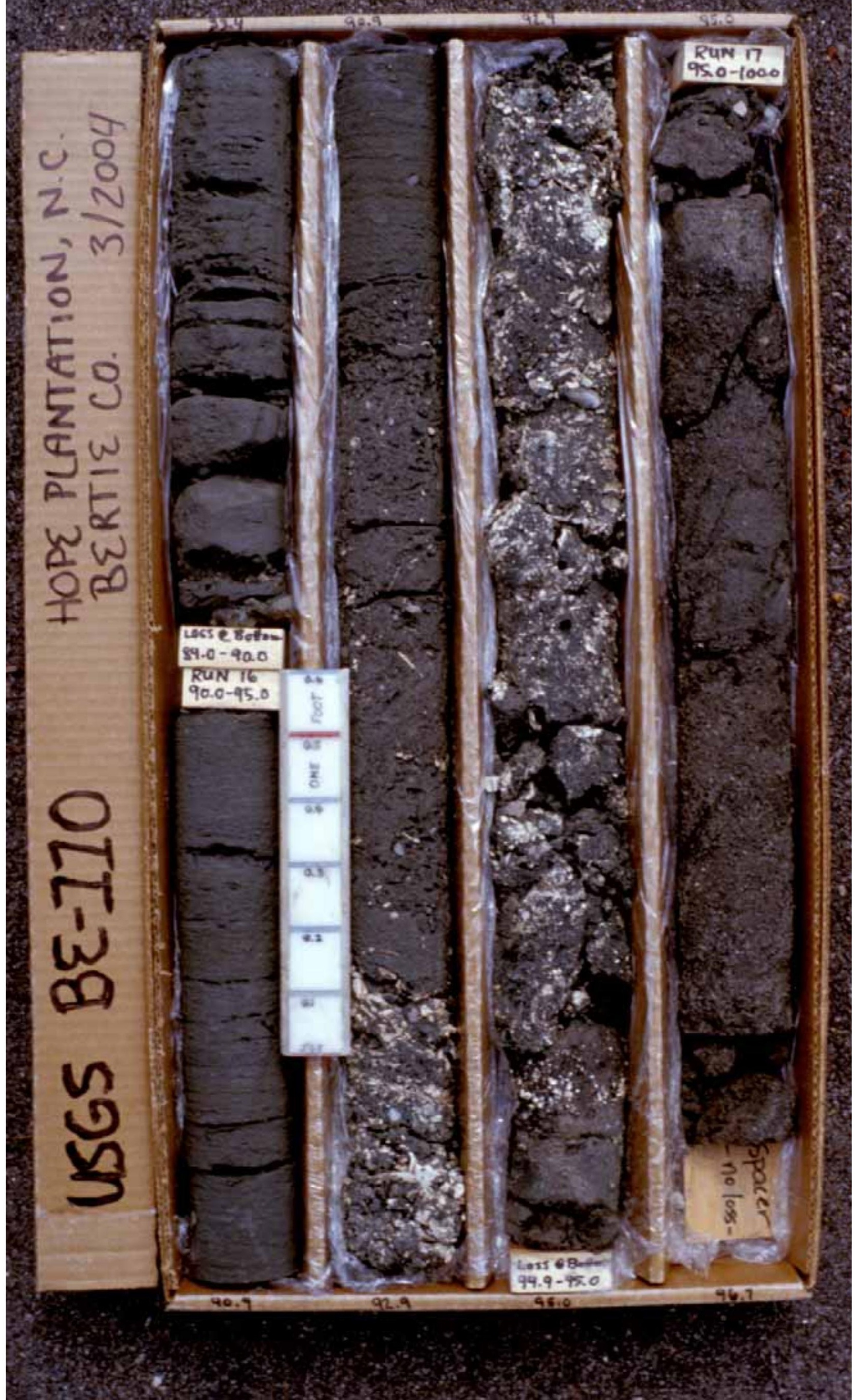




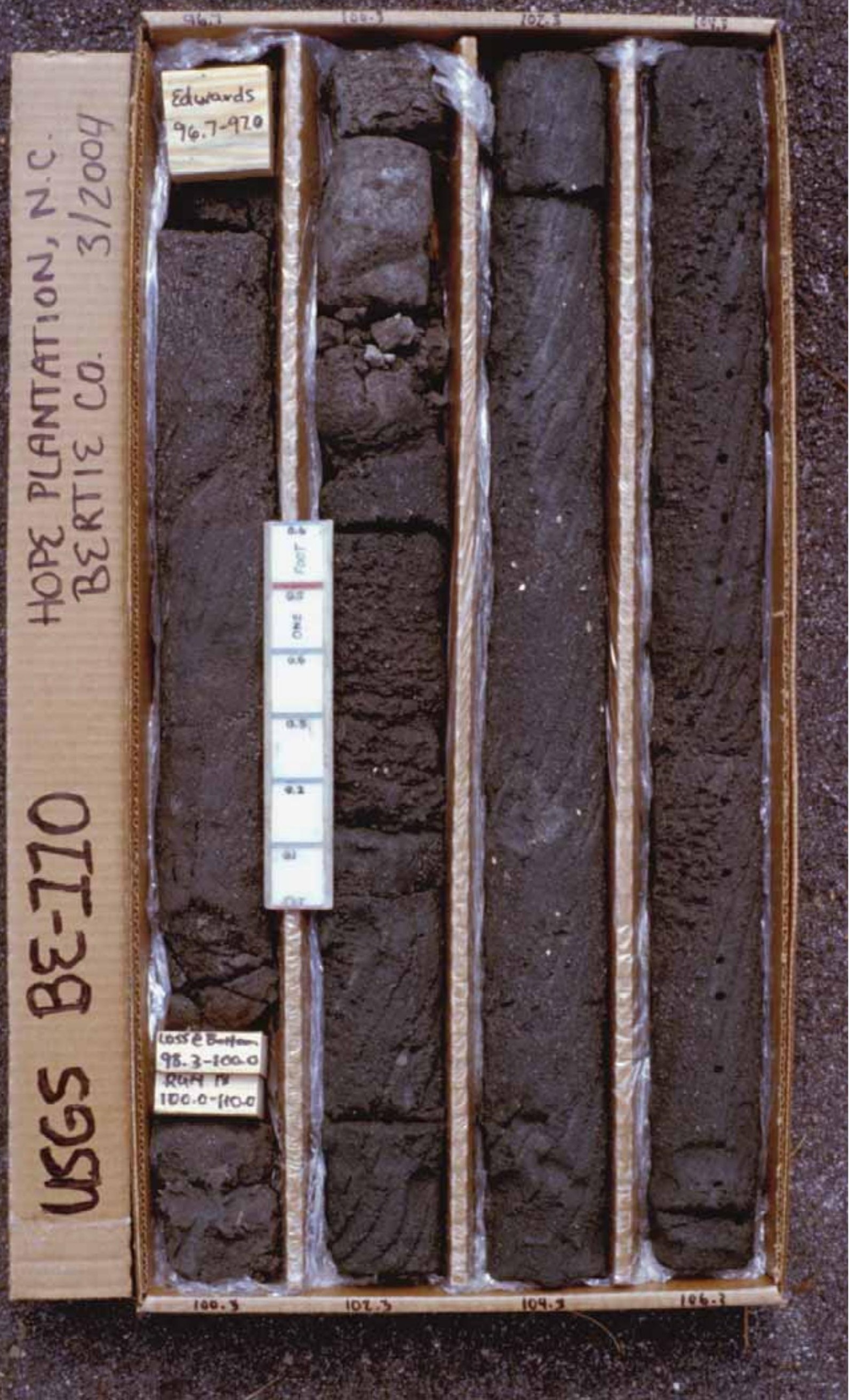




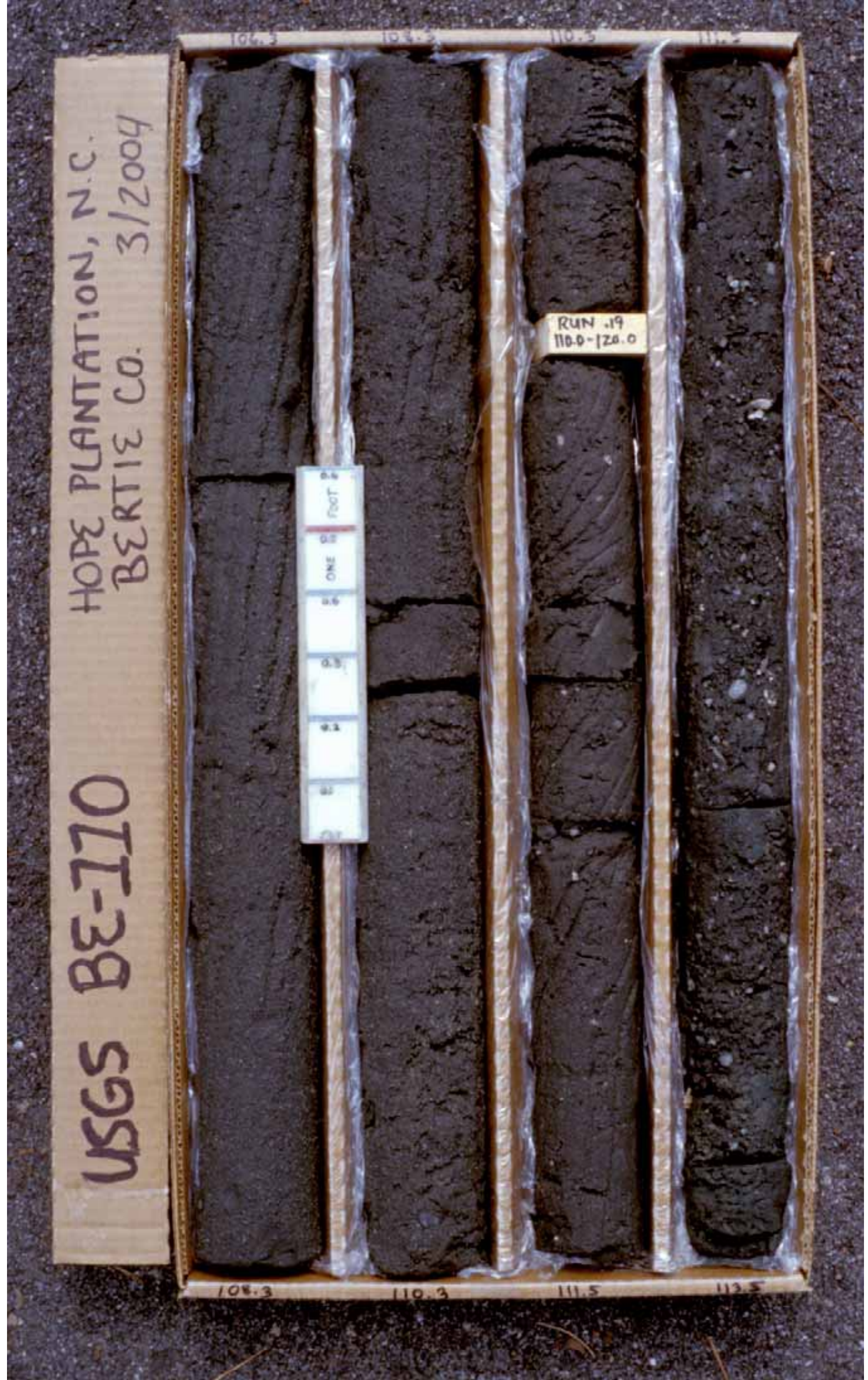




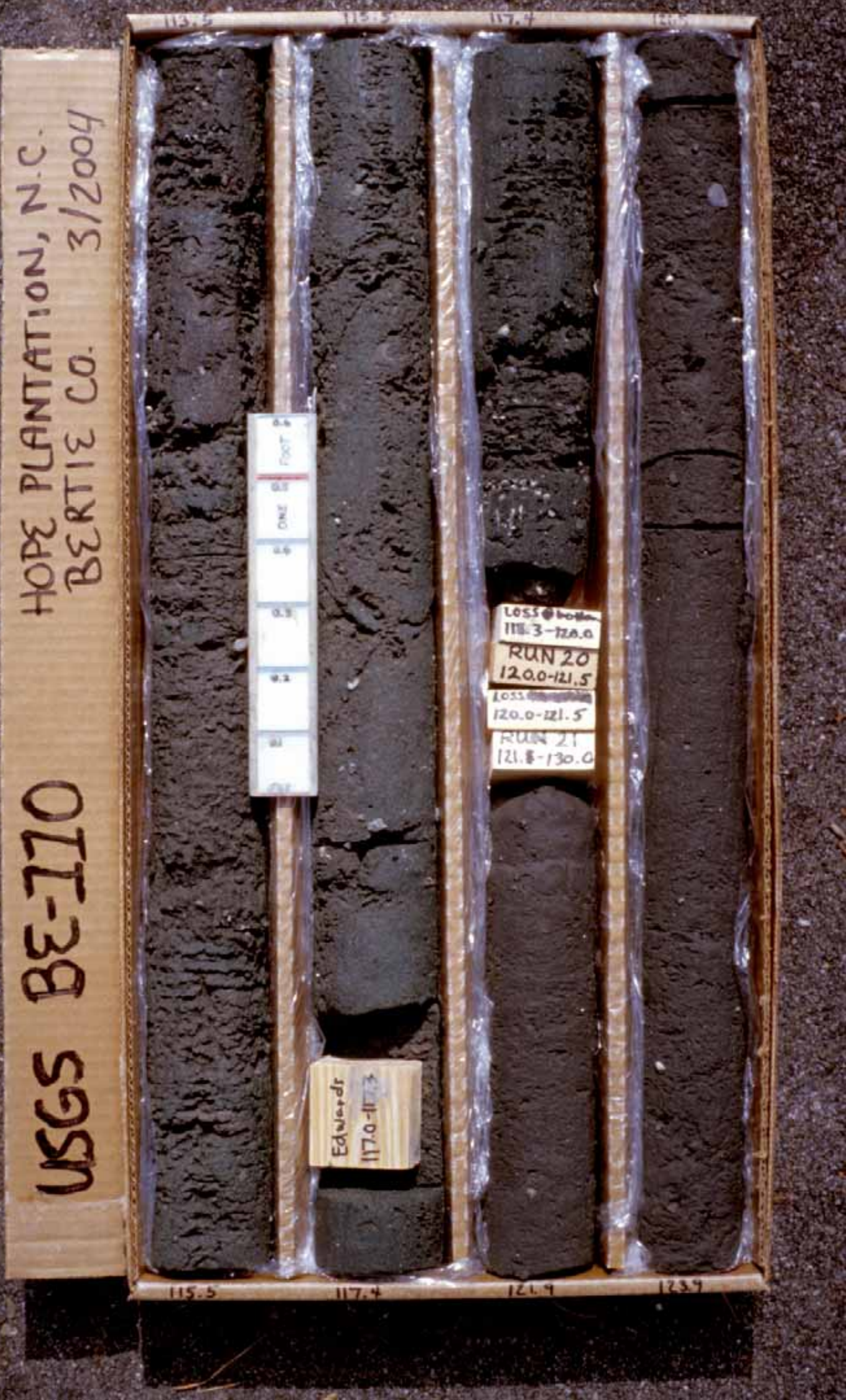




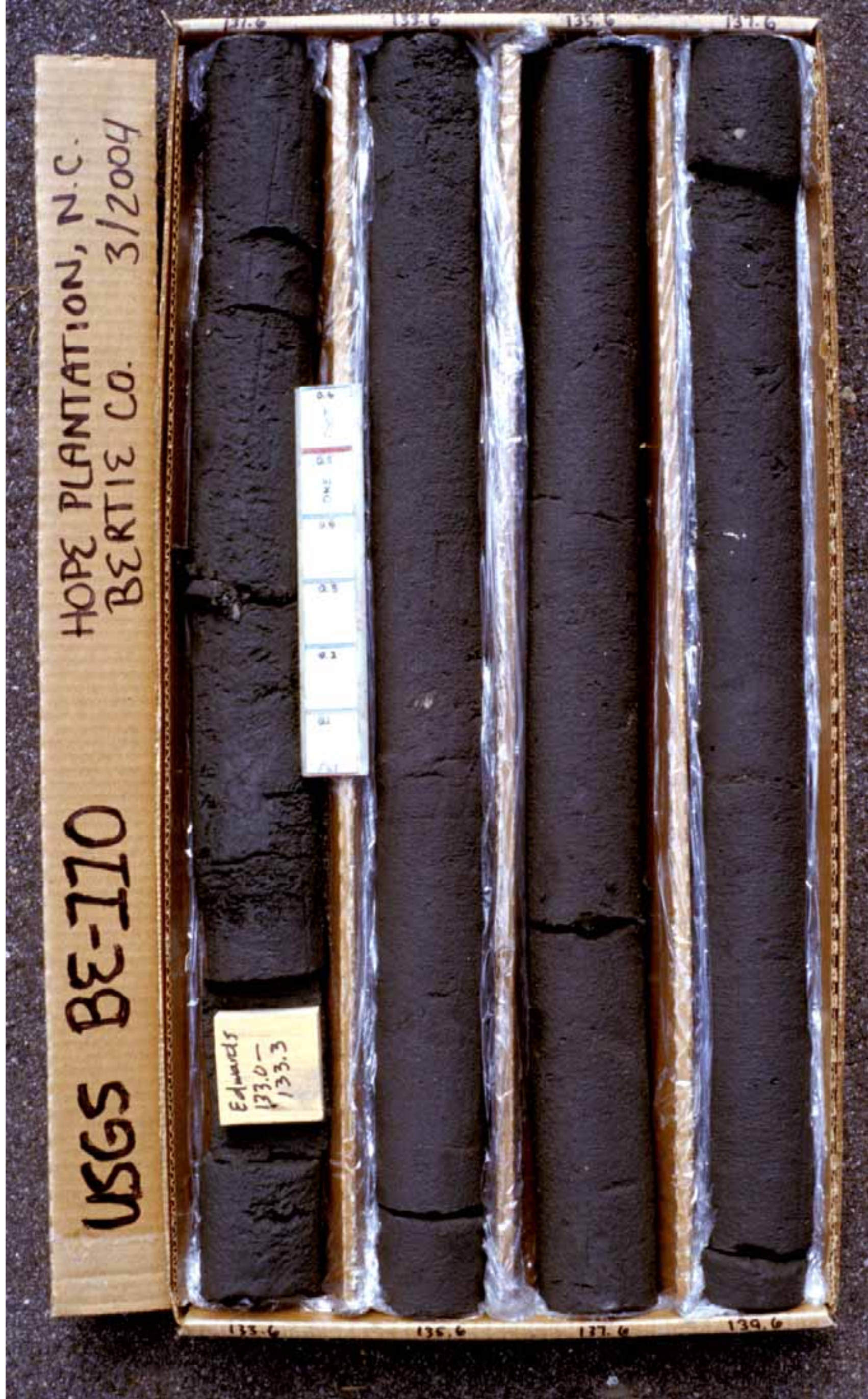




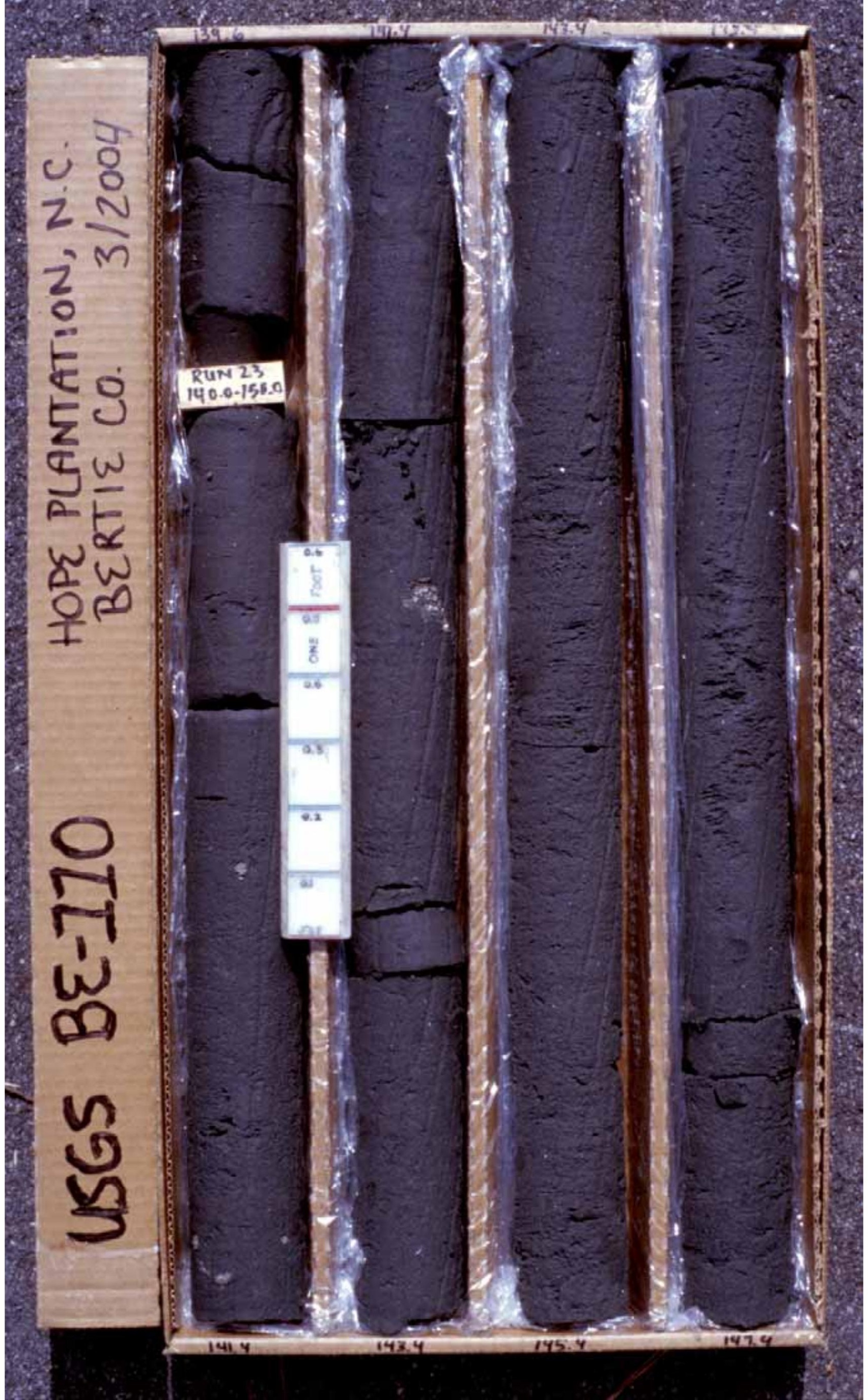




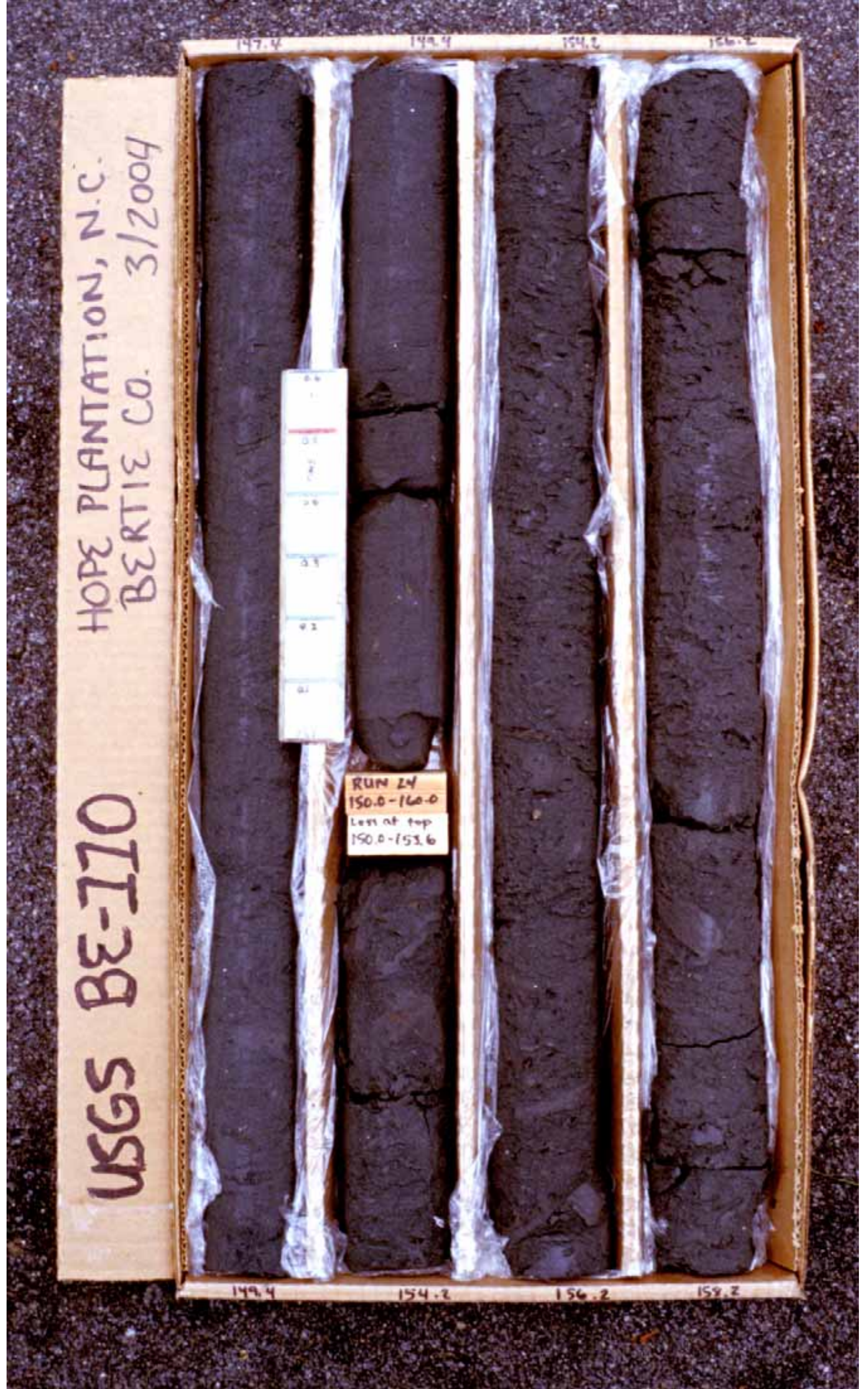




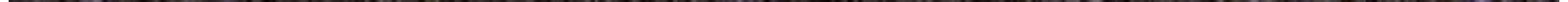




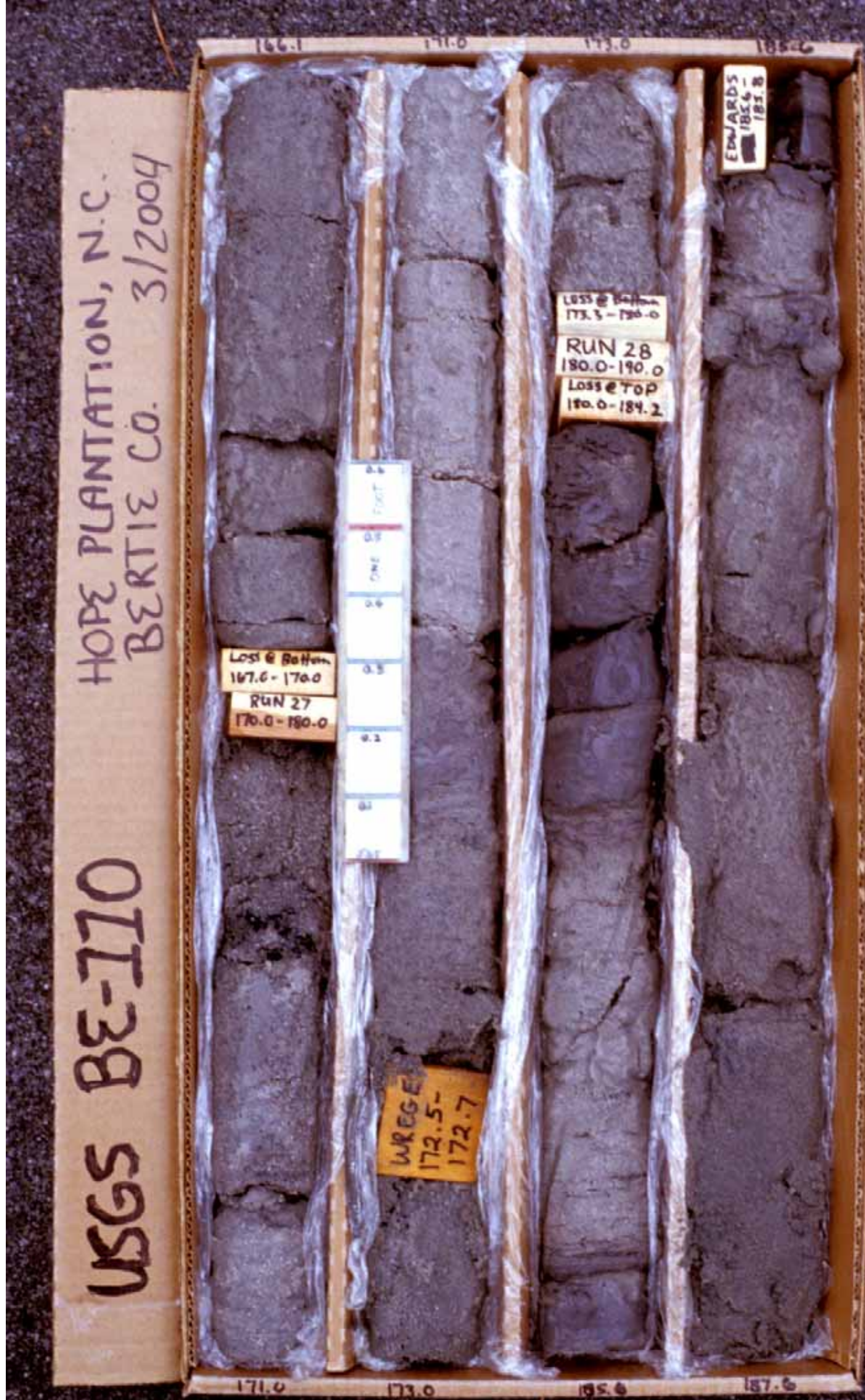




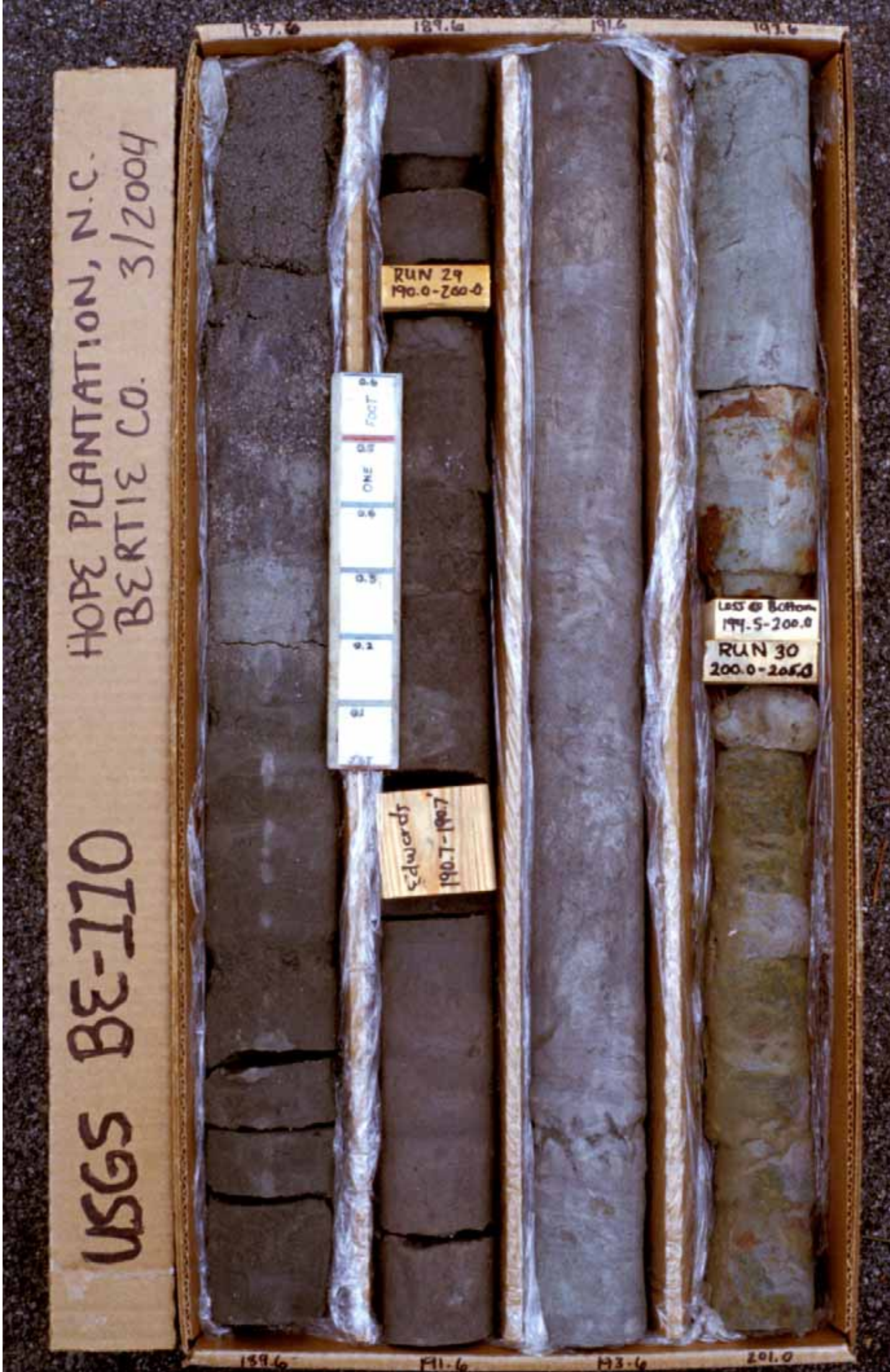




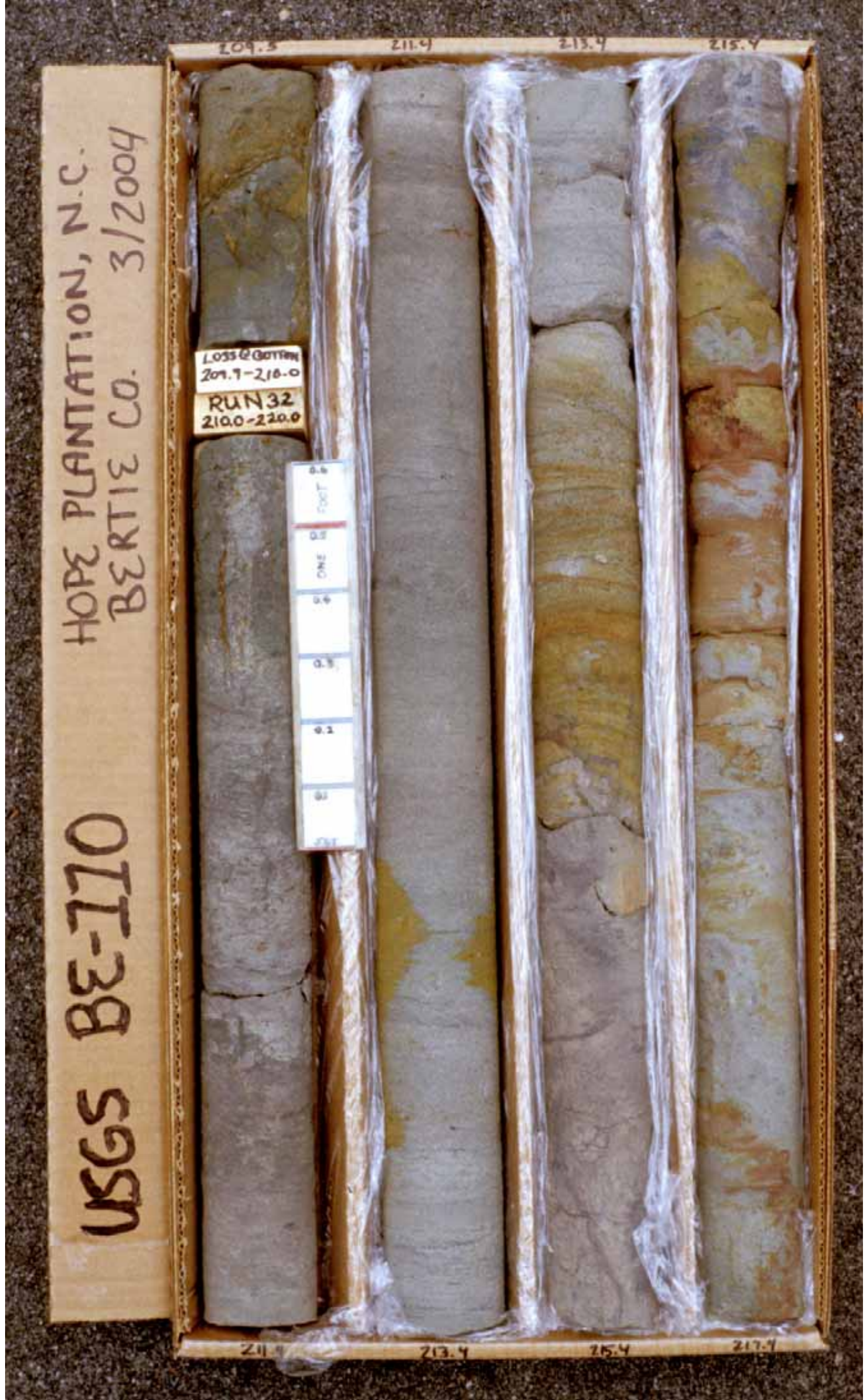




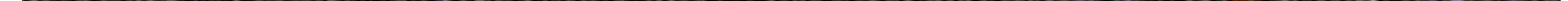




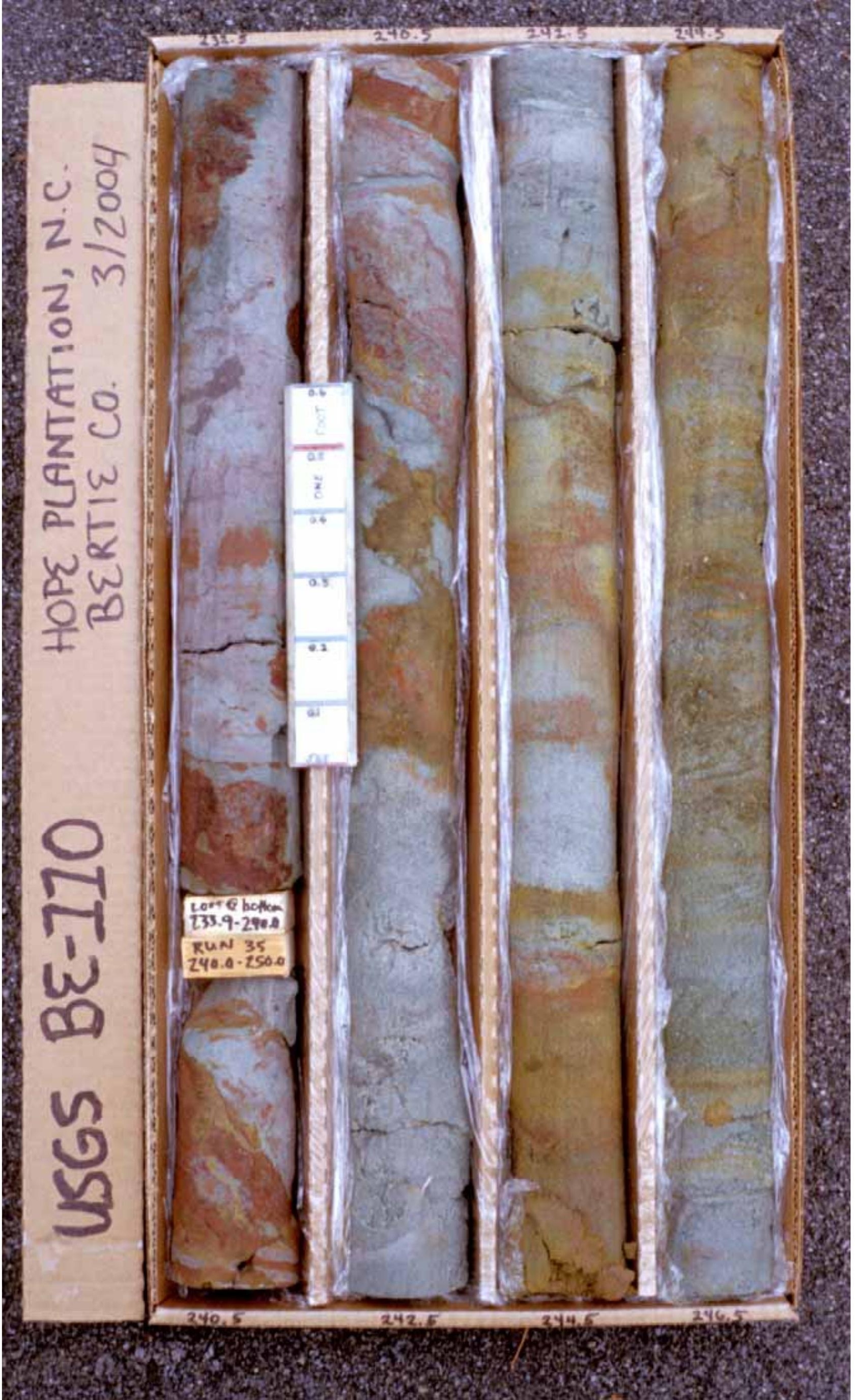




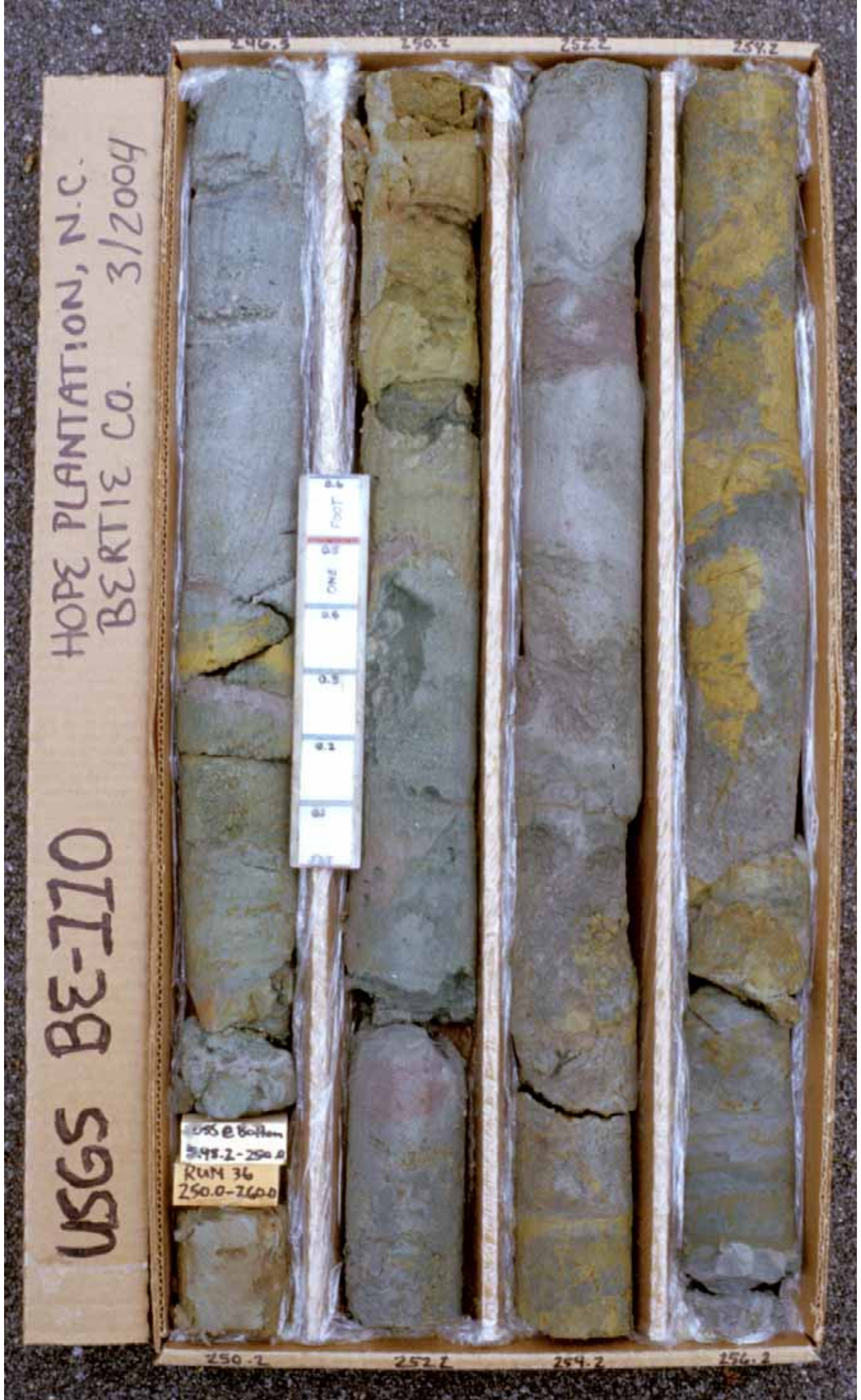




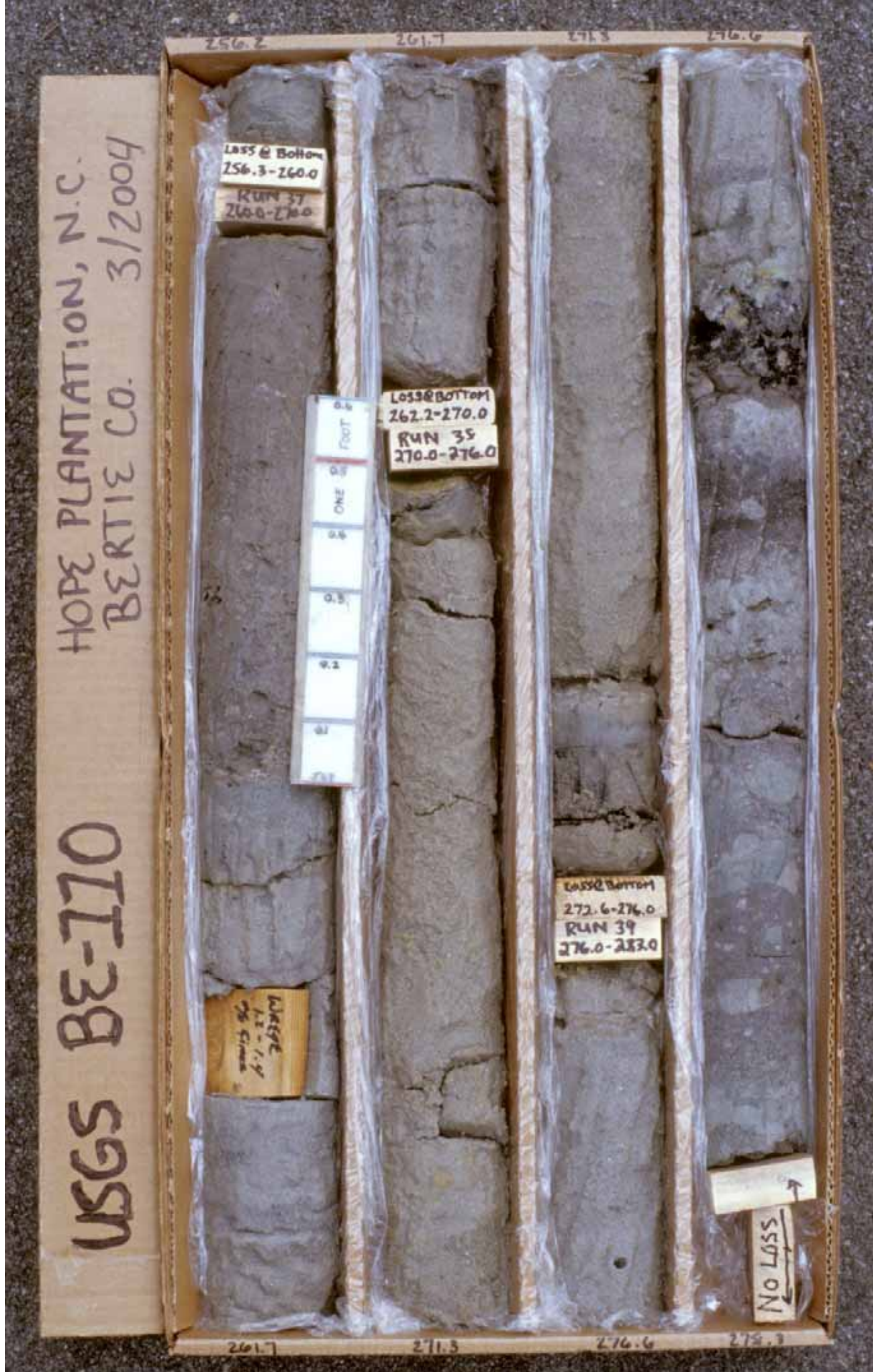




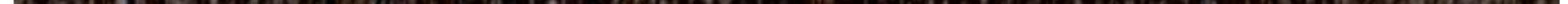




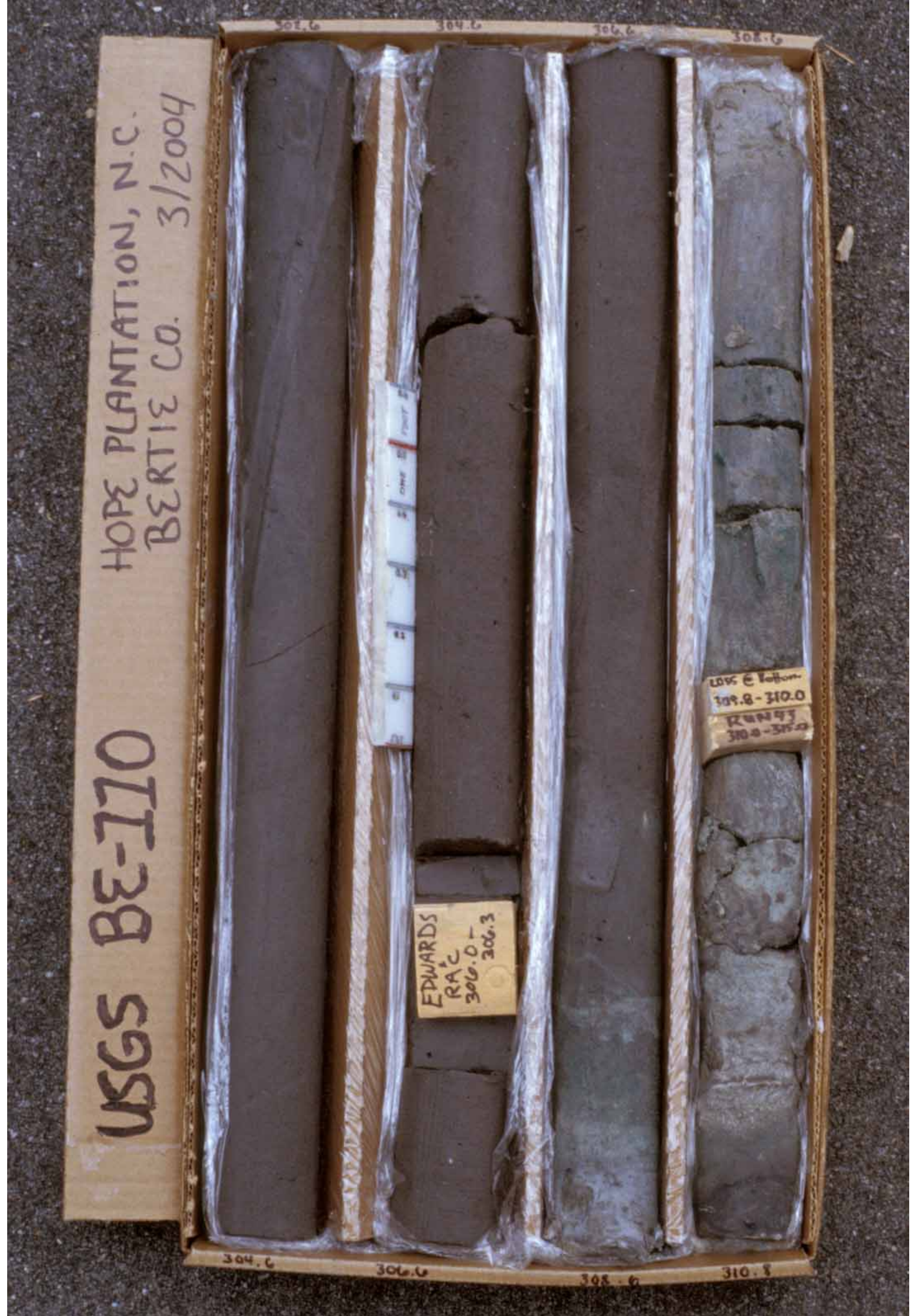




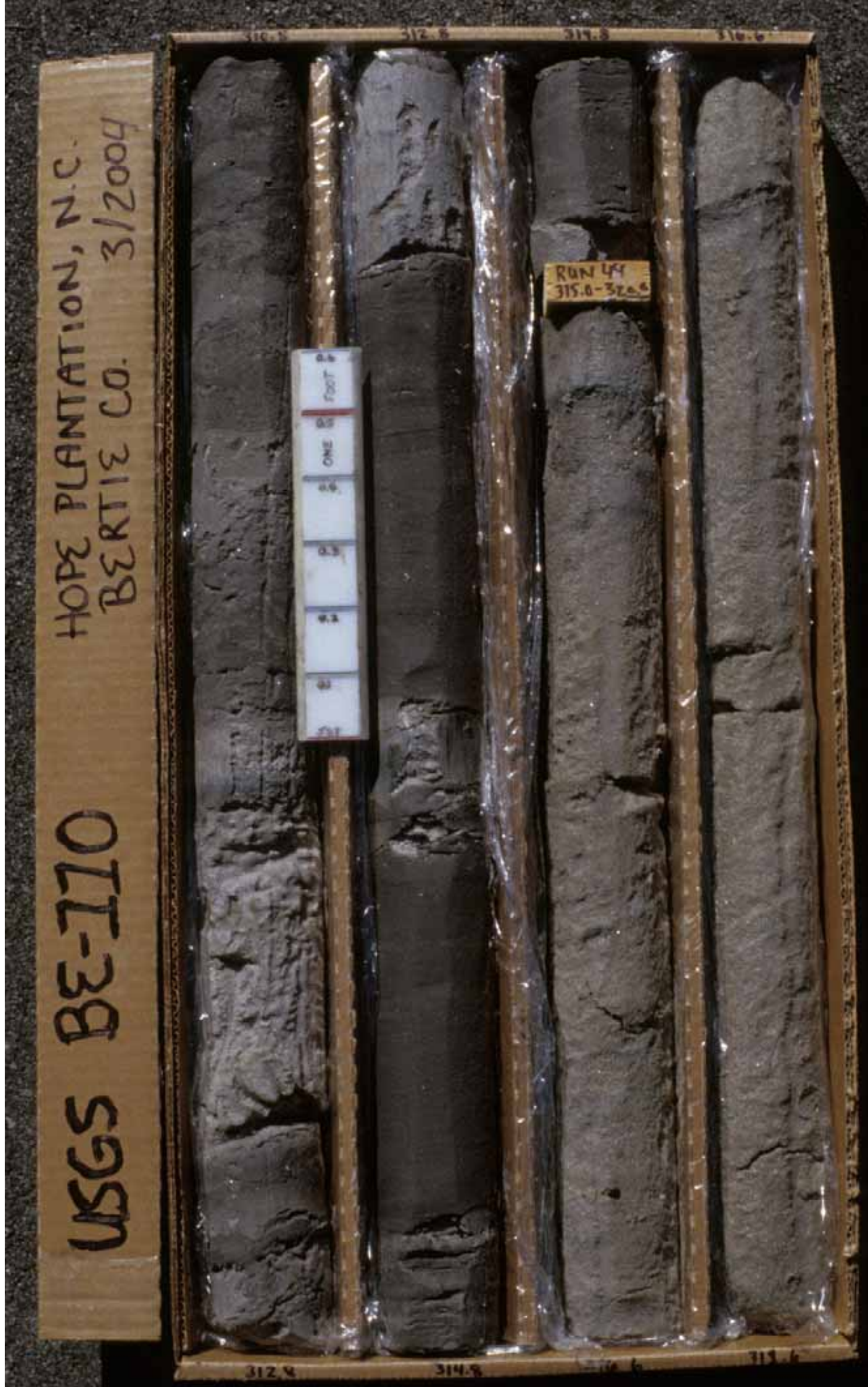




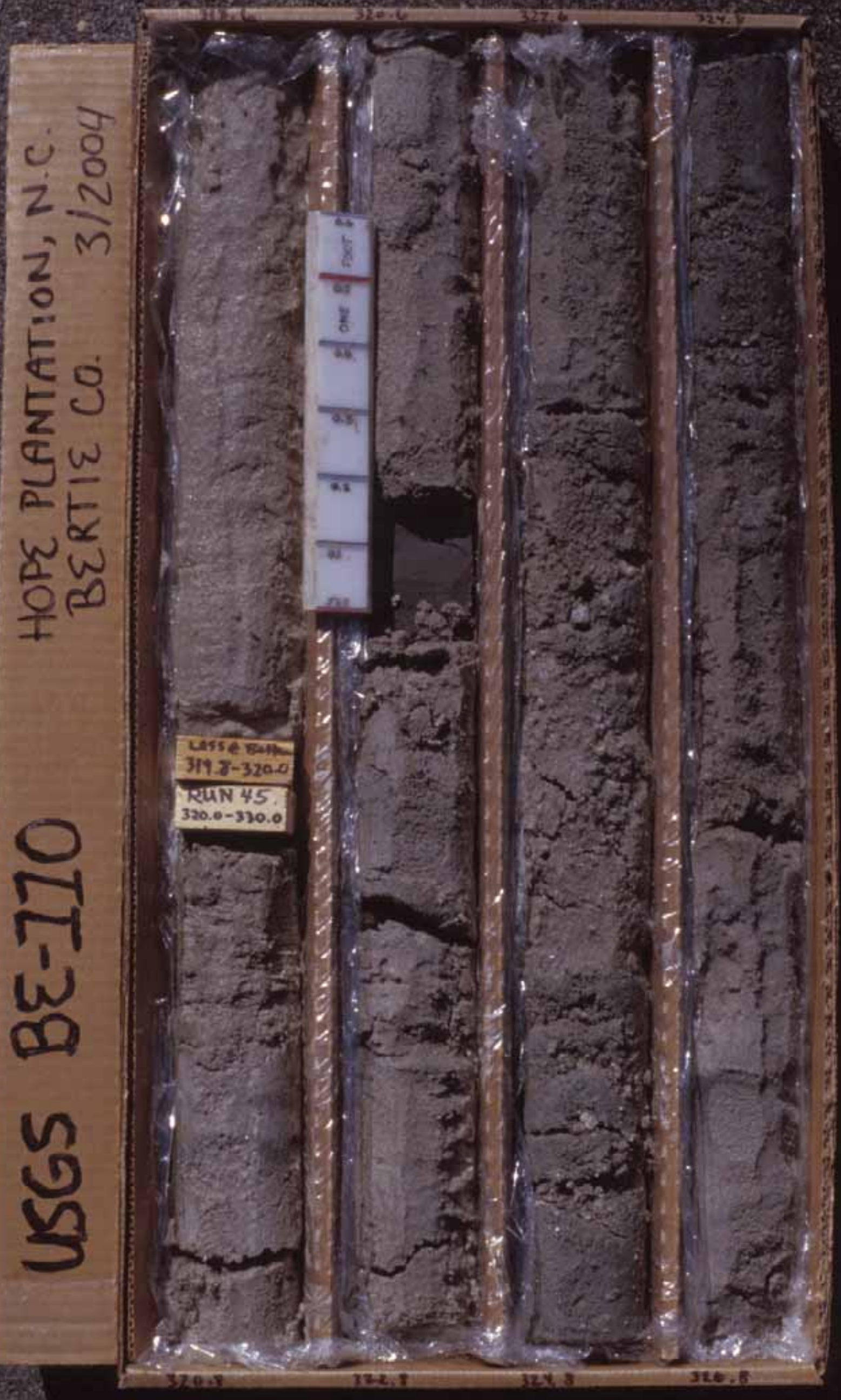




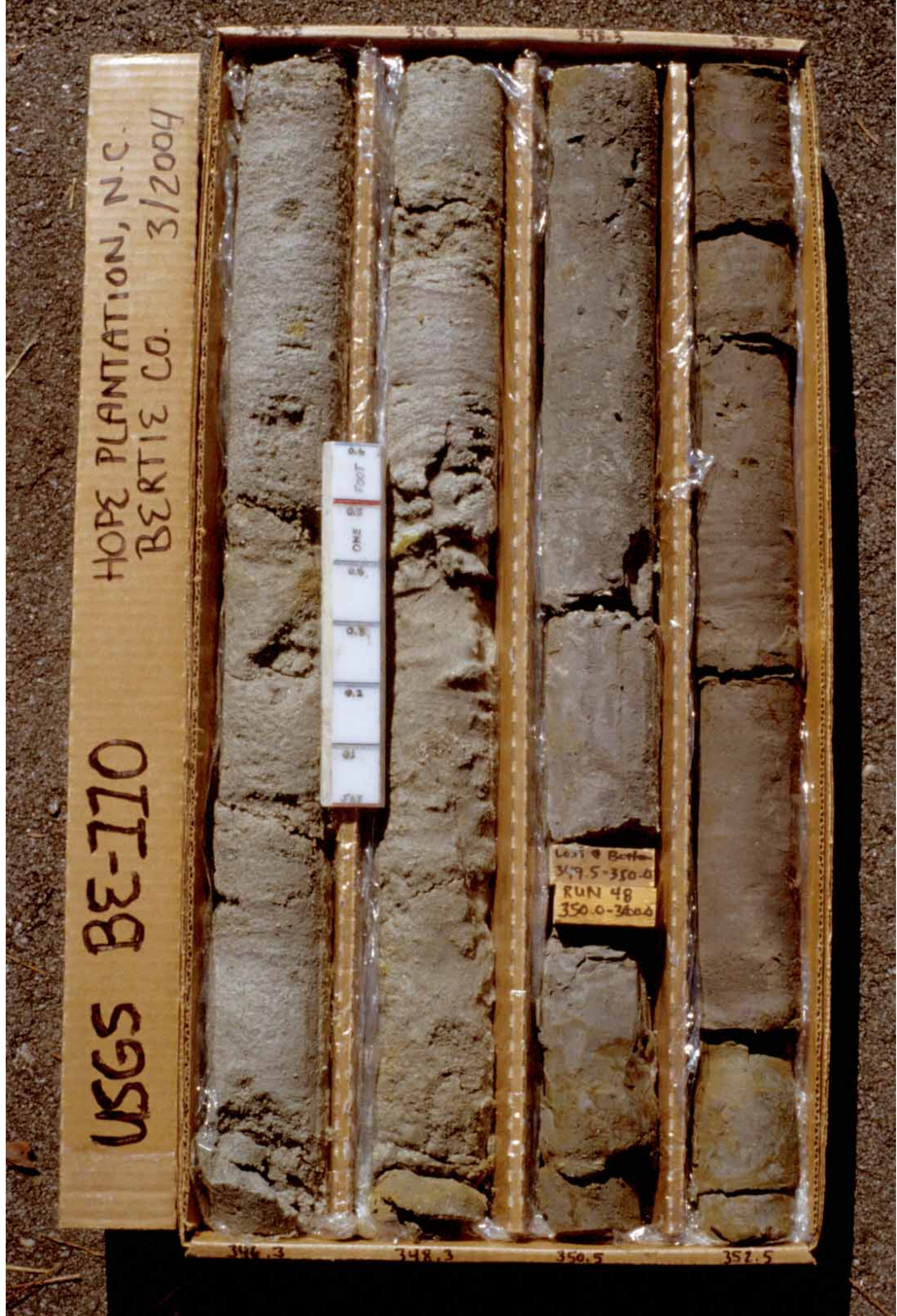




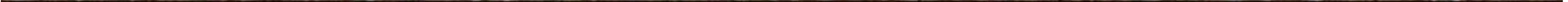




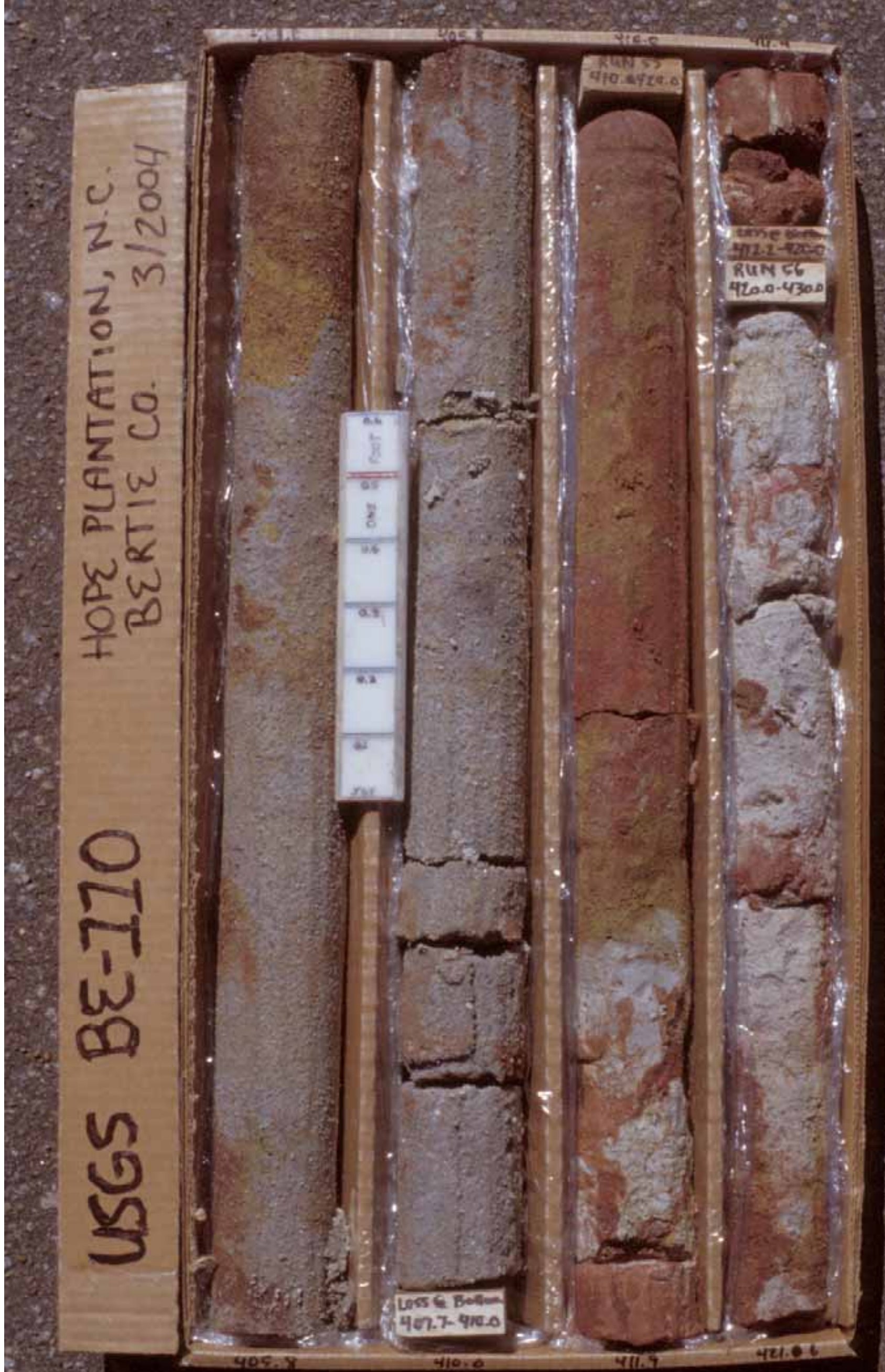




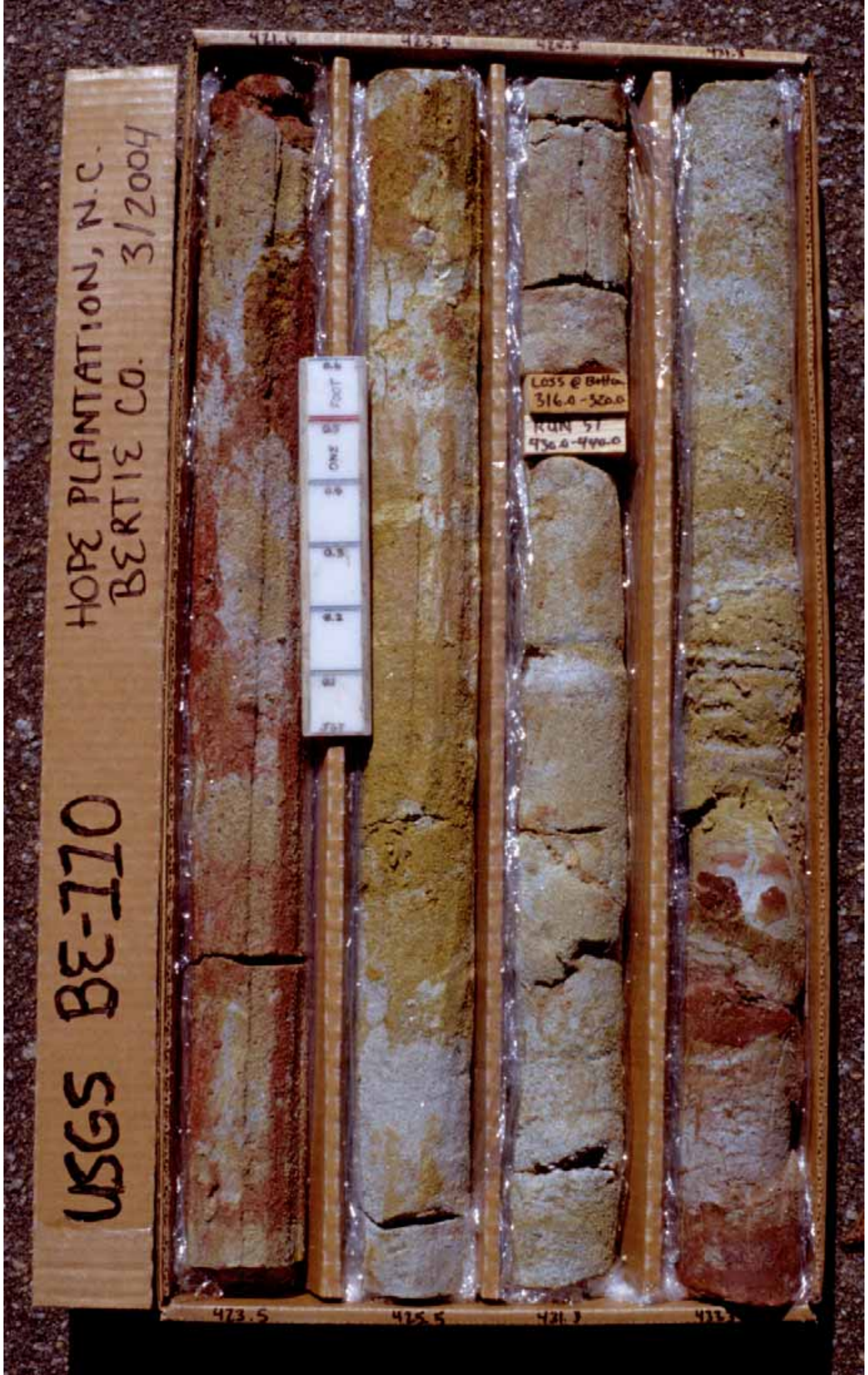




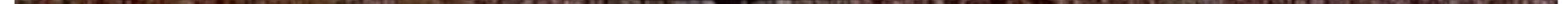




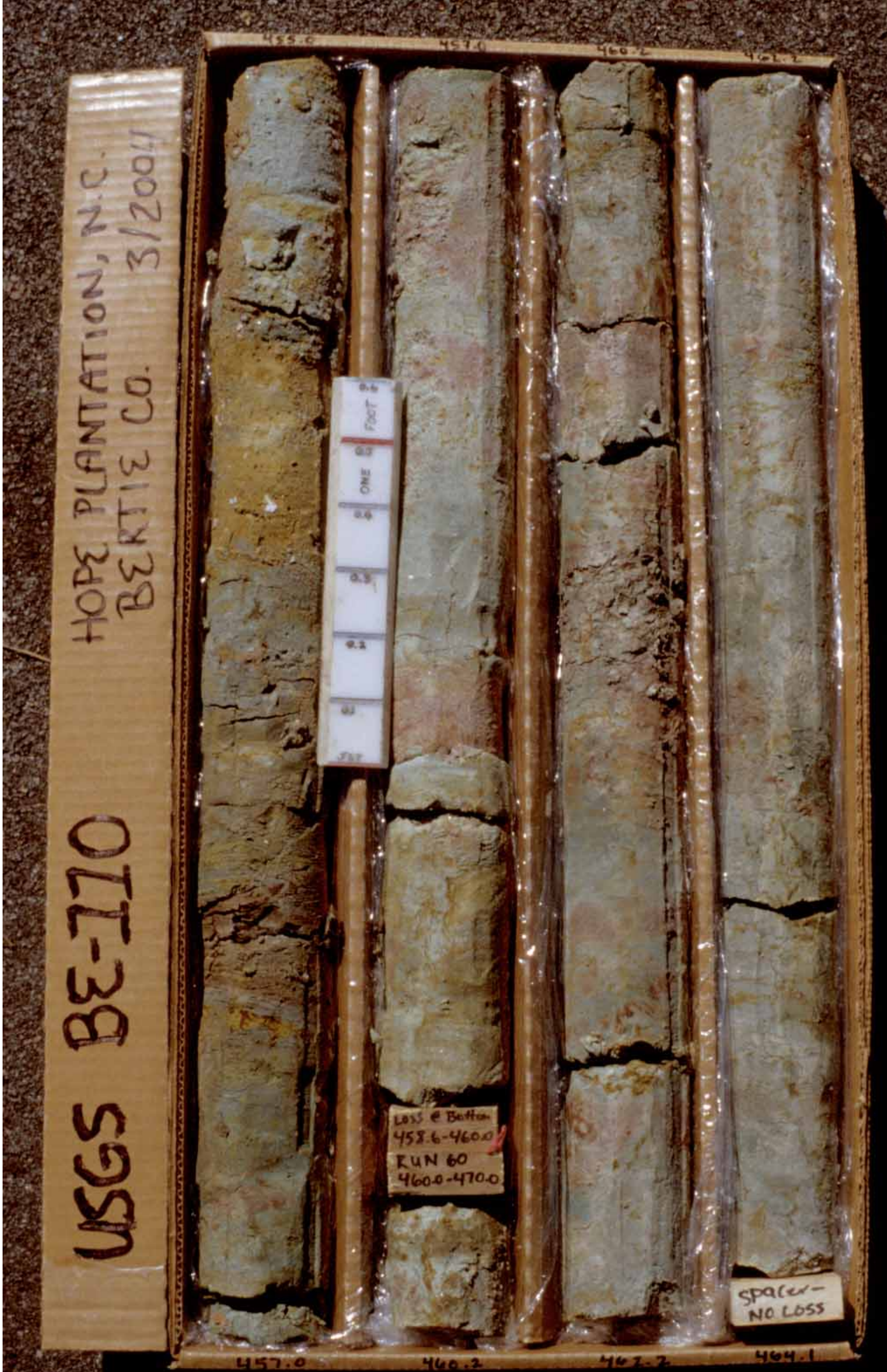




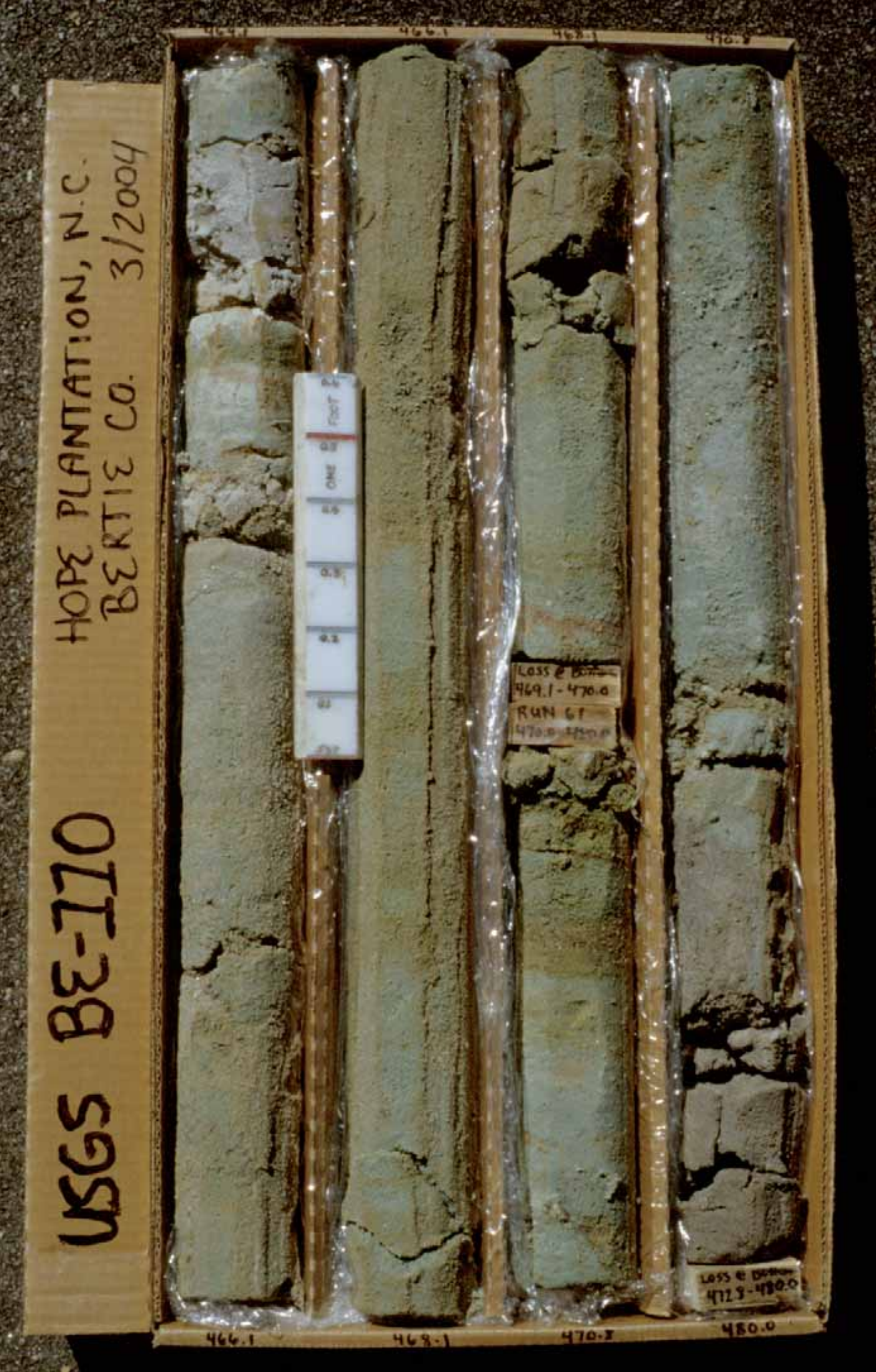




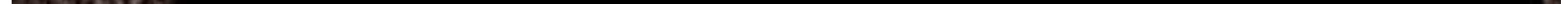




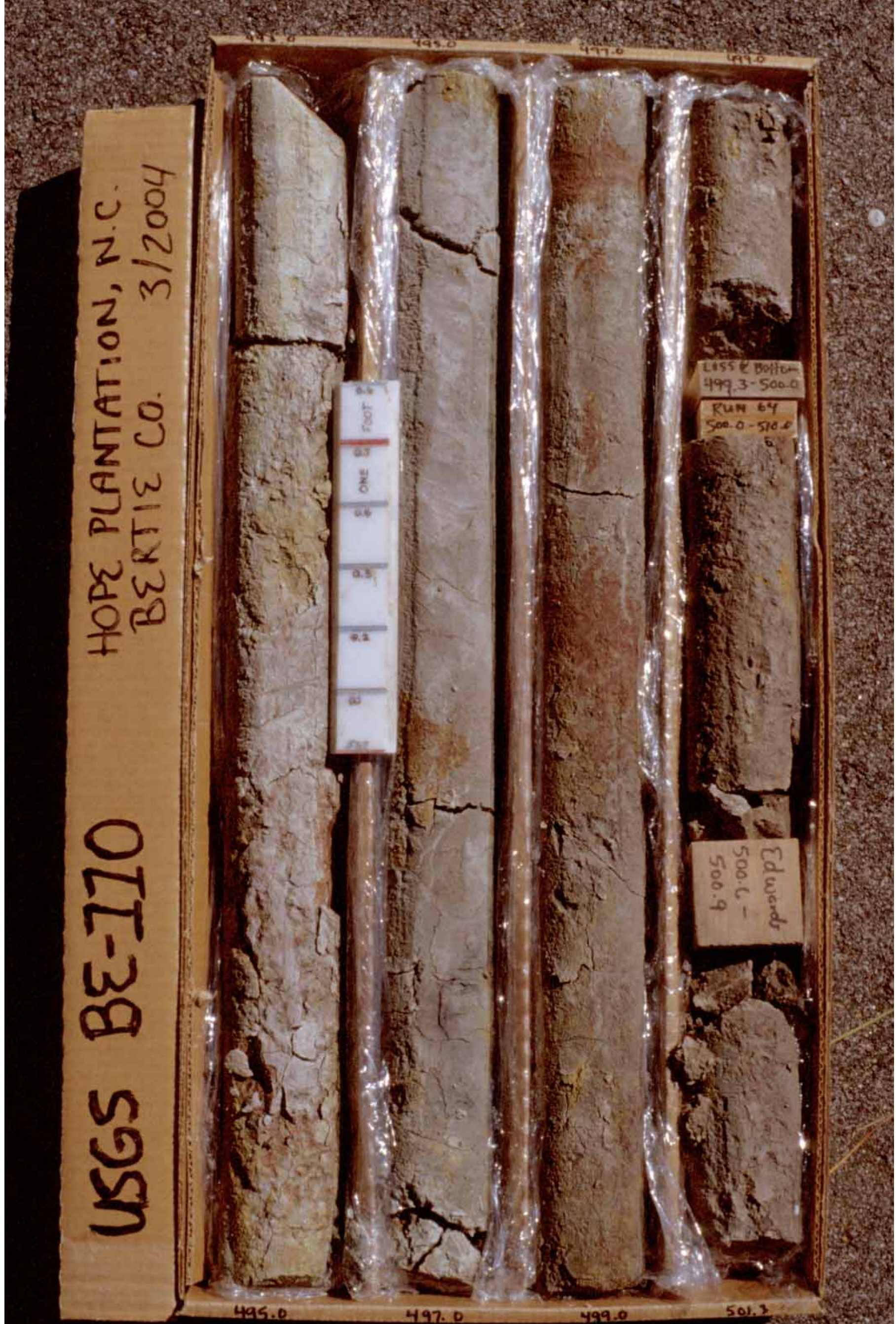




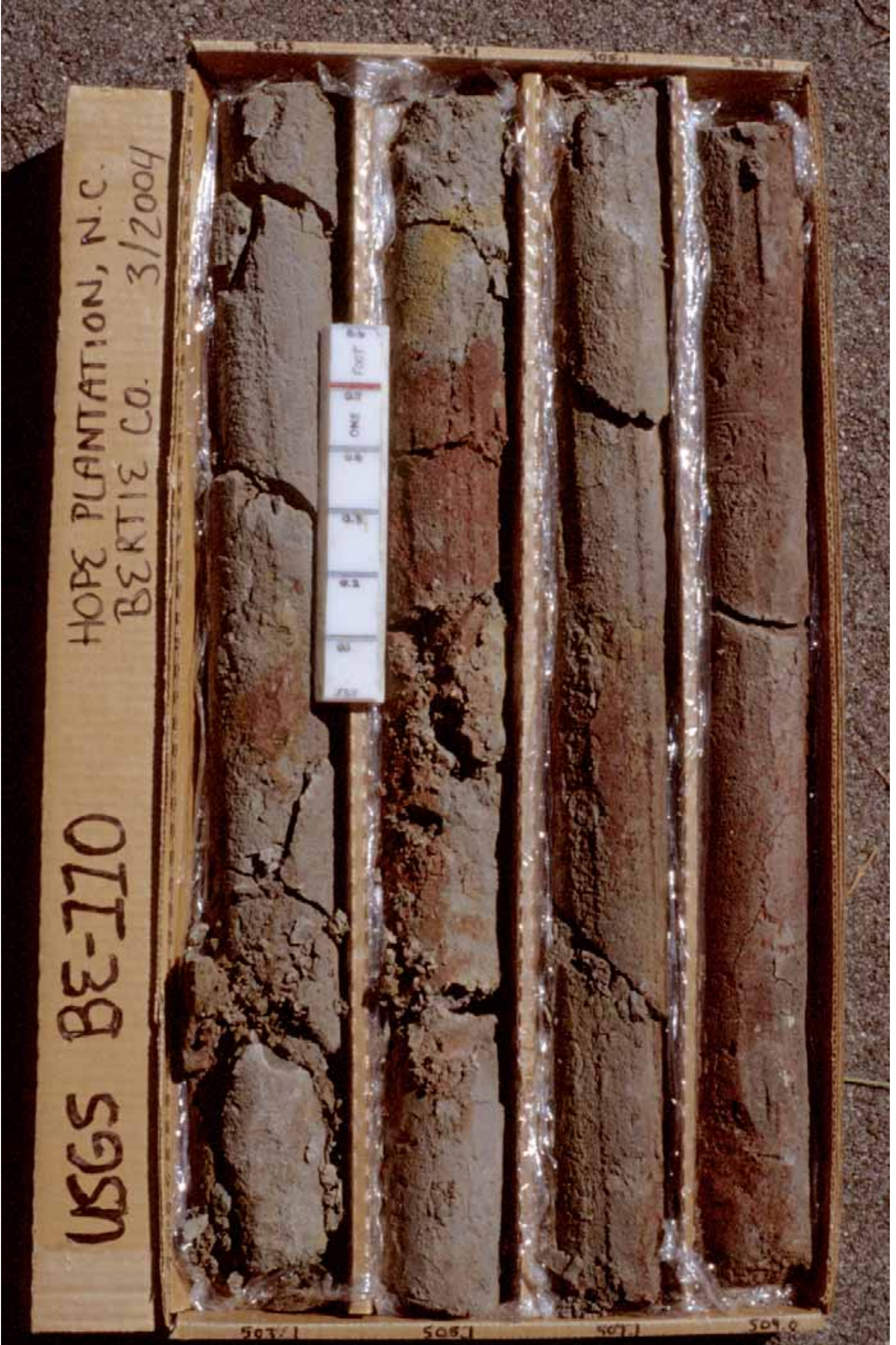




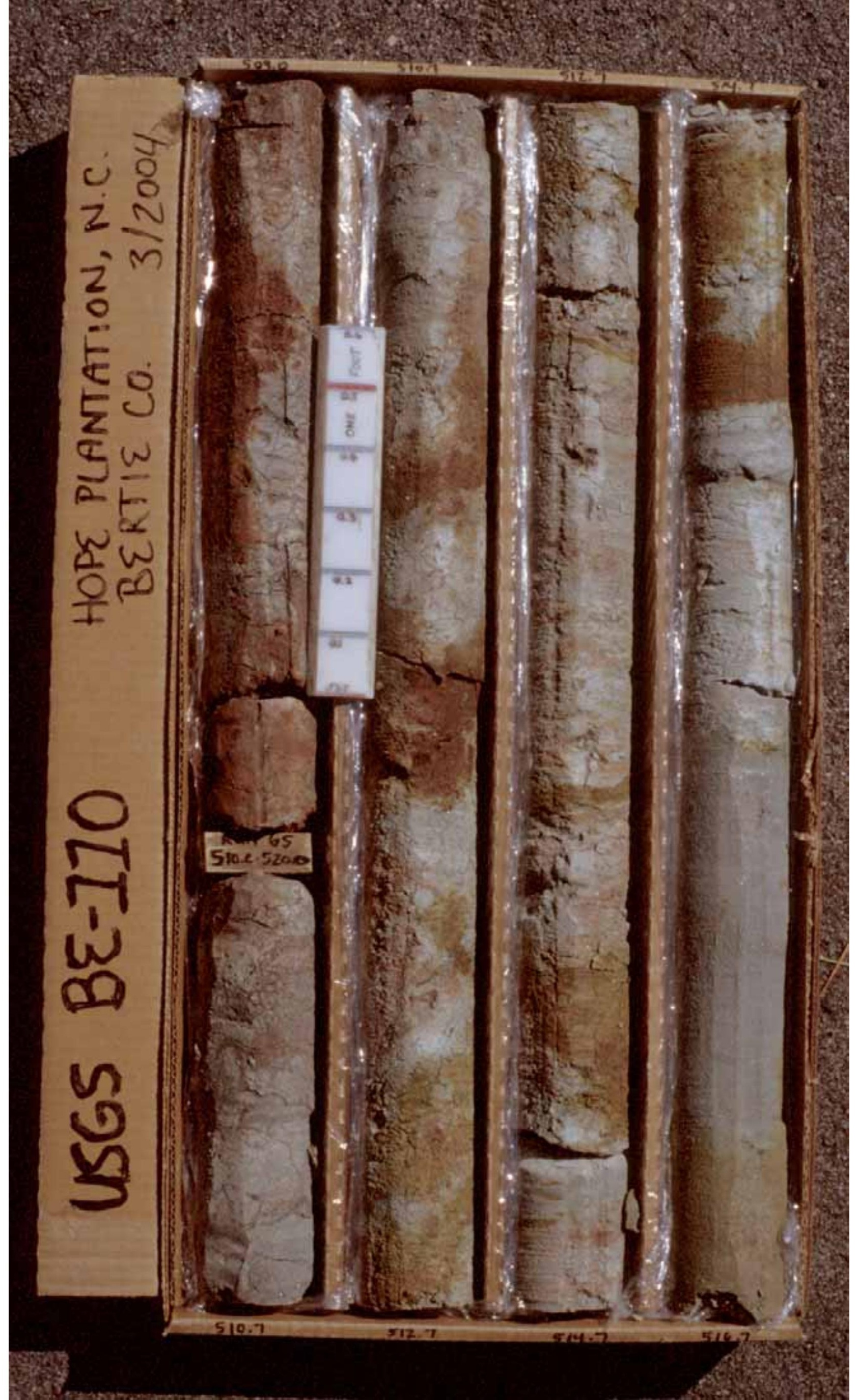




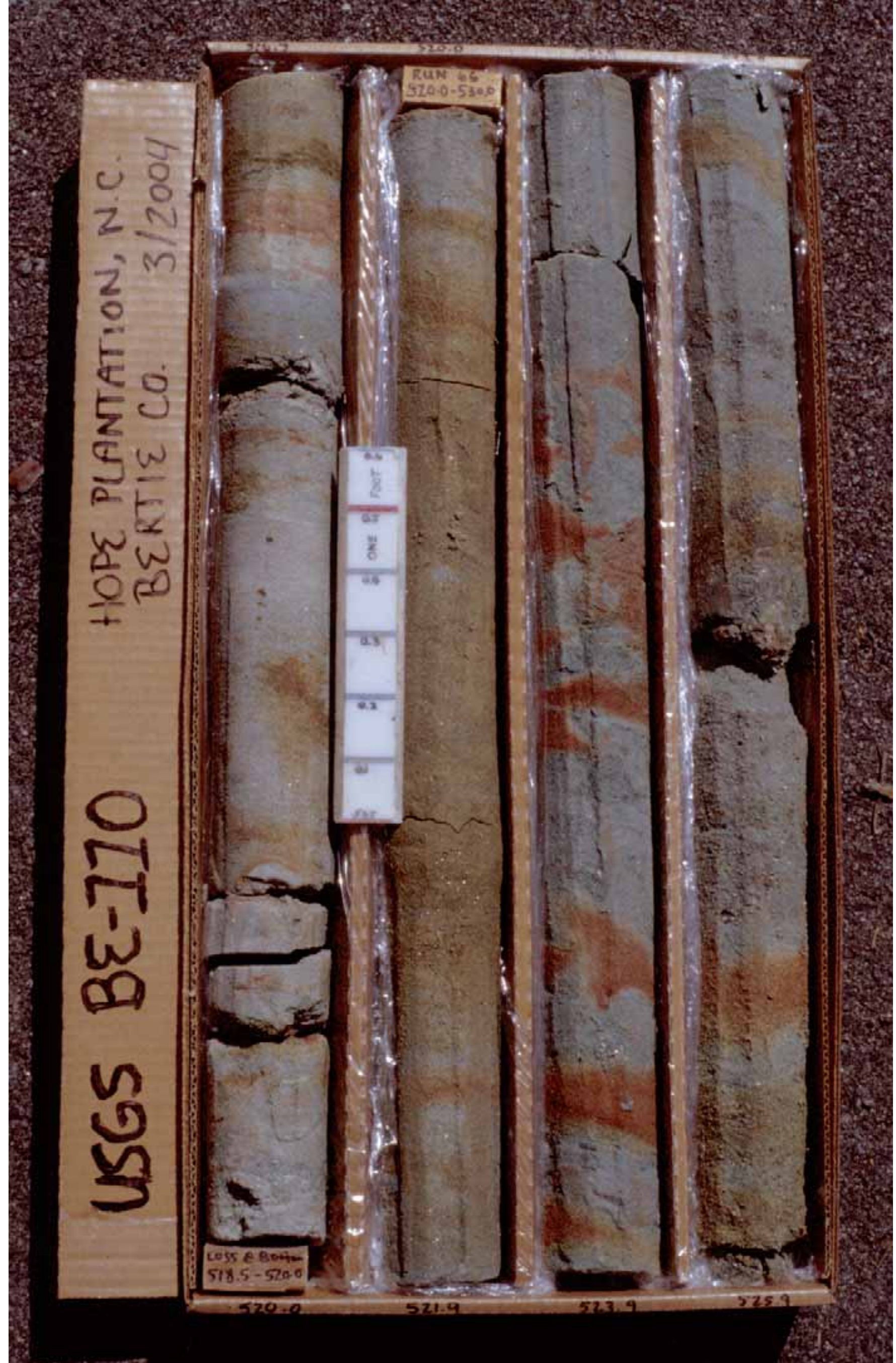




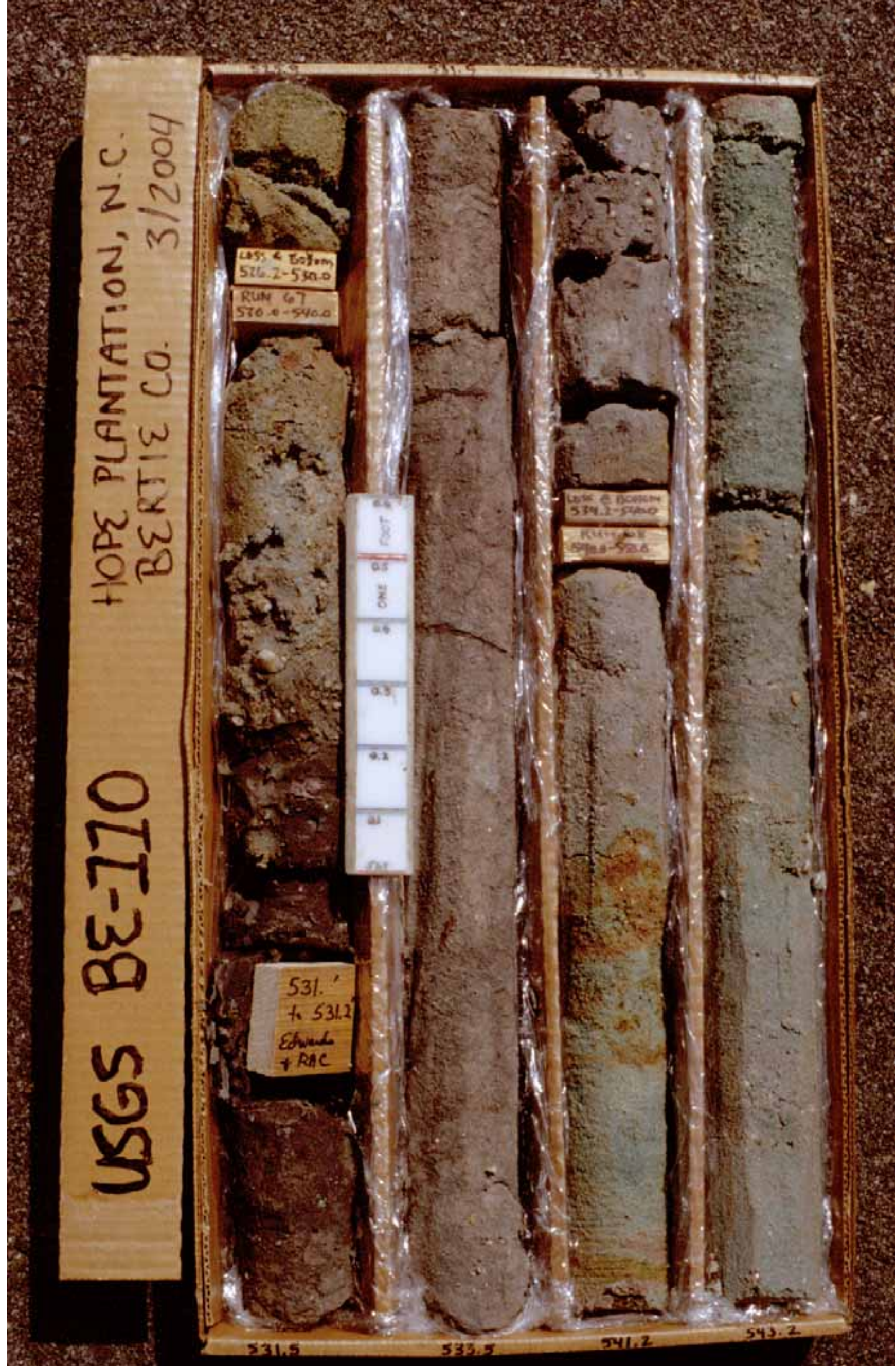




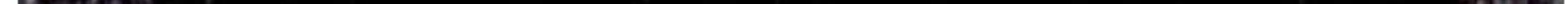




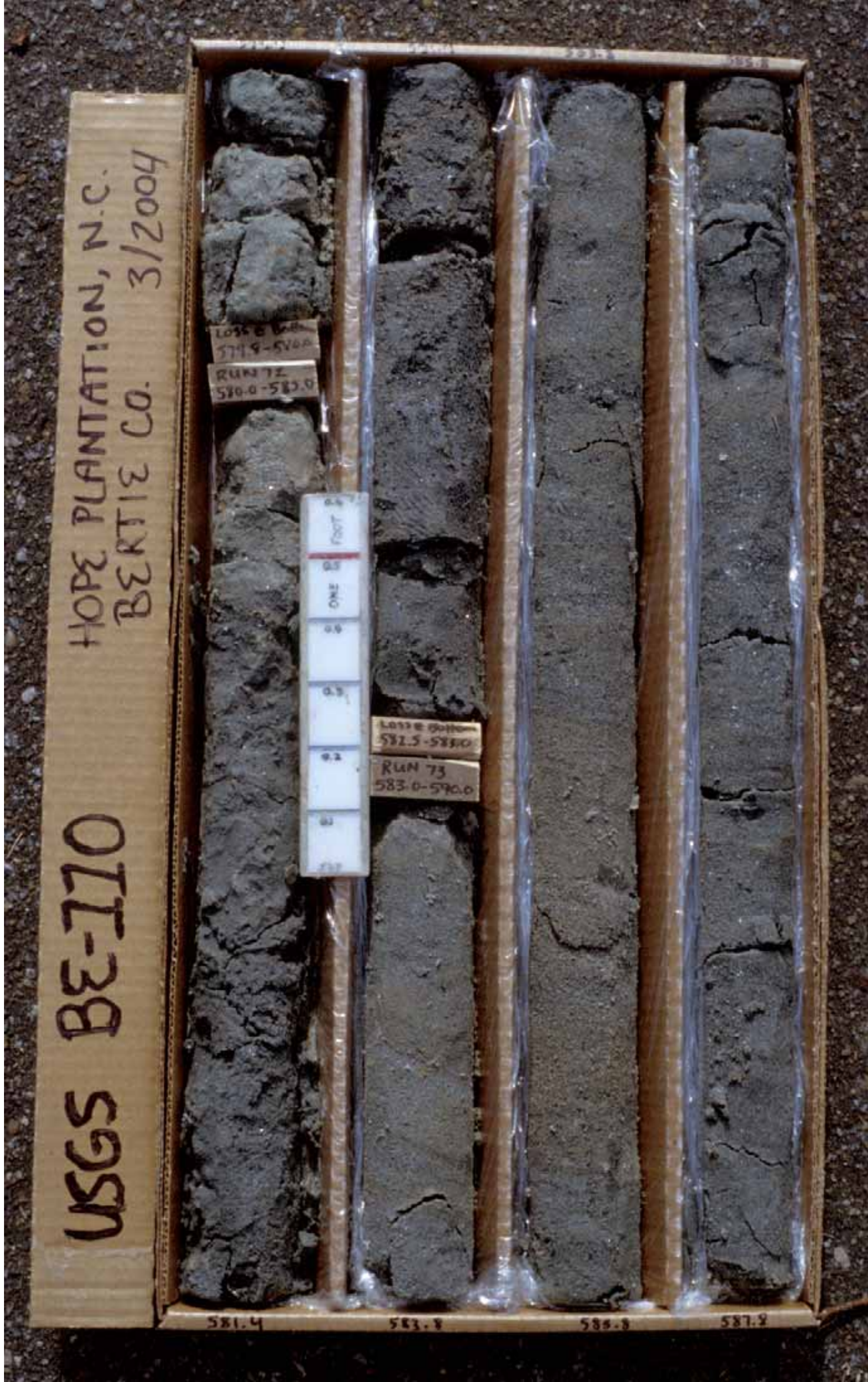




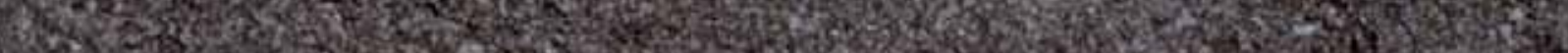

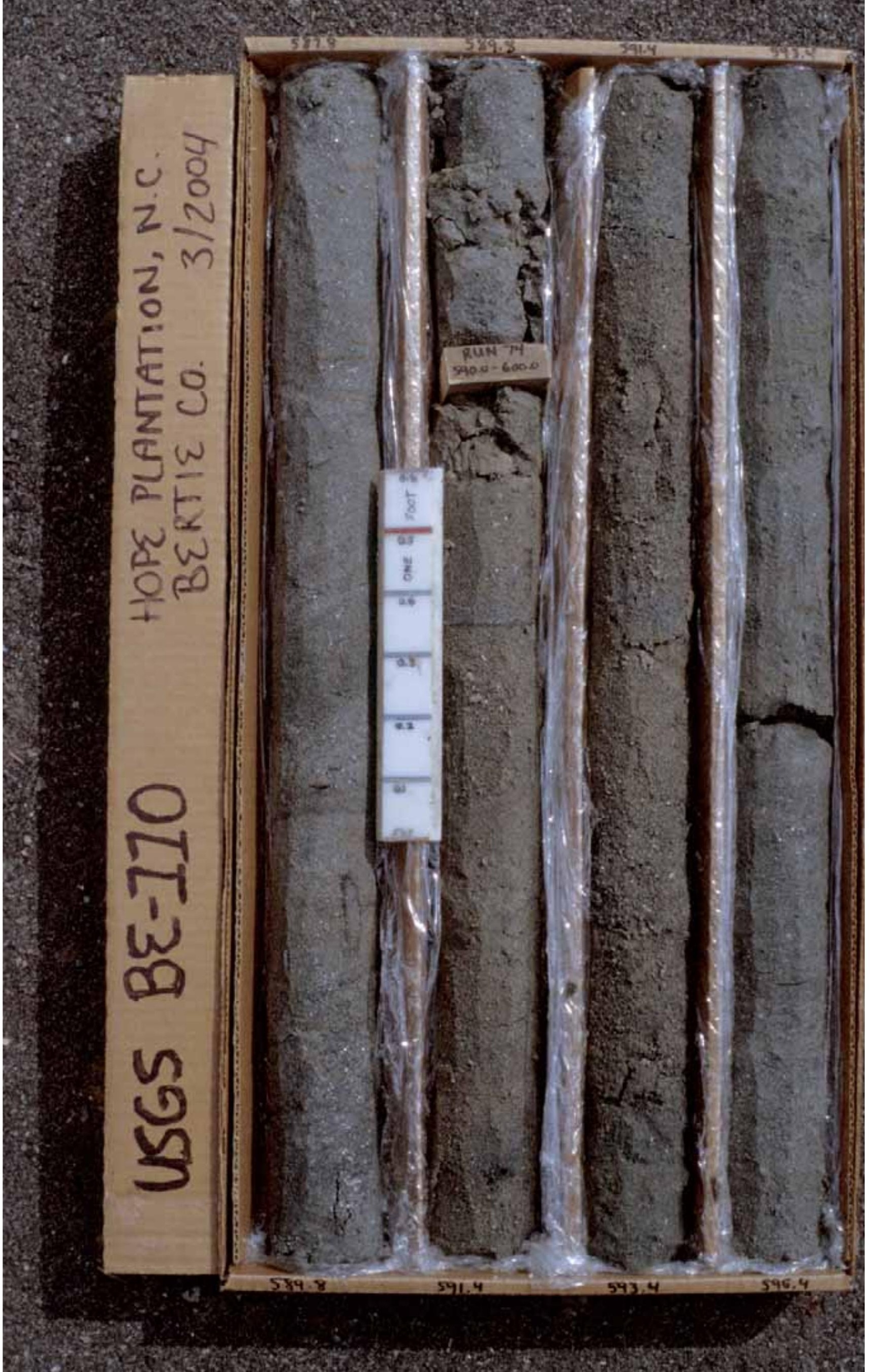




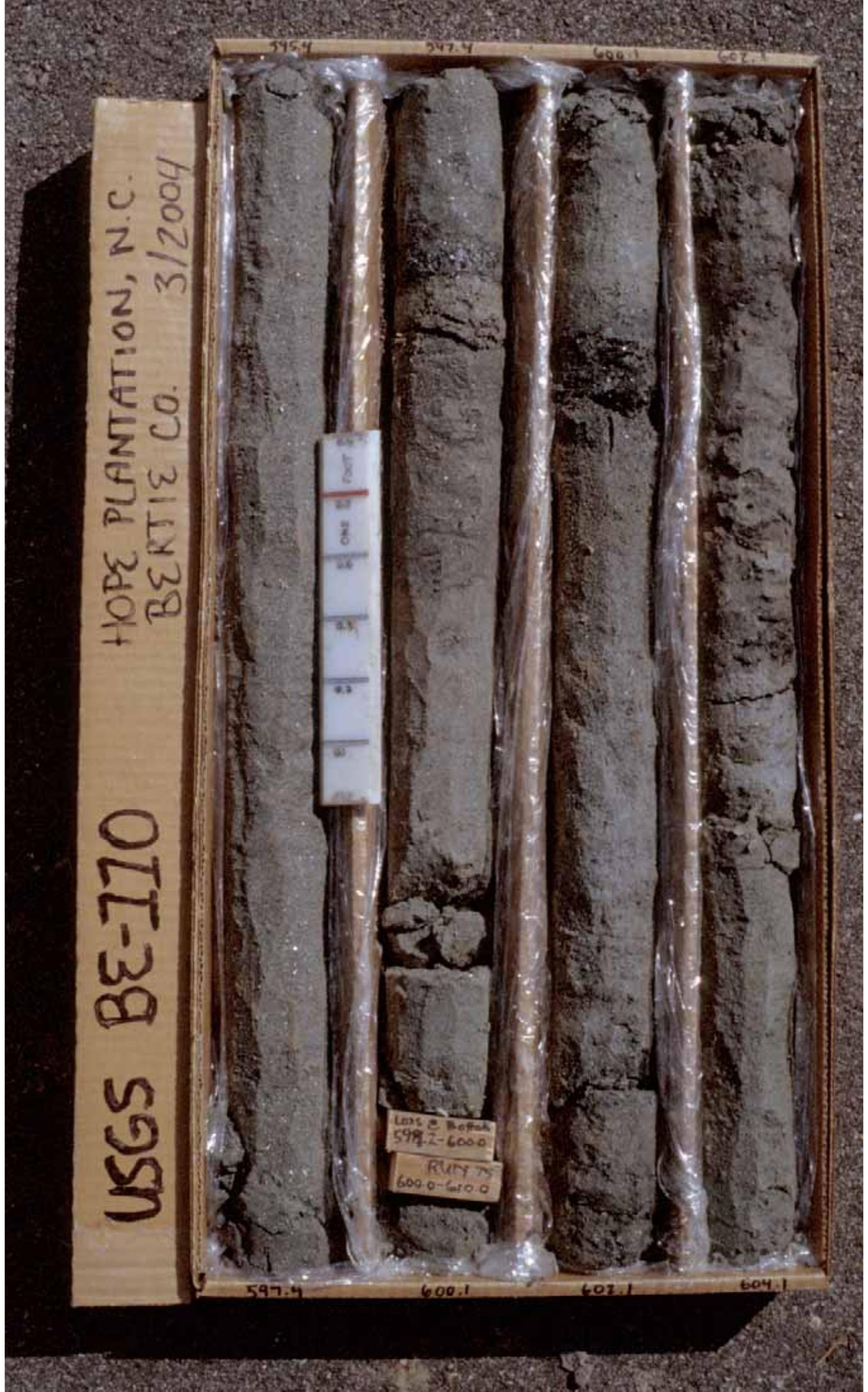




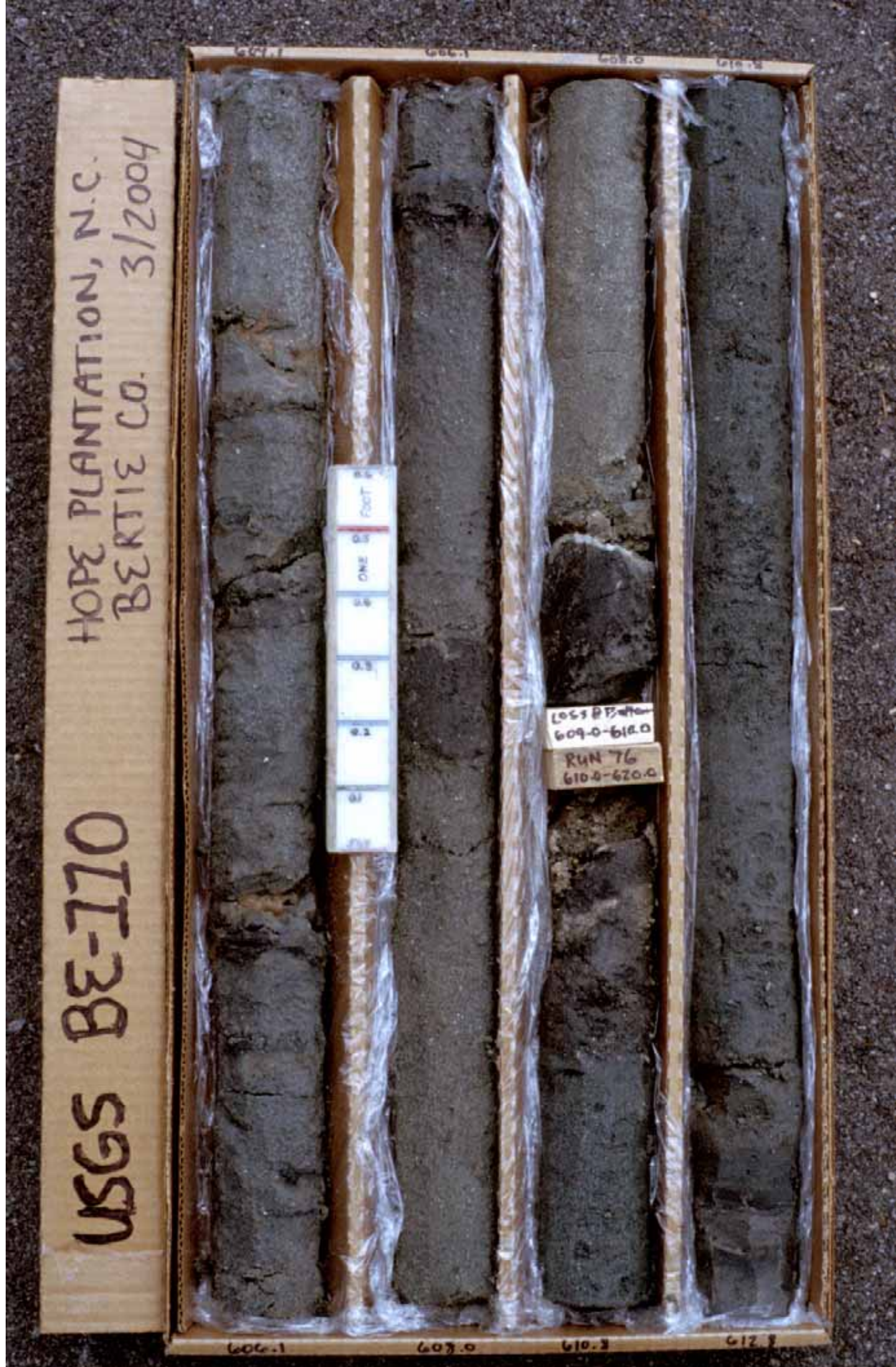




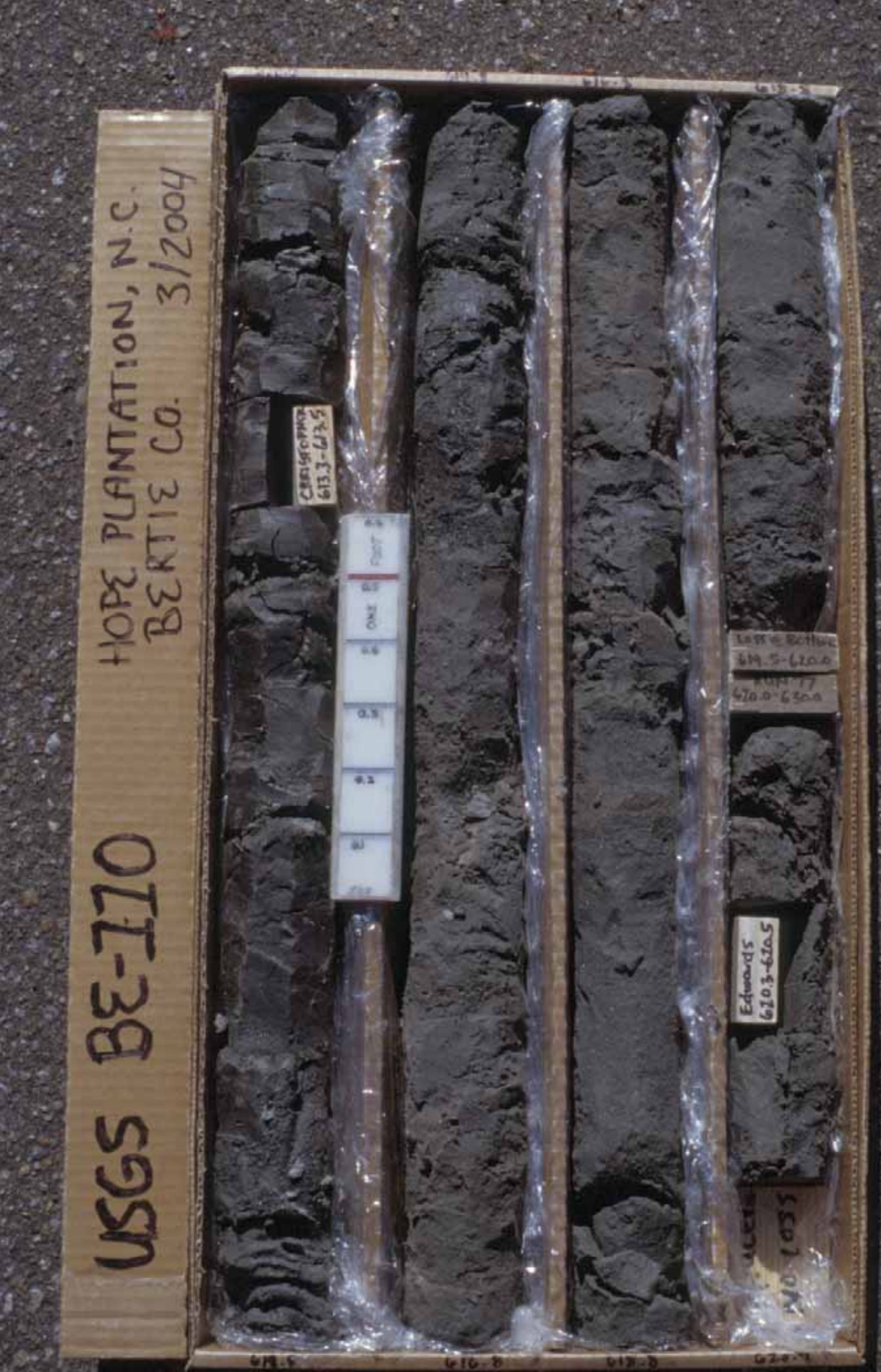





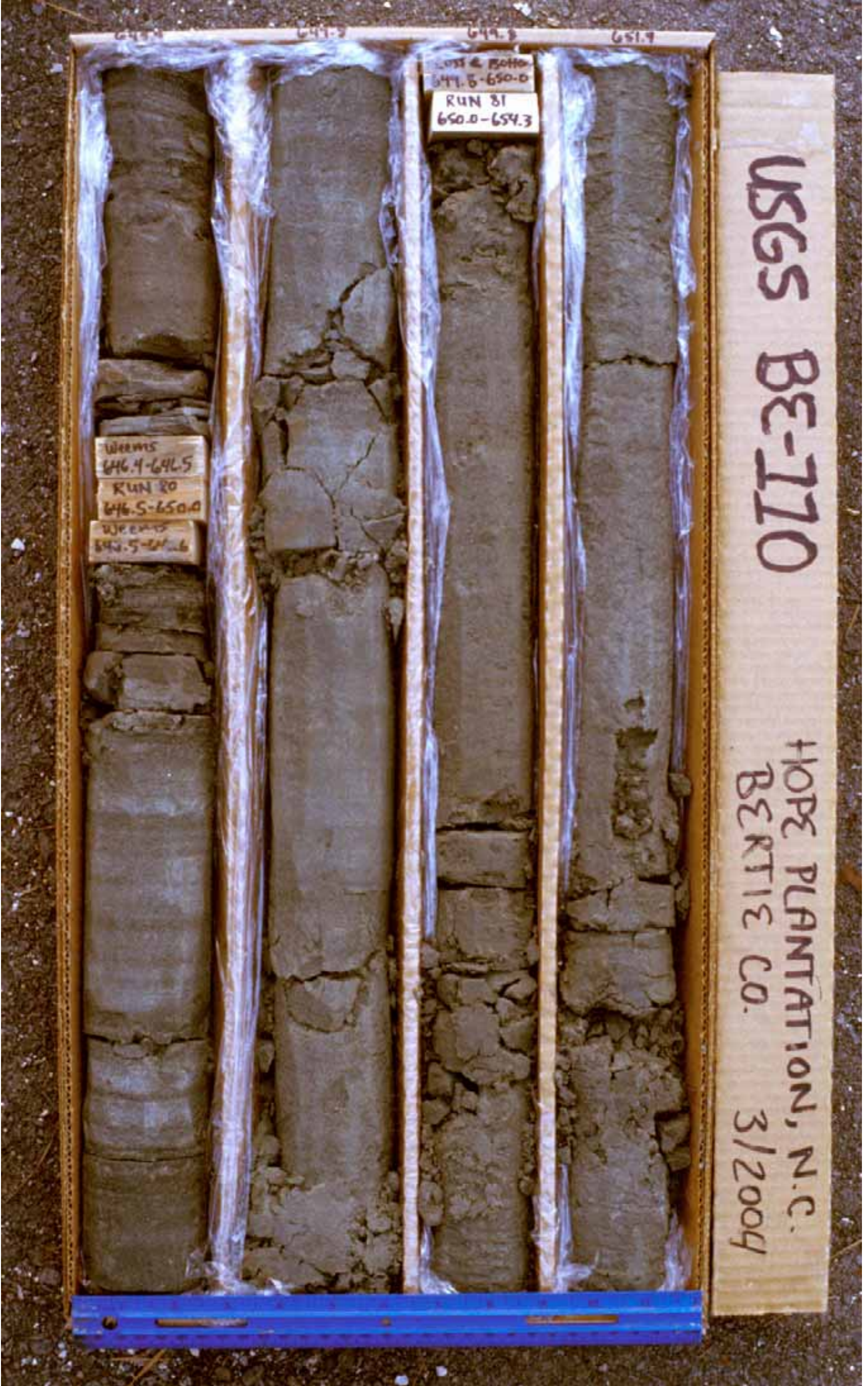




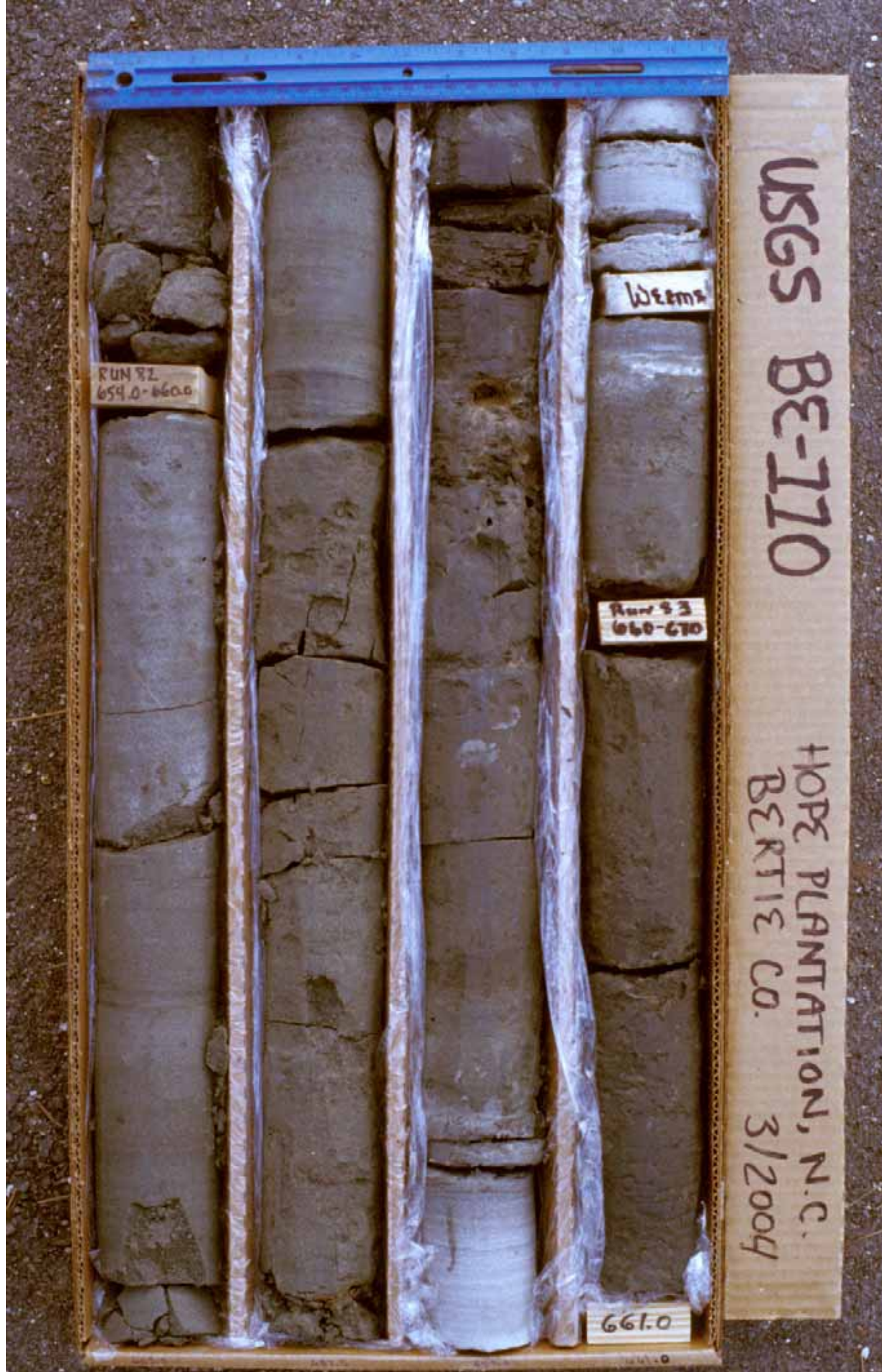




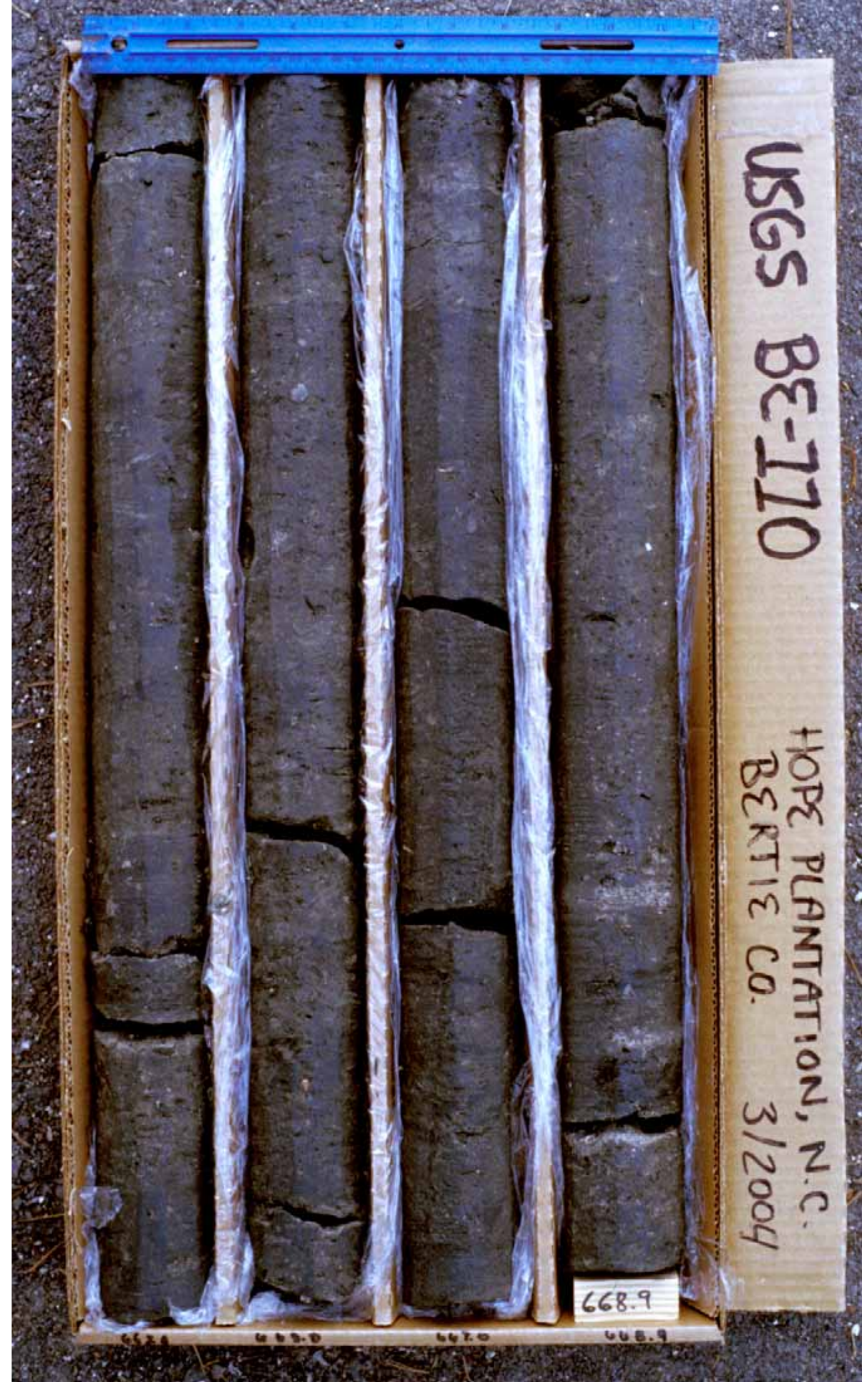




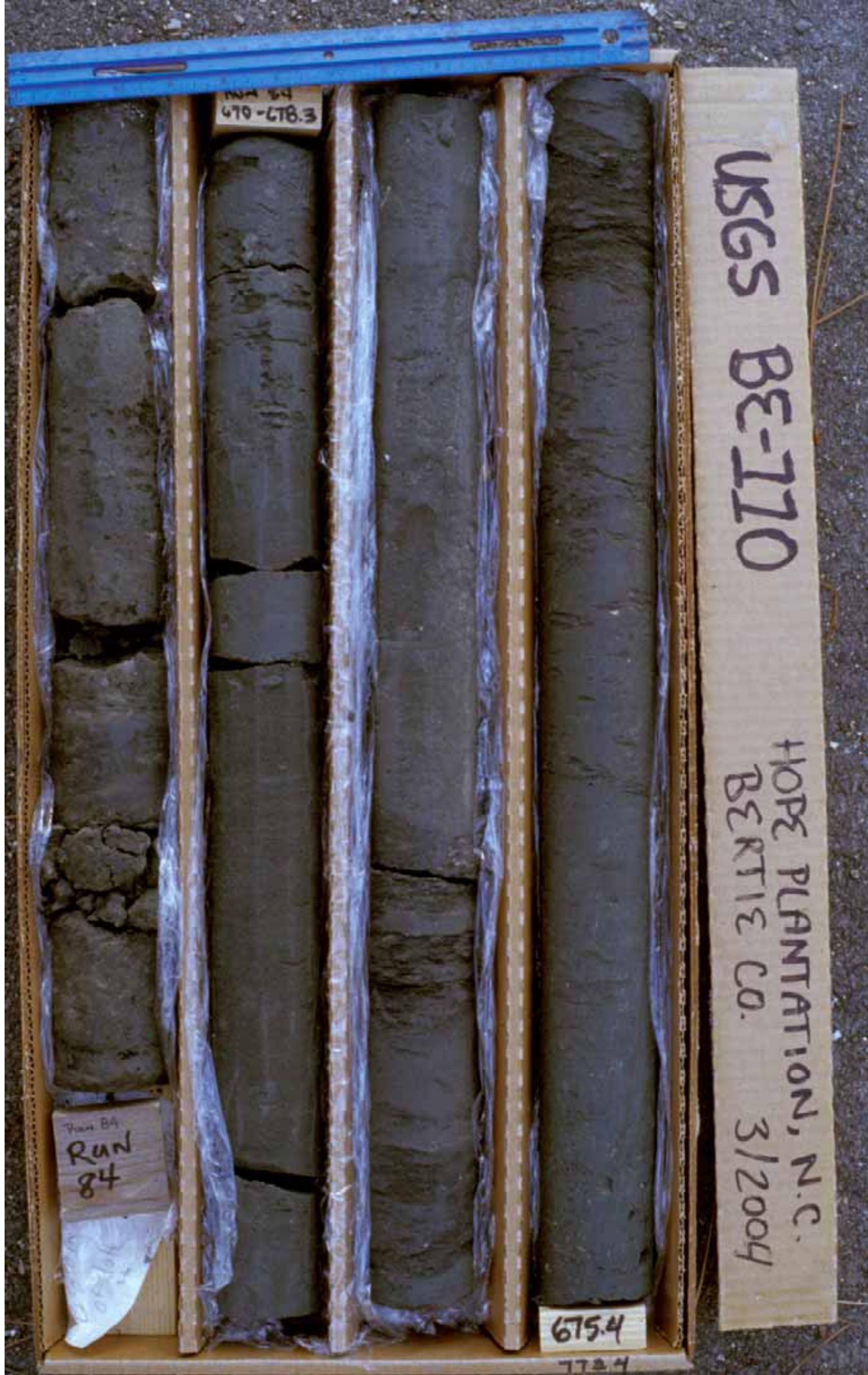




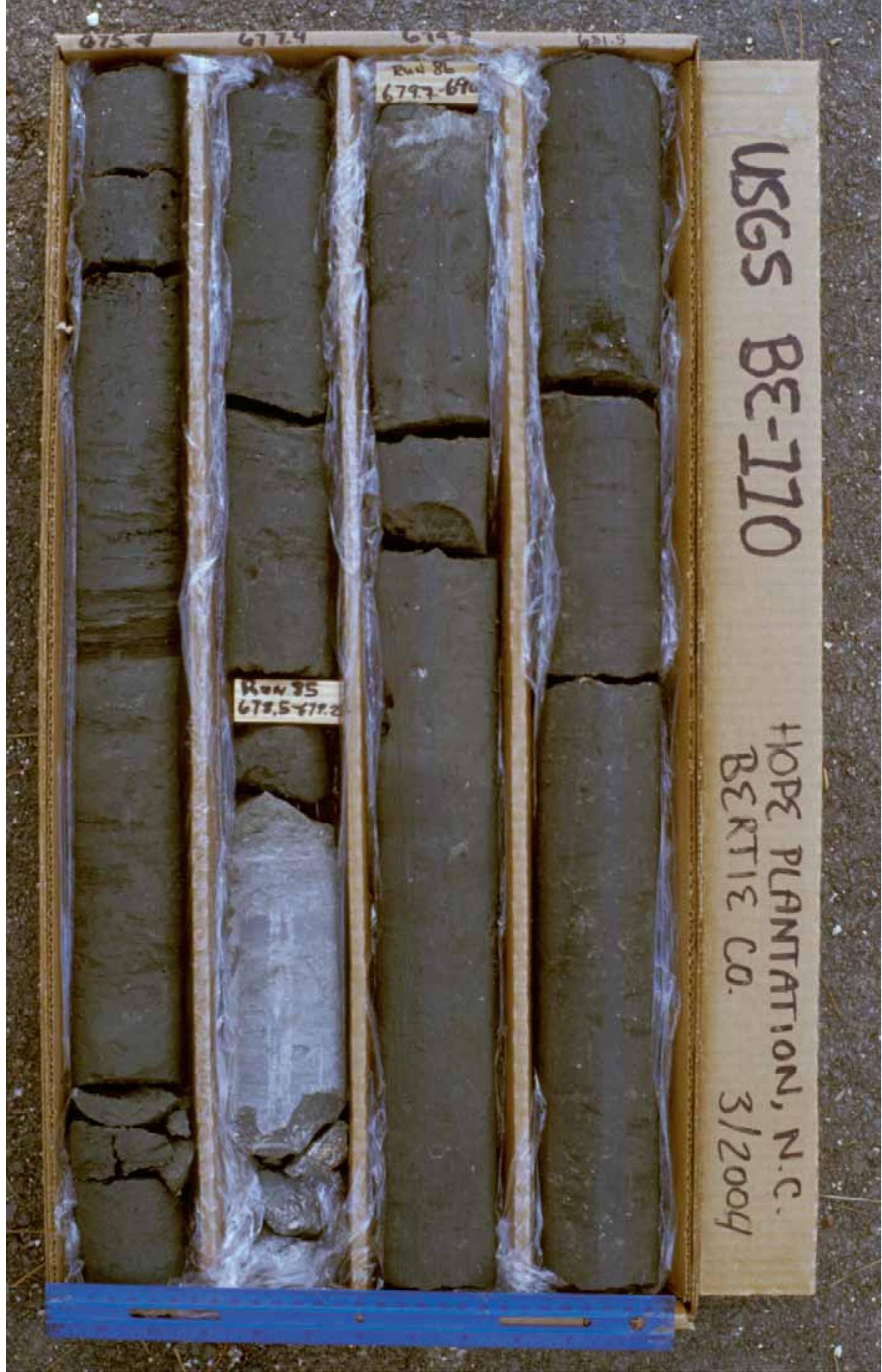




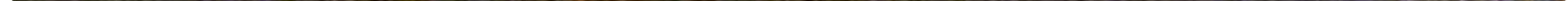




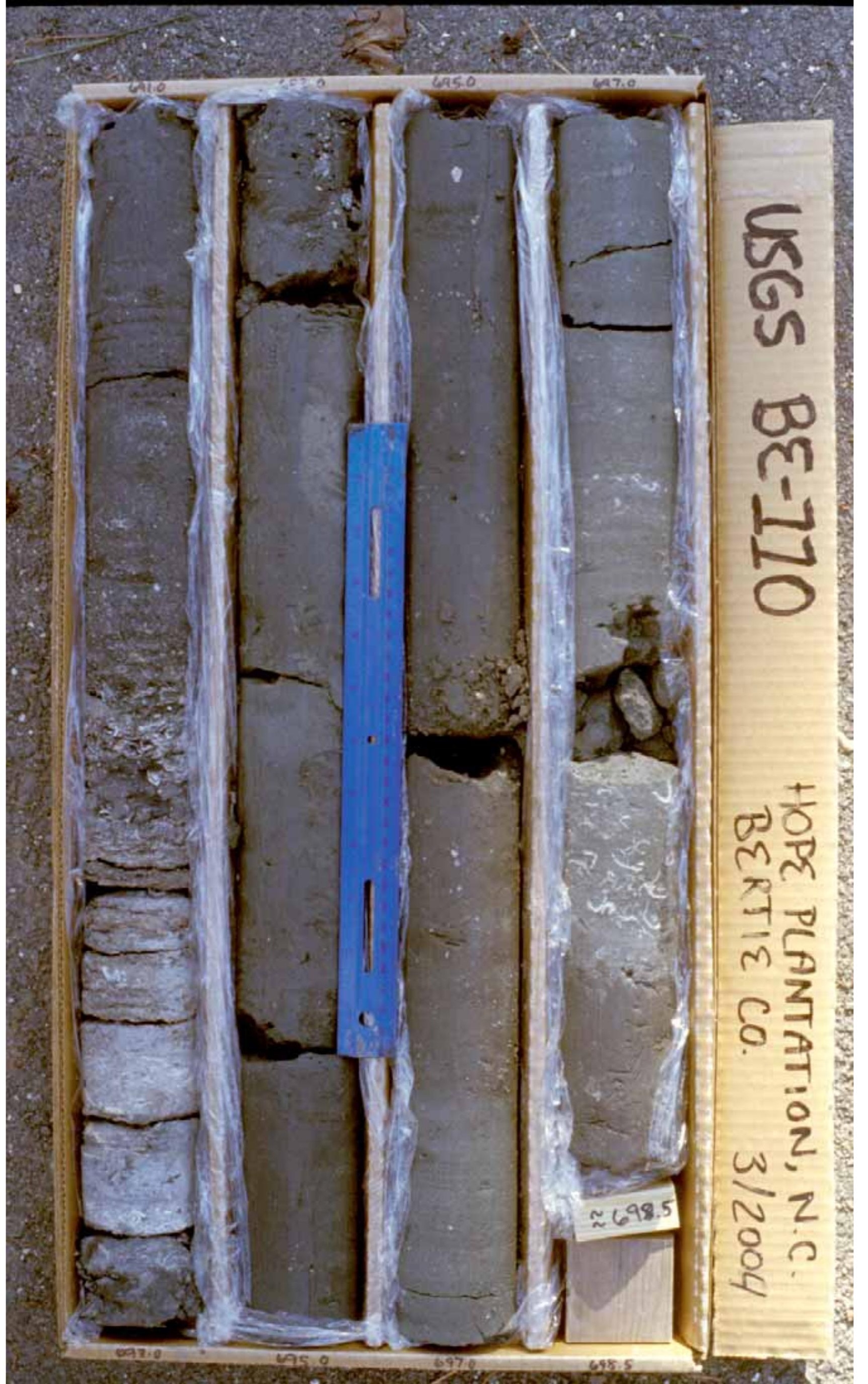




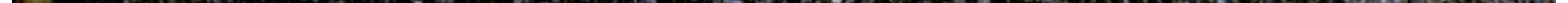




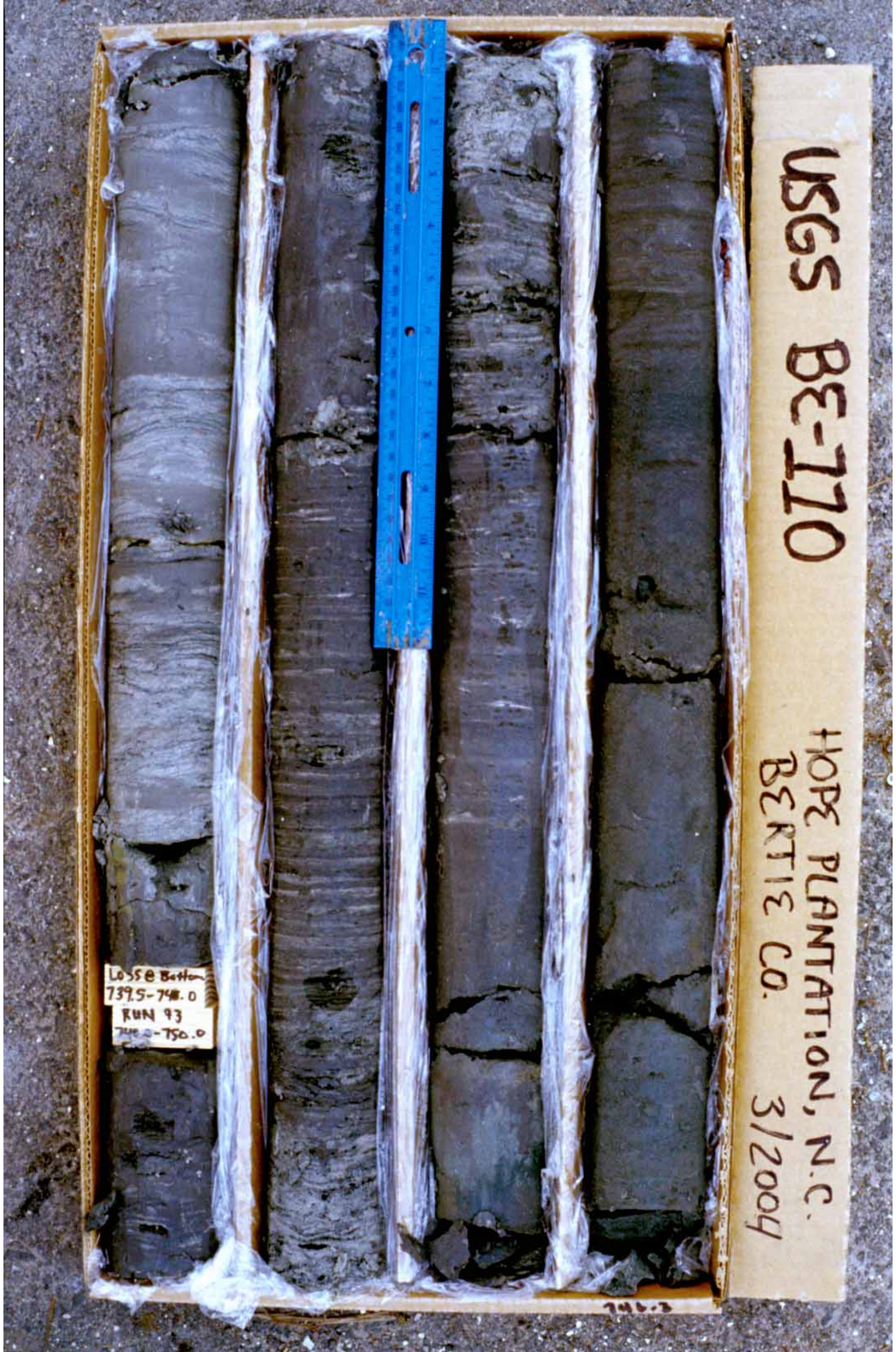




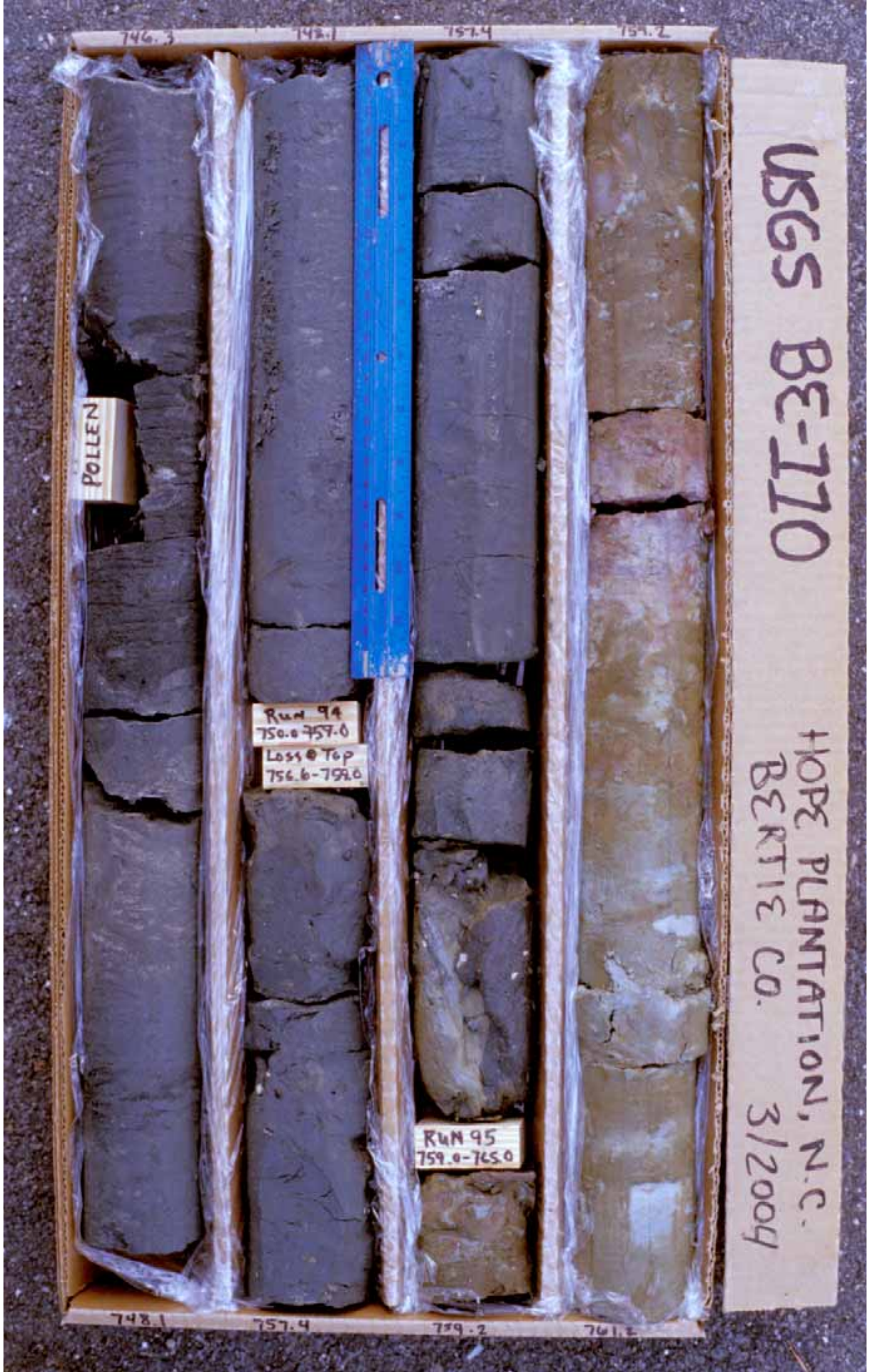




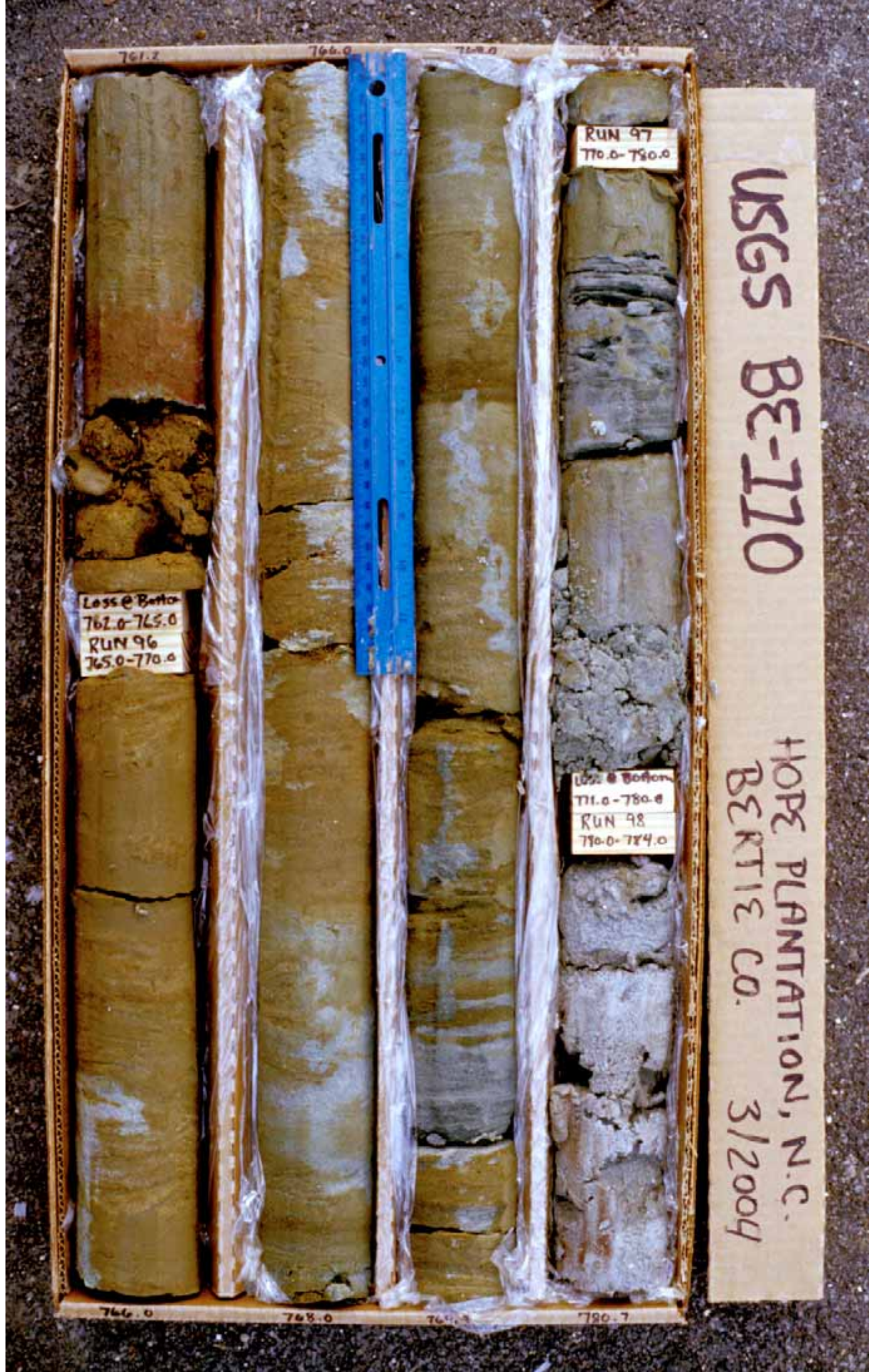




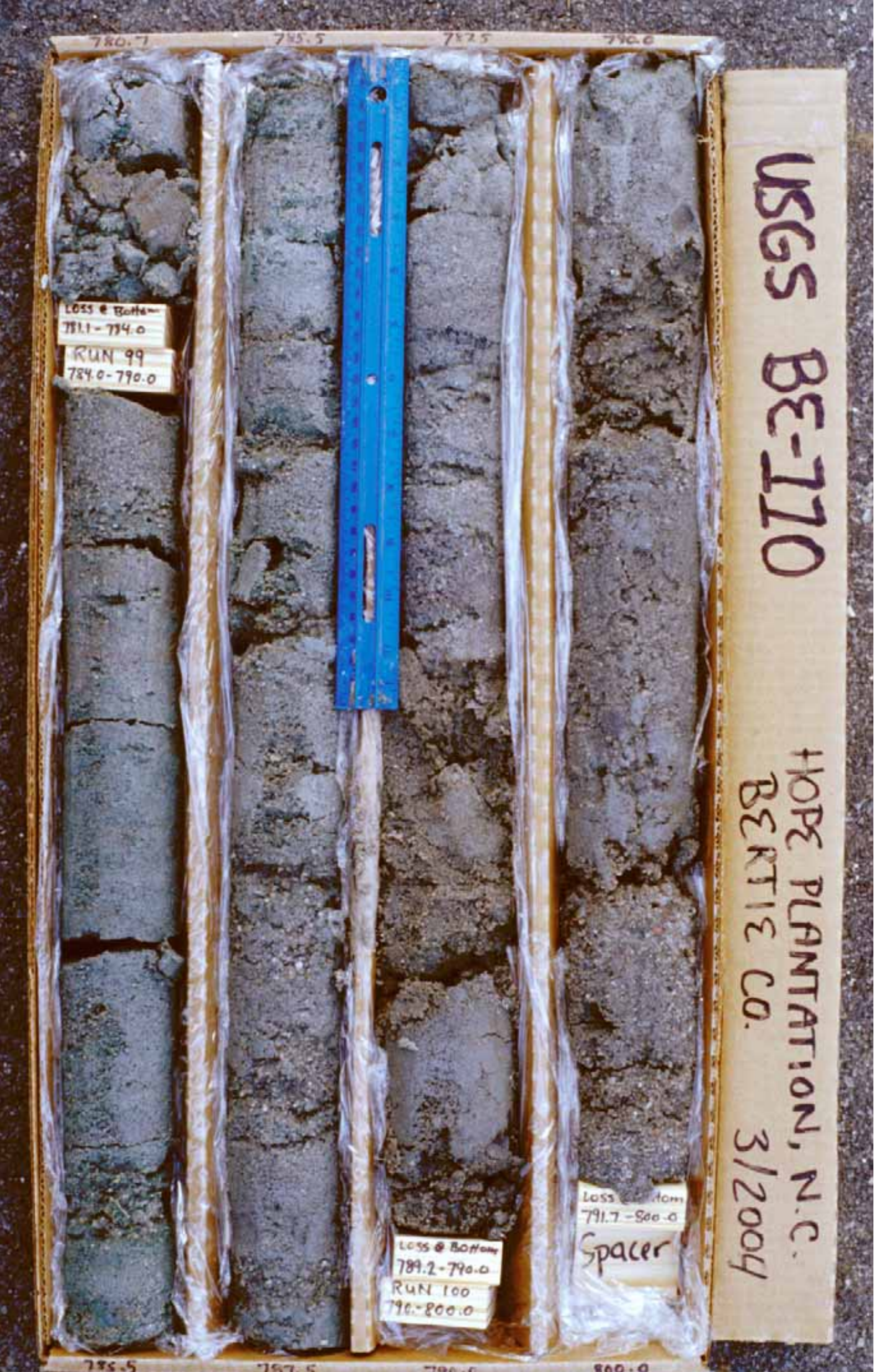




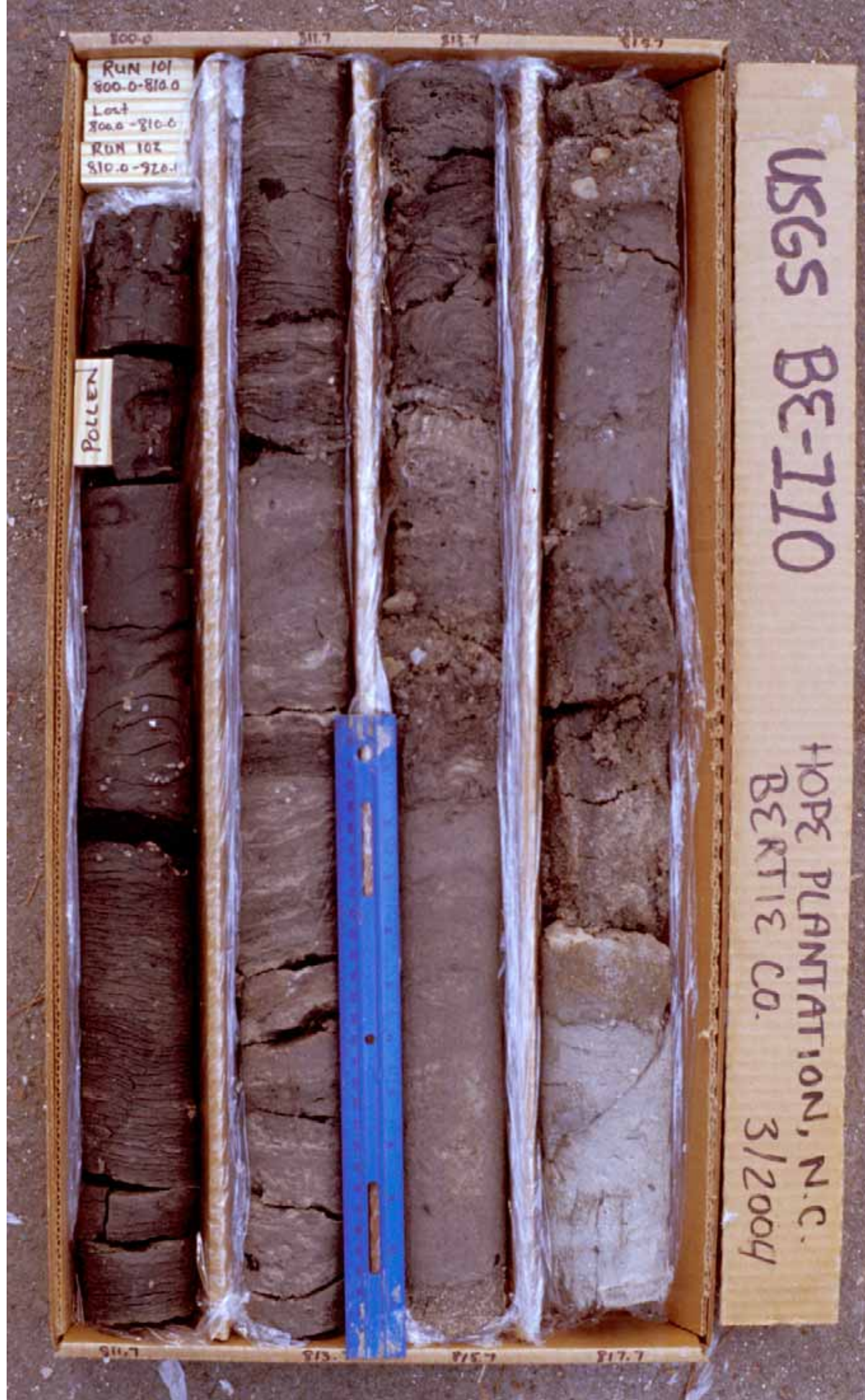




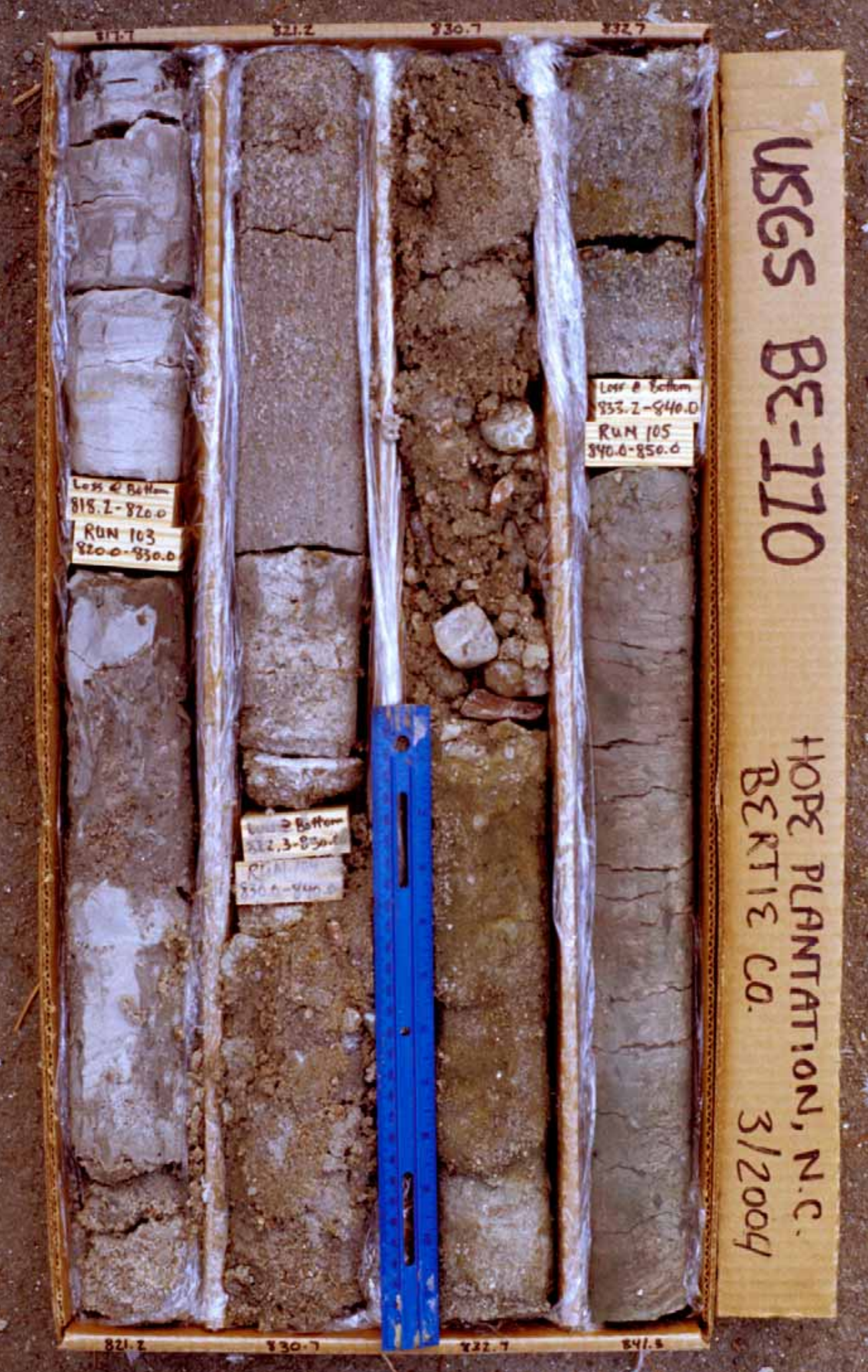




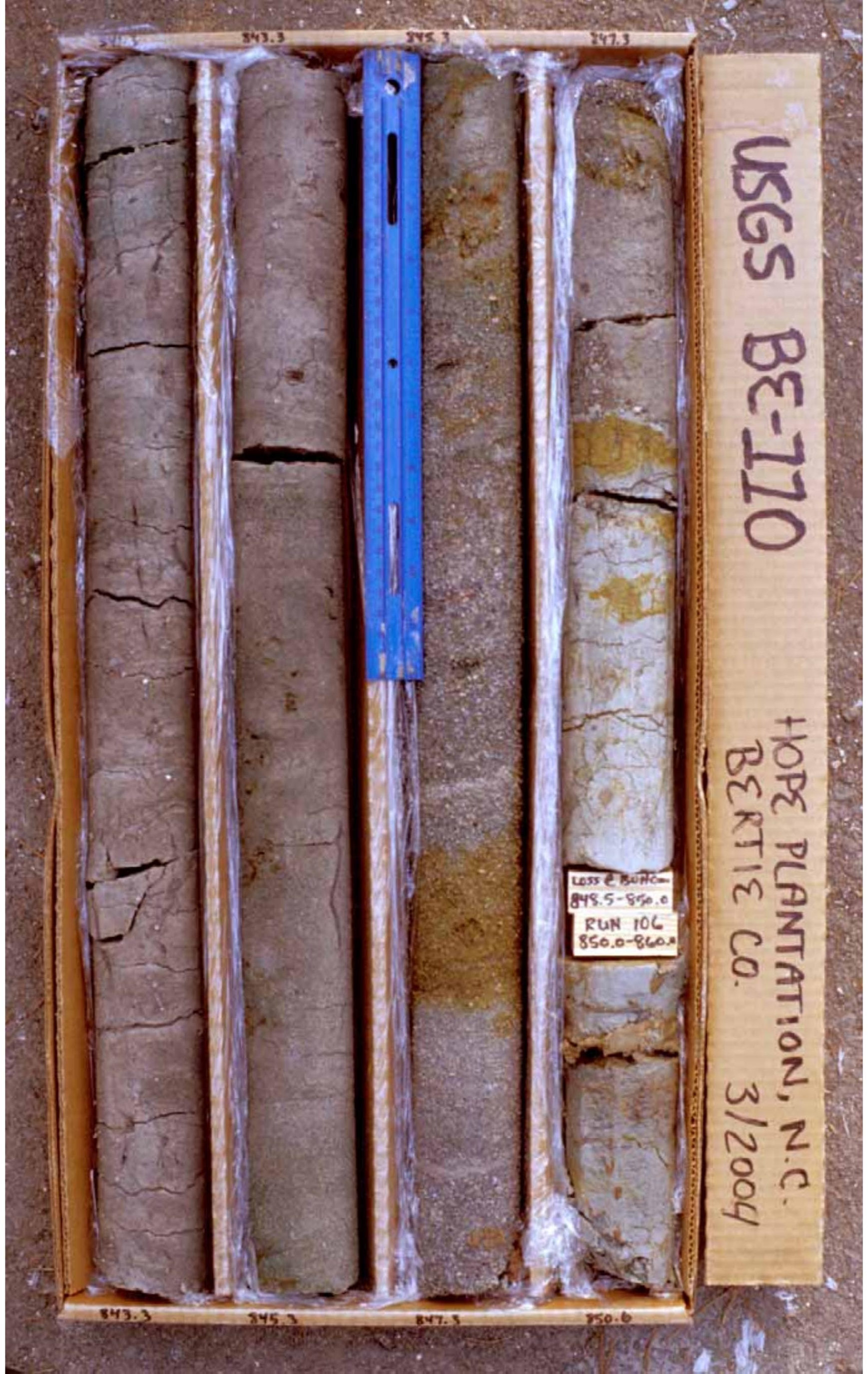




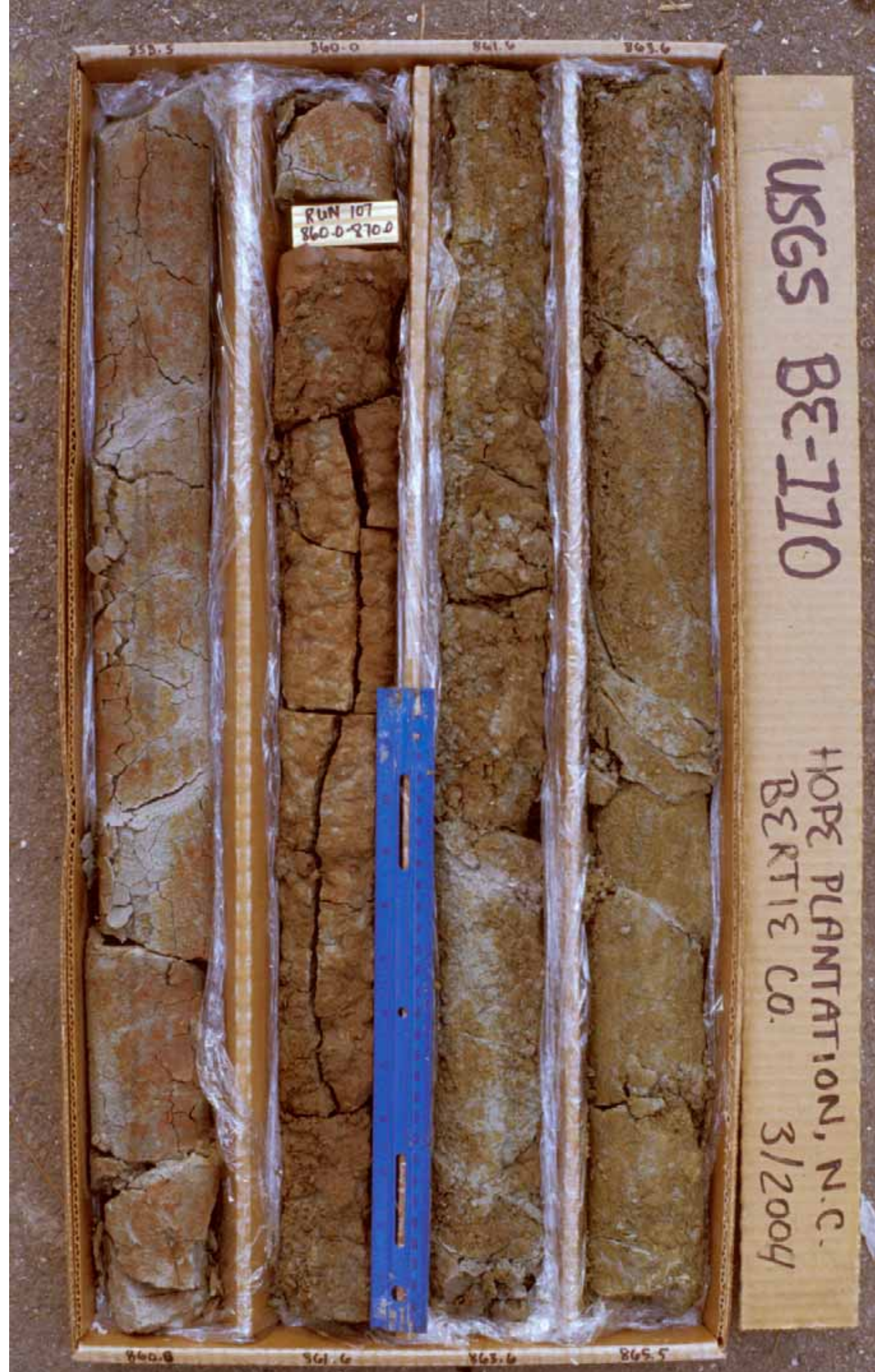




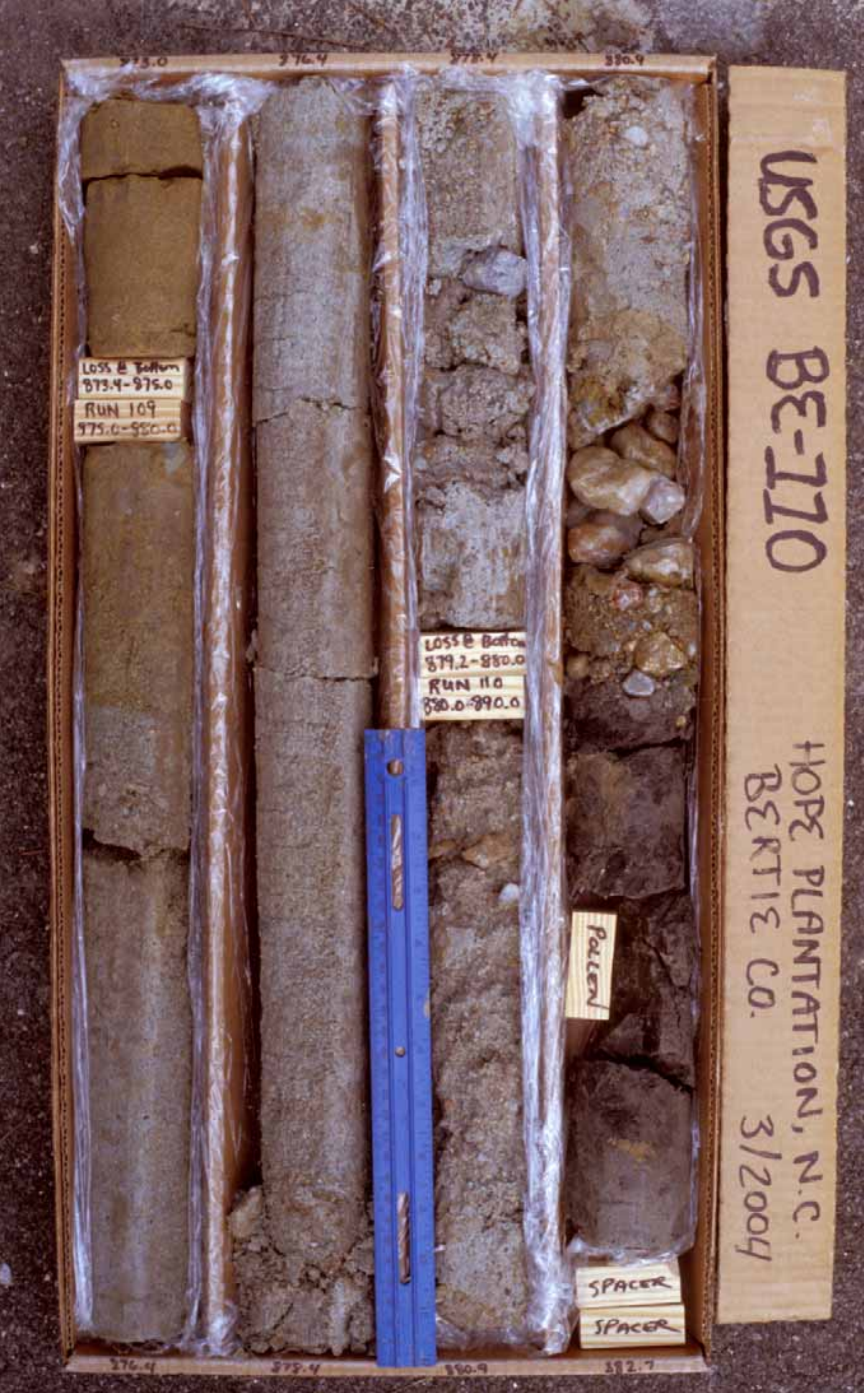




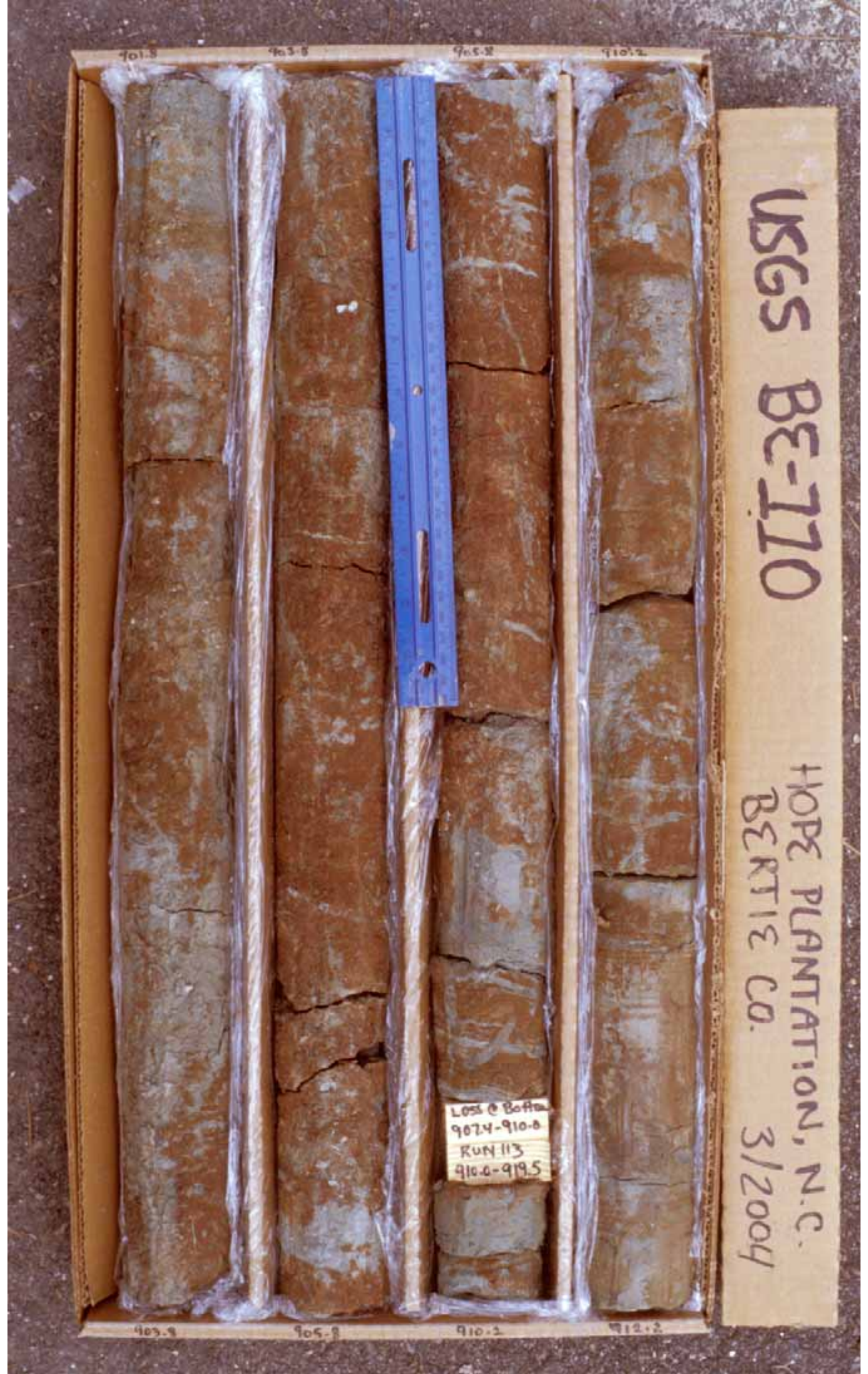




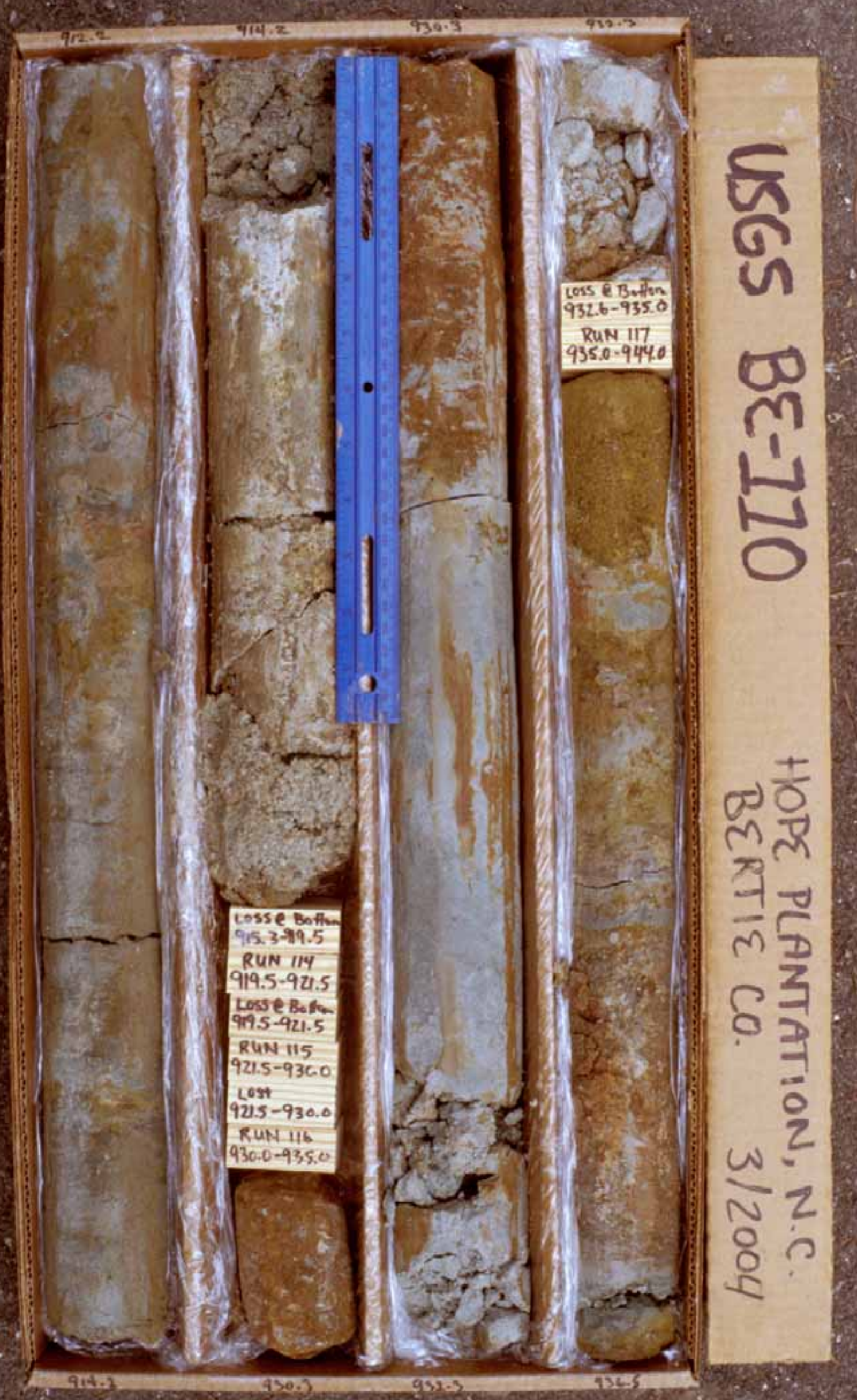




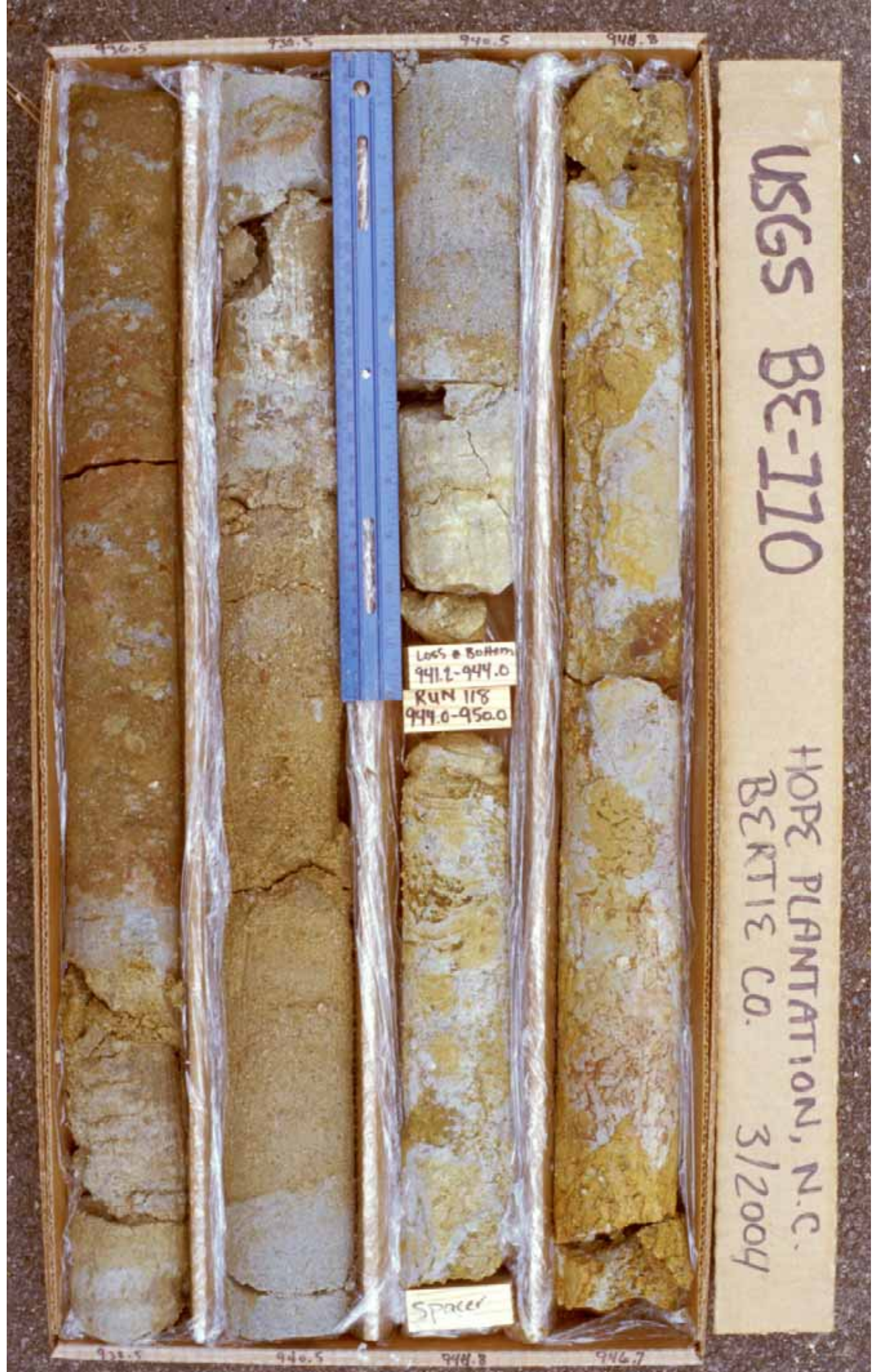




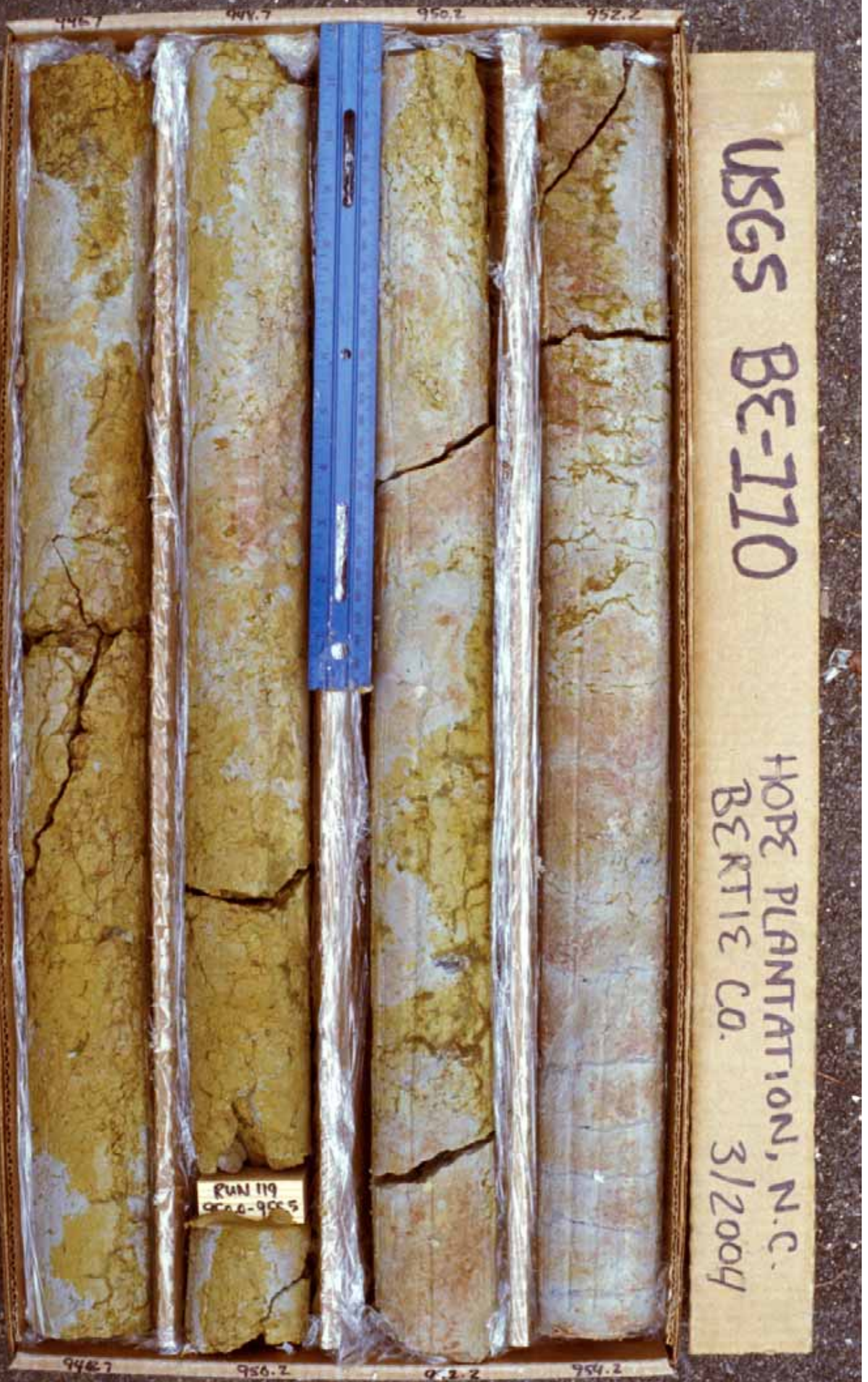




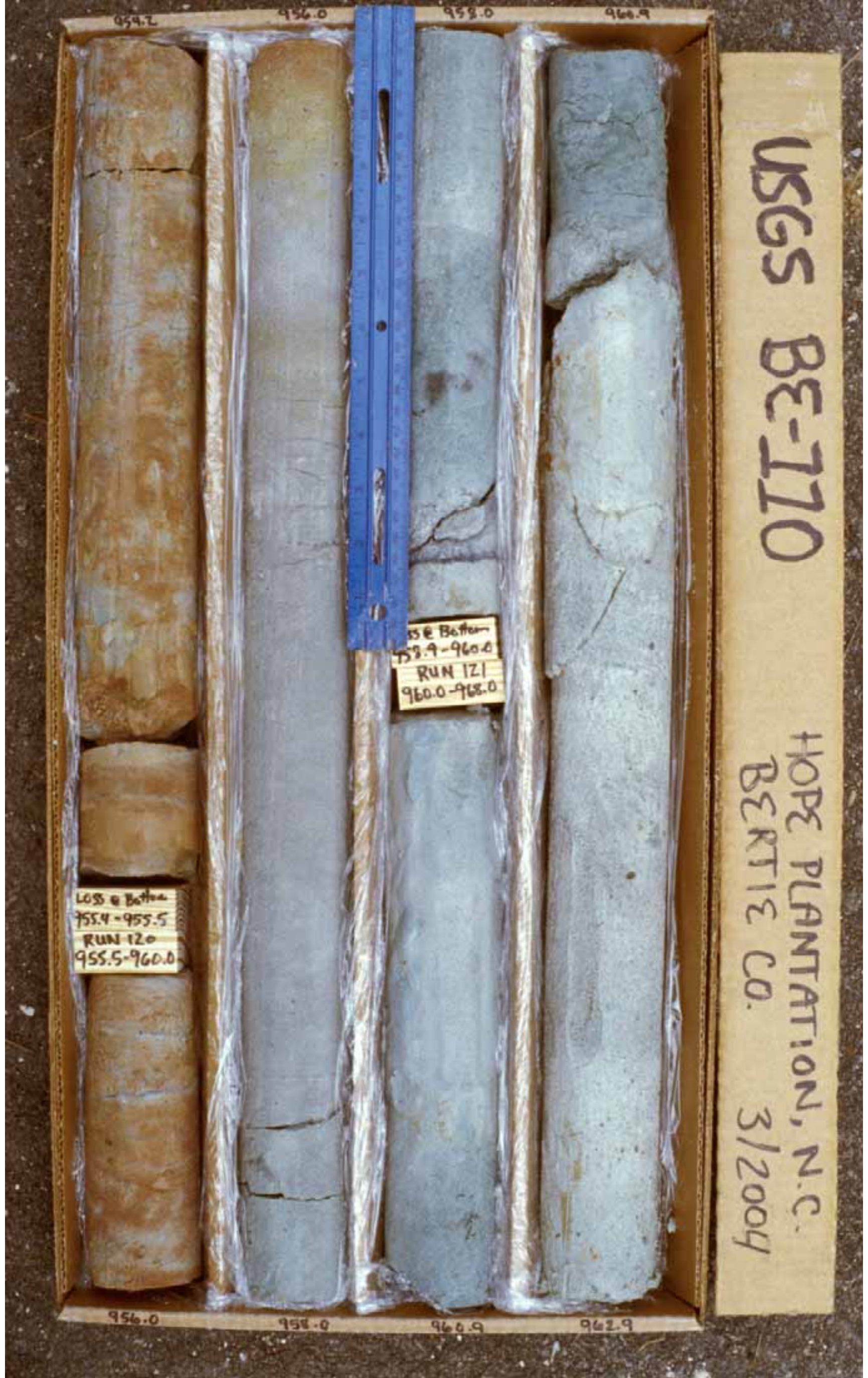




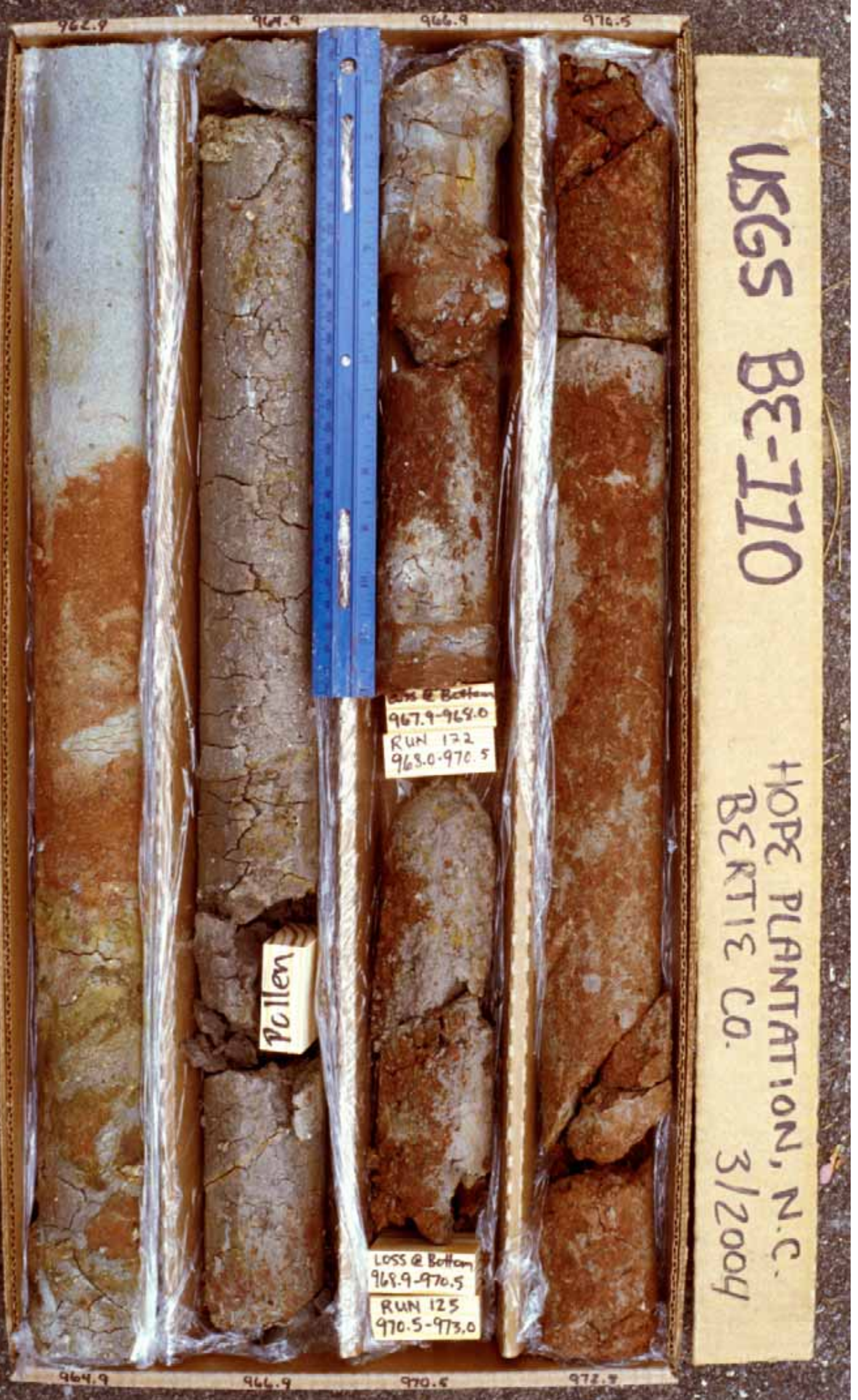




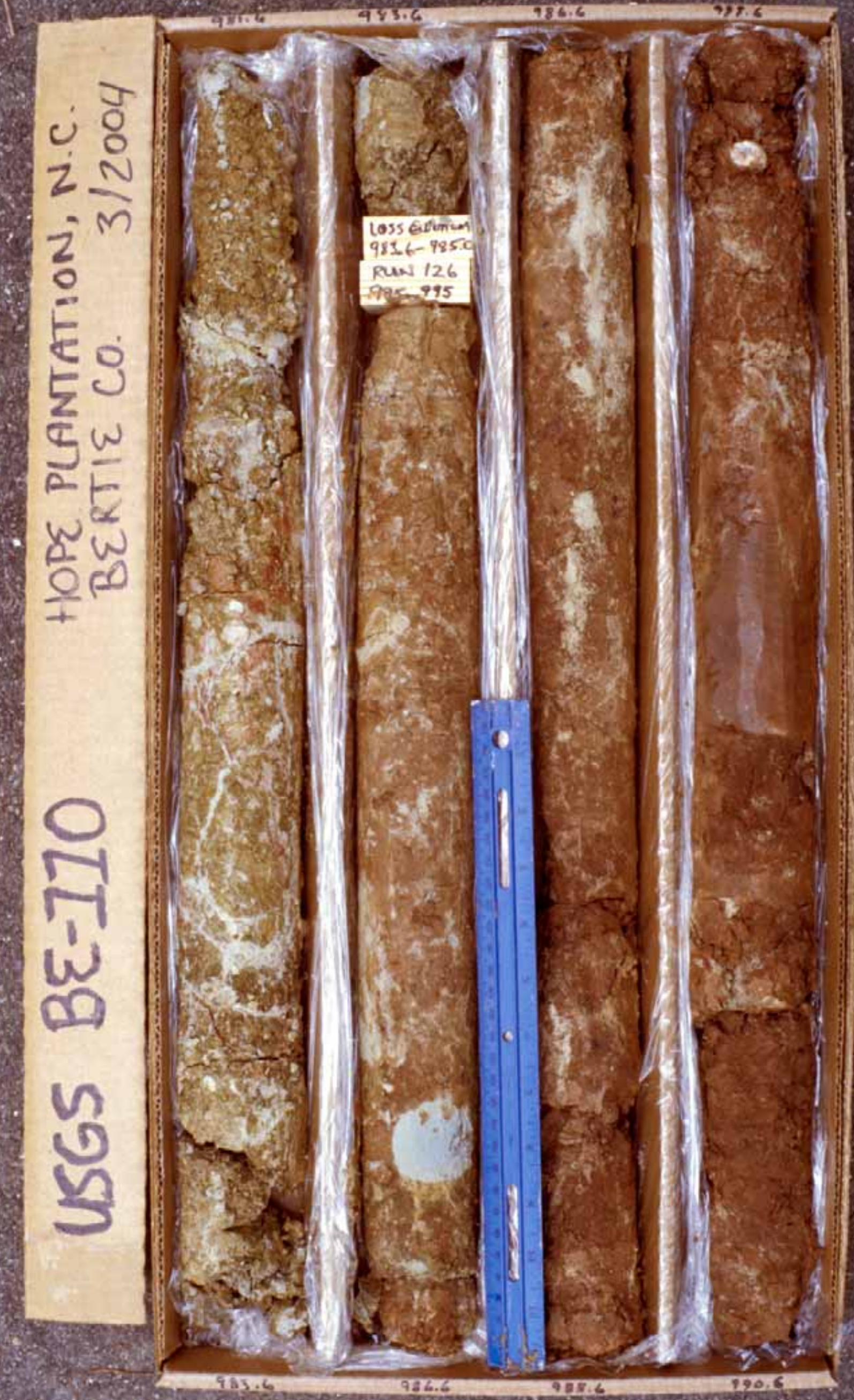




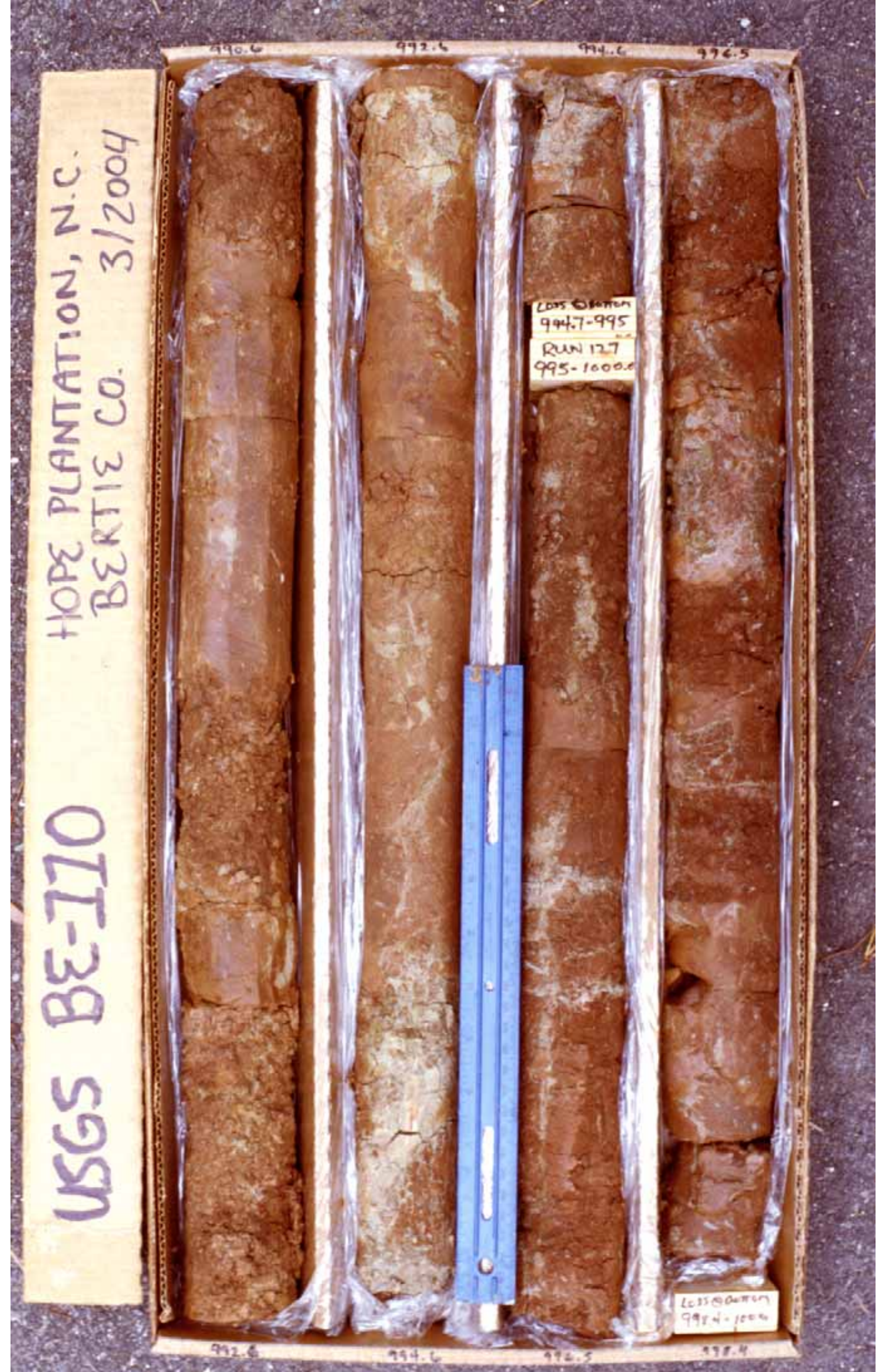




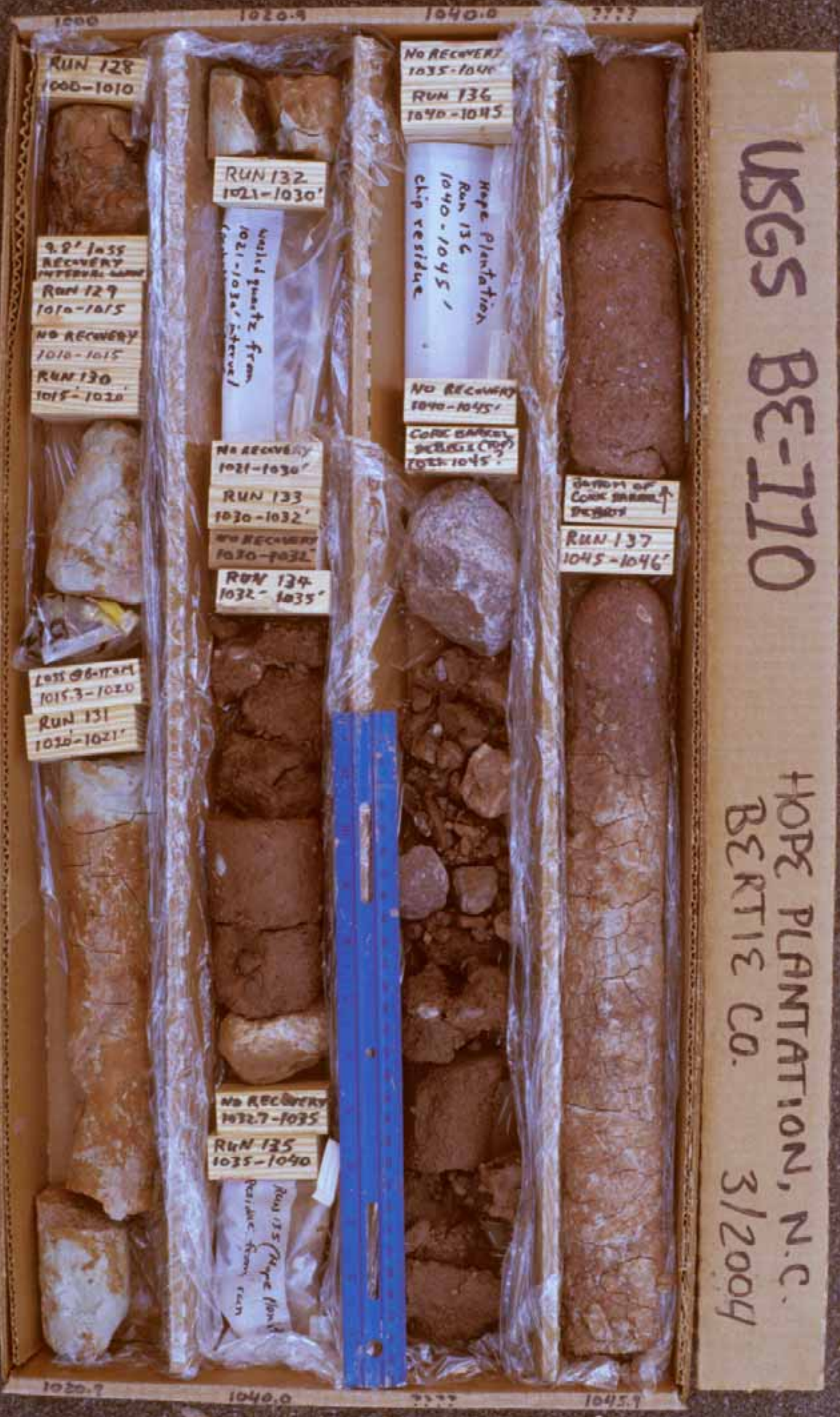




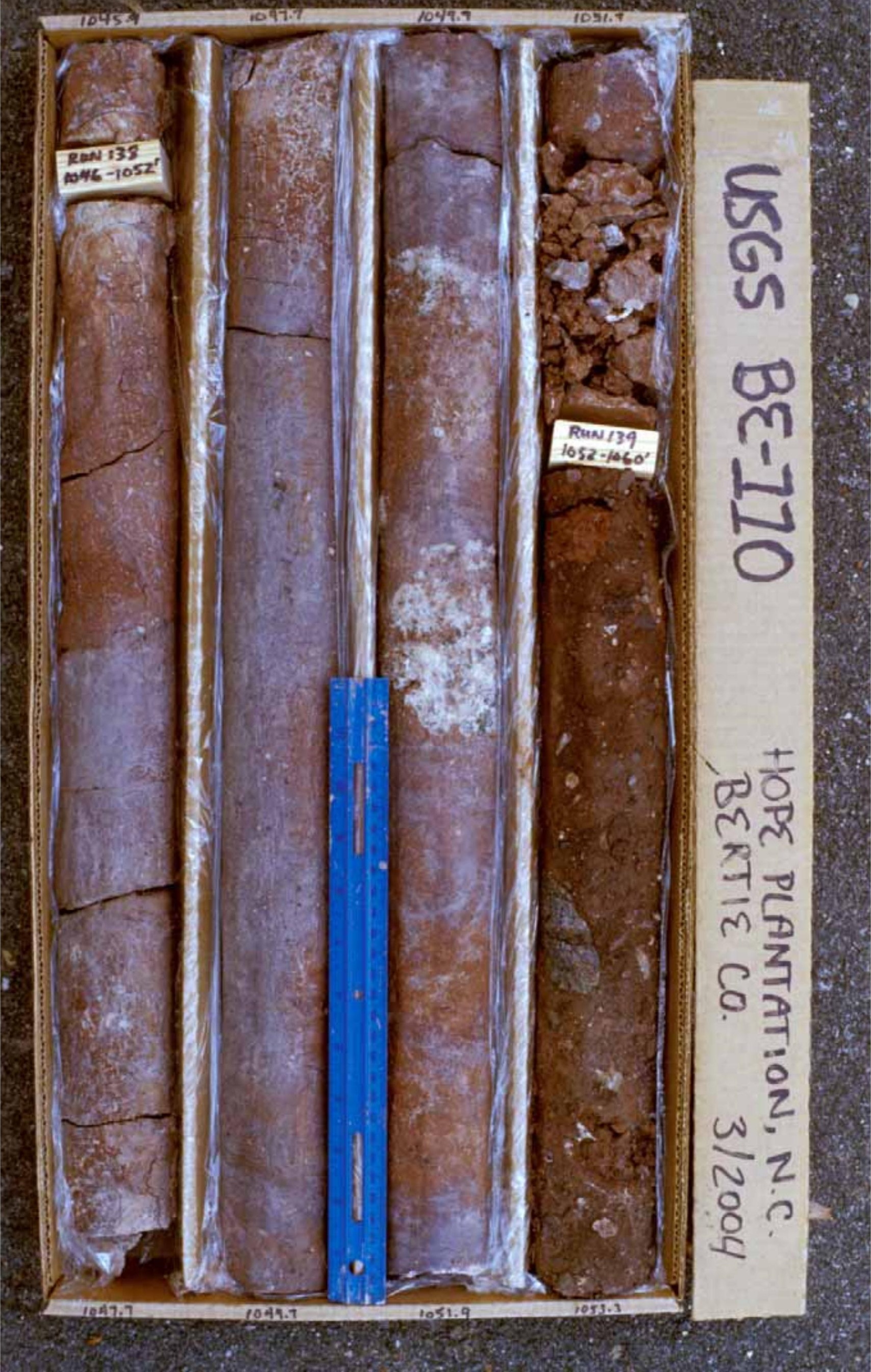




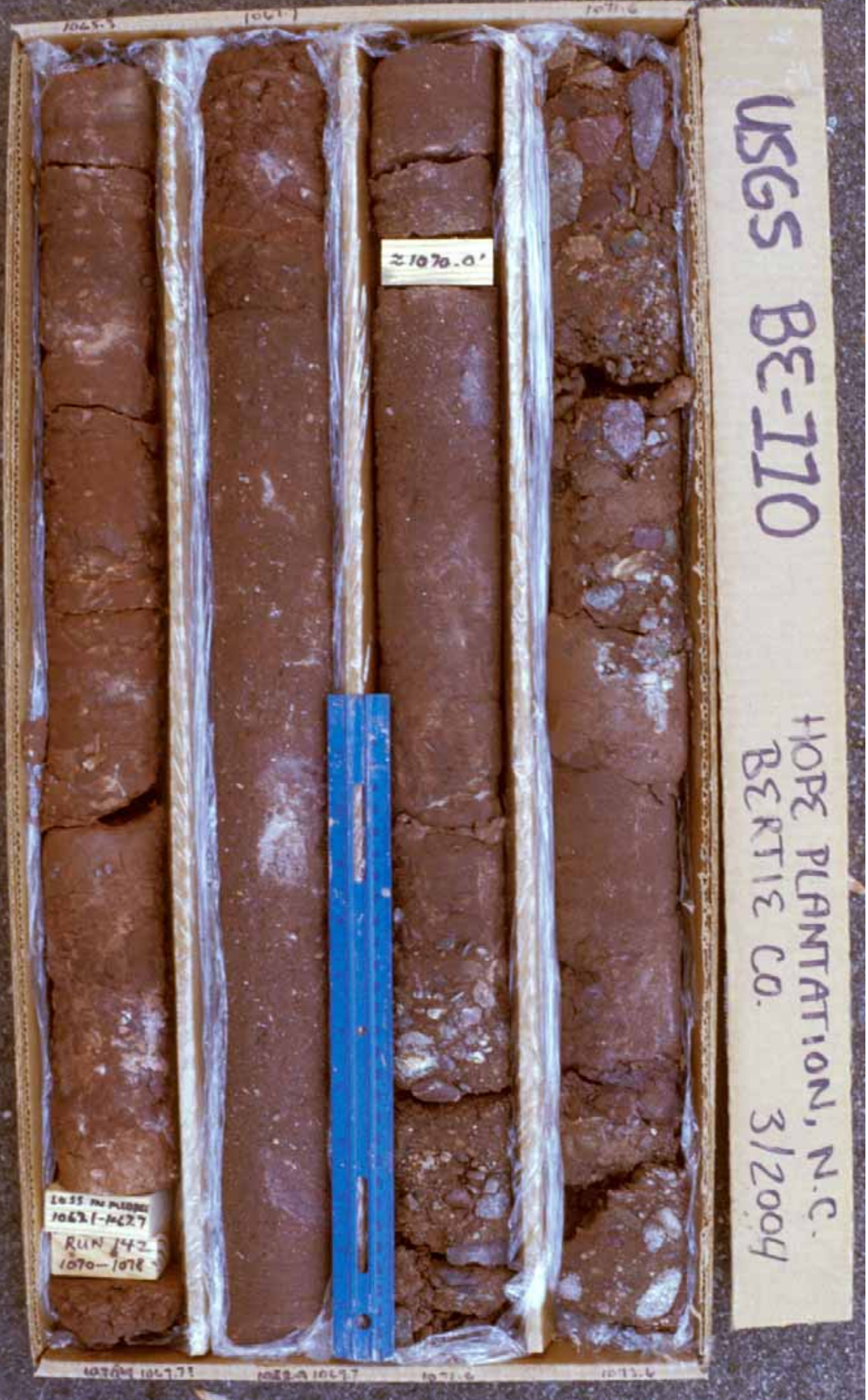




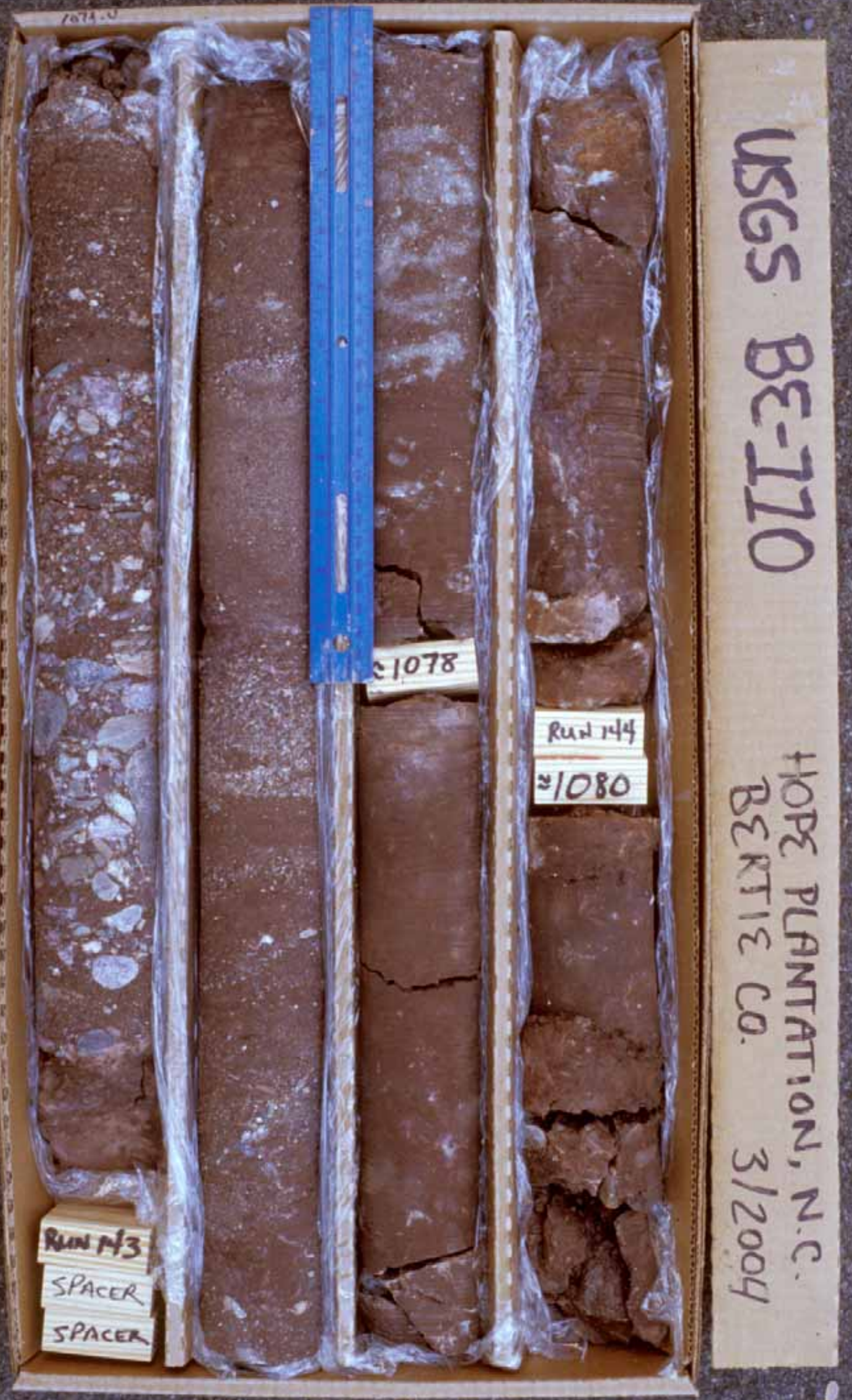





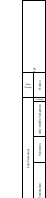

